\title{
Fluctuation-Dissipation: Response Theory in Statistical Physics
}

\author{
Umberto Marini Bettolo Marconi ${ }^{\mathrm{a}}$, Andrea Puglisi ${ }^{\mathrm{b}}$, \\ Lamberto Rondoni ${ }^{\mathrm{c}}$ and Angelo Vulpiani ${ }^{\mathrm{d}}$ \\ ${ }^{a}$ Università di Camerino, Dipartimento di Matematica e Fisica, Via Madonna \\ delle Carceri, I-62032 Camerino, Italy \\ b Università di Roma "La Sapienza", Dipartimento di Fisica, p.le Aldo Moro 2, \\ I-00185 Roma, Italy \\ ${ }^{\mathrm{c}}$ Dipartimento di Matematica and INFM, Politecnico di Torino, Corso Duca degli \\ Abruzzi 24, I-10129, Torino, Italy \\ "Università di Roma "La Sapienza", Dipartimento di Fisica and INFN, p.le Aldo \\ Moro 2, I-00185 Roma, Italy
}

\begin{abstract}
General aspects of the Fluctuation-Dissipation Relation (FDR), and Response Theory are considered. After analyzing the conceptual and historical relevance of fluctuations in statistical mechanics, we illustrate the relation between the relaxation of spontaneous fluctuations, and the response to an external perturbation. These studies date back to Einstein's work on Brownian Motion, were continued by Nyquist and Onsager and culminated in Kubo's linear response theory.

The FDR has been originally developed in the framework of statistical mechanics of Hamiltonian systems, nevertheless a generalized FDR holds under rather general hypotheses, regardless of the Hamiltonian, or equilibrium nature of the system. In the last decade, this subject was revived by the works on Fluctuation Relations (FR) concerning far from equilibrium systems. The connection of these works with large deviation theory is analyzed.

Some examples, beyond the standard applications of statistical mechanics, where fluctuations play a major role are discussed: fluids, granular media, nano-systems and biological systems.
\end{abstract}

Key words: Correlation functions, Non-equilibrium phenomena, Large deviations, Fluctuation Relations

PACS: 05.40.-a, 05.70.Ln, 45.70.-n, 47.52.+j, 02.50.Ga, 05.60.-k, 47.27.-i, 47.27.eb, $51.20 .+\mathrm{d}$ 


\section{Contents}

1 Introduction 4

1.1 Introductory remarks and plan of the paper

1.2 Historical overview 6

1.3 Fluctuations in equilibrium statistical mechanics 9

1.4 Fluctuations in non-equilibrium statistical mechanics: the Einstein relation between diffusion and mobility 11

2 The classical linear response theory 12

2.1 Hamiltonian formulation of the FDR 12

2.2 Green-Kubo relations 15

2.3 Linear Response for stochastic dynamics 17

2.4 Johnson-Nyquist spectrum and Onsager regression hypothesis 23

$3 \quad$ Fluctuation dissipation in chaotic systems and statistical physics 28

3.1 Chaos and the FDR: van Kampen's objection 28

3.2 Generalized FDR for non Hamiltonian systems 30

3.3 FDR, chaos and molecular dynamics 33

3.4 Linear response theory, foundations of statistical mechanics and anomalous diffusion 35

4 Some applications 40

4.1 Fluid dynamics 40

4.2 Beyond the linear FDR and climate 50

4.3 Granular materials 55

4.4 Further applications

$5 \quad$ Fluctuation relations 74

5.1 Irreversible entropy production $\quad 76$

5.2 Ergodic hypothesis: the Khinchin's approach 77 
5.3 Molecular dynamics models

5.4 Deterministic systems: transient and steady state relations 82

$6 \quad$ Fluctuation relations in stochastic systems and applications 97

6.1 Stochastic systems: bounded and unbounded state spaces 97

6.2 Numerical works 107

6.3 Experimental works 113

$\begin{array}{lll}7 & \text { Large deviations and FR } & 119\end{array}$

7.1 Onsager-Machlup approach to fluctuations of thermodynamic variables $\quad 119$

7.2 Large deviations in extended nonequilibrium systems 123

7.3 The additivity principle 131

8 Conclusions 133

A Appendix: Models of granular gases 134

B Appendix: Large Deviations in a nutshell 137

C Appendix: Anosov systems 139

D Appendix: A pedagogical example 143

References 
To the memory of Robert H. Kraichnan (1928-2008), whose conspicuous and remarkable achievements include fundamental contributions to the subject of our review.

\section{Introduction}

\subsection{Introductory remarks and plan of the paper}

Recent developments in non equilibrium statistical physics have convinced us that times are ripe for a review of the vast subject concerning the fluctuations of systems described by statistical mechanics. This issue is important even beyond the "traditional" applications of statistical mechanics, e.g. in a wide range of disciplines ranging from the study of small biological systems to turbulence, from climate studies to granular media etc. Moreover, the improved resolution in real experiments and the computational capability reached in numerical simulations has led to an increased ability to unveil the detailed nature of fluctuations, posing new questions and challenges to the theorists.

One of the most important and general results concerning systems described by statistical mechanics is the existence of a relation between the spontaneous fluctuations and the response to external fields of physical observables. This result has applications both in equilibrium statistical mechanics, where it is used to relate the correlation functions to macroscopically measurable quantities such as specific heats, susceptibilities and compressibilities, and in non equilibrium systems, where it offers the possibility of studying the response to time dependent external fields, by analyzing time-dependent correlations.

The idea of relating the amplitude of the dissipation to that of the fluctuations dates back to Einstein's work on Brownian motion. Later, Onsager put forward his regression hypothesis stating that the relaxation of a macroscopic nonequilibrium perturbation follows the same laws which govern the dynamics of fluctuations in equilibrium systems. This principle is the basis of the FDR theorem of Callen and Welton, and of Kubo's theory of time dependent correlation functions. This result represents a fundamental tool in non-equilibrium statistical mechanics since it allows one to predict the average response to external perturbations, without applying any perturbation. In fact, via an equilibrium molecular dynamics simulation one can compute correlation functions at equilibrium and then, using the Green-Kubo formula, obtain the transport coefficients of model liquids without resorting to approximation schemes. 
Although the FDR theory was originally applied to Hamiltonian systems near thermodynamic equilibrium, it has been realized that a generalized FDR holds for a vast class of systems with chaotic dynamics of special interest in the study of natural systems, such as geophysics and climate.

A renewed interest toward the theory of fluctuations has been motivated by the study of nonequilibrium processes. In 1993 Evans, Cohen and Morriss considered the fluctuations of the entropy production rate in a shearing fluid, and proposed the so called Fluctuation Relation (FR). This represents a general result concerning systems arbitrarily far from equilibrium. Moreover it is consistent with the Green-Kubo and Onsager relations, when equilibrium is approached. Starting with the developments of the FR proposed by Evans and Searles and, in different conditions, by Gallavotti and Cohen, continuing with the Crooks and Jarzynski relations, and the recent works by Derrida and coworkers and Jona-Lasinio and coworkers on dynamical path probabilities, the last 10-15 years have produced a whole new theoretical framework which encompasses the previous linear response theory and goes beyond that, to include far from equilibrium phenomena, such as turbulence and the dynamics of granular materials.

In this paper, we only consider systems which are ergodic and have an invariant phase space distribution, which is reached in physically relevant time scales. Therefore, we do not discuss aging and glassy behaviours. For such interesting issues the reader is referred to recent reviews $[1,2,3]$.

This review is organized as follows. In this section we give a brief historical overview on the origin of FDR, in particular of its relevance to the "proof" of the existence of atoms. In Section 2 we present the classical fundamental contributions of Onsager and Kubo to the general theory of FDR. The third Section is devoted to a discussion of FDR in chaotic systems. It is shown that a generalized FDR holds under rather general conditions, even in non-Hamiltonian systems, and in non-equilibrium situations. In addition, we discuss the connection between this FDR and the foundations of statistical mechanics, and anomalous diffusion. Section 4 treats non-standard applications of the FDR: fluid dynamics, climate, granular materials and biophysical systems. The different approaches to the FR are discussed in Section 5. In Section 6 we discuss the numerical and experimental investigations of the FR in stochastic systems, granular gases and conducting systems. Section 7 concerns the seminal work of Onsager and Machlup, and the recent results in extended non-equilibrium systems, in terms of large deviations theory. Four Appendices conclude the paper. 


\subsection{Historical overview}

The study of the fluctuations, and of their relation with the response to external perturbations, has an important conceptual and historical relevance, apart from its technical interest which is now universally recognized. Therefore we give a brief overview of Statistical Mechanics at the beginning of the 20-th century.

\subsubsection{Fluctuation Phenomena and Atoms}

In spite of the great successes of the atomistic hypothesis in chemistry and kinetic theory, towards the end of the 19-th century, some influential scientists such as Ostwald and Mach still claimed that it was possible to avoid the atomistic perspective in the descriptions of nature. Indeed, there was no unquestionable evidence of the existence of atoms at that time. The atomic theory plays a role in physics similar to that of certain auxiliary concepts in mathematics; it is a mathematical model for facilitating the mental reproduction of facts [4], was stated by Mach.

Even Boltzmann and Gibbs, who already knew the expression for the mean square energy fluctuation, correctly thought that fluctuations would be too small to be observed in macroscopic systems: In the molecular theory we assume that the laws of the phenomena found in nature do not essentially deviate from the limits that they would approach in the case of an infinite number of infinitely small molecules [5].

... [the fluctuations] would be in general vanishing quantities, since such experience would not be wide enough to embrace the more considerable divergences from the mean values [6].

One of the reasons of Einstein's and Smoluchowski's interest for statistical mechanics was to find conclusive evidence for the atomic hypothesis. At variance with the ideas of Boltzmann and Gibbs, their efforts attributed a central role to the fluctuations. The equation we finally obtained (eq. 1.11) would yield an exact determination of the universal constant (i.e. the Avogadro number), if it were possible to determine the average of the square of the energy fluctuation of the system; this is however not possible according our present knowledge [7].

\subsubsection{Brownian motion: a macroscopic window on the microscopic world}

Einstein's hope came true in the understanding of a "strange" phenomenon: the Brownian motion (BM). In 1827 the Scottish botanist Robert Brown no- 
ticed that pollen grains suspended in water jiggled about under the lens of the microscope, following a zigzag path. Initially, he believed that such an activity was peculiar to the male sexual cells of plants; further study revealed that the same motion could be observed even with chips of glass or granite or particles of smoke. For some decades such a phenomenon was considered as a sort of curiosity, although the BM attracted the interest of important scientists, such as Poincaré [8].

The breakthrough in the understanding of the BM is due to the independent works by Einstein [9,10] and Smoluchowski [11] at the beginning of the 20th century. A few years later, Langevin proposed an approach in terms of a stochastic differential equation, which takes into account the effect of molecular collisions by means of an average force, given by the fluid friction, and of a random fluctuating term [12].

The experimental works by Perrin and Svendborg on the BM can be considered as the first "proof" of the existence of atoms, while Langevin's equation, which has been the first example of stochastic differential equation, inspired the mathematical theory of continuous time stochastic processes.

The basic physical assumptions in both Einstein's and Langevin's approaches are:

a) Stokes' law for the frictional force exerted on a body moving in a liquid;

b) the equipartition of kinetic energy among the degrees of freedom of the system, i.e. between the particles of the fluid and the grain performing BM.

A colloidal particle suspended in a liquid at temperature $T$ is thus assimilated to a particle of the liquid, so that it possesses an average kinetic energy $R T /\left(2 N_{A}\right)$, in each spatial direction, where $R$ is the perfect gas constant and $N_{A}$ is the Avogadro number. Accordingly, one obtains:

$$
\frac{1}{2} m\left\langle v_{x}^{2}\right\rangle=\frac{R T}{2 N_{A}}
$$

According to Stokes' law, a spherical particle of radius $a$, moving in a liquid with speed $V$ in the $x$ direction, experiences a viscous drag force:

$$
f_{S}=-\alpha V=-6 \pi \eta a V
$$

where $\eta$ is the viscosity. This law holds if $a$ is much larger than the average distance between the liquid molecules, in which case Stokes' force $\left(f_{S}\right)$ represents the average macroscopic effect of the irregular impacts of the molecules of the fluid. 
Therefore, isolating the average force, the dynamical equation of the particle in the $x$ direction can be written as

$$
m \frac{d V}{d t}=f_{S}+f_{R}(t)=-\alpha V+f_{R}(t)
$$

where $f_{R}(t)$ is a random fluctuating force mimicking the effects of the impact of the molecules on the particle. Of course $\left\langle f_{R}(t)\right\rangle=0$, and its characteristic time, being determined by the collisions of the molecules of the liquid with the colloidal particle, is much smaller than $\tau=m / \alpha$. One can then assume that $f_{R}$ is a Gaussian stochastic process with $\left\langle f_{R}(t) f_{R}\left(t^{\prime}\right)\right\rangle=c \delta\left(t-t^{\prime}\right)$, where $c$ can be determined by the energy equipartition. Elementary computations give [13]

$$
\left\langle[x(t)-x(0)]^{2}\right\rangle=\frac{2 R T}{\alpha N_{A}}\left[t-\frac{m}{\alpha}\left(1-e^{-\frac{\alpha}{m} t}\right)\right] .
$$

For a grain of size $O(1 \mu)$, in a common liquid (such as water) at room temperature, the characteristic time $\tau=m / \alpha=m / 6 \pi \eta a$ is $O\left(10^{-7} s\right)$. For $t \gg \tau$, we get

$$
\left\langle[x(t)-x(0)]^{2}\right\rangle \simeq \frac{2 R T}{\alpha N_{A}} t=\frac{R T}{3 N_{A} \pi \eta a} t .
$$

This leads to the celebrated Einstein relation for the diffusion coefficient $D$, in terms of macroscopic variables and of the Avogadro number:

$$
D=\lim _{t \rightarrow \infty} \frac{\left\langle[x(t)-x(0)]^{2}\right\rangle}{2 t}=\frac{R T}{6 N_{A} \pi \eta a} .
$$

Let us stress that (1.5) allows the determination of the Avogadro number (a microscopic quantity) from experimentally accessible macroscopic quantities, thus providing a non ambiguous link between the microscopic and macroscopic levels of description, in physics.

The theoretical work by Einstein and the experiments by Perrin gave a clear and conclusive evidence of the relationship between the diffusion coefficient (which is measurable at the macroscopic level) and the Avogadro number (which is related to the microscopic description) [14]. Such a result could be considered as a "proof" of the existence of atoms: after that, even two champions of the energetic point of view, like Helm and Ostwald, accepted atomism as a physical fact and not as a mere useful hypothesis. In a lecture in Paris, in 1911, Arrhenius, summarizing the works of Einstein and Perrin, declared after this, it does not seem possible to doubt that the molecular theory entertained by the philosophers of antiquity, Leucippus and Democritos, has attained the truth at least in essentials [8].

Although Langevin's approach, like Einstein's, is, from a mathematical point of view, rather simple, there is a very subtle conceptual point at the basis of his theory of the Brownian motion. The ingenious idea is the assumption of 
the validity of Stokes law (which has a macroscopic nature), together with the assumption that the Brownian particle is in statistical equilibrium with the molecules in the liquid. In other words, in spite of the fact that the mass of the colloidal particle is exceedingly larger than the mass of the molecules, energy equipartition is assumed to hold.

\subsection{Fluctuations in equilibrium statistical mechanics}

Equilibrium statistical mechanics predicts both the average value of the thermodynamic observables and the size of their fluctuations about their equilibrium values. In this section, we briefly report the salient features of the theory of fluctuations of extensive thermodynamic variables.

Let us consider a large isolated system in thermodynamic equilibrium, and single out a small region representing the subsystem of interest (here named $\mathrm{S}$ ), while the rest of the system constitutes a reservoir (R). Suppose that, due to the coupling with $\mathrm{R}$, the extensive variable $X$ of the system $\mathrm{S}$ can fluctuate about its equilibrium value, while the remaining extensive variables are held fixed. The probability of observing a fluctuation of size smaller than a value $X_{0}$ is given by the canonical distribution function:

$$
P\left(X<X_{0}\right)=e^{-\psi(h)} \int_{-\infty}^{X_{0}} e^{-h X} d N(X)
$$

where $h$ is the intensive thermodynamic field conjugated to $X, N(X)$ is the cumulative number of microstates with $X<X_{0}$, and the integral is to be interpreted in the Riemann-Stieltjes sense if the spectrum has also a discrete part. The function $\psi(h)$, which normalizes the distribution function, is determined by the condition

$$
e^{\psi(h)}=\int_{-\infty}^{\infty} e^{-h X} d N(X)
$$

and represents a thermodynamic potential. The function $\psi(h)$ is a Legendre transform of the entropy, or generalized Massieu function [15]. Note that, even if this argument is formal and therefore of general validity, when $X$ is an extensive observable of a macroscopic system (i.e. in the thermodynamic limit) the function $\psi(h)$, or better its Legendre transform, can be seen in terms of large deviations theory, see Appendix B.

If we consider the characteristic function:

$$
\left\langle e^{\alpha X}\right\rangle=e^{-\psi(h)} \int_{-\infty}^{\infty} e^{-(h-\alpha) X} d N(X)=e^{\psi(h-\alpha)} e^{-\psi(h)}
$$

and expand $\psi(h-\alpha)$ with respect to $\alpha$, we obtain the cumulants, $\left\langle X^{n}\right\rangle_{c}$, of 
the distribution

$$
\ln \left\langle e^{\alpha X}\right\rangle=\sum_{n=1}^{\infty} \frac{\alpha^{n}}{n !}\left\langle X^{n}\right\rangle_{c}=\sum_{n=1}^{\infty} \frac{(-\alpha)^{n}}{n !} \frac{d^{n}}{d h^{n}} \psi(h)
$$

As an important example, we consider the energy fluctuations in a system $\mathrm{S}$, at constant temperature. In this case $X=E, h=\beta^{-1}$ and $\psi(h)=-\beta A(\beta)$, where $A$ is the Helmholtz free energy. By employing eq. (1.9) and using standard thermodynamic relations, we obtain the following results:

$$
\begin{aligned}
\langle E\rangle_{c} & \equiv\langle E\rangle=-\frac{d}{d \beta}(\beta A(\beta))=U \\
\left\langle E^{2}\right\rangle_{c} & \equiv\left\langle E^{2}\right\rangle-\langle E\rangle^{2}=\frac{d^{2}(\beta A(\beta))}{d \beta^{2}}=-\frac{d U}{d \beta}=k_{B} T^{2} C_{V} \\
\left\langle E^{3}\right\rangle_{c} & \equiv\left\langle E^{3}\right\rangle-3\langle E\rangle\left\langle E^{2}\right\rangle+2\langle E\rangle^{3}=-\frac{d^{3}(\beta A(\beta))}{d \beta^{3}} \\
& =k_{B}^{2} T^{3}\left[2 C_{V}+T \frac{d C_{V}}{d T}\right] .
\end{aligned}
$$

Formulae (1.10)-(1.13) are a manifestation of the relation between the fluctuations of the quantity $X$ and the variations of the thermodynamic potential $\psi$, with respect to the conjugate field $h$. The generalization to several extensive variables $X_{1}, X_{2} . ., X_{r}$ is straightforward and involves the generalized potential defined by $\phi\left(h_{1}, h_{2}, . ., h_{r}\right)$

$$
\begin{gathered}
e^{\phi\left(h_{1}, h_{2}, . ., h_{r}\right)}=\int . . \int d X_{1} . . d X_{r} e^{\left(h_{1} X_{1}+. .+h_{r} X_{r}\right)} d N\left(X_{1}, . ., X_{r}\right) \\
\left\langle\exp \sum_{j}^{r} \alpha_{j} X_{j}\right\rangle=\mathrm{e}^{-\phi\left(h_{1}, h_{2}, . ., h_{r}\right)} \int . . \int d X_{1} . . d X_{r} \exp \left(\sum_{j}^{r}\left(\alpha_{j}-h_{j}\right) X_{j}\right) d N\left(X_{1}, ., X_{r}\right) \\
\left\langle X_{m}\right\rangle_{c}=-\frac{\partial}{\partial h_{m}} \psi\left(h_{1}, . ., h_{r}\right) \\
\left\langle X_{m} X_{n}\right\rangle_{c}=\frac{\partial^{2}}{\partial h_{m} \partial h_{n}} \psi\left(h_{1}, . ., h_{r}\right) .
\end{gathered}
$$

Let us, now, consider the problem of relating the properties of a system whose Hamiltonian is $\mathcal{H}(\mathbf{P}, \mathbf{Q})=\mathcal{H}_{0}(\mathbf{P}, \mathbf{Q})+\lambda A(\mathbf{P}, \mathbf{Q})$ to those of a similar system characterized by $\mathcal{H}_{0}(\mathbf{P}, \mathbf{Q})$ only, where $\mathbf{P}=\left(\mathbf{p}_{1}, \ldots, \mathbf{p}_{N}\right), \mathbf{Q}=\left(\mathbf{q}_{1}, \ldots, \mathbf{q}_{N}\right)$. If the perturbation induced by $\lambda A(\mathbf{P}, \mathbf{Q})$ is small, the canonical distribution of 
the system with $\lambda \neq 0$ is to linear order given by:

$$
f(\mathbf{P}, \mathbf{Q})=\frac{\exp (-\beta \mathcal{H})}{\int d \mathbf{P} d \mathbf{Q} \exp (-\beta \mathcal{H})} \simeq \frac{\exp \left(-\beta \mathcal{H}_{0}\right)}{\int d \mathbf{P} d \mathbf{Q} \exp \left(-\beta \mathcal{H}_{0}\right)} \frac{1-\lambda \beta A(\mathbf{P}, \mathbf{Q})}{1-\lambda \beta\langle A(\mathbf{P}, \mathbf{Q})\rangle_{0}}
$$

or

$$
f(\mathbf{P}, \mathbf{Q})=f_{0}(\mathbf{P}, \mathbf{Q})\left(1-\lambda \beta\left[A(\mathbf{P}, \mathbf{Q})-\langle A(\mathbf{P}, \mathbf{Q})\rangle_{0}\right]\right)
$$

where \langle\rangle$_{0}$ stands for the average relative to the system with $\lambda=0$. The corresponding average change of a generic observable $B(\mathbf{P}, \mathbf{Q})$, induced by the perturbation, is to the same order:

$$
\begin{aligned}
\langle\Delta B\rangle_{0} & \simeq \int d \mathbf{P} d \mathbf{Q} B(\mathbf{P}, \mathbf{Q})\left(f(\mathbf{P}, \mathbf{Q})-f_{0}(\mathbf{P}, \mathbf{Q})\right) \\
& \left.\left.\simeq-\lambda \beta \int d \mathbf{P} d \mathbf{Q} B(\mathbf{P}, \mathbf{Q})\left(A(\mathbf{P}, \mathbf{Q})-\langle A(\mathbf{P}, \mathbf{Q})\rangle_{0}\right)\right) f_{0}(\mathbf{P}, \mathbf{Q})\right) \\
& =-\lambda \beta\left(\langle B A\rangle_{0}-\langle B\rangle_{0}\langle A\rangle_{0}\right)
\end{aligned}
$$

The last formula shows that the change of the observable $B$ is related to the equilibrium pair correlation of $B$ with the perturbation $A$. With the choice $B(\mathbf{P}, \mathbf{Q})=A(\mathbf{P}, \mathbf{Q})=\mathcal{H}_{0}$ we obtain:

$$
\frac{\partial\left\langle\mathcal{H}_{0}\right\rangle_{0}}{\partial \beta}=\lim _{\lambda \rightarrow 0} \frac{\left\langle\Delta \mathcal{H}_{0}\right\rangle_{0}}{\lambda \beta}=-\left(\left\langle\mathcal{H}_{0}^{2}\right\rangle_{0}-\left\langle\mathcal{H}_{0}\right\rangle_{0}^{2}\right)=-k_{B} T^{2} C_{V}
$$

hence we connect the heat capacity, i.e. the response to an energy perturbation, to the energy fluctuations.

Another relevant instance of the equilibrium fluctuation-response theorem is the relation between the correlation function $\Gamma\left(r, r^{\prime}\right)$ (defined below) and the isothermal susceptibility $\chi_{T}\left(r, r^{\prime}\right)$. In this case one considers the Hamiltonian to be a functional of the magnetization density $m(r)$ :

$$
\mathcal{H}(m(r))=\mathcal{H}_{0}(m(r))-\int d r h(r) m(r)
$$

By using formula (1.20) with $B=m(r)$ and $\lambda A=\int d r^{\prime} h\left(r^{\prime}\right) m\left(r^{\prime}\right)$ we find:

$$
\chi_{T}\left(r, r^{\prime}\right)=\beta\left[\left\langle m(r) m\left(r^{\prime}\right)\right\rangle_{0}-\langle m(r)\rangle_{0}\left\langle m\left(r^{\prime}\right)\right\rangle_{0}\right]=\beta \Gamma\left(r, r^{\prime}\right) .
$$

1.4 Fluctuations in non-equilibrium statistical mechanics: the Einstein relation between diffusion and mobility

We already discussed the BM and its historical relevance for the atomic hypothesis and the development of the modern theory of stochastic processes. 
In addition, in the celebrated paper of Einstein one can find the first example of FDR. Let us write the Langevin equation for the colloidal particle in "modern" terms

$$
\frac{d V}{d t}=-\gamma V+\sqrt{\frac{2 \gamma k_{B} T}{m}} \eta
$$

where $\gamma=\alpha / m$ and $\eta$ is a white noise, i.e. a Gaussian stochastic process with $<\eta(t)>=0$ and $<\eta(t) \eta\left(t^{\prime}\right)>=\delta\left(t-t^{\prime}\right)$. The time correlation of $V$ is

$$
C_{V V}(t)=<V(t) V(0)>=<V^{2}>e^{-\gamma t},
$$

an easy computation gives

$$
D=\int_{0}^{\infty} C_{V V}(t) d t
$$

Consider now a small perturbing force $f(t)=F \Theta(t)$, where $\Theta(t)$ is the Heaviside step function. The average response of the velocity after a long time (i.e. the drift) to such a perturbation is given by:

$$
<\delta V>=\frac{F}{\gamma} \text {. }
$$

Defining the mobility $\mu$ as:

$$
<\delta V>=\mu F
$$

one easily obtains the celebrated Einstein relation

$$
\mu=\frac{D}{k_{B} T}
$$

which gives a link between the diffusion coefficient (a property of the unperturbed system) and the mobility, which measures the system's reaction to a small perturbation. This constitutes the first example of FDR.

\section{The classical linear response theory}

\subsection{Hamiltonian formulation of the FDR}

In this section we recall the derivation of Kubo's formula, which holds in the case of equilibrium dynamics [16]. Let us consider a system described by the Hamiltonian $\mathcal{H}(\mathbf{P}, \mathbf{Q}, t)=\mathcal{H}_{0}(\mathbf{P}, \mathbf{Q})-\mathcal{F}(t) A(\mathbf{P}, \mathbf{Q})$, where $\mathcal{H}_{0}(\mathbf{P}, \mathbf{Q})$ is the

time-independent part. The evolution is governed by the Hamilton equations: 


$$
\begin{aligned}
\frac{\partial q_{j}}{\partial t} & =\frac{\partial \mathcal{H}_{0}}{\partial p_{j}}-\mathcal{F}(t) \frac{\partial A}{\partial p_{j}}=\frac{\partial \mathcal{H}_{0}}{\partial p_{j}}-\mathcal{F}(t) K_{j}^{q} \\
\frac{\partial p_{j}}{\partial t} & =-\frac{\partial \mathcal{H}_{0}}{\partial q_{j}}+\mathcal{F}(t) \frac{\partial A}{\partial q_{j}}=-\frac{\partial \mathcal{H}_{0}}{\partial q_{j}}-\mathcal{F}(t) K_{j}^{p} .
\end{aligned}
$$

where we have introduced the "generalized forces"

$$
K_{j}^{q}=\frac{\partial A}{\partial p_{j}}, \quad K_{j}^{p}=-\frac{\partial A}{\partial q_{j}} .
$$

The associated $\Gamma$-phase space probability distribution evolves according to the Liouville equation:

$$
\frac{\partial}{\partial t} f(\mathbf{P}, \mathbf{Q}, t)+i\left[\mathcal{L}_{0}+\mathcal{L}_{e x t}(t)\right] f(\mathbf{P}, \mathbf{Q}, t)=0
$$

where $\mathcal{L}_{0}$ and $\mathcal{L}_{\text {ext }}$ are the Liouville operators relative to the unperturbed Hamiltonian and to its perturbation respectively. These can be expressed by means of Poisson brackets as:

$$
\begin{gathered}
i \mathcal{L}_{0} f=\left\{f, \mathcal{H}_{0}\right\}=\sum_{j}\left(\frac{\partial \mathcal{H}_{0}}{\partial p_{j}} \frac{\partial}{\partial q_{j}}-\frac{\partial \mathcal{H}_{0}}{\partial q_{j}} \frac{\partial}{\partial p_{j}}\right) f \\
i \mathcal{L}_{\text {ext }}(t) f=-\mathcal{F}(t)\{f, A\}=-\mathcal{F}(t) \sum_{j}\left(\frac{\partial A}{\partial p_{j}} \frac{\partial}{\partial q_{j}}-\frac{\partial A}{\partial q_{j}} \frac{\partial}{\partial p_{j}}\right) f .
\end{gathered}
$$

We assume that the system is in thermal equilibrium at $t=t_{0}$, when $\mathcal{H}=$ $\mathcal{H}_{0}$, and its statistical properties are described by the canonical equilibrium distribution, $f\left(\mathbf{P}, \mathbf{Q}, t_{0}\right)=f_{e q}(\mathbf{P}, \mathbf{Q})$, satisfying the equation $i \mathcal{L}_{0} f_{e q}(\mathbf{P}, \mathbf{Q})=$ 0 . Switching on the perturbation, we obtain an approximate solution of (2.4), in the form:

$$
f(\mathbf{P}, \mathbf{Q}, t)=f_{e q}(\mathbf{P}, \mathbf{Q})-i \int_{t_{0}}^{t} d t^{\prime} e^{-i\left(t-t^{\prime}\right) \mathcal{L}_{0}} \mathcal{L}_{e x t}\left(t^{\prime}\right) f_{e q}(\mathbf{P}, \mathbf{Q})+\ldots
$$

which is valid to first order in $i \mathcal{L}_{\text {ext }}(t)$. Using eq. (2.7), we can compute, to linear order in $\mathcal{F}(t)$, the ensemble average of an arbitrary function $B(\mathbf{P}, \mathbf{Q})$ of the phase space variables $(\mathbf{P}, \mathbf{Q})$, at time $t$, as follows:

$$
\langle B(t)\rangle=\int d \mathbf{P} d \mathbf{Q} B(\mathbf{P}, \mathbf{Q}) f(\mathbf{P}, \mathbf{Q}, t)
$$

so that its change with respect to the equilibrium value is:

$$
\begin{aligned}
& \langle\Delta B(t)\rangle=\langle B(t)\rangle-\left\langle B\left(t=t_{0}\right)\right\rangle= \\
& \quad-i \int d \mathbf{P} d \mathbf{Q} B(\mathbf{P}, \mathbf{Q}) \int_{t_{0}}^{t} d t^{\prime} e^{-i\left(t-t^{\prime}\right) \mathcal{L}_{0}} \mathcal{L}_{e x t}\left(t^{\prime}\right) f_{e q}(\mathbf{P}, \mathbf{Q})
\end{aligned}
$$


Substituting, we find

$$
\langle\Delta B(t)\rangle=\int d \mathbf{P} d \mathbf{Q} B(\mathbf{P}, \mathbf{Q}) \int_{t_{0}}^{t} d t^{\prime} e^{-i\left(t-t^{\prime}\right) \mathcal{L}_{0}} \mathcal{F}\left(t^{\prime}\right)\left\{f_{e q}, A\right\}
$$

Using (2.6) and the fact that $f_{\text {eq }}(\mathbf{P}, \mathbf{Q})$ depends on $(\mathbf{P}, \mathbf{Q})$ only through $\mathcal{H}_{0}$, we write:

$$
\langle\Delta B(t)\rangle=\int_{t_{0}}^{t} d t^{\prime} \mathcal{F}\left(t^{\prime}\right) \int d \mathbf{P} d \mathbf{Q} B(\mathbf{P}, \mathbf{Q}) e^{-i\left(t-t^{\prime}\right) \mathcal{L}_{0}}\left\{\mathcal{H}_{0}, A\right\} \frac{\partial}{\partial \mathcal{H}_{0}} f_{e q}\left(\mathcal{H}_{0}(\mathbf{P}, \mathbf{Q})\right) .
$$

Since $\frac{\partial}{\partial \mathcal{H}_{0}} f_{e q}\left(\mathcal{H}_{0}(\mathbf{P}, \mathbf{Q})\right)=-\beta f_{\text {eq }}\left(\mathcal{H}_{0}(\mathbf{P}, \mathbf{Q})\right)$ and $\left\{\mathcal{H}_{0}, A\right\}=-\frac{d A}{d t}$ we can write

$$
\langle\Delta B(t)\rangle=\beta \int_{t_{0}}^{t} d t^{\prime} \mathcal{F}\left(t^{\prime}\right) \int d \mathbf{P} d \mathbf{Q} B(\mathbf{P}, \mathbf{Q}) e^{-i\left(t-t^{\prime}\right) \mathcal{L}_{0}} \frac{d}{d t} A(\mathbf{P}, \mathbf{Q})_{t=0} f_{e q}(\mathbf{P}, \mathbf{Q}) .
$$

Finally, using the unitarity of the Liouvillian operator, we find:

$$
\langle\Delta B(t)\rangle=\beta \int_{t_{0}}^{t} d t^{\prime} \mathcal{F}\left(t^{\prime}\right) \int d \mathbf{P} d \mathbf{Q} \frac{d}{d t} A(\mathbf{P}, \mathbf{Q})_{t=t_{0}} f_{e q}(\mathbf{P}, \mathbf{Q}) e^{i\left(t-t^{\prime}\right) \mathcal{L}_{0}} B(\mathbf{P}, \mathbf{Q}) .
$$

If we introduce the response function, $R(t)$, through the relation

$$
\langle\Delta B(t)\rangle=\int_{t_{0}}^{t} d t^{\prime} R\left(t-t^{\prime}\right) \mathcal{F}\left(t^{\prime}\right),
$$

we may write

$$
R(t)=\beta \int d \mathbf{P} d \mathbf{Q} \frac{d}{d t} A(\mathbf{P}, \mathbf{Q})_{t=0} f_{e q}(\mathbf{P}, \mathbf{Q}) e^{i\left(t-t^{\prime}\right) \mathcal{L}_{0}} B(\mathbf{P}, \mathbf{Q})=\beta\left\langle\dot{A}\left(t_{0}\right) B(t)\right\rangle
$$

which can also be rewritten as

$$
R(t)=-\beta\left\langle A\left(t_{0}\right) \dot{B}(t)\right\rangle
$$

For later comparison, we can express the response function in a different guise, by defining the dissipative flux $J(\mathbf{P}, \mathbf{Q})$

$$
\begin{aligned}
& J(\mathbf{P}, \mathbf{Q}) f_{e q}(\mathbf{P}, \mathbf{Q})=-\left\{\mathcal{H}_{0}, A\right\} f_{e q}(\mathbf{P}, \mathbf{Q})=-\sum_{j}\left(\frac{\partial \mathcal{H}_{0}}{\partial q_{j}} K_{j}^{q}+\frac{\partial \mathcal{H}_{0}}{\partial p_{j}} K_{j}^{p}\right) f_{e q}(\mathbf{P}, \mathbf{Q}) \\
= & \sum_{j}\left(K_{j}^{q} F_{j}^{(0)}-K_{j}^{p} \frac{p_{j}}{m}\right) f_{e q}(\mathbf{P}, \mathbf{Q})
\end{aligned}
$$

where we have introduced the force acting on the $\mathrm{j}$-th particle, due to the Hamiltonian $\mathcal{H}_{0}$, as $F_{j}^{(0)}=-\frac{\partial \mathcal{H}_{0}}{\partial q_{j}}$. Using (2.17) we may write:

$$
R(t)=-\beta \int d \mathbf{P} d \mathbf{Q} J(\mathbf{P}, \mathbf{Q}) f_{e q}(\mathbf{P}, \mathbf{Q}) e^{i\left(t-t^{\prime}\right) \mathcal{L}_{0}} B(\mathbf{P}, \mathbf{Q})=-\beta\left\langle J\left(t_{0}\right) B(t)\right\rangle
$$




\subsection{Green-Kubo relations}

\subsubsection{Diffusion}

In order to probe the diffusive properties of a system of particles, we apply a spatially uniform force $h$ along an arbitrary direction, say the $x$-direction, and consider how the induced change of the average velocity depends on the external field. Due to the mutual interactions among the particles, this average velocity reaches a stationary value, because the drift current caused by the field is balanced by the diffusion current.

In order to achieve such a situation, we add the following perturbation to the Hamiltonian:

$$
A(\mathbf{P}, \mathbf{Q})=-h \sum_{j} q_{j}^{x}
$$

so that the dissipative flux, introduced above, turns out to be:

$$
J(\mathbf{P}, \mathbf{Q})=-\frac{h}{m} \sum_{j} p_{j}^{x} .
$$

We, now, compute the change of the average velocity along the $\mathrm{x}$-direction, i.e. we set $B(\mathbf{P}, \mathbf{Q})=U_{x}(\mathbf{P}, \mathbf{Q})=\frac{1}{m} \sum_{j} p_{j}^{x}$. By applying formula (2.18) we have

$$
\left\langle\Delta U_{x}(t)\right\rangle=-\beta \int_{t_{0}}^{t} d t^{\prime} \mathcal{F}\left(t^{\prime}\right) \int d \mathbf{P} d \mathbf{Q} J(\mathbf{P}, \mathbf{Q}) e^{-i\left(t-t^{\prime}\right) \mathcal{L}_{0}} U_{x}(\mathbf{P}, \mathbf{Q}) f_{e q}(\mathbf{P}, \mathbf{Q})
$$

or

$$
\begin{aligned}
\left\langle\Delta U_{x}(t)\right\rangle & =\beta \frac{h}{m^{2}} \int_{t_{0}}^{t} d t^{\prime} \mathcal{F}\left(t^{\prime}\right) \int d \mathbf{P} d \mathbf{Q} \sum_{j} p_{j}^{x} e^{-i\left(t-t^{\prime}\right) \mathcal{L}_{0}} \sum_{k} p_{k}^{x} f_{e q}(\mathbf{P}, \mathbf{Q}) \\
& =\beta \frac{h}{m^{2}} \int_{t_{0}}^{t} d t^{\prime} \mathcal{F}\left(t^{\prime}\right) \sum_{j k}\left\langle p_{j}^{x}\left(t_{0}\right) p_{k}^{x}\left(t-t^{\prime}\right)\right\rangle_{e q} .
\end{aligned}
$$

We, now, set $\mathcal{F}\left(t^{\prime}\right)=1$ for $t^{\prime} \geq 0, t_{0}=0$ and take into account the fact that the momenta of different particles are not correlated in the equilibrium system:

$$
\left\langle\Delta U_{x}(t)\right\rangle=\beta \frac{h}{m^{2}} \int_{0}^{t} d t^{\prime} \sum_{j}\left\langle p_{j}^{x}\left(t_{0}\right) p_{j}^{x}\left(t-t^{\prime}\right)\right\rangle_{e q} .
$$

At this stage, we connect the self-correlation function to the mobility, $\mu$, through the relation $\left\langle\Delta U_{x}(t)\right\rangle=\mu h$, to conclude that

$$
\mu=\frac{\beta}{m^{2}} \int_{0}^{\infty} d t^{\prime} \sum_{j}\left\langle p_{j}^{x}(0) p_{j}^{x}\left(t^{\prime}\right)\right\rangle_{e q} .
$$


Finally we obtain the diffusion constant from the Einstein relation $D=\mu / \beta$.

\subsubsection{Shear flow}

Let us now discuss how to calculate the coefficient of the shear viscosity. Assume the following form of the perturbation operator:

$$
A(\mathbf{P}, \mathbf{Q})=\frac{\gamma}{2} \sum_{j}\left(q_{j}^{y} p_{j}^{x}+q_{j}^{x} p_{j}^{y}\right)
$$

where $\gamma$ is the shear rate. The Hamiltonian with the above perturbation generates the following equations of motion:

$$
\begin{array}{lll}
\frac{\partial q_{j}^{x}}{\partial t}=\frac{p_{j}^{x}}{m}+\gamma q_{j}^{y} ; & \frac{\partial q_{j}^{y}}{\partial t}=\frac{p_{j}^{y}}{m}+\gamma q_{j}^{x} \\
\frac{\partial p_{j}^{x}}{\partial t}=F_{j}^{x}-\gamma p_{j}^{y} ; & \frac{\partial p_{j}^{y}}{\partial t}=F_{j}^{y}-\gamma p_{j}^{x}
\end{array}
$$

which are known as unthermostatted SLLOD equations describing a sheared fluid [17].

In this case the dissipative flux is:

$$
J(\mathbf{P}, \mathbf{Q})=\frac{\gamma}{2} \sum_{j}\left(\frac{2}{m} p_{j}^{x} p_{j}^{y}+q_{j}^{y} F_{j}^{x}+q_{j}^{x} F_{j}^{y}\right)
$$

and turns out to be proportional to the xy component of the pressure tensor

$$
P^{x y}=\frac{1}{2 V} \sum_{j}\left(\frac{2}{m} p_{j}^{x} p_{j}^{y}+q_{j}^{y} F_{j}^{x}+q_{j}^{x} F_{j}^{y}\right)
$$

via the relation $J(\mathbf{P}, \mathbf{Q})=\gamma V P^{x y}$, where $V$ is the volume of the system. By following steps similar to those leading to (2.24) we obtain

$$
\begin{aligned}
\left\langle\Delta P^{x y}(t)\right\rangle & =-\beta \int_{0}^{t} d t^{\prime} \int d \mathbf{P} d \mathbf{Q} J(\mathbf{P}, \mathbf{Q}) e^{-i\left(t-t^{\prime}\right) \mathcal{L}_{0}} P^{x y}(\mathbf{P}, \mathbf{Q}) f_{e q}(\mathbf{P}, \mathbf{Q}) \\
& =-\beta \gamma V \int_{0}^{t} d t^{\prime}\left\langle P^{x y}(0) P^{x y}\left(t^{\prime}\right)\right\rangle_{e q}
\end{aligned}
$$

where again we have used (2.18) and $\mathcal{F}\left(t^{\prime}\right)=1$ for $t^{\prime} \geq 0$.

Finally using the definition of the shear viscosity $\eta$

$$
\eta=-\lim _{t \rightarrow \infty} \frac{\left\langle\Delta P^{x y}(t)\right\rangle}{\gamma}
$$

we obtain:

$$
\eta=\beta V \int_{0}^{\infty} d t^{\prime}\left\langle P^{x y}(0) P^{x y}\left(t^{\prime}\right)\right\rangle_{e q} .
$$


Eqs. (2.24) and (2.32) are just two examples of more general relations known as Green-Kubo relations, connecting the transport coefficients to the equilibrium two-time correlations of a system.

A similar method is employed in order to derive relations for the thermal conductivity, $\lambda$, and the bulk viscosity coefficient, $\kappa$. The first of these relations reads:

$$
\lambda=\frac{1}{3 V k_{B} T^{2}} \int_{0}^{\infty} d t^{\prime}\left\langle\mathbf{S}(0) \cdot \mathbf{S}\left(t^{\prime}\right)\right\rangle_{e q}
$$

where

$$
\mathbf{S}=\sum_{j}\left(\frac{1}{2 m} p_{j}^{2}-<\epsilon_{j}>\right) \frac{\mathbf{p}_{j}}{m}+\frac{1}{2} \sum_{i} \sum_{j \neq i}\left(\mathbf{q}_{i j} \cdot \mathbf{F}_{i j}+U\left(\mathbf{q}_{i j}\right)\right) \frac{\mathbf{p}_{j}}{m}
$$

where $\left\langle\epsilon_{j}>\right.$ is the enthalpy per particle, $U\left(\mathbf{q}_{i j}\right)$ is the pair potential and $\mathbf{q}_{i j}=\mathbf{q}_{i}-\mathbf{q}_{j}$. For details see [18].

Similarly, the bulk viscosity reads:

$$
\kappa=\frac{1}{9 V k_{B} T} \int_{0}^{\infty} d t^{\prime} \sum_{a b}\left\langle\delta \mathcal{J}^{a a}(0) \delta \mathcal{J}^{b b}\left(t^{\prime}\right)\right\rangle_{e q}
$$

where $a$ and $b$ run over the spatial components,

$$
\mathcal{J}^{a b}=\sum_{j} \frac{p_{i}^{a} p_{i}^{b}}{m}+\sum_{j} q_{i}^{a} F_{i}^{b}
$$

and

$$
\delta \mathcal{J}^{a b}=\mathcal{J}^{a b}-\left\langle\mathcal{J}^{a b}\right\rangle
$$

\subsection{Linear Response for stochastic dynamics}

\subsubsection{Langevin Dynamics}

Previously we discussed only Hamiltonian systems perturbed by a weak timedependent external force. In the present section, we derive in a different fashion the FDR in systems subject to dissipative forces and random forces. Let us consider the following stochastic differential equation for the scalar variable $A(t)$

$$
\frac{d A(t)}{d t}=-\gamma \frac{\partial H[A(t)]}{\partial A(t)}+\sqrt{2 \gamma T} \eta(t)
$$

where $H[A(t)]$ is a function of $A(t)$ and $\eta$ is a white noise.

We define the two-time correlation function as:

$$
C\left(t, t^{\prime}\right)=\left\langle A(t) A\left(t^{\prime}\right)\right\rangle
$$


and assume without loss of generality $t>t^{\prime}$. In order to calculate the response of the system to a time dependent external perturbation, we consider the following perturbation

$$
H_{h}[A(t)]=H[A(t)]-h(t) A(t) .
$$

The variation of the quantity $A(t)$, induced by the presence of $h(t)$, is measured by the two-time response function, $R\left(t, t^{\prime}\right)$, defined as

$$
R\left(t, t^{\prime}\right)=\frac{\langle\delta A(t)\rangle}{\delta h\left(t^{\prime}\right)}
$$

The Fluctuation Dissipation theorem relates the two-time correlation function with the response function. Let us compute the following derivative of $C\left(t, t^{\prime}\right)$ :

$$
\frac{\partial C\left(t, t^{\prime}\right)}{\partial t}=\left\langle\left[-\gamma \frac{\partial H[A(t)]}{\partial A(t)}+\eta(t)\right] A\left(t^{\prime}\right)\right\rangle
$$

and subtract the derivative with respect to the argument $t^{\prime}$ :

$$
\frac{\partial C\left(t, t^{\prime}\right)}{\partial t}-\frac{\partial C\left(t, t^{\prime}\right)}{\partial t^{\prime}}=-\gamma\left\langle\frac{\partial H[A(t)]}{\partial A(t)} A\left(t^{\prime}\right)\right\rangle+\gamma\left\langle\frac{\partial H\left[A\left(t^{\prime}\right)\right]}{\partial A\left(t^{\prime}\right)} A(t)\right\rangle-\left\langle A(t) \eta\left(t^{\prime}\right)\right\rangle
$$

where we dropped a noise term because of causality. We use now the property, which is proved by Onsager's argument (cf. [19], pages 49-50):

$$
\langle A(t) B(t+\tau)\rangle=\langle A(t) B(t-\tau)\rangle=\langle A(t+\tau) B(t)\rangle
$$

the first equality derives from time reversal, the last equality follows from the invariance of the equilibrium state under a time translation $t \rightarrow t+\tau$. Therefore, the first two terms in the r.h.s. of eq. (2.43) cancel each other. Finally, the last term can be evaluated by means of Novikov's theorem [20] which states that if $\eta$ is a Gaussian process and $g$ is a function of $A(t)$ then

$$
\left\langle g(A(t)) \eta\left(t^{\prime}\right)\right\rangle=\int d t^{\prime \prime} \frac{\delta\langle g(A(t))\rangle}{\delta \eta\left(t^{\prime \prime}\right)}\left\langle\eta\left(t^{\prime \prime}\right) \eta\left(t^{\prime}\right)\right\rangle=\int d t^{\prime \prime} \frac{\delta\langle g(A(t))\rangle}{\delta h\left(t^{\prime \prime}\right)}\left\langle\eta\left(t^{\prime \prime}\right) \eta\left(t^{\prime}\right)\right\rangle .
$$

Setting $g(A(t))=A(t)$ we obtain, using eq.(2.41) and the property $\left\langle\eta\left(t^{\prime}\right) \eta\left(t^{\prime \prime}\right)\right\rangle=$ $\delta\left(t^{\prime}-t^{\prime \prime}\right)$

$$
\left\langle A(t) \eta\left(t^{\prime}\right)\right\rangle=\int d t^{\prime \prime} R\left(t, t^{\prime \prime}\right)\left\langle\eta\left(t^{\prime \prime}\right) \eta\left(t^{\prime}\right)\right\rangle=2 \gamma T R\left(t, t^{\prime}\right)
$$

and we find:

$$
\frac{\partial C\left(t, t^{\prime}\right)}{\partial t}-\frac{\partial C\left(t, t^{\prime}\right)}{\partial t^{\prime}}=-2 \gamma T R\left(t, t^{\prime}\right)
$$

At equilibrium, the two-time average depends only on the time difference, 
hence, setting $t^{\prime}=t+s$, we must have:

$$
\frac{\partial C(t, t+s)}{\partial t}=0
$$

or

so that we have

$$
\frac{\partial}{\partial t}\langle A(t) A(t+s)\rangle=-\frac{\partial}{\partial s}\langle A(t) A(t+s)\rangle
$$

$$
\begin{aligned}
& \frac{\partial C\left(t, t^{\prime}\right)}{\partial t}=-\frac{\partial C\left(t, t^{\prime}\right)}{\partial t^{\prime}} \\
& \frac{\partial C\left(t, t^{\prime}\right)}{\partial t^{\prime}}=\gamma T R\left(t, t^{\prime}\right) .
\end{aligned}
$$

We conclude that the response function has the form:

$$
R\left(t, t^{\prime}\right)=\frac{1}{\gamma T} \theta\left(t-t^{\prime}\right) \frac{\partial C\left(t, t^{\prime}\right)}{\partial t^{\prime}} .
$$

In order to connect this result with the static susceptibility, we switch on a constant perturbation $\epsilon$ at time $t=0$, and compute the change induced on the variable $A$, which is given, using relation (2.41), by the formula

$$
\langle A(t)\rangle-\langle A(0)\rangle=\epsilon \int_{0}^{t} d t^{\prime} R\left(t, t^{\prime}\right)=\epsilon \frac{1}{\gamma T}[C(t, t)-C(t, 0)] .
$$

After defining the integrated response $\chi$ by the formula:

$$
\chi(t, 0)=\frac{1}{\gamma T}[C(t, t)-C(t, 0)]
$$

we can verify that its $t \rightarrow \infty$ limit coincides with the static response function

$$
\chi_{s t}=\frac{1}{\gamma T}\left[\left\langle A^{2}\right\rangle-\langle A\rangle^{2}\right] .
$$

Here we have used the fact that the values of $A$ at time $t$ and at time 0 become uncorrelated as $t \rightarrow \infty$ and that the average factorizes.

\subsubsection{Linear Response in Fokker-Planck equation}

The probability distribution function of the process described by a stochastic differential equation evolves according to a Fokker-Planck equation. Within the Fokker-Planck formalism, we can generalize the method of section 2.1 to systems subjected to an average drift and a fluctuating term.

In such a case, the distribution $f(\mathbf{x}, t)$ evolves according to:

$$
\frac{\partial}{\partial t} f(\mathbf{x}, t)=\left(\mathcal{L}_{F P}^{0}+\mathcal{L}_{F P}^{e x t}(t)\right) f(\mathbf{x}, t)
$$


where $\mathcal{L}_{F P}^{0}$ represents the Fokker-Planck operator of the unperturbed system, defined by

$$
\mathcal{L}_{F P}^{0} f(\mathbf{x}, t)=-\sum_{j} \partial_{j}\left[D_{j}^{(1)}(\mathbf{x}) f(\mathbf{x}, t)\right]+\sum_{j k} \partial_{j} \partial_{k} D_{j k}^{(2)}(\mathbf{x}) f(\mathbf{x}, t)
$$

where the functions $D_{j}^{(1)}$ and $D_{j k}^{(2)}$ are related to the underlying Ito stochastic differential equations by

$$
\frac{d x_{j}(t)}{d t}=D_{j}^{(1)}(\mathbf{x})+\sum_{k} M_{j k}(\mathbf{x}) \eta_{k}(t),
$$

and

$$
D_{j k}^{(2)}(\mathbf{x})=\frac{1}{2} M_{j l}(\mathbf{x}) M_{l k}^{T}(\mathbf{x})
$$

where the white noise, $\eta_{k}$, is a Gaussian process with

$$
\left\langle\eta_{k}(t)\right\rangle=0, \quad\left\langle\eta_{j}(t) \eta_{k}\left(t^{\prime}\right)\right\rangle=\delta_{j k} \delta\left(t-t^{\prime}\right) .
$$

We probe the response of the system, by adding an infinitesimal time-dependent perturbation of the form

$$
\delta D_{j}^{(1)}(\mathbf{x}, t)=\mathcal{F}(t) K_{j}(\mathbf{x}) .
$$

Such a perturbation induces the following change in the Fokker-Planck operator:

$$
\mathcal{L}_{F P}^{e x t}(t) f(\mathbf{x}, t)=-\mathcal{F}(t) \sum_{j} \partial_{j}\left(K_{j}(\mathbf{x}) f(\mathbf{x}, t)\right)
$$

We first determine the perturbed distribution, assuming that the system was stationary in the infinite past, i.e.: $f(\mathbf{x},-\infty)=f_{s t}(\mathbf{x})$ and

$$
\mathcal{L}_{F P}^{0} f^{s t}(\mathbf{x})=0 \text {. }
$$

An approximate solution of the perturbed problem

$$
\frac{\partial}{\partial t} f(\mathbf{x}, t)=\left[\mathcal{L}_{F P}^{0}+\mathcal{L}_{F P}^{e x t}(t)\right] f(\mathbf{x}, t)
$$

to first order in the perturbation $\mathcal{L}_{F P}^{e x t}$ can be represented as: $f(\mathbf{x}, t) \simeq f^{s t}(\mathbf{x})+$ $\Delta f(\mathbf{x}, t)$, where $\Delta f$ is formally given by:

$$
\Delta f(\mathbf{x}, t)=\int_{-\infty}^{t} d t^{\prime} e^{\left(t-t^{\prime}\right) \mathcal{L}_{F P}^{0}} \mathcal{L}_{F P}^{e x t}\left(t^{\prime}\right) f^{s t}(\mathbf{x}) .
$$

With this approximation, the deviation of the noise average of a physical quantity $B(\mathbf{x})$, due to the perturbation (2.61), can be written as:

$$
\langle\Delta B(t)\rangle=\langle B(\mathbf{x}, t)\rangle-\langle B(\mathbf{x}, t=-\infty)\rangle=\int d^{N} x B(\mathbf{x}) \Delta f(\mathbf{x}, t)
$$


or, substituting eq. (2.65),

$$
\langle\Delta B(t)\rangle=\int_{-\infty}^{t} d t^{\prime} \int d^{N} x B(\mathbf{x}) e^{\mathcal{L}_{F P}^{0}\left(t-t^{\prime}\right)} \mathcal{L}_{F P}^{e x t}\left(t^{\prime}\right) f^{s t}(\mathbf{x})
$$

compactly written as

$$
\Delta B(t)=\int_{-\infty}^{t} d t^{\prime} R\left(t-t^{\prime}\right) \mathcal{F}\left(t^{\prime}\right)
$$

where the response function $R\left(t-t^{\prime}\right)$, for $t \geq t^{\prime}$, reads:

$$
R(t)=-\int d^{N} x B(\mathbf{x}) e^{\mathcal{L}_{F P}^{0} t} \sum_{j} \partial_{j}\left(K_{j}(\mathbf{x}) f^{s t}(\mathbf{x})\right)
$$

and $R(t)=0$ for $t<0$, where (2.62) has been used.

In order to relate the response function to the correlation in the unperturbed case, between two operators $B(\mathbf{x}, t)$ and $\tilde{B}\left(\mathbf{x}^{\prime}, t^{\prime}\right)$, defined as:

$$
\left\langle B(t) \tilde{B}\left(t^{\prime}\right)\right\rangle=\iint d^{N} x d^{N} x^{\prime} B(\mathbf{x}) \tilde{B}\left(\mathbf{x}^{\prime}\right) W_{2}\left(\mathbf{x}, t ; \mathbf{x}^{\prime}, t^{\prime}\right)
$$

where $W_{2}\left(\mathbf{x}, t ; \mathbf{x}^{\prime}, t^{\prime}\right)$ is the joint probability distribution for the unperturbed case, we use the result:

$$
W_{2}\left(\mathbf{x}, t ; \mathbf{x}^{\prime}, t^{\prime}\right)=P\left(\mathbf{x}, t, \mathbf{x}^{\prime}, t^{\prime}\right) f^{s t}\left(\mathbf{x}^{\prime}\right)=e^{\mathcal{L}_{F P}^{0}\left(t-t^{\prime}\right)} \delta\left(\mathbf{x}-\mathbf{x}^{\prime}\right) f^{s t}\left(\mathbf{x}^{\prime}\right)
$$

where $P\left(\mathbf{x}, t, \mathbf{x}^{\prime}, t^{\prime}\right)$ is the transition probability from configuration $\mathbf{x}^{\prime}$ to $\mathbf{x}$. Substituting such an expression in (2.70) we obtain

$$
\begin{aligned}
\left\langle B(t) \tilde{B}\left(t^{\prime}\right)\right\rangle=\int & d^{N} x\left[\theta\left(t-t^{\prime}\right) B(\mathbf{x}) e^{\mathcal{L}_{F P}^{0}\left(t-t^{\prime}\right)} \tilde{B}(\mathbf{x})\right. \\
+ & \left.\theta\left(t^{\prime}-t\right) \tilde{B}(\mathbf{x}) e^{-\mathcal{L}_{F P}^{0}\left(t-t^{\prime}\right)} B(\mathbf{x})\right] f^{s t}(\mathbf{x})
\end{aligned}
$$

Hence, provided we make the identification

$$
\tilde{B}\left(t^{\prime}\right)=\beta \frac{d A\left(t^{\prime}\right)}{d t^{\prime}}=-\frac{1}{f^{s t}(\mathbf{x})} \sum_{j} \partial_{j}\left(K_{j}(\mathbf{x}) f^{s t}(\mathbf{x})\right)
$$

we can rewrite the response function as:

$$
R(t)=\beta \int d^{N} x B(\mathbf{x}) e^{\mathcal{L}_{F P}^{0} t} \frac{d A(\mathbf{x})}{d t} f^{s t}(\mathbf{x})=\beta\left\langle B(t)\left(\frac{d A(t)}{d t}\right)_{t=0}\right\rangle,
$$

so that the analogy with the Hamiltonian formulation of the FDR is complete. 


\subsubsection{Kramers equation}

As an important special case, we consider the motion of a colloidal particle in an external field. To obtain a more useful expression and make contact with the FDR in Hamiltonian systems, we assume the Fokker-Planck equation to be of the form of a Kramers equation. Such stochastic dynamics can be con-

sidered as a Hamiltonian dynamical system, in which the momentum equation is augmented by a friction and a noise term. Denoting $\mathbf{x}=\left(p_{j}, q_{j}\right)$, we have

$$
\begin{aligned}
\frac{d q_{j}}{d t} & =\frac{\partial \mathcal{H}_{0}}{\partial p_{j}}+\mathcal{F}(t) K_{j}^{q} \\
\frac{d p_{j}}{d t} & =-\frac{\partial \mathcal{H}_{0}}{\partial q_{j}}-\gamma p_{j}+\mathcal{F}(t) K_{j}^{p}+\sqrt{2 \gamma m k_{B} T} \eta_{j} .
\end{aligned}
$$

The associated linear evolution operators are

$$
\mathcal{L}_{F P}^{0}=-\sum_{j}\left(\frac{\partial \mathcal{H}_{0}}{\partial p_{j}} \frac{\partial}{\partial q_{j}}-\frac{\partial \mathcal{H}_{0}}{\partial q_{j}} \frac{\partial}{\partial p_{j}}\right)+\gamma \sum_{j}\left(\frac{\partial}{\partial p_{j}} p_{j}-\beta^{-1} \frac{\partial^{2}}{\partial p_{j}^{2}}\right)
$$

and

$$
\mathcal{L}_{F P}^{e x t}(t)=-\mathcal{F}(t) \sum_{j}\left(K_{j}^{q} \frac{\partial}{\partial q_{j}}+K_{j}^{p} \frac{\partial}{\partial p_{j}}\right) .
$$

When $\mathcal{F}(t)=0$ the system has an equilibrium distribution $f^{s t}(\mathbf{P}, \mathbf{Q})=$ $\mathcal{N} \exp \left(-\beta \mathcal{H}_{0}\right)$, so that we may explicitly compute the expression:

$$
\begin{aligned}
\sum_{j} K_{j}(\mathbf{P}, \mathbf{Q}) \partial_{j} f^{s t}(\mathbf{P}, \mathbf{Q}) & =-\beta\left(K_{j}^{q} \frac{\partial \mathcal{H}_{0}}{\partial q_{j}}+K_{j}^{p} \frac{\partial \mathcal{H}_{0}}{\partial p_{j}}\right) f^{s t}(\mathbf{P}, \mathbf{Q}) \\
& =\beta\left(K_{j}^{q} F_{j}-K_{j}^{p} \frac{p_{j}}{m}\right) f^{s t}(\mathbf{P}, \mathbf{Q}) \\
& =J(\mathbf{P}, \mathbf{Q}) f^{s t}(\mathbf{P}, \mathbf{Q})
\end{aligned}
$$

where the dissipative flux $J(\mathbf{P}, \mathbf{Q})$ is identical to the one in Eq. (2.17).

By comparing eq. (2.80) and (2.69) one sees that, for $t>0, R(t)$ can be expressed as the two-time correlation:

$$
R(t)=-\beta \int d^{N} x B(\mathbf{P}, \mathbf{Q}) e^{\mathcal{L}_{F P}^{0} t} J(\mathbf{P}, \mathbf{Q}) f^{s t}(\mathbf{P}, \mathbf{Q})
$$

which is similar to eq. (2.18), provided $\mathcal{L}_{F P}^{0}$ is replaced by $-i \mathcal{L}^{0}$. 


\subsubsection{Smoluchowski equation}

The Smoluchowski equation can be considered the Fokker-Planck (FP) equation which arises in the overdamped limit, $\gamma \rightarrow \infty$, of Langevin's equation $(2.76)$ :

$$
\frac{d q_{j}}{d t}=-\Gamma \frac{\partial \mathcal{H}_{0}}{\partial q_{j}}+\sqrt{2 \Gamma k_{B} T} \eta_{j}
$$

with $\Gamma=1 / m \gamma$. The associated $\mathrm{FP}$ equation reads:

$$
\frac{\partial}{\partial t} f(\{q\}, t)=\left(\mathcal{L}_{S}^{0}+\mathcal{L}_{S}^{e x t}(t)\right) f(\{q\}, t)
$$

where $\mathcal{L}_{S}^{0}=\Gamma \sum_{j} \partial_{j}\left(\partial_{j}+\beta \partial_{j} \mathcal{H}_{0}\right)$ and $\mathcal{L}_{S}^{e x t}(t)=-\Gamma \mathcal{F}(t) \sum_{j} K_{j} \partial_{j}$. In this case we find that the current is determined by the following condition:

$$
\begin{aligned}
\sum_{j} K_{j}(\{q\}) \partial_{j} f^{s t}(\{q\}) & =-\beta K_{j} \frac{\partial \mathcal{H}_{0}}{\partial q_{j}} f^{s t}(\{q\}) \\
& =\beta K_{j} F_{j} f^{s t}(\{q\})=J(\{q\}) f^{s t}(\{q\})
\end{aligned}
$$

with $f^{s t}(\{q\})=\mathcal{N} \exp \left(-\beta \mathcal{H}_{0}^{\text {config }}(\{q\})\right)$. We conclude that in this case the response function is:

$$
R(t)=-\beta \int d^{N} q B(\{q\}) e^{\mathcal{L}_{S}^{0} t} J(\{q\}) f^{s t}(\{q\}) .
$$

\subsection{Johnson-Nyquist spectrum and Onsager regression hypothesis}

Even before Onsager's and Kubo's great synthesis, important results had been obtained in some specific cases or under special conditions. Such contributions played a significant role in the development of statistical physics. In the following we illustrate in detail some of them.

\subsubsection{Frequency response and Johnson-Nyquist spectrum}

Equations (2.15)-(2.16), relating the response to a given perturbation and a suitable equilibrium correlation, is often useful in its frequency representation. If the external perturbation is periodic in time,

$$
\mathcal{F}(t)=\operatorname{Re}\left[\mathrm{K}_{0} \mathrm{e}^{\mathrm{i} \omega \mathrm{t}}\right]
$$

because of linearity, the response reads

$$
\langle\Delta B(t)\rangle=\operatorname{Re}\left[\chi(\omega) \mathrm{K}_{0} \mathrm{e}^{\mathrm{i} \omega \mathrm{t}}\right]
$$


where $\chi(\omega)$ is the admittance defined as the Fourier transform of the response function $R(t)$ :

$$
\chi(\omega)=\int_{-\infty}^{\infty} R(t) \mathrm{e}^{-i \omega t} d t=\beta \int_{t_{0}}^{\infty}\left\langle\dot{A}\left(t_{0}\right) B(t)\right\rangle \mathrm{e}^{-i \omega t} d t .
$$

As an interesting example, we discuss the generalized Langevin equation, with a retarded frictional force $\gamma(t)$ :

$$
\frac{d u(t)}{d t}=-\int_{t_{0}}^{t} \gamma\left(t-t^{\prime}\right) u\left(t^{\prime}\right) d t^{\prime}+\frac{1}{m} \eta(t)+\frac{1}{m} \mathcal{F}(t), \quad t>t_{0}
$$

where the external force $\mathcal{F}(t)$ is also included in this equation. The usual linear Langevin equation is the limiting case with $\gamma(t)=\gamma \delta(t)$. It is immediate to see that the response of $u(t)$ to a periodic perturbation of the form (2.86) reads

$$
\langle u(t)\rangle=\operatorname{Re}\left[\chi(\omega) \mathrm{K}_{0} \mathrm{e}^{\mathrm{i} \omega \mathrm{t}}\right],
$$

where the admittance is given by

$$
\chi(\omega)=\frac{1}{m} \frac{1}{i \omega+\tilde{\gamma}(\omega)},
$$

with $\tilde{\gamma}(\omega)$ the Fourier transform of $\gamma(t)$. In other words, for a periodic perturbation, the admittance is analogous to the mobility. At equilibrium, $\mathcal{F} \equiv 0$, the self-correlation of $u(t)$ can be calculated, and its Fourier transform reads

$$
\int_{0}^{\infty} \mathrm{e}^{-i \omega t}\left\langle u\left(t_{0}\right) u\left(t_{0}+t\right)\right\rangle d t=\left\langle u^{2}\right\rangle \frac{1}{i \omega+\tilde{\gamma}(\omega)}
$$

and therefore the FDR

$$
\chi(\omega)=\frac{1}{m\left\langle u^{2}\right\rangle} \int_{0}^{\infty}\left\langle u\left(t_{0}\right) u\left(t_{0}+t\right)\right\rangle \mathrm{e}^{-i \omega t} d t
$$

is recovered. Note also that Eq. (2.92) allows us to calculate the power spectrum of $u(t)$ at equilibrium, i.e.

$$
S_{u}(\omega)=\int_{-\infty}^{\infty} \mathrm{e}^{-i \omega t}\left\langle u\left(t_{0}\right) u\left(t_{0}+t\right)\right\rangle d t=\left\langle u^{2}\right\rangle \frac{2 \tilde{\gamma}(\omega)}{[\tilde{\gamma}(\omega)]^{2}+\omega^{2}}
$$

A particular case of Eq. (2.89) is the equation governing the charge $Q(t)$ contained in a condenser of capacity $C$, in a simple $R C$ circuit without any externally applied electromotive force:

$$
R \frac{d Q}{d t}=-\frac{Q(t)}{C}+\eta(t)
$$


being $\eta(t)$ a noisy internal electromotive force due to the interactions with a thermostat at temperature T. A series of experiments by Johnson [21], supported by the theory of Nyquist [22], demonstrated the existence of such spontaneous fluctuations of electric tension at the edges of the resistor $u=Q / C$, due to the thermal motion of charge carriers. The measured quantity in the experiment was the power spectrum of these tension fluctuations, i.e. $S_{u}(\omega)$. The above equation for the $R C$ circuit can be written in the form

$$
\frac{d u}{d t}=-\frac{u(t)}{R C}+\eta^{\prime}(t)
$$

with $\eta^{\prime}(t)=\eta / R C$. This is equivalent to Eq. (2.89) with $\gamma(t)=\frac{1}{R C} \delta(t)$, i.e. $\tilde{\gamma}(\omega)=\frac{1}{R C}$, resulting in

$$
S_{u}(\omega)=\left\langle u^{2}\right\rangle \frac{2 R C}{1+(R C \omega)^{2}}
$$

The energy of the capacitor is $E=Q^{2} / 2 C$, and the equipartition of energy, required at equilibrium, imposes that $\langle E\rangle=\frac{k_{B} T}{2}$, which yields $\left\langle u^{2}\right\rangle=\left\langle Q^{2}\right\rangle / C^{2}=$ $2\langle E\rangle / C=k_{B} T / C$. This result, put into Eq. (2.97), gives the spectrum of the Johnson-Nyquist noise

$$
S_{u}(\omega)=\frac{2 R k_{B} T}{1+(\omega R C)^{2}} .
$$

At low frequencies, $\omega \ll 1 / R C$, the power spectrum becomes constant and does not depend on the capacity of the circuit:

$$
S_{u}(\omega) \simeq 2 R k_{B} T
$$

For a modern linear response study of chaotic systems in frequency domain we refer the reader to [23].

\subsubsection{The Onsager regression hypothesis}

In his two seminal papers of 1931 [24,25], Onsager presented his regression hypothesis: "... the average regression of fluctuations will obey the same laws as the corresponding macroscopic irreversible process" [25]. This principle states the equivalence between the law governing the relaxation of spontaneous deviations from equilibrium and the law obeyed by the corresponding irreversible process, which is triggered by an initial small deviation from equilibrium. The fluctuation-dissipation theorem (2.16), justifies this hypothesis in a natural way.

Onsager referred to the relaxation of a macroscopic variable $\alpha(t)$, an observable function of the microscopic degrees of freedom, $\alpha(\mathbf{P}(t), \mathbf{Q}(t))$, whose equilib- 
rium average, $\alpha_{0}$ is given by

$$
\alpha_{0}=\langle\alpha(\mathbf{P}, \mathbf{Q})\rangle=\frac{\int d \mathbf{P} d \mathbf{Q} e^{-\beta \mathcal{H}(\mathbf{P}, \mathbf{Q})} \alpha(\mathbf{P}, \mathbf{Q})}{\int d \mathbf{P} d \mathbf{Q} e^{-\beta \mathcal{H}(\mathbf{P}, \mathbf{Q})}}
$$

with the unperturbed Hamiltonian $\mathcal{H}(\mathbf{P}, \mathbf{Q})$. Fluctuations around this value are denoted by $\delta \alpha(t)=\alpha(t)-\alpha_{0}$. The average regression at time $t_{1}$ of a fluctuation $\delta \alpha\left(t_{0}\right)$, at time $t_{0}$, is given by the auto-correlation function $\left\langle\delta \alpha\left(t_{0}\right) \delta \alpha\left(t_{1}\right)\right\rangle$. The self-correlation function depends only on the difference $t_{1}-t_{0}$, since we are considering a time-translation invariant (equilibrium) state, therefore in the following we take $t_{0}=0$ and $t_{1}=t$.

The irreversible processes considered by Onsager, on the other hand, consist of the average evolution of the observable $\alpha(t)$, with $t>0$, when a small perturbation $\Delta \mathcal{H}$ is applied to the Hamiltonian from time $-\infty$ up to time 0 , and then is switched off. This corresponds to a perturbation of the Hamiltonian $\Delta \mathcal{H}=-\mathcal{F} \alpha(\mathbf{P}, \mathbf{Q})$, so that the conjugated field of the perturbation is $\alpha(\mathbf{P}, \mathbf{Q})$ itself. The average evolution for times $t>0$ (the so-called "after-effect function") is given by

$$
\bar{\alpha}(t)=\frac{\int d \mathbf{P} d \mathbf{Q} e^{-\beta \mathcal{H}(\mathbf{P}, \mathbf{Q})+\Delta \mathcal{H}(\mathbf{P}, \mathbf{Q})} \alpha(\mathbf{P}, \mathbf{Q})}{\int d \mathbf{P} d \mathbf{Q} e^{-\beta \mathcal{H}(\mathbf{P}, \mathbf{Q})+\Delta \mathcal{H}(\mathbf{P}, \mathbf{Q})}} .
$$

Furthermore, the perturbation $\mathcal{F}(t)=F \Theta(-t)$ is such that $\bar{\alpha}(0)-\alpha_{0}=\delta \alpha(0)$. The regression hypothesis can therefore be formulated in the following way:

$$
\frac{\bar{\alpha}(t)-\alpha_{0}}{\bar{\alpha}(0)-\alpha_{0}}=\frac{\langle\delta \alpha(t) \delta \alpha(0)\rangle}{\left\langle(\delta \alpha(0))^{2}\right\rangle}=\frac{\langle\alpha(t) \alpha(0)\rangle-\alpha_{0}^{2}}{\left\langle\alpha^{2}\right\rangle-\alpha_{0}^{2}} .
$$

By definition, the irreversible evolution in the l.h.s. is governed by macroscopic linear laws, as discussed in Onsager's two 1931 papers, so that the fluctuationdissipation theorem (2.16) can be used to compute $\bar{\alpha}(t)$. For this purpose, it is convenient to consider $\mathcal{H}+\Delta \mathcal{H}$ as the unperturbed Hamiltonian, with ensemble averages given by $\langle\ldots\rangle_{\Delta \mathcal{H}}$, and $-\Delta \mathcal{H}$ as the perturbation switched on at time 0 . From (2.16) one gets:

$$
\begin{aligned}
& \bar{\alpha}(t)=\langle\alpha\rangle_{\Delta \mathcal{H}}+\beta F \int_{0}^{t} d t^{\prime}\left\langle\alpha(0) \dot{\alpha}\left(t-t^{\prime}\right)\right\rangle_{\Delta \mathcal{H}}= \\
&\langle\alpha\rangle_{\Delta \mathcal{H}}+\beta F\left[\langle\alpha(t) \alpha(0)\rangle_{\Delta \mathcal{H}}-\left\langle\alpha^{2}\right\rangle_{\Delta \mathcal{H}}\right]
\end{aligned}
$$

At $t=0$ one has $\langle\alpha\rangle_{\Delta \mathcal{H}}=\alpha_{0}+\delta \alpha(0)$. Asymptotically, for $t \rightarrow+\infty$, one has $\bar{\alpha}(t) \rightarrow \alpha_{0}$, and $\langle\alpha(t) \alpha(0)\rangle_{\Delta \mathcal{H}} \rightarrow\left(\langle\alpha\rangle_{\Delta \mathcal{H}}\right)^{2}$, which, substituting in (2.103), gives $\beta F\left\langle\alpha^{2}\right\rangle_{\Delta \mathcal{H}}=\beta F\left(\langle\alpha\rangle_{\Delta \mathcal{H}}\right)^{2}+\delta \alpha(0)$. With these relations, Eq. (2.103) becomes

$$
\bar{\alpha}(t)=\alpha_{0}+\beta F\left[\langle\alpha(t) \alpha(0)\rangle_{\Delta \mathcal{H}}-\left(\langle\alpha\rangle_{\Delta \mathcal{H}}\right)^{2}\right]
$$


It is now plain that, to be consistent with the linear approximation, all the effects of the perturbation $\Delta \mathcal{H}$ can be neglected in the functions appearing in the r.h.s, which are of second order in $\alpha$. This means that all the subscripts in the perturbed averages $\langle\ldots\rangle_{\Delta \mathcal{H}}$ can be dropped and formula (2.104), i.e. the regression hypothesis, turns out to be a particular case of the FluctuationDissipation theorem.

\subsubsection{The Onsager reciprocal relations}

In $[24,25]$, Onsager derived a relation among off-diagonal elements of the evolution matrix for linear irreversible processes. It was, in fact, the central result of that work. Again, it is possible, a posteriori, to offer a simpler derivation of that relation, starting from the Green-Kubo formulas. As we have seen, a direct consequence of FDR is the possibility to write the transport coefficients as time-integrals of suitable correlation functions. In general, if one has $N$ conserved quantities, $\alpha_{i}(\mathbf{x}, t), i=(1, . . N)$, and small spatial and temporal variations of the fields, a linear system of evolution equations can be written as

$$
\begin{aligned}
\frac{\partial \alpha_{i}(\mathbf{x}, t)}{\partial t} & =-\nabla \cdot \mathbf{J}_{i}(\mathbf{x}, t) \\
\mathbf{J}_{i}(\mathbf{x}, t) & =L_{i j} \boldsymbol{\chi}_{j},
\end{aligned}
$$

where the fluxes $\mathbf{J}_{i}$, the transport coefficients $L_{i j}$ and the thermodynamic forces $\chi_{j}$ have been introduced. The latter are the forces conjugated to the fluxes in the dissipation function (entropy production) of non-equilibrium thermodynamics [26]. A thermodynamic force $\boldsymbol{\chi}_{j}$ is in general a gradient of the same quantity $\alpha_{i}(\mathbf{x}, t)$ : this is the application of linear response theory to internal forces, in fact the fluxes are responses to internal gradients. For instance, the heat flux $q_{x}$ along the $\hat{x}$ direction is expressed as $q=-\kappa \partial_{x} T$ with $\kappa$ the thermal conductivity. Note that for each flux-force pair $(i, j)$, the coefficient $L_{i j}$ is indeed a matrix of indexes $(k, l)$ for all possible pairs of the Cartesian components, i.e. $L_{i j}=\left(L_{i j}^{k l}\right)$ with $k, l=x, y, z$. We recall the Green-Kubo relations for these coefficients:

$$
L_{i j}^{k l}=\beta V \int_{0}^{\infty} d s\left\langle J_{i}^{k}(t) J_{j}^{l}(0)\right\rangle
$$

Onsager's reciprocal relations between $L_{i j}^{k l}$ and $L_{j i}^{l k}$ appears as a consequence of microscopic time reversibility. The equations of motion for the evolution of a phase space point (describing a system of $N$ particles) are invariant for the time reversal operation $I$, which transforms $\mathbf{Q} \rightarrow \mathbf{Q}, \mathbf{P} \rightarrow-\mathbf{P}$ and $t \rightarrow-t$. This invariance implies the invariance of the phase space probability measure. Denoting by $S^{t}$ the time evolution operator, which satisfies $I S^{t}=S^{-t} I$, the 
following chain of identities holds for a generic time correlation function:

$$
\begin{aligned}
\left\langle\left[S^{t} J_{i}^{k}\right] J_{j}^{l}\right\rangle=\left\langle I\left\{\left[S^{t} J_{i}^{k}\right] J_{j}^{l}\right\}\right\rangle=\left\langle\left[S^{-t} I J_{i}^{k}\right] I J_{j}^{l}\right\rangle \\
=\left\langle\left[S^{t} I J_{j}^{l}\right] I J_{i}^{k}\right\rangle=I_{i} I_{j}\left\langle\left[S^{t} J_{j}^{l}\right] J_{i}^{k}\right\rangle
\end{aligned}
$$

where the invariance to a translation of time has also been used. In the last passage, we have exploited the fact that the fluxes have the opposite parity of their conjugated density under time reversal, so that $I J_{i}^{k}=I_{i} J_{i}^{k}$ where $I_{i}= \pm 1$, for example heat and mass fluxes are odd, while the momentum flux is even. From the above identities, the reciprocal relations follow:

$$
L_{i j}^{k l}=I_{i} I_{j} L_{j i}^{l k}
$$

\section{Fluctuation dissipation in chaotic systems and statistical physics}

The Fluctuation-Response theory was originally developed in the context of equilibrium statistical mechanics of Hamiltonian systems $[27,28]$. In this Section we will show that a generalized FDR holds under rather general hypotheses[29,30,31,32]: hence also for non-Hamiltonian systems. For instance, it holds for a vast class of systems with chaotic dynamics, something that is particularly relevant in hydrodynamics[33,34].

Usually the Fluctuation-Response problem has been studied for infinitesimal perturbations; in many problems of statistical mechanics this is not a serious limitation. However, it is possible to see that a FD relation holds also for finite perturbations. This is important in different contexts, e.g. in geophysical or climate investigations, where the study of small perturbations is rather academic, while the relaxation of large fluctuations due to fast changes of the parameters is an issue of practical relevance [35]. In the following we derive a FDR which is valid independently of the number of degrees of freedom.

\subsection{Chaos and the FDR: van Kampen's objection}

Van Kampen argued that the usual derivation of the FDR, based on a firstorder truncation of the time-dependent perturbation theory for the evolution of probability density, is not fully justified [36]. In a nutshell, using the dynamical systems terminology, van Kampen's argument can be summarized as follows. Given a perturbation $\delta \mathbf{x}(0)$, on the state of the system $\mathbf{x}(0)$ at time 0 , one can write a Taylor expansion for the difference between the perturbed 
and the unperturbed trajectories at time $t, \delta \mathbf{x}(t)$, whose components are:

$$
\delta x_{i}(t)=\sum_{j} \frac{\partial x_{i}(t)}{\partial x_{j}(0)} \delta x_{j}(0)+O\left(|\delta \mathbf{x}(0)|^{2}\right)
$$

After averaging over the initial conditions, one obtains the mean response function:

$$
R_{i, j}(t)=\left\langle\frac{\partial x_{i}(t)}{\partial x_{j}(0)}\right\rangle=\int \frac{\partial x_{i}(t)}{\partial x_{j}(0)} \rho(\mathbf{x}(0)) d \mathbf{x}(0)
$$

In a Hamiltonian system one has $\rho(\mathbf{x}) \propto \exp (-\beta H(\mathbf{x}))$, so that, integrating by parts, we obtain

$$
R_{i, j}(t)=\beta\left\langle x_{i}(t) \frac{\partial H(\mathbf{x}(0))}{\partial x_{j}(0)}\right\rangle
$$

which is nothing but the usual FDR $[27,28]$.

In the presence of chaos the terms $\partial x_{i}(t) / \partial x_{j}(0)$ grow exponentially as $e^{\lambda t}$, where $\lambda$ is the Lyapunov exponent, therefore it is not possible to use the linear expansion (3.1) for a time larger than $(1 / \lambda) \ln (L /|\delta \mathbf{x}(0)|)$, where $L$ is the typical fluctuation size of the variable $\mathbf{x}$. Such an argument suggests that the linear response theory is valid only for extremely small and unphysical perturbations or for short times. Indeed, according to van Kampen's argument, the FDR holds up to $1 s$ if a perturbing electric field smaller than $10^{-20} \mathrm{~V} / \mathrm{m}$ is applied to the electrons of a conductor, in clear disagreement with experience.

The possible repercussions of van Kampen's arguments are summarized by the following two sentences [36]: The basic linearity assumption of linear theory is shown to be completely unrealistic and incompatible with basic ideas of statistical mechanics of irreversible processes; and Linear response theory does provide expressions for the phenomenological coefficients, but I assert that it arrives at these expressions by a mathematical exercise, rather than by describing the actual mechanism which is responsible for the response. These observations stimulated further research which resulted in a deeper understanding of the physical mechanisms underlying the validity of the FDR and of its range of applicability.

As a matter of fact, the success of the linear theory for the computation of the transport coefficients (e.g. electric conductivity), in terms of correlation functions of the unperturbed systems, is transparent, and its validity has been, directly and indirectly, tested in a huge variety of cases. To reconcile van Kampen's argument with the well established linear response theory, note that van Kampen considered single trajectories, while the FDR deals with observables quantities, which are averages over ensembles of trajectories, and have a much more regular behavior than the single trajectories. Indeed, the reason of the seemingly inexplicable effectiveness of the Linear-Response theory lies in the 
"constructive role of chaos" because, as Kubo observed, "instability lof the trajectories] instead favors the stability of distribution functions, working as the cause of the mixing" [28].

\subsection{Generalized FDR for non Hamiltonian systems}

In the following we will give a derivation, under rather general hypotheses, of a general FDR, which avoids van Kampen's critique. Consider a dynamical system $\mathbf{x}(0) \rightarrow \mathbf{x}(t)=S^{t} \mathbf{x}(0)$ with states $\mathbf{x}$ belonging to a $N$-dimensional vector space. For the sake of generality, we consider the possibility that the time evolution be not completely deterministic (e.g. it could be described by stochastic differential equations). We assume that the system is mixing and that the invariant probability distribution $\rho(\mathbf{x})$ enjoys some regularity property (see later), while no assumption is made on $N$.

Our aim is to express the average response of a generic observable $A$ to a perturbation, in terms of suitable correlation functions, computed according to the invariant measure of the unperturbed system. The first step is to study the behavior of a single component of $\mathbf{x}$, say $x_{i}$, when the system, described by $\rho(\mathbf{x})$, is subjected to an initial (non-random) perturbation $\mathbf{x}(0) \rightarrow$ $\mathbf{x}(0)+\Delta \mathbf{x}_{0} 11$. This instantaneous kick modifies the density of the system into $\rho^{\prime}(\mathbf{x})$, which is related to the invariant distribution by $\rho^{\prime}(\mathbf{x})=\rho\left(\mathbf{x}-\Delta \mathbf{x}_{0}\right)$. We introduce the probability of transition from $\mathbf{x}_{0}$ at time 0 to $\mathbf{x}$ at time $t$, $W\left(\mathbf{x}_{0}, 0 \rightarrow \mathbf{x}, t\right)$. For a deterministic system, with evolution law $\mathbf{x}(t)=S^{t} \mathbf{x}(0)$, the probability of transition reduces to $W\left(\mathbf{x}_{0}, 0 \rightarrow \mathbf{x}, t\right)=\delta\left(\mathbf{x}-S^{t} \mathbf{x}_{0}\right)$, where

$\delta(\cdot)$ is the Dirac delta function. Then we can write an expression for the mean value of the variable $x_{i}$, computed with the density of the perturbed system:

$$
\left\langle x_{i}(t)\right\rangle^{\prime}=\iint x_{i} \rho^{\prime}\left(\mathbf{x}_{0}\right) W\left(\mathbf{x}_{0}, 0 \rightarrow \mathbf{x}, t\right) d \mathbf{x} d \mathbf{x}_{0} .
$$

The mean value of $x_{i}$ during the unperturbed evolution can be written in a similar way:

$$
\left\langle x_{i}(t)\right\rangle=\iint x_{i} \rho\left(\mathbf{x}_{0}\right) W\left(\mathbf{x}_{0}, 0 \rightarrow \mathbf{x}, t\right) d \mathbf{x} d \mathbf{x}_{0} .
$$

Therefore, defining $\overline{\delta x_{i}}=\left\langle x_{i}\right\rangle^{\prime}-\left\langle x_{i}\right\rangle$, we have:

1 The study of an "impulsive" perturbation is not a severe limitation, for instance in the linear regime the (differential) linear response describes the effect of a generic perturbation. 


$$
\begin{aligned}
\overline{\delta x_{i}}(t) & =\iint x_{i} \frac{\rho\left(\mathbf{x}_{0}-\Delta \mathbf{x}_{0}\right)-\rho\left(\mathbf{x}_{0}\right)}{\rho\left(\mathbf{x}_{0}\right)} \rho\left(\mathbf{x}_{0}\right) W\left(\mathbf{x}_{\mathbf{0}}, 0 \rightarrow \mathbf{x}, t\right) d \mathbf{x} d \mathbf{x}_{0} \\
& =\left\langle x_{i}(t) F\left(\mathbf{x}_{0}, \Delta \mathbf{x}_{0}\right)\right\rangle
\end{aligned}
$$

where

$$
F\left(\mathbf{x}_{0}, \Delta \mathbf{x}_{0}\right)=\left[\frac{\rho\left(\mathbf{x}_{0}-\Delta \mathbf{x}_{0}\right)-\rho\left(\mathbf{x}_{0}\right)}{\rho\left(\mathbf{x}_{0}\right)}\right] .
$$

Note that the system is assumed to be mixing, so that the decay to zero of the time-correlation functions prevents any departure from equilibrium.

For an infinitesimal perturbation $\delta \mathbf{x}(0)=\left(\delta x_{1}(0) \cdots \delta x_{N}(0)\right)$, the function in (3.7) can be expanded to first order, if $\rho(\mathbf{x})$ is non-vanishing and differentiable, and one obtains:

$$
\begin{aligned}
\overline{\delta x_{i}}(t) & =-\sum_{j}\left\langle\left. x_{i}(t) \frac{\partial \ln \rho(\mathbf{x})}{\partial x_{j}}\right|_{t=0}\right\rangle \delta x_{j}(0) \\
& \equiv \sum_{j} R_{i, j}(t) \delta x_{j}(0)
\end{aligned}
$$

which defines the linear response

$$
R_{i, j}(t)=-\left\langle\left. x_{i}(t) \frac{\partial \ln \rho(\mathbf{x})}{\partial x_{j}}\right|_{t=0}\right\rangle
$$

of the variable $x_{i}$ with respect to a perturbation of $x_{j}$. One can easily repeat the computation for a generic observable $A(\mathbf{x})$, obtaining:

$$
\overline{\delta A}(t)=-\sum_{j}\left\langle\left. A(\mathbf{x}(t)) \frac{\partial \ln \rho(\mathbf{x})}{\partial x_{j}}\right|_{t=0}\right\rangle \delta x_{j}(0)
$$

In the above derivation of the FDR, we did not use any approximation on the evolution of $\delta \mathbf{x}(t)$. Starting with the exact expression (3.6) for the response, only a linearization of the initial perturbed density is needed, and this implies nothing but the smallness of the initial perturbation. From the evolution of the trajectories difference, one can indeed define the leading Lyapunov exponent $\lambda$, by considering only the positive quantities $|\delta \mathbf{x}(t)|$, so that at small $|\delta \mathbf{x}(0)|$ and large enough $t$ one can write

$$
\langle\ln |\delta \mathbf{x}(t)|\rangle \simeq \ln |\delta \mathbf{x}(0)|+\lambda t
$$

Differently, in the derivation of the FDR, one deals with averages of quantities with sign, such as $\overline{\delta \mathbf{x}(t)}$. This apparently marginal difference is very important 
and underlies the possibility of deriving the FDR without incurring in van Kampen's objection.

At this point one could object that in chaotic deterministic dissipative systems the above machinery cannot be applied, because the invariant measure is not smooth. 2 In chaotic dissipative systems the invariant measure is singular, however the previous derivation of the FDR is still valid if one considers perturbations along the expanding directions. In addition, one is often interested in some specific variables, so that a projection is performed, making irrelevant the singular character of the invariant measure. For a mathematically oriented presentation of these ideas, related to a class of particularly well behaved dynamical systems, see [38]. In these cases, a general response function has two contributions, corresponding respectively to the expanding (unstable) and the contracting (stable) directions of the dynamics. The first contribution can be associated to some correlation function of the dynamics on the attractor (i.e. the unperturbed system). This is not true for the second contribution which is very difficult to extract numerically [39]. Nevertheless, a small amount of noise, that is always present in a physical system, smoothens the $\rho(\mathbf{x})$ and the FDR can be derived. We recall that this "beneficial" noise has the important role of selecting the natural measure, and, in the numerical experiments, it is provided by the round-off errors of the computer. Then, although the introduction of noise in a deterministic framework may look unjustified, the assumption on the smoothness of the invariant measure along the unstable manifold still allows us to avoid subtle technical difficulties, as confirmed by Ruelle's work [38].

We further observe that these dynamical mechanisms, which work equally well in low and high dimensional systems, do not need to constitute the main reason for which $\rho$ can be assumed to be smooth, in systems of physical interest. Indeed, in systems of many particles, the projections from high dimensional phase spaces to a low dimensional space, is the main reason for dealing with smooth distributions.

In Hamiltonian systems, taking the canonical ensemble as the equilibrium distribution, one has $\ln \rho=-\beta H(\mathbf{Q}, \mathbf{P})+$ constant. If $x_{i}$ denotes the component $q_{k}$ of the position vector $\mathbf{Q}$ and $x_{j}$ the corresponding component $p_{k}$ of the momentum $\mathbf{P}$, Hamilton's equations $\left(d q_{k} / d t=\partial H / \partial p_{k}\right)$ lead to the usual FDR $[27,28]$

$$
\frac{\overline{\delta q_{k}}(t)}{\delta p_{k}(0)}=\beta\left\langle\left. q_{k}(t) \frac{d q_{k}}{d t}\right|_{t=0}\right\rangle=-\beta \frac{d}{d t}\left\langle q_{k}(t) q_{k}(0)\right\rangle .
$$

In non Hamiltonian systems, where usually the shape of $\rho(\mathbf{x})$ is not known,

$\overline{2}$ Typically the invariant measure of a chaotic attractor has a multifractal character and its Renyi dimensions $d_{q}$ do not depend on $q[37]$. 
relation (3.9) does not give very detailed information. However it shows the existence of a connection between the mean response function $R_{i, j}$ and a suitable correlation function, computed in the non perturbed systems:

$$
\left\langle x_{i}(t) f_{j}(\mathbf{x}(0))\right\rangle, \quad \text { with } \quad f_{j}(\mathbf{x})=-\frac{\partial \ln \rho}{\partial x_{j}},
$$

where, in general, the function $f_{j}$ is unknown. Let us stress that in spite of the technical difficulty for the determination of the function $f_{j}$, which depends on the invariant measure, a FDR always holds in mixing systems, if the invariant measure can be considered "smooth enough". We note that the nature of the statistical steady state (either equilibrium, or non equilibrium) plays no role at all for the validity of the FDR.

In the case of a multivariate Gaussian distribution, $\ln \rho(\mathbf{x})=-\frac{1}{2} \sum_{i, j} \alpha_{i, j} x_{i} x_{j}+$ const. where $\left\{\alpha_{i, j}\right\}$ is a positive matrix, and the elements of the linear response matrix can be written in terms of the usual correlation functions:

$$
R_{i, j}(t)=\sum_{k} \alpha_{i, k}\left\langle x_{i}(t) x_{k}(0)\right\rangle .
$$

An important nontrivial class of systems with a Gaussian invariant measure is the inviscid hydrodynamics, where the Liouville theorem holds, and a quadratic invariant exists, see Section 4.1 .

\subsection{FDR, chaos and molecular dynamics}

It is not necessary to stress further the relevance of the FDR for the numerical investigation of the transport properties: via a molecular dynamics simulation one can compute the equilibrium correlation functions and therefore, using the Green- Kubo formula, the transport coefficients of liquids models without the introduction of any simplifying assumption. This approach has been successfully used in seminal works $[40,41,17]$.

Another method which is more fundamental, from a conceptual point of view, but rather simple is to use directly the definitions of transport coefficients: one perturbs the system with an external force or imposes driving boundary conditions (e.g. a shear) $[42,43,44,45]$ and observes the relaxation process. For such a problem, van Kampen's objection is relevant, to some extent. For example in numerical simulations, $R_{i, i}(t)$ is obtained by perturbing the variable $x_{i}$ at time $t=t_{0}$ with a small perturbation of amplitude $\delta x_{i}(0)$ and then evaluating the separation $\delta x_{i}\left(t \mid t_{0}\right)$ between the two trajectories $\mathbf{x}(t)$ and $\mathbf{x}^{\prime}(t)$ which are integrated up to a prescribed time $t_{1}=t_{0}+\Delta t$. At time $t=t_{1}$ the variable $x_{i}$ of the reference trajectory is again perturbed with the same $\delta x_{i}(0)$, and a new sample $\delta \mathbf{x}\left(t \mid t_{1}\right)$ is computed and so forth. The procedure is 
repeated $M \gg 1$ times and the mean response is then evaluated:

$$
R_{i, i}(\tau)=\frac{1}{M} \sum_{k=1}^{M} \frac{\delta x_{i}\left(t_{k}+\tau \mid t_{k}\right)}{\delta x_{i}(0)}
$$

In the presence of chaos, the two trajectories $\mathbf{x}(t)$ and $\mathbf{x}^{\prime}(t)$ typically separate exponentially in time, therefore the mean response is the result of a delicate balance of terms which grow in time in different directions. A naive estimate of the error in the computation of $R_{i, i}(\tau)$ suggests an increase in time as

$$
e_{M}(\tau)=\left[\frac{1}{M} \sum_{k=1}^{M}\left(\frac{\delta x_{i}\left(t_{k}+\tau \mid t_{k}\right)}{\delta x_{i}(0)}\right)^{2}-\left(R_{i, i}(\tau)\right)^{2}\right]^{1 / 2} \sim \frac{e^{\frac{L(2)}{2} \tau}}{\sqrt{M}}
$$

where $L(2)$ is the generalized Lyapunov exponent of order 2 :

$$
L(2)=\lim _{\tau \rightarrow \infty} \frac{1}{\tau} \ln \left\langle\left(\frac{|\delta \mathbf{x}(\tau)|}{|\delta \mathbf{x}(0)|}\right)^{2}\right\rangle \text {. }
$$

In the above equation $\delta \mathbf{x}(\tau)$ is assumed infinitesimal, i.e. evolving according the linearized dynamics (in mathematical terms, $\delta \mathbf{x}(\tau)$ is a tangent vector of the system) [46], and a lower bound for $L(2)$ is given by $2 \lambda$. Thus very large $M$ seems to be necessary, in order to properly estimate this balance and to compute $R_{i, i}(\tau)$ for large $\tau$.

On the other hand, the numerical results obtained with molecular dynamics simulations show rather clearly that the presence of chaos does not introduce severe limitations in the range of validity of the FDR, in agreement with the intuition of Kubo and the derivation of the FDR in the previous section. For instance in $[42,43]$, for an ion in liquid argon, a perturbing force of order $10^{5} \mathrm{eV} / \mathrm{cm}$ has been used, and a rather good agreement has been obtained between the results produced perturbing the system and those deduced from the Green-Kubo formula. These results are not restricted to systems with a large number of degrees of freedom [47,32]. As a final technical remark we note that the error estimate given by (3.16) is rather conservative and the uncertainty is actually smaller (at least for $\tau$ not too large). In [47] one can see that for $\tau<\mathcal{T}$ (where $\mathcal{T}$ is order of the correlation time) $e_{M}(\tau)$ first decreases and later increases exponentially, but with a rate different from $L(2)$. Indeed it is not completely correct in this context to use at finite time the Lyapunov exponents (or analogous quantities), which are well defined only at very large times. 3

3 The same subtle problem appears in the connection between the KolmogorovSinai entropy, $h_{K S}$ (which is linked to the Lyapunov exponents via the Pesin formula[48]), and the variation rate of the coarse grained Gibbs entropy, $r_{G}$. Detailed numerical computations show that typically the (often accepted) identification of the two quantities does not hold. The basic reason of this fact is in the asymptotic 


\subsection{Linear response theory, foundations of statistical mechanics and anoma- lous diffusion}

This section is devoted to a brief discussion of interesting, and still partly open issues involving the relation among linear response theory, the foundations of statistical mechanics and systems with anomalous diffusion.

\subsubsection{Linear response theory and the foundations of statistical mechanics}

In his celebrated book Mathematical Foundations of Statistical Mechanics, Khinchin obtained important results concerning the ergodic and mixing problem in statistical mechanics, without recourse to the notion of metrical transitivity, required by the Birkhoff theorem [50]. The general idea of this approach, which will be considered also in Chapter 5, is that the systems of interest in statistical mechanics have a very large number of degrees of freedom and the important observables are not generic functions.

In a system with many degrees of freedom and weak interactions among the particles 4 , consider an observable $A$ which contains the coordinates of only one (or a few) particles. Khinchin has shown that the ergodic hypothesis for a quantity $A$, i.e. the validity of

$$
\lim _{T \rightarrow \infty} \frac{1}{T} \int_{0}^{\infty} A(t) d t=\langle A\rangle,
$$

holds if the auto-correlation of $A$ satisfies the relation

$$
\lim _{t \rightarrow \infty}\langle A(t) A(0)\rangle=\langle A\rangle^{2} .
$$

This result can be reversed, i.e. the auto-correlation $C_{A}(t)=\langle A(t) A(0)\rangle$ satisfies (3.19) if Eq. (3.18) holds uniformly [52]5.

(with respect to time) nature of $h_{K S}$, while $r_{G}$ is a quantity related to the short time behavior of a given system [49].

4 Khinchin considers a separable Hamiltonian system i.e.:

$$
H=\sum_{n=1}^{N} H_{n}\left(\mathbf{q}_{n}, \mathbf{p}_{n}\right) .
$$

It is reasonable to think that the result holds also in systems of particles interacting through a short range potential, as those studied in [51] for equilibrium statistical mechanics.

5 Note that this result concerns the ergodic hypothesis for only the given observable $A$, which is much weaker than ergodicity in its strict technical meaning, indeed it holds only for specific observables, and in systems with many degrees of freedom. 
On the other hand, we know that correlation functions are closely related to the response function, so it is quite natural to investigate the connection between ergodicity and linear response theory. In a series of papers $[53,54,55,56,57]$ this problem has been discussed in detail. The main result is the possibility to connect ergodic or non ergodic behaviour to the response of a system to an external perturbation. One finds that, in a generic system, irreversibility, i.e. the validity of (3.19), is a necessary but not sufficient condition for ergodicity.

Let us indicate, in a symbolic way, the variables which determine the macroscopic state and the control parameters with $\{A\}$ and $\{P\}$, respectively. If we perturb the system changing $\{P\}$ at time $t=0$, i.e. $\{P\} \rightarrow\{P\}+\{\delta P\} \Theta(t)$ one has

$$
\overline{\delta A(t)}=\delta P \int_{0}^{t} R\left(t^{\prime}\right) d t^{\prime}
$$

where $\{R\}$ indicates the (differential) response function. For the sake of simplicity, we assume $\langle A\rangle=0$. If ergodicity holds for the variable $A$, one has $[53,54]$ :

$$
\lim _{T \rightarrow \infty} \frac{1}{T} \int_{0}^{T} \int_{0}^{t} R_{A}\left(t-t^{\prime}\right) d t d t^{\prime}=\chi_{A}
$$

where $\chi_{A}$ is the static susceptibility. The advantage of (3.21) over the usual condition for ergodicity, Eq. (3.18), is that both sides of (3.21) may be evaluated, for specific models, knowing the unperturbed measure (or equivalently the Hamiltonian). In the linear response theory, for Hamiltonian systems, one has

$$
R_{A}(t)=-\frac{d}{d t} C_{A}(t)=-\frac{d}{d t}\langle A(t) A(0)\rangle .
$$

Denoting with $\tilde{R}_{A}(z)$ and $\tilde{C}_{A}(z)$ the Laplace transform of $R_{A}(t)$ and $C_{A}(t)$, respectively, from (3.21-3.22), and using the condition (valid for differentiable variables) $d C_{A}(t) / d t=0$ for $t=0$, the limit $T \rightarrow \infty$ produces

$$
\tilde{R}_{A}(0)+C_{A}(T)-\frac{\tilde{C}_{A}(0)}{T} \rightarrow \chi_{A}
$$

or in different form:

$$
\tilde{R}_{A}(0)+\left.z \tilde{C}_{A}(z)\right|_{z=0}=\chi_{A} .
$$

In general one does not know if (3.21), or its equivalent (3.24), holds. It is possible to show that the two equations hold and coincide if $C_{A}(t)$ approaches zero for large $t$ in a fast way, so that

$$
\int_{0}^{\infty} C_{A}(t) d t<\infty
$$

In other words, the condition that $C_{A}(t) \rightarrow 0$ for $t \rightarrow \infty$ is only necessary for ergodicity, while (3.25) is sufficient. Note that (3.25) is implied by the GreenKubo formulae, when the $\{\chi\}$ are finite quantities. The Khinchin theorem, on the other side, requires weak interactions among the components of the 
system: this is no more guaranteed, in the general case, when $C_{A}(t)$ can have a slow decay.

In $[53,54,55]$ one can find a detailed discussion on different systems (including monoatomic chains, oscillator models, chains with impurity, etc.) where the powerful recurrence relations method can be successfully applied and it is possible to determine whether the condition (3.25) is valid or not. Consider, for instance, the case of a nearest-neighbor coupled harmonic oscillator chain of $2 N+1$ atoms, with periodic boundary conditions, containing a tagged particle of mass $M$, while the remaining have mass $m$. In the $N \rightarrow \infty$ limit, it is possible to see that the validity of (3.25) depends on the value of $\lambda=m / M$. If $\lambda$ is finite, the momentum of the tagged particle is an ergodic observable.

\subsubsection{The fluctuation- dissipation relation in the case of anomalous diffusion}

In recent years, many authors have shown that one can have anomalous diffusion in certain systems $[58,59]$, i.e. one can have

$$
\left\langle x^{2}(t)\right\rangle \sim t^{2 \nu} \text { with } \nu \neq 1 / 2
$$

Formally this corresponds to $D=\infty$ if $\nu>1 / 2$ (superdiffusion) and $D=0$ if $\nu<1 / 2$ (subdiffusion).

Here we shall not discuss the different mechanisms at the origin of anomalous diffusion [59]. Our aim is to provide a short discussion of the following problem, which comes out in a rather natural way in presence of anomalous diffusion. We know, from Chapter 2, that, if in an unperturbed system $\langle x(t)\rangle=0$, in the cases with standard diffusion, i.e. with a finite positive diffusion coefficient, the response to a small external force is a linear drift

$$
\overline{x(t)} \sim t
$$

where the over-bar indicates the average over the perturbed system. One may ask how this scenario changes in presence of anomalous diffusion.

As a consequence of the simple identity

$$
\left\langle x^{2}(t)\right\rangle=\int_{0}^{t} \int_{0}^{t} C_{v}\left(t_{1}-t_{2}\right) d t_{1} d t_{2} \quad, \quad C_{v}\left(t_{1}-t_{2}\right)=\left\langle v\left(t_{1}\right) v\left(t_{2}\right)\right\rangle
$$

and the fluctuation dissipation relation, one has the formal relation

$$
\frac{d}{d t} \overline{x(t)}=K \int_{0}^{t} C_{v}\left(t^{\prime}\right) d t^{\prime}
$$

where $K$ is a constant depending on the physical details, whose value is not 
relevant for our purpose. In the superdiffusive case, one has

$$
C_{v}(t) \sim t^{-\alpha} \text { with } \alpha<1
$$

and $\nu=1-\alpha / 2$.

A straightforward consequence of (3.29) seems to be

$$
\overline{x(t)} \sim t^{2 \nu}
$$

On the other hand it is not difficult to realize that such a formal argument is not reliable. Let us consider again the previous discussion, i.e. take a system whose state is $\{A\}$ and the control parameters are $\{P\}$. Consider a special value $\left\{P^{*}\right\}$ of $\{P\}$ such that (3.25) does not hold. Now perturb the system changing $\{P\}$ at time $t=0$, so that $\left\{P^{*}\right\} \rightarrow\left\{P^{*}\right\}+\{\delta P\} \Theta(t)$. In our case $R_{v}(t)=C_{v}(t)$, taking $t \rightarrow \infty$ in $(3.20)$, we have $\overline{\delta A(\infty)}=\infty$, if $\nu>1 / 2$. The validity of such a result is highly questionable: for instance it is easy to obtain a finite result:

$$
\overline{\delta A(\infty)}=\langle A\rangle_{P^{*}+\delta P}-\langle A\rangle_{P^{*}}
$$

Let us discuss separately the subdiffusive case $(\nu<1 / 2)$ and the superdiffusive case $(\nu>1 / 2)$.

3.4.2.1 The subdiffusive case Some authors show that in the subdiffusive case, the result (3.31) holds [60]. This has been explicitly proved in systems described by a fractional-Fokker-Planck equation. In the case of an overdamped particle, see Sect. 2.3.4, the usual Fokker-Planck equation is

$$
\frac{\partial}{\partial t} P(x, t)=\mathcal{L}_{F P} P(x, t)=\frac{\partial}{\partial x}\left(\frac{V^{\prime}(x)}{m \eta_{1}} P(x, t)\right)+K_{1} \frac{\partial^{2}}{\partial x^{2}} P(x, t),
$$

with $K_{1}=k_{B} T / m \eta_{1}$. For $V=0$ one has

$$
\left\langle x^{2}(t)\right\rangle \simeq 2 K_{1} t
$$

while for $V=-F x$,

$$
\overline{x(t)} \simeq \frac{F}{m \eta_{1}} t
$$

which is nothing but the Einstein relation for such a system. The fractionalFokker-Planck equation is given by

$$
\frac{\partial}{\partial t} P(x, t)=\mathcal{D}_{T}^{1-\gamma}\left[\mathcal{L}_{F P} P(x, t)\right]
$$


where now $\mathcal{L}_{F P}$ is defined as in (3.33) with the changes $\eta_{1} \rightarrow \eta_{\gamma}$ and $K_{1} \rightarrow$ $K_{\gamma}$, and $\mathcal{D}_{T}^{1-\gamma}$ is the so-called Riemann-Liouville fractional operator defined through:

$$
\mathcal{D}_{T}^{1-\gamma}[f(x, t)]=\frac{1}{\Gamma(\gamma)} \frac{\partial}{\partial t} \int_{0}^{t} \frac{f\left(x, t^{\prime}\right)}{\left(t-t^{\prime}\right)^{1-\gamma}} d t^{\prime} \text { with } 0<\gamma \leq 1
$$

being $\Gamma(\gamma)$ the Euler gamma function. For $V=0$ one has a generalization of $(3.34)$ :

$$
\left\langle x^{2}(t)\right\rangle \simeq 2 \frac{K_{1} t^{\gamma}}{\Gamma(1+\gamma)}
$$

and for $V=-F x$,

$$
\overline{x(t)} \simeq \frac{F}{m \eta_{\gamma}} \frac{t^{\gamma}}{\Gamma(1+\gamma)},
$$

in other words, if we assume the relation $K_{\gamma}=k_{B} T / m \eta_{\gamma}$, one has:

$$
\overline{x(t)}=\frac{1}{2} \frac{F\left\langle x^{2}(t)\right\rangle}{k_{B} T}
$$

which generalizes the Einstein relation for the subdiffusive case. The investigation of charge carrier transport in semiconductors [61] showed that, up to a prefactor, which could not be determined exactly, eq. (3.40) is indeed valid.

3.4.2.2 The superdiffusive case This situation is much more delicate, and, as far as we know, the problem is still open. The difficulties are due to the fact that the mechanism for the anomalous diffusion is rather subtle and can be obtained only in systems with rather peculiar dynamics. For instance in deterministic chaotic systems with a fine tuning of the parameters [58,62]. Typically the following scenario holds: if for a certain value $P_{0}=P^{*}$ of the control parameters one has $C_{v}(t) \sim t^{-\alpha}$ with $\alpha<1$ and therefore $\nu>1 / 2$, a small perturbation $P_{0} \rightarrow P_{p}=P^{*}+\delta P$ restores the standard diffusion with $\nu=1 / 2$. More complex is the case of non-chaotic systems, which also show anomalous diffusion [63], but it is not completely clear which perturbations restore normal diffusion.

At first, the difference between $P^{*}$ and $P^{*}+\delta P$ can appear a paradox, on the other hand a precise analysis of the problem shows that the anomalous behaviour for $P=P_{0}=P^{*}$ induces a sort of "ghost" for $P=P_{p}=P^{*}+\delta P$, which is reminiscent of the unperturbed anomalous behavior. For $P=P_{p}$ the correlation function $C_{v}(t)$ is integrable, however for $t<t_{c}(\delta P)$ (where $t_{c}(\delta P) \rightarrow \infty$ as $\left.\delta P \rightarrow 0\right)$ one has $C_{v}(t) \sim t^{-\alpha}$, therefore the difference between the anomalous case (for $P_{0}$ ) and the standard diffusion (for $P_{p}$ ) appears only at very large time, leading to a large diffusion coefficient (diverging for $\delta P \rightarrow$ 0) $[64]$. 
With the above scenario one can try to give a solution to the problem. Let us denote by $C_{v}^{(0)}(t)$ and $C_{v}^{(p)}(t)$ the correlation function for $P_{0}=P^{*}$ and $P_{p}=P^{*}+\delta P$ respectively. In the presence of anomalous behavior (i.e. when (3.25) is not valid) it is not possible to exchange the limits $\delta P \rightarrow 0$ and $t \rightarrow \infty$. Since the relaxation time of the perturbed system is $t_{c}$, Eq. (3.29) is valid only for $t<t_{c}$, while for $t>t_{c}$, Eq. (3.29) must be replaced by:

$$
\frac{d}{d t} \overline{x(t)}=K \int_{0}^{t_{c}} C_{v}^{(0)}\left(t^{\prime}\right) d t^{\prime}+K \int_{t_{c}}^{t} C_{v}^{(p)}\left(t^{\prime}\right) d t^{\prime}
$$

As a consequence Eq. (3.31) holds in the range $t<t_{c}$, while for larger times one has the standard result (3.27), with a different prefactor depending on $t_{c}$. One can say that also in anomalous diffusion, a "ghost" for $P^{*}$ remains up to large but finite times $t_{c}$.

A detailed discussion of this problem for a dynamical model has been given in [65]. Both possibilities, that $\delta P \rightarrow 0$ before $t \rightarrow \infty$, and that $t \rightarrow \infty$ before $\delta P \rightarrow 0$, have been considered. In the first case, one obtains $\overline{x(t)} \sim t^{2 \nu}$, for the response at large times; on the other hand, for any finite value of $\delta P$, $\overline{d x(t)} / d t$ saturates to a finite value, that depends on the perturbation. The general scenario suggested in Eq. (3.41) is therefore verified.

\section{Some applications}

The FDR has been extensively used in equilibrium statistical mechanics. Applications in near-equilibrium contexts are discussed in any advanced book dealing with transport phenomena. In this section we want to discuss how to use the FDR in less standard cases, namely non-equilibrium and nonHamiltonian systems.

\subsection{Fluid dynamics}

The Fluctuation-Response theory was originally developed within the framework of equilibrium statistical mechanics of Hamiltonian systems, giving rise

to some confusion and misleading ideas about its validity. For instance, some authors claimed that in fully developed turbulence (which is a non Hamiltonian and non equilibrium system) there is no relation between spontaneous fluctuations and relaxation to the statistical steady state [66].

The confusion is due, at least in part, to terminology; for instance, at times the term FDR is used just for Eq. (3.14) which is valid in the Gaussian case. Nevertheless the FDR plays a role in analytical approaches to the statistical description of hydrodynamics, where Green functions are naturally involved, 
both in perturbative theories and in closure schemes $[67,68,69]$, as well as in other fields, like in geophysical or climate studies[70,71,72].

\subsubsection{Interlude on Statistical Mechanics of Fluids}

The Navier-Stokes equations for the evolution of the velocity field $\mathbf{v}(\mathbf{x}, t)$ of an incompressible fluid are:

$$
\begin{gathered}
\frac{\partial \mathbf{v}(\mathbf{x}, t)}{\partial t}+(\mathbf{v}(\mathbf{x}, t) \cdot \nabla) \mathbf{v}(\mathbf{x}, t)=-\frac{1}{\rho} \nabla p(\mathbf{x}, t)+\nu \Delta \mathbf{v}(\mathbf{x}, t)+\mathbf{F}(\mathbf{x}, t), \\
\nabla \cdot \mathbf{v}(\mathbf{x}, t)=0,
\end{gathered}
$$

where $p(\mathbf{x}, t)$ is the pressure in the point $\mathbf{x}$ at time $t ; \rho$ and $\nu$ are the density and the kinematic viscosity of the fluid respectively.

For a perfect fluid (i.e. $\nu=0)$ and in the absence of external forces $(\mathbf{F}=0)$, the evolution of the velocity field is given by the Euler equations, which conserve the kinetic energy. Such a case may be easily treated within an equilibrium statistical mechanics framework, simply following the approach used for the Hamiltonian statistical mechanics [73]. Consider a fluid in a cube of edge $L$ and assume periodic boundary conditions, so that the velocity field can be expressed in terms of Fourier series as follows:

$$
\mathbf{v}(\mathbf{x}, t)=\frac{1}{L^{d / 2}} \sum_{\mathbf{k}} \mathbf{u}(\mathbf{k}, t) e^{i \mathbf{k x}}
$$

with $\mathbf{k}=2 \pi \mathbf{n} / L$ and $\mathbf{n}=\left(n_{1}, \ldots, n_{d}\right), n_{j}$ being integers and $d$ the spatial dimension. By introducing (4.3) in the Euler equations, and imposing an ultraviolet cutoff, $\mathbf{u}(\mathbf{k})=0$ for $k>K_{\max }$, one obtains a set of ordinary differential equations of the form:

$$
\frac{d Y_{a}}{d t}=\sum_{b, c}^{N} A_{a b c} Y_{b} Y_{c}
$$

where $N \sim K_{\max }^{d}$ is the number of degrees of freedom, and the variables $\left\{Y_{a}\right\}$ are a subset of the full Fourier spectrum $\{\mathbf{u}(\mathbf{k})\}$. The incompressibility condition yields $\mathbf{k} \cdot \mathbf{u}(\mathbf{k})=0$, and the fact that $\mathbf{u}(\mathbf{k})$ is real yields $\mathbf{u}(\mathbf{k})=$ $\mathbf{u}^{*}(-\mathbf{k})$.

As a consequence of the energy conservation one has

$$
\frac{1}{2} \sum_{a} Y_{a}^{2}=E=\text { constant }
$$

In addition, the coefficients $A_{a b c}$ have the properties $A_{a b c}=A_{a c b}, A_{a b c}+$ $A_{b c a}+A_{c a b}=0$, and incompressibility makes the Liouville theorem hold:

$$
\sum_{a} \frac{\partial}{\partial Y_{a}} \frac{d Y_{a}}{d t}=0
$$


so that the necessary ingredients for an equilibrium statistical mechanical approach are all present. Assuming, in analogy with the treatment of Hamiltonian systems, a microcanonical distribution on the constant energy surface $\frac{1}{2} \sum_{a} Y_{a}^{2}=E$, for large $N$ it is easy to obtain the invariant probability density of $Y_{a}$ :

$$
P_{i n v}\left(\left\{Y_{a}\right\}\right) \propto \exp \left(-\frac{\beta}{2} \sum_{a} Y_{a}^{2}\right)
$$

and the energy equipartition $\left.<Y_{a}^{2}\right\rangle=2 \frac{E}{N}=\beta^{-1}$.

In two dimensions another quantity is conserved: the enstrophy $\frac{1}{2} \int \mid \nabla \times$ $\left.\mathbf{u}(\mathbf{x}, t)\right|^{2} d \mathbf{x}$ which, in terms of the $\left\{Y_{a}\right\}$, can be written as

$$
\frac{1}{2} \sum_{a} k_{a}^{2} Y_{a}^{2}=\Omega=\text { const }
$$

Assuming a microcanonical distribution on the surface where both the energy and the enstrophy are constant, and taking the large $N$ limit, one obtains

$$
P_{i n v}\left(\left\{Y_{a}\right\}\right) \propto \exp -\frac{1}{2}\left(\beta_{1} \sum_{a} Y_{a}^{2}+\beta_{2} \sum_{a} k_{a}^{2} Y_{a}^{2}\right)
$$

where the Lagrange multipliers $\beta_{1}$ and $\beta_{2}$ are determined by $E$ and $\Omega$.

Other systems, like magnetohydrodynamics and geostrophic fluids, have quadratic invariants and obey the Liouville theorem in the inviscid limit. 6 In such situations it is straightforward to generalize the previous results as

$$
P_{i n v}\left(\left\{Y_{a}\right\}\right) \propto \exp \left(-\frac{1}{2} \sum_{a, b} \alpha_{a, b} Y_{a} Y_{b}\right)
$$

where $\left\{\alpha_{a, b}\right\}$ is a positive matrix dependent on the specific form of the invariants and on the values of the Lagrange multipliers.

A statistical steady state requires a forcing in the case of a viscous fluid, which results in:

$$
\left(\frac{d}{d t}+\nu k_{a}^{2}\right) Y_{a}=\sum_{b, c}^{N} A_{a b c} Y_{b} Y_{c}+f_{a},
$$

instead of eq. (4.4). It is well known[69] that in $3 D$ the limit $\nu \rightarrow 0$ is singular and cannot be interchanged with the limit $K_{\max } \rightarrow \infty$, therefore the statistical

6 Numerical results in $2 D$ and $3 D$ inviscid hydrodynamics, as well as in other inviscid cases (e. g. magnetohydrodynamics) show that the systems described by the inviscid truncated Eq. (4.4) with quadratic invariant, are ergodic and mixing[74,75]: arbitrary initial distributions of $\left\{Y_{a}\right\}$ evolve towards the multivariate Gaussian (4.10). 
mechanics of an inviscid fluid has a rather limited relevance for the NavierStokes equations at very high Reynolds numbers. 7 In the $\nu \rightarrow 0$ limit in presence of forcing at large scale (small $k$ ) one has an intermediate range, called the inertial range, which has neither pumping nor dissipation and shows a strong departure from equipartition[69,73]. Instead of $\left\langle Y_{a}^{2}\right\rangle=$ const. one has $\left\langle Y_{a}^{2}>\sim k_{a}^{-\gamma}\right.$ where $\gamma \simeq 11 / 3$, the value $\gamma=11 / 3$ corresponding to the Kolmogorov spectrum $8(k) \sim k^{-5 / 3}$.

\subsubsection{A general remark on the decay of correlation functions}

Using general arguments, one could naively conclude that all typical correlation functions, at large time delay, have to relax to zero with the same characteristic time related to spectral properties of the operator $\mathcal{L}$, governing the time evolution of the probability distribution $P(\{Y\}, t)$ :

$$
\frac{\partial}{\partial t} P(\{Y\}, t)=\mathcal{L} P(\{Y\}, t) .
$$

The argument goes as follows: let the time evolution of the system be described by ordinary differential equations $d Y_{a} / d t=X_{a}(\{Y\})$ so that $\mathcal{L} P(\{Y\}, t)=$

$-\sum_{a} \frac{\partial}{\partial Y_{a}}\left(X_{a}(\{Y\}) P(\{Y\}, t)\right)$, for the system (4.11), where $X_{a}=\sum_{a, b} A_{a b c} Y_{b} Y_{b}-$ $\nu k_{a}^{2} Y_{a}+f_{a} \cdot 9$ Let us introduce the eigenvalues $\left\{\alpha_{k}\right\}$ and the eigenfunctions $\left\{\psi_{k}\right\}$ of $\mathcal{L}:$

$$
\mathcal{L} \psi_{k}=\alpha_{k} \psi_{k} .
$$

Of course $\psi_{0}=P_{\text {inv }}$ and $\alpha_{0}=0$; moreover the mixing condition requires that $\operatorname{Re} \alpha_{k}<0$ for $k=1,2, \ldots$. Furthermore assuming that the coefficients $\left\{g_{1}, g_{2}, \ldots\right\}$ and $\left\{h_{1}, h_{2}, \ldots\right\}$ exist, such that the expansion of the functions $g(\{Y\})$ and $h(\{Y\})$ are unique:

$$
g(\{Y\})=\sum_{k=0} g_{k} \psi_{k}(\{Y\}), h(\{Y\})=\sum_{k=0} h_{k} \psi_{k}(\{Y\}),
$$

$\overline{7}$ In $2 D$ the use of conservative statistical mechanics has been proposed to justify some behaviors of real fluids, e.g. Jupiter's red spot and the formation of coherent structures [76,77]. This approach, although interesting, is limited to some specific two-dimensional situations.

$8 E(k) d k$ is the contribution to the kinetic energy of the wave numbers in the range $[k, k+d k]$.

${ }^{9}$ In the presence of white noise terms $\left\{\eta_{a}\right\}$ in eq. (4.11), i.e. $X_{a} \rightarrow X_{a}+\eta_{a}$ where $\left\{\eta_{a}\right\}$ are Gaussian processes with $\left\langle\eta_{a}(t)>=0\right.$ and $\left\langle\eta_{a}(t) \eta_{b}\left(t^{\prime}\right)>=2 \Lambda_{a b} \delta\left(t-t^{\prime}\right)\right.$, one has to add to $\mathcal{L}$ the term[13]

$$
\sum_{a b} \Lambda_{a b} \frac{\partial^{2}}{\partial Y_{a} \partial Y_{b}} .
$$


one has

$$
C_{g, f}(t)=\sum_{k=1} g_{k} h_{k}<\psi_{k}^{2}>e^{\alpha_{k} t},
$$

where $C_{g, f}(t)=<g(\{Y(t)\}) h(\{Y(0)\})>-<g(\{Y\})><h(\{Y\})>$. For "generic", i.e. not orthogonal to $\psi_{1}$, so that $g_{1} \neq 0$ and $h_{1} \neq 0$, functions $g$ and $f$, the quantity $C_{g, f}(t)$ approaches zero at large time:

$$
C_{g, f}(t) \sim e^{-t / \tau_{c}}, \tau_{c}=\frac{1}{\left|\operatorname{Re} \alpha_{1}\right|} .
$$

Naively this argument seems to imply that in hydrodynamic systems all the correlation functions, $C_{a, a}(t)=<Y_{a}(t) Y_{a}(0)>$, relax to zero with the same characteristic time for all $k_{a}$, while one expects a whole hierarchy of characteristic times distinguishing the behavior of the correlation functions at different scales [69]. The paradox is, of course, only apparent since the above argument is valid only at very long times, i.e. much longer than the longest characteristic time, hence it tells nothing about systems with many different time scales.

\subsubsection{FDR in perfect fluids and in turbulence}

Although the inviscid fluid mechanics is not a Hamiltonian system, Eqs. (3.3) and (4.7) (or (4.9) in 2D) make the FDR take the simple form

$$
R_{a, b}=\frac{C_{a, a}(t)}{C_{a, a}(0)} \delta_{a b}
$$

because of the Gaussian statistics. To the best of our knowledge, Kraichnan was the first to realize that the FDR may hold in a non Hamiltonian context $[33,34]$. As a matter of fact, in the case of turbulence the scenario is not too simple for two reasons:

a) the (unknown) invariant measure is not Gaussian

b) the degrees of freedom $\left\{Y_{a}\right\}$ are not independent.

Nevertheless the general result discussed in Section 3 suggests that a relation between response and a suitable correlation function must exist. Indeed, also in turbulence there are many degrees of freedom, which are likely to yield a smooth distribution, when projecting on lower dimensional spaces, as required for the FDR.

The FDR plays an important role in the statistical closure problem, which has to be tackled by any non linear theory. In our case the evolution equations for $C_{a a}(t)=<Y_{a}(t) Y_{a}(0)>$ have the form

$$
\left(\frac{d}{d t}+\nu k_{a}^{2}\right) C_{a a}=F_{a}\left(C^{(3)}\right)
$$

where $F_{a}$ is a function of the third-order correlations $C^{(3)}$ i.e. of terms like $\left\langle Y_{a}(t) Y_{b}(0) Y_{c}(0)\right\rangle$. In the equations for the $C^{(3)}$, fourth-order correlations $C^{(4)}$ 
appear, and so on. An analogous situation occurs in the kinetic theory of gases, with the Bogolyubov-Born-Green-Kirkwood-Yvon hierarchy[78]. Experience has shown that it is quite efficient to truncate the hierarchy at a certain level, making some closure hypothesis. The simplest assumption in our case is the quasi-normal approximation, which assumes that the fourth-order cumulants vanish, i.e that

$$
\begin{aligned}
<Y_{a} Y_{b} Y_{c} Y_{d}>=< & Y_{a} Y_{b}><Y_{c} Y_{d}> \\
& +<Y_{a} Y_{c}><Y_{b} Y_{d}>+<Y_{a} Y_{d}><Y_{b} Y_{c}>
\end{aligned}
$$

Unfortunately the resulting set of equations gives unphysical results, such as negative $E(k)$ for some $k$. Therefore it is necessary to introduce more precise hypotheses. One approximation which has been successfully used is the so called eddy-damped quasi-normal Markovian approximation, which takes into account the physical mechanism of the energy transfer[79].

An alternative approach is the direct-interaction-approximation (DIA) developed by Kraichnan[67,80] which is one of the few self-consistent analytical turbulence theory so far discovered. In the DIA one writes separately equations for responses $\{R\}$ and for correlations $\{C\}$

$$
\begin{aligned}
& \left(\frac{\partial}{\partial t}+\nu k_{a}^{2}\right) C_{a, a}\left(t, t^{\prime}\right)=\mathcal{F}_{a}(\{C\},\{R\}) \\
& \left(\frac{\partial}{\partial t}+\nu k_{a}^{2}\right) R_{a, a}\left(t, t^{\prime}\right)=\mathcal{G}_{a}(\{C\},\{R\})
\end{aligned}
$$

where $C_{a, a}\left(t, t^{\prime}\right)=<Y_{a}(t) Y_{a}\left(t^{\prime}\right)>, R_{a, a}\left(t, t^{\prime}\right)=\overline{\delta Y_{a}(t)} / \delta Y_{a}\left(t^{\prime}\right), \mathcal{F}_{a}$ and $\mathcal{G}_{a}$ are (rather complicated) nonlinear integral expressions involving $\{C\}$ and $\{R\}$. The DIA equations give the correct results (including the FDR in the form (4.17)) in the inviscid limit. In addition, for the "random coupling model", i.e.

$$
\left(\frac{d}{d t}+\nu k_{a}^{2}\right) Y_{a}=\sum_{b, c} A_{a b c}^{\prime} Y_{b} Y_{c}
$$

where $A_{a b c}^{\prime}= \pm A_{a b c}$ with the sign varying randomly from triad to triad $(a, b, c)$, Kraichnan[67] showed that DIA equations are exact. 10 Although the DIA equations $(4.20,4.21)$ are closed, they are very complicated, so simplifications are usually introduced, like the so called Simple-Pole Model [82] where basically one assumes the FDR in its simplest form:

$$
C_{a, a}\left(t, t^{\prime}\right)=R_{a, a}\left(t, t^{\prime}\right) C_{a, a}\left(t^{\prime}, t^{\prime}\right) \quad t \geq t^{\prime}
$$

$\overline{10}$ The DIA gives exact results also in other cases, e.g. in the large- $N$ limit of the spherical model of turbulence [81] where the velocity field in (4.1) is now a vector with $N$ components, i.e. $\mathbf{v} \in \mathbf{R}^{N}$, while $\mathbf{x} \in \mathbf{R}^{3}$. 
It has been shown that renormalization group methods and the so called timeordering approach $[83,68]$ give the same results with an exponential form:

$$
\begin{gathered}
C_{a, a}\left(t, t^{\prime}\right)=C_{a, a}\left(t^{\prime}, t^{\prime}\right) e^{-\omega\left(k_{a}\right)\left|t-t^{\prime}\right|} \quad t \geq t^{\prime}, \\
R_{a, a}\left(t, t^{\prime}\right)=e^{-\omega\left(k_{a}\right)\left(t-t^{\prime}\right)} \quad t \geq t^{\prime},
\end{gathered}
$$

with $\omega(k) \sim k^{-2 / 3}$ in agreement with simple dimensional arguments.

Note that Eq. (3.13) indicates that assumption (4.23) is equivalent to assume Gaussian statistics for $\{Y\}$ without imposing a specific covariance matrix. Since it is well known, from both numerical and experimental results, that for very high Reynolds numbers, the statistics of the velocity fields is rather far from Gaussian, e.g. one has long tails in the PdF and intermittent behavior[69,73], eq.(4.23), or eqs. (4.24, 4.25), can not be completely correct. However on intuitive grounds one expects the times characterizing the responses $\{R\}$ to approximate the characteristic correlation times of $\{C\}$. Numerical investigations [80] at moderate Reynolds number of the DIA equations, show that $R_{a, a}\left(t, t^{\prime}\right)$ is not exactly proportional to $C_{a, a}\left(t, t^{\prime}\right)$, however comparing the correlation times $\tau_{C}\left(k_{a}\right)$ (e.g. the time after which the correlation function becomes lower than $1 / 2$ ) and the response time $\tau_{R}\left(k_{a}\right)$ (e.g. the time after which the response function becomes lower than $1 / 2)$, one has that the ratio $\tau_{C}\left(k_{a}\right) / \tau_{R}\left(k_{a}\right)$ stays constant through the inertial range.

\subsubsection{Numerical results for simplified models}

Let us now discuss some numerical tests of the FDR in simplified models of fluid dynamics. Such systems are far from being realistic, but are nontrivial and share the main features of the Navier- Stokes, or the Euler equations. Consider the model [84]:

$$
\frac{d Y_{n}}{d t}=Y_{n+1} Y_{n+2}+Y_{n-1} Y_{n-2}-2 Y_{n+1} Y_{n-1}
$$

with $n=(1,2, \ldots, N)$ and the periodic condition $Y_{n+N}=Y_{n}$. This system, originally introduced as a toy model for chaos in fluid mechanics[84], contains some of the main features of inviscid hydrodynamics: a) quadratic interactions; b) a quadratic invariant, $E=\frac{1}{2} \sum_{n=1}^{N} Y_{n}^{2}$; c) the Liouville theorem holds. For sufficiently large $N$ the distribution of each variable $Y_{n}$ is Gaussian. In this situation, the Gaussian-like FDR holds for each of the $n$ variables, i.e. selfresponse functions to infinitesimal perturbations are indistinguishable from the corresponding self-correlation functions [32].

We can slightly modify the system (4.26) in order to have variables with different characteristic times; for instance, by rescaling the evolution time of 
each variable[85]:

$$
\frac{d Y_{n}}{d t}=k_{n} \cdot\left(Y_{n+1} Y_{n+2}+Y_{n-1} Y_{n-2}-2 Y_{n+1} Y_{n-1}\right)
$$

where the factor $k_{n}$ is defined as $k_{n}=\alpha \cdot \beta^{n}$, with $\beta>1$, for $n=1,2, \ldots, N / 2$, with the "mirror" property $k_{n+N / 2}=k_{N / 2+1-n}$. For the system (4.27) a quadratic integral of motion exists:

$$
I=\sum_{n=1}^{N} \frac{Y_{n}^{2}}{k_{n}}
$$

Moreover, if $N \gg 1$ the $\left\{Y_{n}\right\}$ variables preserve the Gaussian statistics with $<Y_{n} Y_{m}>=<Y_{n}^{2}>\delta_{n m} \propto k_{n}$. As a consequence the simplest FDR, i.e. Eq. (4.17), holds for each of the variables. On the other hand, at variance with the original system (4.26), each $Y_{n}$ has here its own characteristic time. Figure 1 shows the (expected) validity of (4.17), however the shape of $C_{n n}(t)$ changes with $n$. The correlation time of a variable $Y_{n}$ behaves as

$$
\tau_{C}\left(k_{n}\right) \sim k_{n}^{-3 / 2}
$$

The exponent of this scaling law can be easily explained with a dimensional argument, by noticing that $<Y_{n}^{2}>\sim k_{n}$, and from (4.27) the characteristic time results to be just $\tau_{C}\left(k_{n}\right) \sim \sqrt{<Y_{n}^{2}>} /\left(k_{n}<Y_{n}^{2}>\right) \sim k_{n}^{-3 / 2}$.

Let us now discuss more interesting cases, i.e. the chaotic dissipative systems with non Gaussian statistics, called "shell models" which have been introduced to study the turbulent energy cascade and share many statistical properties with turbulent three dimensional velocity fields $[73,86]$. Let us consider a set of wave-numbers $k_{n}=2^{n} k_{0}$ with $n=0, \ldots, N$, and the shell-velocity (complex) variables $u_{n}(t)$ which must be understood as the velocity fluctuation over a distance $l_{n}=k_{n}^{-1}$. In the following we present numerical results for a particular choice, the so-called Sabra model $[87,86]$ :

$$
\left(\frac{d}{d t}+\nu k_{n}^{2}\right) u_{n}=i\left[k_{n} u_{n+1}^{*} u_{n+2}+b k_{n-1} u_{n+1} u_{n-1}^{*}+(1+b) k_{n-2} u_{n-2} u_{n-1}\right]+f_{n}
$$

where $b$ is a free parameter, $\nu$ is the molecular viscosity and $f_{n}$ is an external forcing acting only at large scales, necessary to maintain a stationary temporal evolution. We note that the shell models have the same type of quadratic nonlinearities as the Navier-Stokes equations in the Fourier space. In addition in the inviscid and unforced limit $\left(\nu=f_{n}=0\right)$ the energy $\left(\frac{1}{2} \sum_{n}\left|u_{n}\right|^{2}\right)$ is conserved and the volumes in phase space are preserved. Because of the above properties, the set of coupled ordinary differential equations (4.31) possesses the features necessary to mimic the Navier-Stokes non-linear evolution. The main, strong, difference with the previous inviscid models $(4.26,4.27)$ is the existence of a mean energy flux from large to small scales, which drives the system towards a strongly non-Gaussian stationary state [73]. The shell models 


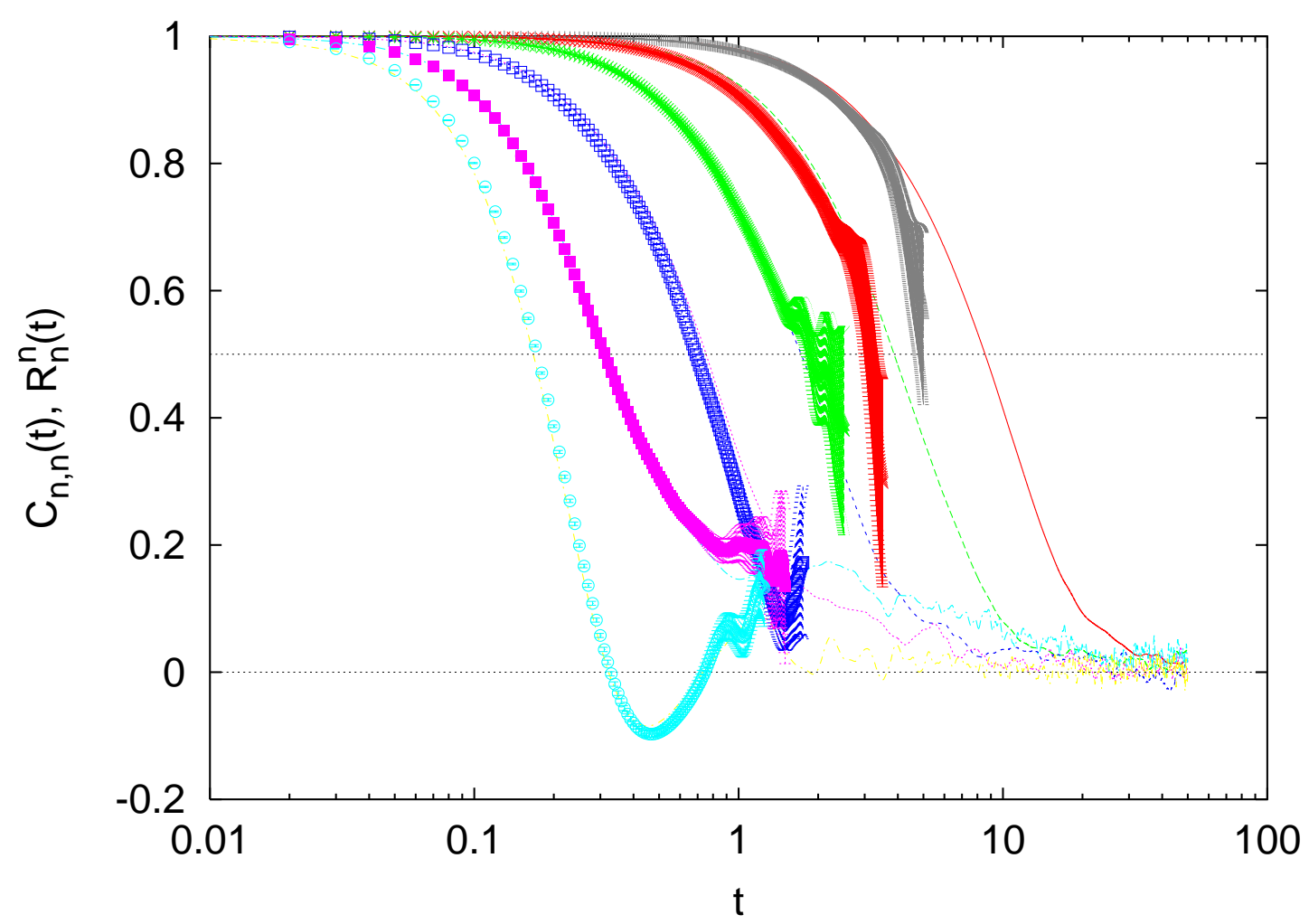

Fig. 1. FDRs for the six fastest variables of the model (4.27), with $N=20$, $\alpha=0.005$ and $\beta=1.7, n=5,6,7,8,9,10$. Thin lines represent the normalized correlation functions $C_{n, n}(t) / C_{n, n}(0)$. Self-response functions $R_{n, n}$ are plotted with statistically computed error bars.

here discussed present exactly the same qualitative difficulties as the original Navier-Stokes eqs.: strong non-linearity and far from equilibrium statistical fluctuations. The most striking quantitative feature of the fully developed turbulence, i.e. the highly non-Gaussian statistics and the existence of anomalous scaling laws for the velocity moments, is reproduced in shell models, in good quantitative agreement with the experimental results[73,86]. One has:

$$
<\left|u_{n}\right|^{p}>\sim k_{n}^{-\zeta(p)},
$$

where $\zeta(p) \neq \frac{p}{2} \zeta(2)$, which implies that the velocity PDF's at different scales cannot be rescaled by any change of variables.

Let us now examine the numerical results concerning the response functions in the shell model[85]. Figure 2 shows the normalized diagonal responses, $R_{n n}(t)$, for $k_{n}$ in the inertial range, $n \in[7,14]$. It is evident that the characteristic response time $\tau_{R}\left(k_{n}\right)$ decreases as $k_{n}$ increases.

In Figure 3, one sees a clear difference between the response and the correlation function: this is an additional indication that the inertial-range statistics are very far from Gaussian. Although the $R_{n n}(t)$ do not coincide with the (normalized) $C_{n n}(t) / C_{n n}(0)$, the times $\tau_{C}\left(k_{n}\right)$ and $\tau_{R}\left(k_{n}\right)$ are not very different $\tau_{C}\left(k_{n}\right) \propto \tau_{R}\left(k_{n}\right)$, in agreement with the numerical result obtained by 
Kraichnan with the DIA at moderate Reynolds numbers[80]. In addition, one has a scaling behavior $\tau_{R}\left(k_{n}\right) \propto \tau_{C}\left(k_{n}\right) \sim k_{n}^{-\gamma}$, where $\gamma$ is close to the value $2 / 3$ of the Kolmogorov scaling.

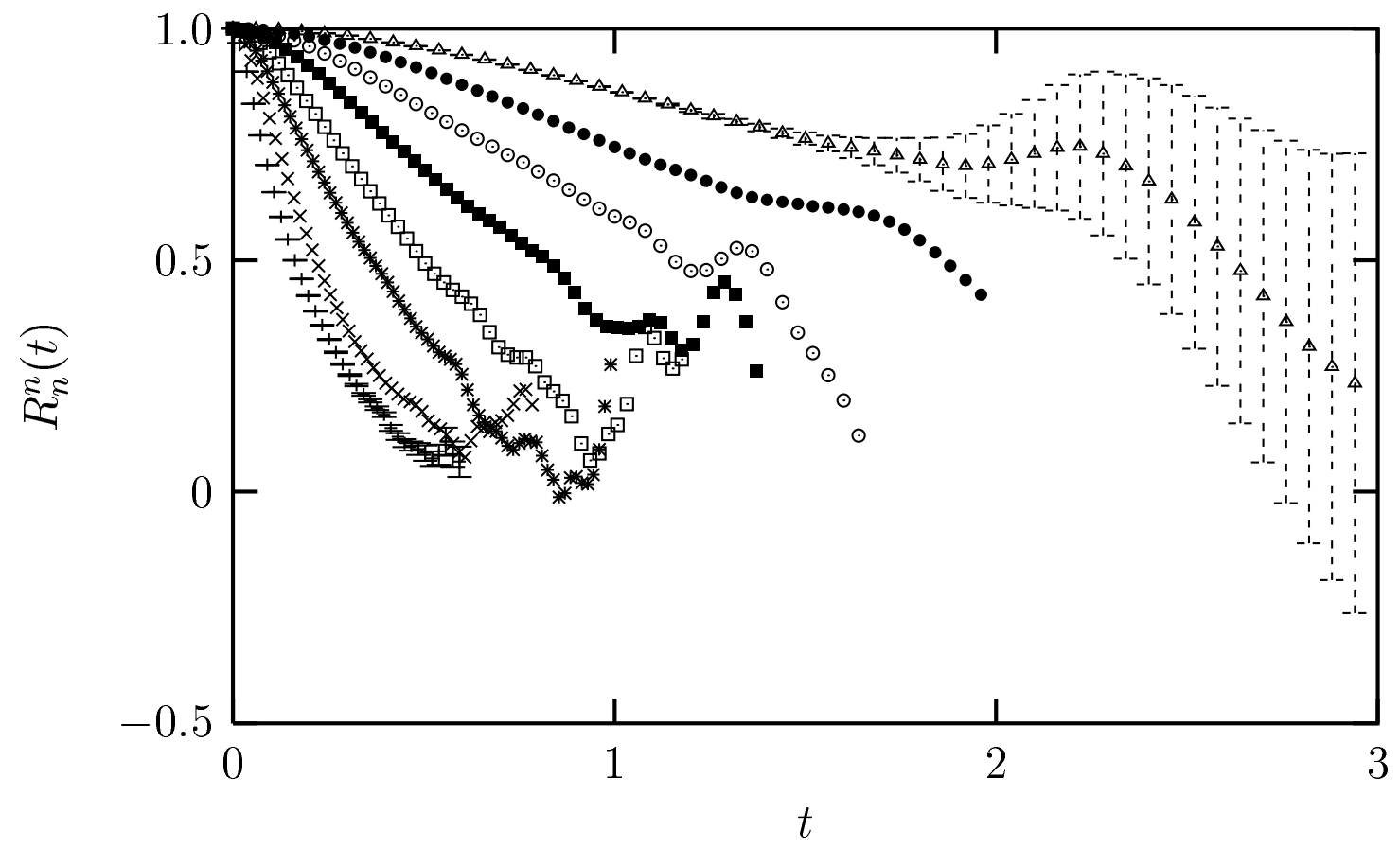

Fig. 2. Response functions, $R_{n, n}(t)$, for shells $n=7,8, \ldots, 13,14$ (from top to bottom). Error bars are shown only for the smallest and the largest scales. The parameters in eqs. (4.30) are $b=0.4, \nu=5.10^{-7}$, and $N=25$.

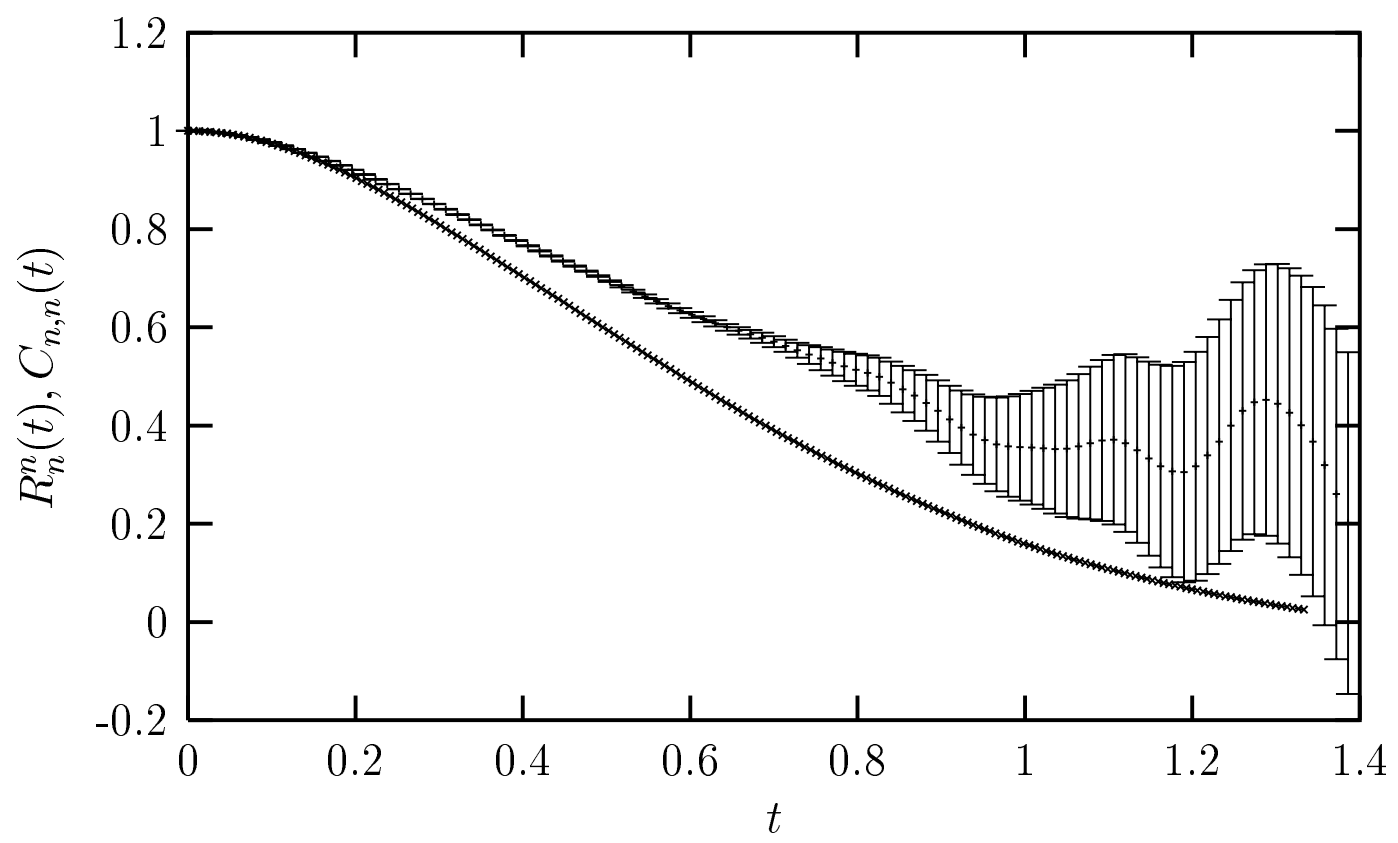

Fig. 3. Comparison between the averaged Response Function, $R_{n n}(t)$, (top) and the (normalized) self-correlation, $C_{n, n}(t) / C_{n n}(0)$ (bottom) for the shell $n=10$. 


\subsection{Beyond the linear FDR and climate}

In the case of finite perturbations, the FDR (3.6) is typically non-linear in the perturbation $\Delta \mathbf{x}_{0}$, and thus there are no simple relations analogous to (3.8). Nevertheless Eq. (3.6) guarantees the existence of a link between equilibrium properties of the system and the response to finite perturbations. This fact has a straightforward consequence for systems with a single characteristic time, e.g. a low dimensional system: a generic correlation function gives information about the relaxation time of finite size perturbations, even when the invariant measure $\rho$ is not known [35]. We saw that in systems with many different characteristic times, such as the fully developed turbulence, one has a more complicated scenario: different correlation functions show different behaviors. For a finite perturbation $\delta \mathbf{x}(0)$ such that $A(\mathbf{x}(0)+\delta \mathbf{x}(0))-A(\mathbf{x}(0))=\delta A(0)$, instead of (3.10) one has:

$$
\overline{\delta A(t)}=\langle A(\mathbf{x}(t)) F(\mathbf{x}(0), \delta \mathbf{x}(0))\rangle,
$$

therefore the relaxation properties depend explicitly on the initial perturbation $\delta \mathbf{x}(0)$ and not only on the value of $\delta A(0)$. In the following we will discuss the relevance of the amplitude of the perturbation, which may play a major role in determining the response, because different amplitudes may trigger different response mechanisms with different time scales $[35,85]$.

\subsubsection{Response to finite perturbations}

Consider two cases with a single characteristic time: a low dimensional deterministic chaotic system, known as the Lorenz model, and a nonlinear Langevin equation. We begin with the Lorenz model [88]:

$$
\begin{aligned}
& \frac{d x}{d t}=\sigma(y-x) \\
& \frac{d y}{d t}=-x z+r x-y \\
& \frac{d z}{d t}=x y-b z
\end{aligned}
$$

with standard parameters for chaotic behavior: $b=8 / 3, \sigma=10$ and $r=28$. The correlation function of the variable $z$, shown in Figure 4 , qualitatively reproduces the behavior of the response to different sizes of the perturbation of the $z$ variable, ranging from infinitesimal ones up to those of the size of the attractor. The agreement between the response function and the normalized correlation function is just qualitative and it does not depend too much on the amplitude of the perturbation, because the invariant distribution is not 
Gaussian (see inset of Fig. 4) and the general correlation (3.13) should be used.

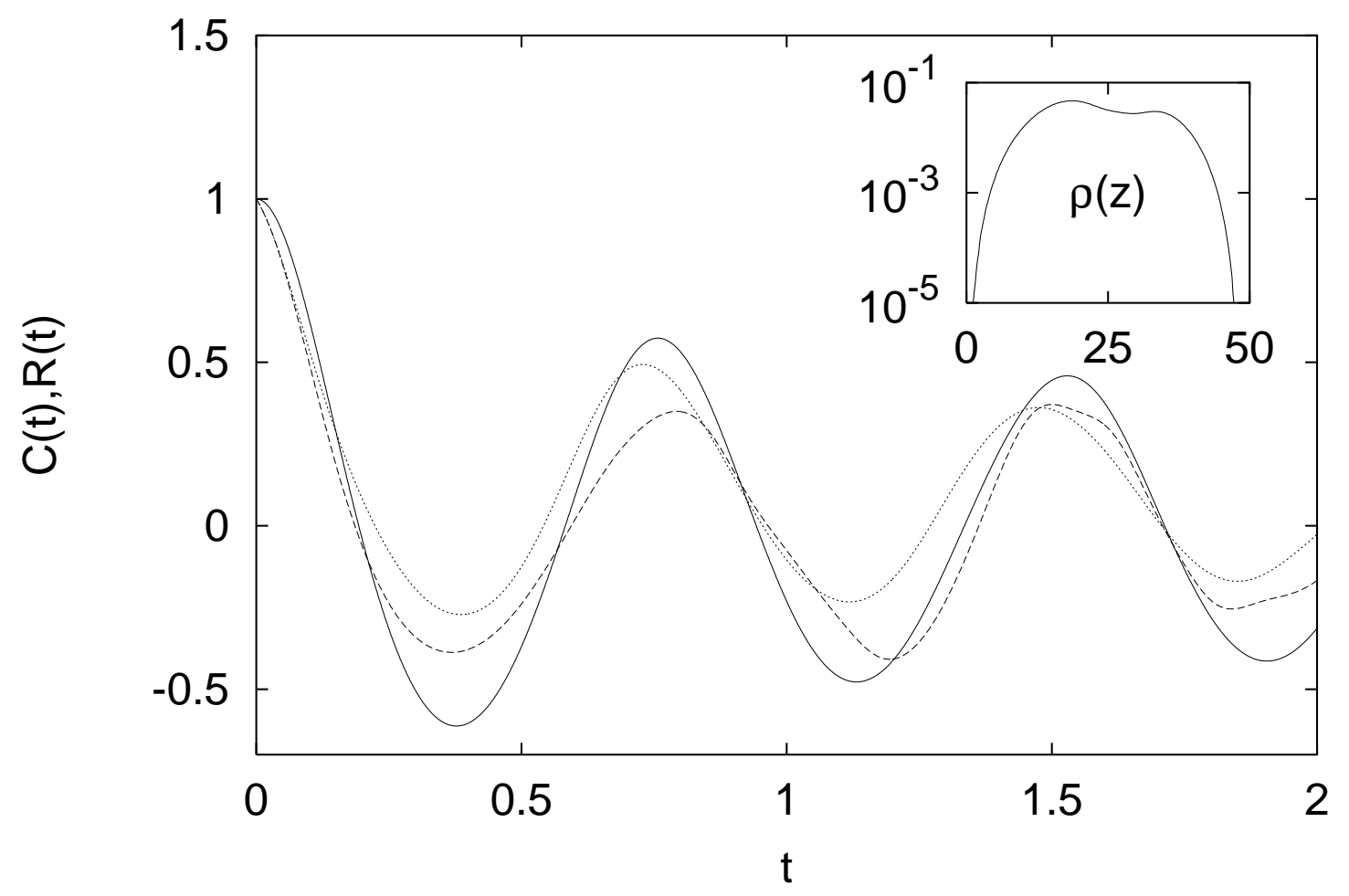

Fig. 4. Normalized correlation function of the $z$ variable of Lorenz model (solid line) compared with the mean response to different perturbations of the same variable. $\delta z_{0}=10^{-2} \sigma$ (dashed line), $\delta z_{0}=\sigma$ (dotted line), with $\sigma^{2}=\left\langle z^{2}\right\rangle-\langle z\rangle^{2}=75.17$. In the inset the invariant probability $\rho(z)$ versus $z$. Being a 1D projection, $\rho(z)$ results regular, even if the full $3 \mathrm{D}$ distribution is fractal.

To better illustrate this point, we consider a system whose invariant probability distribution is known. In this case, we can quantitatively compare the differences between the responses to infinitesimal and finite perturbations. Let us consider the stochastic process $x(t)$ described by

$$
\frac{d x}{d t}=-\frac{d U(x)}{d x}+\sqrt{2 D} \eta(t)
$$

where $\eta(t)$ is a white noise. The invariant probability distribution is [13]:

$$
\rho(x)=\mathcal{N} e^{-U(x) / D}
$$

where $\mathcal{N}$ is fixed by normalization, and

$$
U= \begin{cases}\frac{1}{2} x^{2} & ,|x|<1 \\ |x|-\frac{1}{2}, & |x|>1\end{cases}
$$


The resulting pdf, shown in the inset of Fig. 5, has a Gaussian core, with exponential tails. Figure 5 also shows the response function for an infinitesimal and for a finite size perturbation. For both perturbations, the response function measured from the perturbed trajectories is exactly predicted by the statistics of the unperturbed system according to (3.13), while the Gaussian correlation $C(t)=\langle x(t) x(0)\rangle / \sigma^{2}$ is only an approximation of the response. By construction, the pdf of this system has larger tails than in the Gaussian case, thus large fluctuations have slower decay than small ones. On the contrary in the Gaussian case, i.e. $U(x)=\frac{1}{2} x^{2}$ for any $x$, the response is an exponential function and does not depend on the amplitude of the initial perturbation $\delta x(0)$.

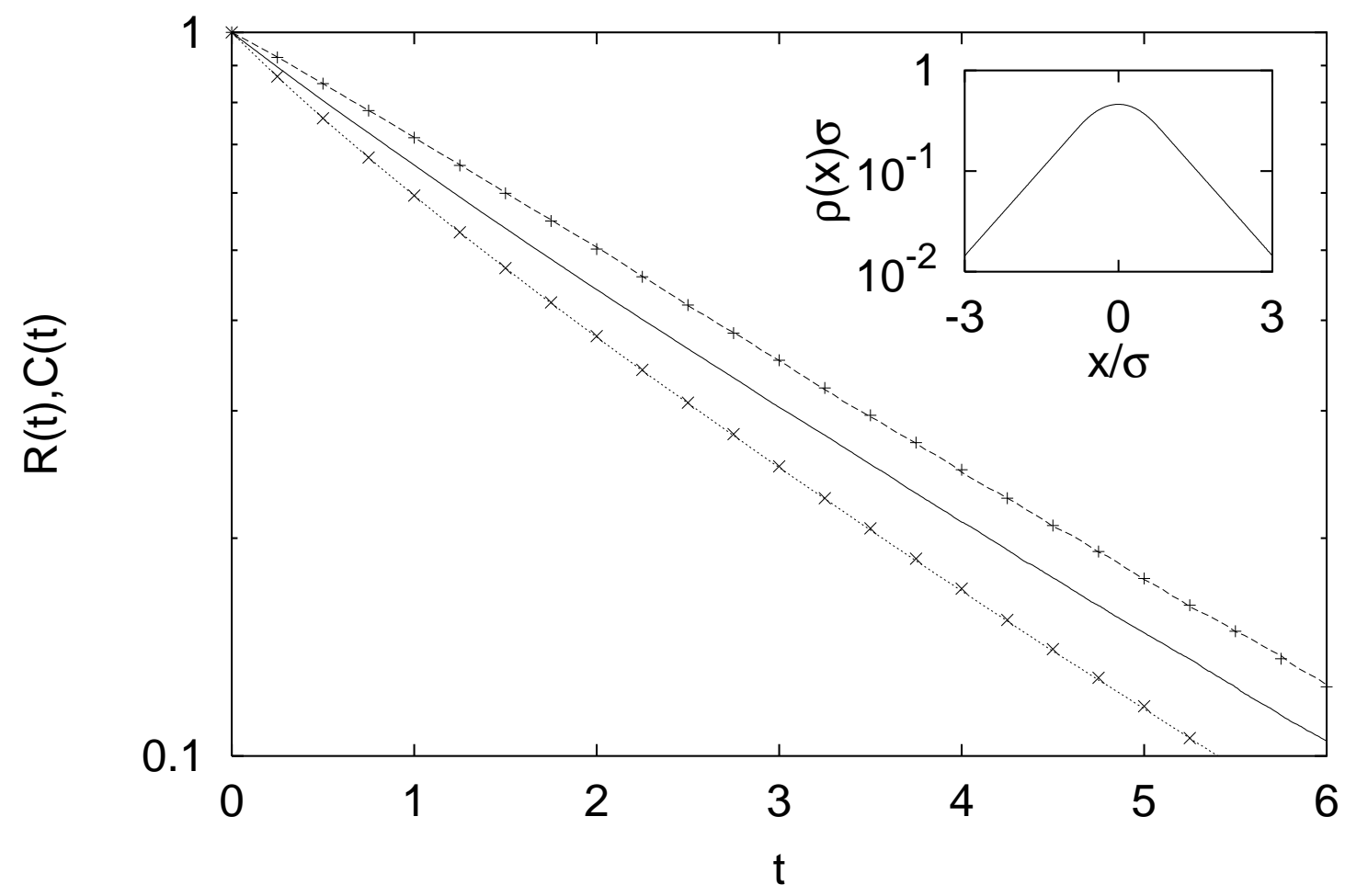

Fig. 5. Response of the stochastic differential equation (4.34) with $D=1$ and $U(x)$ as in (4.36), to different perturbations: large $\delta x_{0}=2.3 \sigma(+)$ and infinitesimal $\delta x_{0}=7.6 \times 10^{-3} \sigma(\times)$. In both cases the mean response is exactly predicted by the correlation function $\langle x(t) f(x(0))\rangle$, with $f(x)$ given by $(3.6,3.7)$, dashed line for $\delta x_{0}=2.3 \sigma$ and dotted line for $\delta x_{0}=7.6 \times 10^{-3}$. The correlation function $\langle x(t) x(0)\rangle / \sigma^{2}$ (solid line) is shown for comparison. In the inset the invariant probability distribution $\rho(x) \sigma$ versus $x / \sigma$ with $\sigma^{2}=\left\langle x^{2}\right\rangle-\langle x\rangle^{2}=1.15$.

We have already seen how in systems with many characteristic times, different correlation functions behave differently and a variety of time scales emerges, which correspond to the different decay times of the correlation functions. In addition, at variance with systems with a single time scale, the amplitude of the perturbation plays a major role in determining the response, because 
different amplitudes affect features characterized by different time scales. To illustrate these ideas, we refer to the shell model (4.30) and consider the total energy $E(t)=\frac{1}{2} \sum_{n=1}^{N}\left|u_{n}(t)\right|^{2}$ which is conserved in the inviscid, unforced limit [73]. We study the response to perturbations of $E$ with different amplitude, of the following form:

$$
\left|\delta u_{n}^{(i)}(0)\right|=\left\{\begin{array}{ll}
0 & , 1 \leq n \leq i-1 \\
\sqrt{\left\langle\left|u_{n}\right|^{2}\right\rangle} & , i \leq n \leq N
\end{array} .\right.
$$

The value of $i=1, . ., N$ determines the largest spatial scale $\ell$ of the perturbation, $\ell \sim k_{i}^{-1}$. This corresponds to a set of initial perturbations of the energy

$$
\delta E^{(i)}(0)=\frac{1}{2} \sum_{n=i}^{N}\left\langle\left|u_{n}\right|^{2}\right\rangle .
$$

Such a kind of perturbation is motivated by the fact that, in the unperturbed system, the energy is distributed among the shells according to the Kolmogorov scaling $\left\langle\left|u_{n}\right|^{2}\right\rangle \sim k_{n}^{-2 / 3}$, and the smaller scales give smaller contributions to the energy $E(t)$. Thus it is natural to assume that a small perturbation of the energy affects mainly the small scales.

For each perturbation $\delta E^{(i)}(0)$, the average energy response

$$
R_{E}(t)=\frac{\overline{\delta E^{(i)}(t)}}{\delta E^{(i)}(0)}
$$

reveals a behavior similar to the correlation function of the largest perturbed shell $u_{i}(t)$, as shown in Fig. 6. An additional measure of the relaxation time is provided by the halving times $T_{h}$ of the mean response, when $\overline{\delta E^{(i)}\left(T_{h}\right)}=$ $\frac{1}{2} \delta E^{(i)}(0)$. The dependence of the halving times on the amplitude of the initial perturbation reflects the Kolmogorov scaling for characteristic times. Noting that $\tau_{C}\left(k_{n}\right) \sim \tau_{R}\left(k_{n}\right) \sim k_{n}^{-2 / 3} \sim u_{n}^{2} \sim \delta E^{(i)}(0)$ one has:

$$
T_{h} \sim \delta E^{(i)}(0)
$$

The above results show that the response of a system with many characteristic times to a finite size perturbation may depend on the amplitude of the perturbation. Thanks to the applicability of the FDR, it is possible to establish a link between relaxation times of different perturbations and characteristic times of the system.

\subsubsection{FDR and climate}

One of the key issues in climate modeling is the study of the response to perturbations of the external forces, or control parameters. In such a context, the 


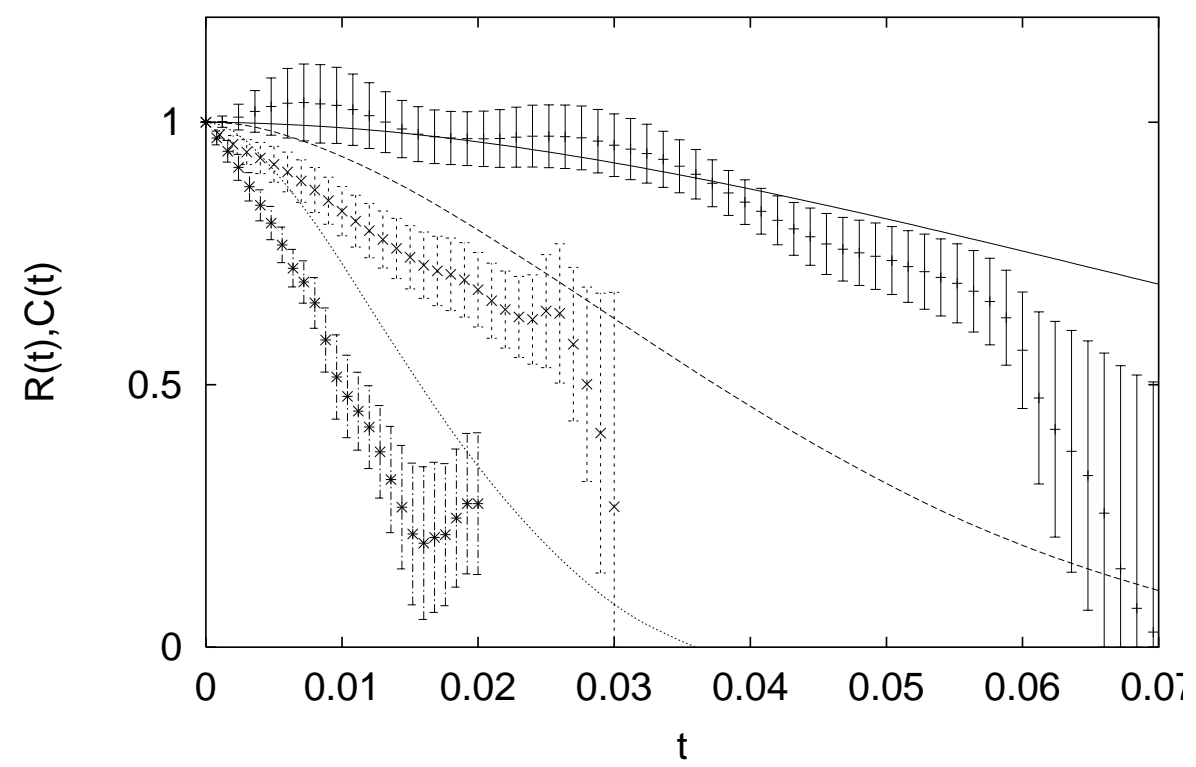

Fig. 6. Mean response $R_{E}(t)$ of the total energy $E(t)=\frac{1}{2} \sum\left|u_{n}\right|^{2}$ of the shell model (4.30) to different amplitude perturbations: $\delta E^{(12)}(0)=5.5 \times 10^{-3}(+)$, $\delta E^{(14)}(0)=1.7 \times 10^{-3}(\times), \delta E^{(16)}(0)=4.5 \times 10^{-4}(*)$; and the (normalized) correlation functions of the corresponding largest perturbed shell: shell $n=12$ (solid line), shell $n=14$ (dashed line), shell $n=16$ (dotted line).

importance of the FDR is rather obvious: if it holds there is the possibility, at least in principle, to understand the behavior of the system under perturbations (e.g. a volcanic eruption, or change of the $\mathrm{CO}_{2}$ concentration) in terms of the knowledge obtained from its past time history[70,71].

To describe the climate system, let us consider the symbolic evolution equation:

$$
\frac{d \mathbf{X}}{d t}=\mathbf{Q}(\mathbf{X})+\mathbf{f}(t)
$$

where $\mathbf{X}$ is the state vector for the system, $\mathbf{Q}(\mathbf{X})$ represents complicated dynamical processes and $\mathbf{f}(t)$ the external influences. The average effect of an infinitesimal perturbation $\delta \mathbf{f}(t)$ can be written in terms of the response matrix $R_{\alpha, \beta}(t)$; if $\delta \mathbf{f}(t)=0$ for $t<0$ one has:

$$
\overline{\delta X_{\alpha}(t)}=\sum_{\beta} \int_{0}^{t} R_{\alpha, \beta}\left(t-t^{\prime}\right) \delta f_{\beta}\left(t^{\prime}\right) d t^{\prime} .
$$

The goal is to write $R_{\alpha, \beta}(t)$ in terms of correlation functions of the unperturbed system.

Under many points of view, the questions concerning the FDR in climate systems are rather similar to those posed in fluids dynamics, because one deals with non Hamiltonian and non linear systems, whose invariant measures are 
non-Gaussian. On the other hand, in climate studies, one hardly faces equations obtained from first principles, and typically it is necessary to work with rather crude models, or with experimental time series [89]. Moreover, in climate problems and in geophysics, the interest in infinitesimal perturbations is rather academic, while the interesting problem is the behavior of the relaxation of large fluctuations, due to sudden changes of the parameters.

Starting from the seminal works of Leith [70,71], who proposed to employ the FDR to study the response of climatic systems to changes in the external forcing, many authors applied this relation to different geophysical problems, ranging from simplified models [72], to general circulation models [90,91] and to the covariance of satellite radiance spectra [92]. In most of these attempts, the FDR has been used in its Gaussian version, eq. (3.14), which has been acritically considered a reasonable approximation, without investigating its limits of applicability. For a recent application to climate models with fast and slow variables see [93].

The results previously discussed show that a FDR holds also for non infinitesimal perturbations. This generalization of the usual linear response theory has an obvious interest for climate applications. Although the FDR in its simplest form (3.14) does not hold, one has, at least, a qualitative agreement between responses and correlations. In particular, from features of the unperturbed dynamics, one infers the presence of different response times associated with different variables. This description is qualitatively satisfactory. But a precise quantitative estimate faces difficult technical problems such as:

a) the FDR involves the detailed form of the invariant probability distribution (which is usually unknown)

b) non infinitesimal perturbations require good statistics to resolve the rare events.

\subsection{Granular materials}

Granular systems $[94,95,96]$ constitute an example of non-equilibrium physics, pervading our everyday life: sand, sugar, coffee, pills, seeds, powders, etc., are all instances of the "granular state of matter". For the behavior of granular systems, the environment temperature plays only a marginal role. At the same time these systems are made of relatively many particles. This suggests that statistical mechanical methods, such as kinetic theory, hydrodynamics, thermodynamics and linear response can be adapted to granular materials.

A starting point of the statistical approach is to identify a distribution that is left invariant by the dynamics, as is the case of the microcanonical distribution in Hamiltonian systems, and the conditions under which this distribution 
can be reached, starting from a generic initial condition. Unfortunately, statistically steady states of granular matter are usually given by the balance of non-conservative forces, such as external tapping and inelastic collisions or friction with the boundaries, and the corresponding invariant measures are not known. It is therefore natural to ask whether it is possible to speak at all of a granular temperature. This question has different answers, depending on the degree of diluteness of the granular system under examination. The most straightforward definition comes from the case of dilute, strongly vibrated granular systems, which reach a non-equilibrium stationary state: by analogy with molecular gases, a "granular temperature" $T_{g}$ can be defined in terms of the average local kinetic energy per particle. This approach can a priori be extended to denser, liquid-like, strongly vibrated systems [97]. However, if the density is further increased, or the external energy injection strongly reduced, the assumption of ergodicity becomes less and less obvious: reaching a stationary state may become experimentally or numerically very difficult. Dense granular systems exhibit aging [98,99] and memory [100,101]. Analogies with other aging systems have also led to the definition of dynamic temperatures as quantifying the violation of the Hamiltonian version of the fluctuation-dissipation theorem [102]. In this review, we do not touch on nonergodic statistical physics, hence we do not discuss glassy granular systems and we focus only on systems that rapidly forget their initial conditions. This class includes the so-called "granular gases", i.e. granular systems which are fluidized by different means, for instance strongly shaking the container, or otherwise letting a low-density fluid (e.g. air) pass around the grains at high velocity.

In Appendix A the reader can find a brief review of the models used for the granular gases. Such models include the following ingredients: a gas of particles, a collision rule that dissipates kinetic energy, and a dynamical rule governing the dynamics between collisions. This dynamics depends upon the external forcing. If the system is driven from the boundaries (shaking the container) and gravity can be neglected, for example in microgravity experiments, the bulk dynamics is made of straight paths plus inelastic collisions. If the container is large, the external energy may take a long time to reach the interior of the gas: this motivates the study of pure cooling models [103]. They are considered as the building blocks of the hydrodynamics of boundary driven granular gases [104]. For this reason, several authors have considered the application of the theory of linear response to perturbations of these systems, as a valid alternative to kinetic theory, for the derivation of transport coefficients [105]. Here, the analysis is more difficult because there is no steady state, but only a scaling behavior towards a state with all the particles at rest, which undermines basic assumptions of kinetic theory, such as the separation of scales and the fast equilibration of microscopic modes [106,107]. On the other hand, experiments with uniform drivings, i.e. setups where the injection of energy reaches all grains, show a very rich phenomenology and are much 
easier to model: the gas reaches a statistically steady state and the formalism introduced in Sec. 3 can be applied.

\subsubsection{Cooling granular gases}

Most of the work devoted to linear response in cooling granular gases, concerns the calculation of transport coefficients for small perturbations of the homogeneous cooling state, under different assumptions [108,109,110,105]. A well established approach $[111,112]$ considers the Boltzmann equation for a granular gas as the starting point, see Appendix A, Eq. (A.3) with $\Gamma=\gamma=0$, which describes the evolution of a single particle velocity distribution $f(\mathbf{x}, \mathbf{v}, t)$, in a gas with particles interaction given by inelastic collisions, obeying the rule of Eq. (A.1). The effect of collisions is included in the collision integral, Eq. (A.4).

Following the standard kinetic theory procedure, it is possible to derive balance equations for the usual fields:

$$
\begin{aligned}
D_{t} n+n \nabla \cdot \mathbf{u} & =0 \\
D_{t} u_{i}+(m n)^{-1} \nabla_{j} P_{i j} & =0 \\
D_{t} T_{g}+\frac{2}{d n}\left(P_{i j} \nabla_{j} u_{i}+\nabla \cdot \mathbf{q}\right)+T_{g} \zeta & =0,
\end{aligned}
$$

where $n, \mathbf{u}$ and $T_{g}$ are the density, velocity and temperature fields respectively, and $D_{t}=\partial_{t}+\mathbf{u} \cdot \nabla$. The pressure tensor $P_{i j}$, the heat flux $\mathbf{q}$ and the cooling coefficient $\zeta$ depend upon the particular (time dependent) form of the distribution function $f(\mathbf{x}, \mathbf{v}, t)$. A homogeneous scaling solution $f_{0}(\mathbf{x}, \mathbf{v}, t)=$ $n v_{0}^{-d}(t) f_{0}^{*}\left(\mathbf{v} / v_{0}(t)\right)$ is supposed to exist: it has no $\mathbf{x}$-dependence (homogeneity) and the time-dependence is all included in the thermal velocity $v_{0}(t)=$

$\sqrt{2 T_{g}(t) / m}$ [113]. Such a solution must satisfy an equation for $T_{g}(t)$, which results in the so-called Haff law [114] for the homogeneous granular cooling. At large times, in the homogeneous case, the granular temperature decays as $T_{g}(t) \sim t^{-2}$.

It is possible to express a generic perturbation of the scaling state $f_{0}$ in terms of the gradients of the macroscopic fields $n(\mathbf{x}, t), \mathbf{u}(\mathbf{x}, t), T_{g}(\mathbf{x}, t)$. Substituting this form in the Boltzmann equation and retaining only terms of first order in these gradients results in expressions for the pressure tensor and the heat flux, which are linear in the field gradients [111]:

$$
\begin{aligned}
P_{i j} & =p(\mathbf{x}, t) \delta_{i j}-\eta\left(\nabla_{j} u_{i}+\nabla_{i} u_{j}-\frac{2}{d} \delta_{i j} \nabla \cdot \mathbf{u}\right) \\
\mathbf{q} & =-\kappa \nabla T_{g}-\mu \nabla n
\end{aligned}
$$

where $\mu$ is a new coefficient peculiar to granular gases, which vanishes in 
the elastic limit, and the following "Green-Kubo" relations hold, giving the transport coefficients:

$$
\begin{aligned}
& \eta(t)=\frac{2 n m \ell v_{0}(t)}{d^{2}+d-2} \int_{0}^{s(t)} d s^{\prime} \sum_{i j}\left\langle D_{i j}^{*}\left(s^{\prime}\right) \Phi_{2, i j}^{*}\right\rangle \mathrm{e}^{-\frac{s^{\prime} \zeta^{*}}{2}} \\
& \kappa(t)=\frac{n \ell v_{0}(t)}{d} \int_{0}^{s(t)} d s^{\prime}\left\langle\mathbf{S}^{*}\left(s^{\prime}\right) \cdot \boldsymbol{\Phi}_{3}^{*}\right\rangle \mathrm{e}^{\frac{s^{\prime} \zeta^{*}}{2}} \\
& \mu(t)=\frac{2 T_{g}(t) \kappa(t)}{n}+\frac{m \ell v_{0}^{3}(t)}{d} \int_{0}^{s(t)} d s^{\prime}\left\langle\mathbf{S}^{*}\left(s^{\prime}\right) \cdot\left(\boldsymbol{\Phi}_{1}^{*}-\boldsymbol{\Phi}_{3}^{*}\right)\right\rangle .
\end{aligned}
$$

The transport coefficients depend upon the time $t$, which is related to the time $s$ through the transformation $d s=d t v_{0}(t) / \ell$, with $\ell$ the mean free path, i.e. $s$ is proportional to the total number of collisions occurred since the initial time. In the above expressions the transport coefficients are obtained as time integrals of correlation functions calculated with the unperturbed distribution $f_{0}$ (the average $\langle\ldots\rangle$ refers to this distribution). These are correlations between the currents at time $s^{\prime}$

$$
\begin{aligned}
D_{i j}^{*} & =V_{i}^{*} V_{j}^{*}-\frac{1}{d} V^{* 2} \delta_{i j} \\
\mathbf{S}^{*} & =\left(V^{* 2}-\frac{d+2}{2}\right) \mathbf{V}^{*}
\end{aligned}
$$

and new conjugated quantities at time 0:

$$
\begin{aligned}
\boldsymbol{\Phi}_{1}^{*} & =\frac{1}{2}\left(\mathbf{V}^{*}+\frac{1}{2} \frac{\partial}{\partial \mathbf{V}^{*}} \ln f_{0}^{*}\right) \\
\Phi_{2, i j}^{*} & =\frac{1}{2}\left(\frac{1}{d} \delta_{i j} \mathbf{V}^{*} \cdot \frac{\partial}{\partial \mathbf{V}^{*}}-V_{i}^{*} \frac{\partial}{\partial V_{j}^{*}}\right) \ln f_{0}^{*} \\
\boldsymbol{\Phi}_{3}^{*} & =\frac{1}{2}\left[\frac{\partial}{\partial \mathbf{V}^{*}} \ln f_{0}^{*}-\mathbf{V}^{*}\left(d+\mathbf{V}^{*} \cdot \frac{\partial}{\partial \mathbf{V}^{*}} \ln f_{0}^{*}\right)\right] .
\end{aligned}
$$

The rescaled peculiar velocity $\mathbf{V}^{*}=(\mathbf{v}-\mathbf{u}(\mathbf{x}, t)) / v_{0}(t)$ and the rescaled cooling coefficient $\zeta^{*}=\zeta \ell / v_{0}$ have been used in the above expressions. In the elastic limit $r \rightarrow 1$, the unperturbed state $f_{0}$ becomes the Maxwell distribution, so that $\boldsymbol{\Phi}_{1}^{*} \rightarrow 0, \Phi_{2, i j}^{*} \rightarrow D_{i j}^{*}, \boldsymbol{\Phi}_{3}^{*} \rightarrow \mathbf{S}^{*}(\mathbf{V})$, and therefore

$$
\begin{aligned}
\mu \rightarrow \mu_{0} & =0 \\
\eta \rightarrow \eta_{0} & =\frac{2 n m \ell v_{0}(t)}{d^{2}+d-2} \int_{0}^{s} d s^{\prime} \sum_{i j}\left\langle D_{i j}^{*}\left(s^{\prime}\right) D_{i j}^{*}(0)\right\rangle \\
\kappa \rightarrow \kappa_{0} & =\frac{n \ell v_{0}(t)}{d} \int_{0}^{s} d s^{\prime}\left\langle\mathbf{S}^{*}\left(s^{\prime}\right) \cdot \mathbf{S}^{*}\right\rangle .
\end{aligned}
$$


The difference occurring in the granular case $(r<1)$, with respect to the elastic case, are the following: the averages $\langle\ldots\rangle$ are based on an invariant measure $f_{0}$ which is not Maxwellian; the conjugated currents at time 0 are different from the fluxes $\mathbf{S}^{*}$ and $D_{i j}^{*}$, as expected from the non-Gaussianity of the unperturbed state and from the considerations of section 3. The time integration is replaced by an integration over the average collision number $s$, and contains an additional time dependent factor. This term arises from the change of the temperature over the duration of the integral.

\subsubsection{Driven granular gases}

The effect of a uniform driving acting upon all the particles of the granular gas is modeled assimilating the driving to a thermal bath, on the macroscopic scale of grains (see Appendix A for details). All the variants of this thermal bath model guarantee the achievement of a statistically stationary state after the time needed to forget the state in which the system has been initially prepared. The study of the FDR in these models is recent and has been carried out by means of numerical simulations $[115,116,117,118]$. Some of the numerical results has received an analytical interpretation in [119], and experimental verifications have been attempted in [120]. Other idealized models of inelastic energy exchange $[121,122,123]$ have also been proposed. Some of the numerical results and their interpretation are reviewed here, in view of relation (3.9). We start with monodisperse weakly correlated systems, we continue with the bidisperse case, and we conclude with an analysis of the effect of strong correlations in dense systems.

\section{Monodisperse gases with weak correlations}

A simple Fluctuation-Response experiment involving only one particle of a statistically stationary gas made of identical particles, consists in applying an instantaneous perturbation $\delta v_{0}(0)$ to the $x$-component of the velocity of that particle at a time 0 (assumed to be in the stationary regime), and observing its relaxation averaged over many different realizations:

$$
R(t)=\frac{\overline{\delta v_{x}(t)}}{\delta v_{x}(0)}
$$

If the invariant measure describing the statistically stationary state satisfies the following condition (the independence of the degrees of freedom)

$$
\rho\left(\left\{\mathbf{v}_{i}, x_{i}\right\}\right)=n^{N} \prod_{i=1}^{N} \prod_{\alpha=1}^{d} p_{v}\left(v_{i}^{\alpha}\right)
$$

where $v_{i}^{\alpha}$ is the $\alpha$ velocity component of the $i$-th particle, with $n=N / V$, and 
if the single particle velocity pdf $p_{v}(v)$ is a Gaussian, then Eq. (3.9) predicts

$$
R(t)=C_{1}(t)=\frac{\left\langle v_{x}(t) v_{x}(0)\right\rangle}{\left\langle v_{x}^{2}\right\rangle}
$$

for the response function. If one of these assumptions does not apply, then relation (4.61) is no more expected to hold.

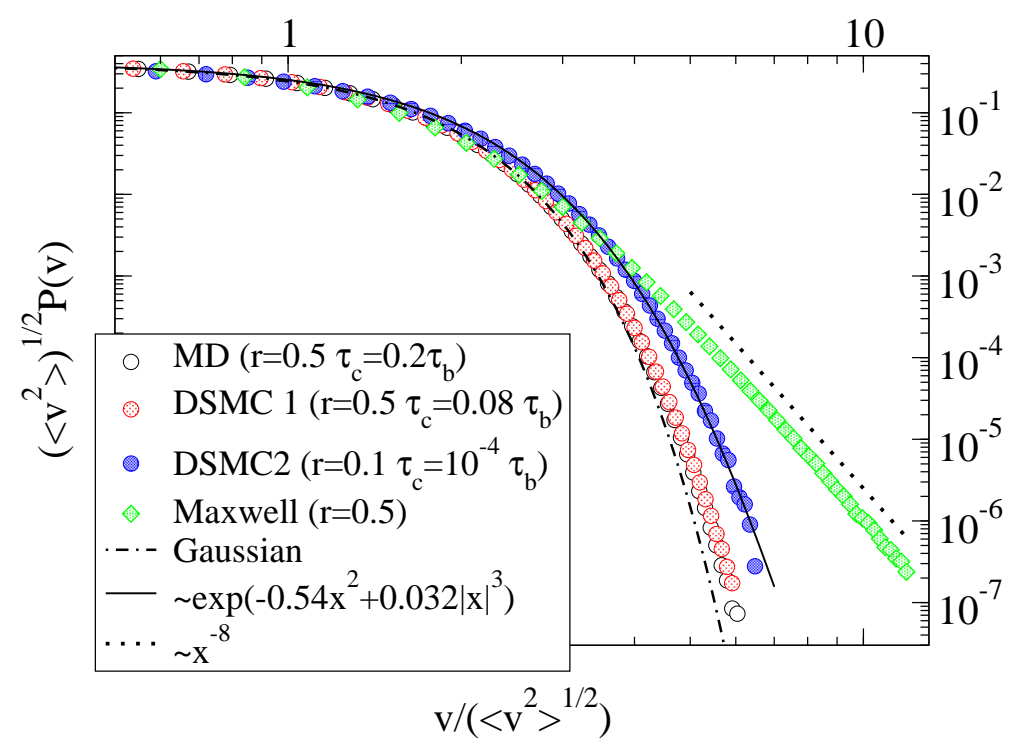

Fig. 7. Dilute cases, velocity pdf in different cases.

The above recipe has been applied $[115,116,118]$ to three monodisperse variants of the general model of driven granular gases of Appendix A, Eq. (A.2), characterized by:

- i) very dilute conditions in $2 D$, with volume fraction $\phi=n \frac{\sigma^{2}}{4}<0.1$ (being $\sigma$ the diameter if the hard disks) and mean free time between collisions $\tau_{c}$ not much smaller than the viscosity time $\tau_{b}=1 / \gamma$;

- ii) same as model i) but with the additional assumption that positions of particles are irrelevant and the probability of a collision is proportional to the relative velocity (Direct Simulation Monte Carlo, DSMC);

- iii) same as model ii), with a constant collision probability (inelastic Maxwell model) and a deterministic driving.

The last two variants are discussed in details in Appendix A. All these models have the common property (4.60) and non-Gaussian velocity pdfs $p_{v}(v)$. In particular model iii) displays $p_{v}(v)$ with high energy tails of the form $v^{-b}$ with $b=4$ in $d=1[124,125]$ and $b>4$ in $d=2$ (a good estimate for not too high inelasticity is $b \simeq 4 /(1-r)[126])$. The extreme simplification of the dynamics of model iii) allows us to obtain a direct analytical computation of time correlations and responses (see Appendix A). In Figure 7, some examples of the velocity pdfs for these different models are shown. In Molecular Dynamics 
(MD) simulations of inelastic hard disks, even if strongly inelastic, but still dilute $(\phi<0.1)$, the velocity pdf is not far from a Gaussian, as in a DSMC with similar choices of the parameters $\left(\tau_{c} \sim 0.1 \tau_{b}\right)$. Increasing $n$ in the DSMC leads to stationary regimes very far from thermal equilibrium, with $T_{g} \ll T_{b}$ and larger tails of the velocity pdf $p_{v}(v)$. A convenient fit of these velocity pdfs is the following

$$
p_{v}(v)=c_{0} \exp \left(-c_{1} v^{2}+c_{2}\left|v^{3}\right|-c_{3} v^{4}\right)
$$

where $c_{0}$ is the normalization constant. In most of observed cases $\left|c_{3} v_{\text {max }}^{4}\right| \ll$ $\left|c_{2} v_{\text {max }}^{3}\right|$, with $v_{\max }$ the largest value of $v$ in the histogram. Therefore, in practice, we have a rather good fit even dropping the quartic term. Using relation (3.9) with the assumption of uncorrelated velocities, a prediction for the response is obtained, in the form

$$
\begin{array}{r}
R(t)=-2 c_{1}\left\langle v_{x}(t) v_{x}(0)\right\rangle+3 c_{2}\left\langle v_{x}(t)\left|v_{x}(0)\right| v_{x}(0)\right\rangle \\
=-2 c_{1}\left\langle v_{x}^{2}\right\rangle C_{1}(t)+3 c_{2}\left\langle\left|v_{x}(0)^{3}\right|\right\rangle C_{2}(t)
\end{array}
$$

which also defines $C_{2}(t)$.

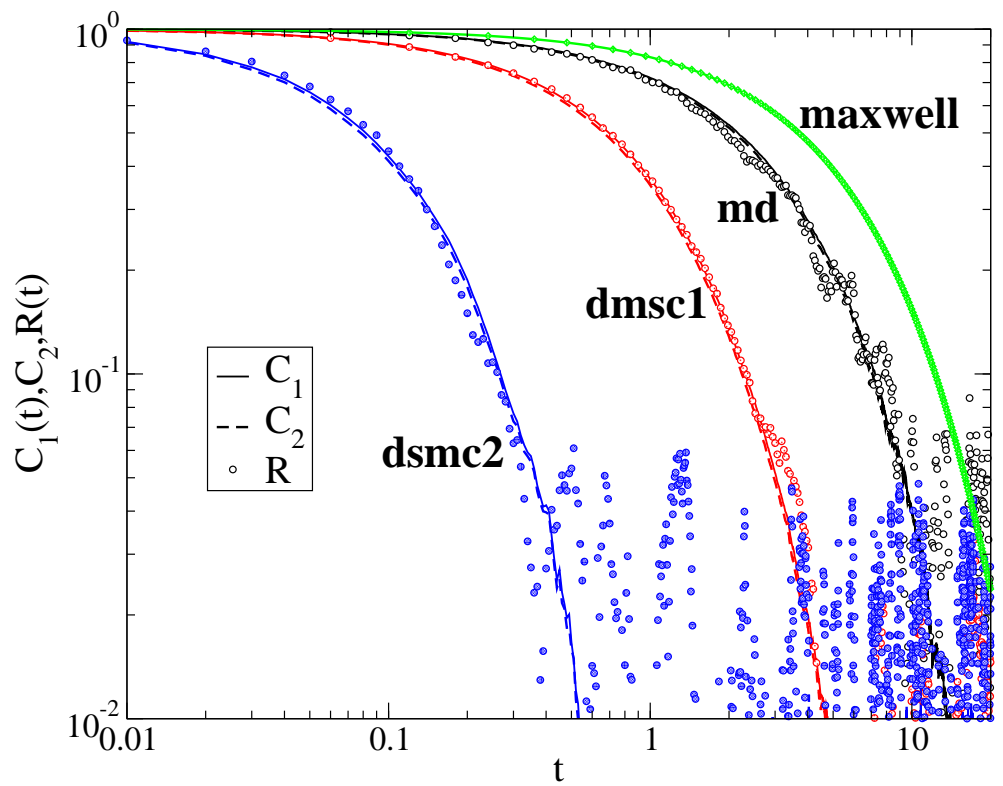

Fig. 8. Dilute cases; experiment with instantaneous displacement of the velocity of a tracer.

Typical results of Fluctuation-Response numerical experiments on these three models are shown in Figure 8, together with the normalized velocity correlations $C_{1}(t)$ and $C_{2}(t)$. The general picture that emerges from these numerical experiments is the following:

- different correlations practically display the same behavior (we do not show $C_{3}(t)=\left\langle v(t) v^{3}(0)\right\rangle /\left\langle v(0)^{4}\right\rangle$, but the result is similar) 
- a very good agreement between $R(t)$ and $C_{1}(t)$ is observed, which amounts to a verification, within the limits of numerical precision, of the Einstein relation.

The observation that the self-correlations $C_{k}(t)$ are almost identical, at least for $k=1,2,3$, is very robust: indeed with large statistics one can appreciate very small differences only at late times. Anyway, the data on the response function is usually quite noisy, and, for the practical purpose of the linear combination involved in the response, these small differences are negligible: the Einstein relation is always satisfied, with $T_{g}$ as a proportionality factor. In conclusion, the coincidence between velocity correlations of different orders makes the non-Gaussianity of the velocity pdf irrelevant for the linear response of the system.

It is interesting to note that an almost identical situation is encountered in a gas of non-interacting particles, whose velocities obey a Langevin equation with a non-quadratic potential:

$$
\frac{d v(t)}{d t}=-\gamma \frac{d U(v)}{d v}+\sqrt{2 \gamma \eta}(t)
$$

with $U(v)=c_{1} v^{2}-c_{2} v|v|^{2}+c_{3} v^{4}$ (with positive $c_{1}, c_{2}$ and $c_{3}$ ). Numerical inspection, clearly indicates that $C_{1}(t), C_{2}(t)$ and $C_{3}(t)$ are practically indistinguishable.

A simple condition produces the observed behavior. In fact, the time correlation of $v(t)$ with a generic function of the initial velocity $f[v(0)]$ can be written as

$$
\begin{array}{r}
\langle v(t) f[v(0)]\rangle=\int d v_{t} \int d v_{0} p_{v}\left(v_{0}\right) \mathcal{P}_{t}\left(v_{t} \mid v_{0}\right) v_{t} f\left[v_{0}\right]= \\
\int d v_{0} p_{v}\left(v_{0}\right) f\left[v_{0}\right]\left\langle v_{t} \mid v_{0}\right\rangle,
\end{array}
$$

where $\mathcal{P}_{t}\left(v_{t} \mid v_{0}\right)$ is the conditional probability of observing $v(t)=v_{t}$ if $v(0)=v_{0}$ (time translation invariance is assumed) and $\left\langle v_{t} \mid v_{0}\right\rangle=\int d v_{t} \mathcal{P}_{t}\left(v_{t} \mid v_{0}\right) v_{t}$ is the average of $v(t)$ conditioned to $v(0)=v_{0}$.

If, for some reason, the dependence on time and on $v(0)$ can be factorized, i.e. $\left\langle v_{t} \mid v_{0}\right\rangle=g(t) q\left(v_{0}\right)$, with $g$ and $q$ two generic functions, then the time behavior results independent of the choice of the function $f(v)$, i.e. of the order of the correlation. This happens in model (iii), where in spite of the non-Gaussian shape of the velocity pdf, the equivalence between $R(t)$ and $C_{1}(t)$, as well as any other correlation $C_{f}(t)=\langle v(t) f[v(0)]\rangle /\langle v(0) f[v(0)]\rangle=$ $R(t)=\exp \left(-\frac{r(r+1)}{4} t\right)$, with any generic function $f$ of the initial velocity value, is exact for the case $d=2$ and [117] for $d=1$, see Appendix A. 
Let us discuss further experiments, with different kinds of perturbations, which involve more than one particle. A classical procedure, to avoid the heating of the system when a global perturbation is applied, is the following [44]: once a steady-state has been reached, the system is perturbed impulsively at time 0 by a force applied (non-uniformly) on every particle. The response is then monitored in time. The force acting on particle $i$ is

$$
\mathbf{F}\left(\mathbf{r}_{i}, t\right)=\zeta_{i} \boldsymbol{\xi}\left(\mathbf{r}_{i}, t\right)
$$

with the properties $\boldsymbol{\nabla} \times \boldsymbol{\xi} \neq 0, \boldsymbol{\nabla} \cdot \boldsymbol{\xi}=0$, where $\zeta_{i}$ is a particle dependent variable with randomly assigned \pm 1 values. A simple case is realized by a transverse perturbation $\xi(\mathbf{r}, t)=\left(0, \xi \cos \left(k_{x} x\right) \delta(t)\right)$, where $k_{x}$ is compatible with the periodic boundary conditions, i.e. $k_{x}=2 \pi n_{k} / L_{x}$ with $n_{k}$ integer and $L_{x}$ the linear horizontal box size. The staggered response function (i.e. the current induced at $t$ by the perturbation at time 0 ), and the conjugate correlation,

$$
\begin{aligned}
R(t) & =\frac{1}{\xi}\left\langle\sum_{i} \zeta_{i} \dot{y}_{i}(t) \cos \left(k_{x} x_{i}(t)\right)\right\rangle, \\
C(t) & =\left\langle\sum_{i} \dot{y}_{i}(t) \dot{y}_{i}(0) \cos \left\{k_{x}\left[x_{i}(t)-x_{i}(0)\right]\right\}\right\rangle
\end{aligned}
$$

are related, at equilibrium, by the Kubo relation $R\left(t, t_{0}\right)=\frac{\beta}{2} C\left(t, t_{0}\right), T_{b}=$ $1 / \beta$ being the bath temperature. It is possible to derive the same relation from Eq. (3.9) with the assumption of uncorrelated variables and Gaussian velocities, and replacing $T_{b}$ with $T_{g}$ of the perturbed species. In Figure 9 , the results of such numerical experiment are shown in a parametrized form. The previous scenario is recovered: the Kubo relation is satisfied with $T_{g}$ replacing $T_{b}$.

\section{The binary mixture and the granular thermometer}

In the binary mixture case, one analyzes a system made of two different components with $N_{1}$ and $N_{2}$ particles, with masses $m_{1}$ and $m_{2}$ respectively, coefficients of restitution $\alpha_{11}$ and $\alpha_{22}$ for collisions among particles of the same species and $\alpha_{12}$ for collisions among particles of different species. Simulations as well as experiments and analytical calculations have shown that, in this case, energy equipartition is broken, i.e. $T_{1} \neq T_{2} \neq T_{b}[127,128,129,130,131]$. At the level of Boltzmann kinetic equation, the temperature ratio of a binary granular mixture subject to stochastic driving of the form given above has been obtained in [130] for the case $\gamma_{s_{i}}=0$ and in [127] for $\gamma_{s_{i}} \neq 0$. In the

case $\gamma_{s_{i}} \neq 0$ a bath temperature can still be defined as $T_{b}=\Gamma_{s_{i}} \gamma_{s_{i}}$. Note that in general $\Gamma_{s_{i}}$ and $\gamma_{s_{i}}$ depend upon $m_{i}$ and the correct elastic limit (i.e. equipartition) is recovered if and only if $T_{b}$ does not depend on $m_{i}$. In [127] it has been shown that a model with $\Gamma_{s_{i}} \propto \sqrt{m_{s_{i}}}$ and $\gamma_{s_{i}} \propto m_{s_{i}}$ fairly repro- 


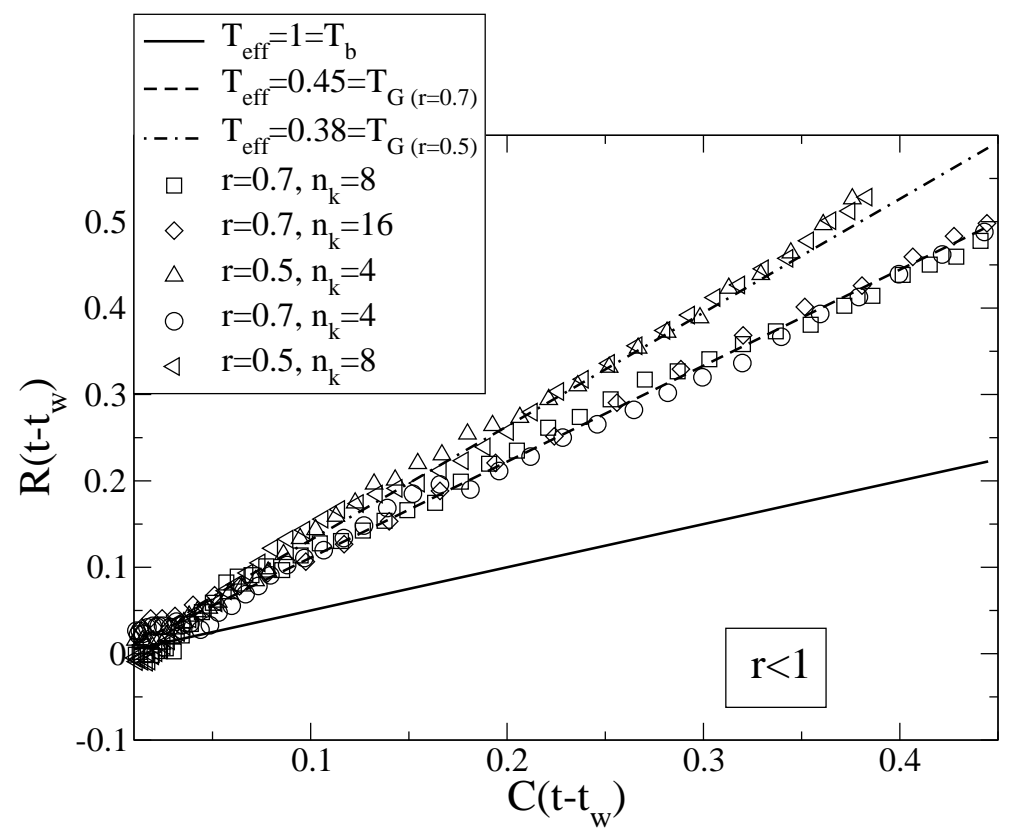

Fig. 9. Parametric plot of $R\left(t-t_{w}\right)$ vs. $C\left(t-t_{w}\right)$ for the numerical experiment with recipe $I I$ (impulsive shear perturbation) with $r<1$, with heating bath, and for different choices of the wave number $n_{k}$ of the perturbation. $T_{b}=1$ and $\tau_{b}=10$, $N=500, \tau_{c}=1, \Xi=0.01, n_{k}=8$, with averages over 10000 realizations, using $t_{w}=100$.

duces experimental results for the temperature ratio $T_{1} / T_{2}$ measured in a gas of grains in a box vertically vibrated. It is also known that equipartition is not recovered even in the so-called tracer limit [132], i.e. in the case $N_{2}=1$ and $N_{1} \gg 1$. For binary mixtures we discuss recent results [116] obtained for both molecular dynamics and DSMC. The above Fluctuation-Response experiment with a small perturbation introduced on a particle can be performed integrating in time, measuring the mobility of a tracer particle and comparing it with its self-diffusion coefficient: this is useful to reduce noise in the measurement of the response function. The mobility of the tracer particle can be measured by applying a small constant drag force $f_{x}$, in the $x$ direction for instance to a given particle, labeled 0 , for times $t>0$ (we assume the system to be isotropic). The linearity of the response is always checked by changing the amplitude of the perturbation. The perturbed particle will reach at large times a constant velocity $\mu f_{x}$, related to the instantaneous response of Eq. (4.59) by

$$
\mu=\frac{1}{m_{0}} \int_{0}^{t} R\left(t^{\prime}\right) d t^{\prime}
$$

The mean-square displacement averaged over many unperturbed dynamics $B(t)=\left\langle\left|\mathbf{r}_{0}(t)-\mathbf{r}_{0}(0)\right|^{2}\right\rangle$ asymptotically grows linearly in time, $B(t) \sim 4 D t$ (in dimension $d=2$ ) with a self-diffusion coefficient given by $D=\int_{0}^{\infty}\langle v(t) v(0)\rangle$. Integrating the relation (4.61), one obtains a prediction for the mobility in the 
uncorrelated Gaussian case:

$$
\mu=\frac{1}{T_{s}} D
$$

where $T_{s}$ is the granular temperature of the species that the perturbed particle belongs to.

The general result for a binary mixture [116] is analogous to the one for monodisperse gases: even when the velocity pdf is non Gaussian, the closeness between correlations of different order makes relation (4.70) true. By using as test particle a particle with index 1 (2) for species 1 (2), one obtains the two responses $\chi_{1}=\overline{x_{1}(t)-x_{1}(0)} \simeq\left\langle\delta v_{1}\right\rangle_{\infty} t$ and $\chi_{2}=\overline{x_{2}(t)-x_{2}(0)} \simeq\left\langle\delta v_{2}\right\rangle_{\infty} t$, and thus the mobilities $\mu_{1}$ and $\mu_{2}$. Two independent Einstein relations $\left(\mu_{i}=D_{i} / T_{i}\right)$ are verified, by plotting $\chi_{i}$ vs. $B_{i}$. In figure 10 we show, as an example, the verification of the validity of Green-Kubo relations using DSMC in spatially homogeneous regime. All the experiments, performed varying the restitution coefficients and the masses of the two components, and with different models and algorithms (homogeneous and non-homogeneous, DSMC and MD) showed identical results, i.e the linearity of response-perturbation relation with effective temperature equal to the granular temperature of the perturbed species. The same is true for other kinds of perturbation, such as the current-shear perturbation experiment discussed above.

In figure 11 an even more striking result is portrayed: the mobility-diffusion parametric graph is shown in the case of a single tracer with different properties with respect to a bulk gas $\left(N_{1}=500, N_{2}=1\right)$. In this case the tracer does not perturb significantly the bulk. However the temperature of the tracer is quite different from the bath temperature as well as from the gas temperature [132]. Again, the effective temperature of the tracer corresponds to its temperature and not to the temperature of the bath or of the bulk. That is to say that a non-perturbing thermometer, used to measure temperature of a granular gas through Fluctuation-Response relations, would measure its own temperature and not the bulk temperature. This is a consequence of the lack of thermalization, or equipartition, due to energy dissipating collisions which characterize stationary states of granular systems.

\section{Failure of the Einstein relation: correlated degrees of freedom}

The factorization of the invariant phase space measure, Eq. (4.60), is no more obvious in model (i) when density increases. Correlations between different degrees of freedom (d.o.f.), that is positions and velocities of the same or of different particles, appear also in homogeneously driven granular gases, as an effect of the inelastic collisions that act similarly to an attractive potential. Such a phenomenon has been discussed for this model of bath in $[133,134,135,136]$ and for other homogeneous thermostats in $[137,138,139]$. In $[133,134]$ it was also discussed the interplay between local density and local granular temperature,

which in some very dissipative cases present strong fluctuations correlated to 

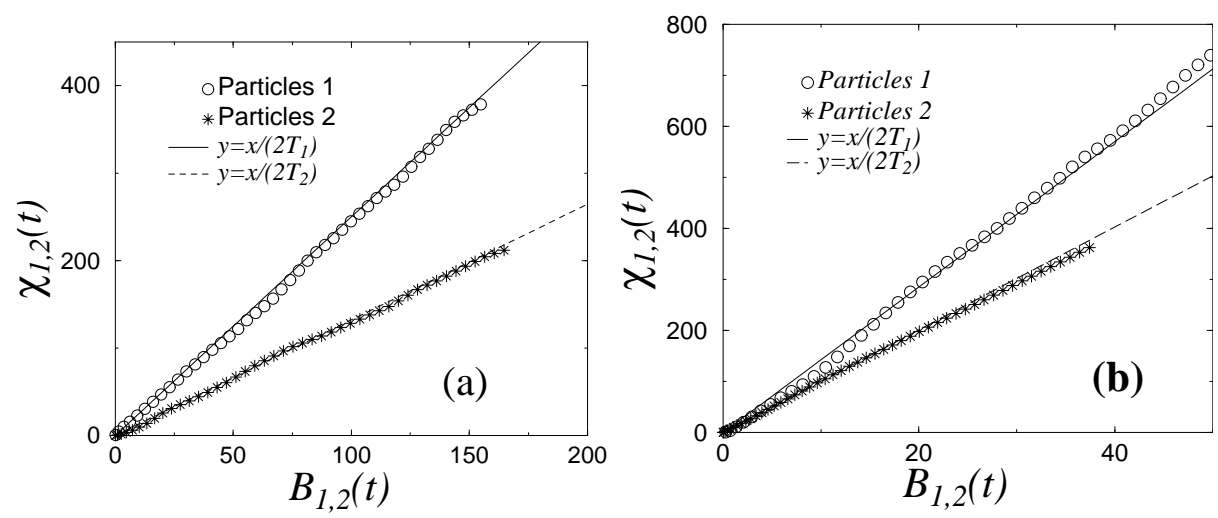

Fig. 10. Binary mixture, homogeneous DSMC: mobility vs. mean-square displacement; left: $\alpha_{11}=0.3, \alpha_{12}=0.5, \alpha_{22}=0.7, m_{2}=3 m_{1}, T_{1} \approx 0.2, T_{2} \approx 0.38$; right: $\alpha_{11}=\alpha_{12}=\alpha_{22}=0.9, m_{2}=5 m_{1}, T_{1} \approx 0.035, T_{2} \approx 0.05$. Symbols are numerical data, lines have slope $1 /\left(2 T_{1}\right)$ and $1 /\left(2 T_{2}\right)$.

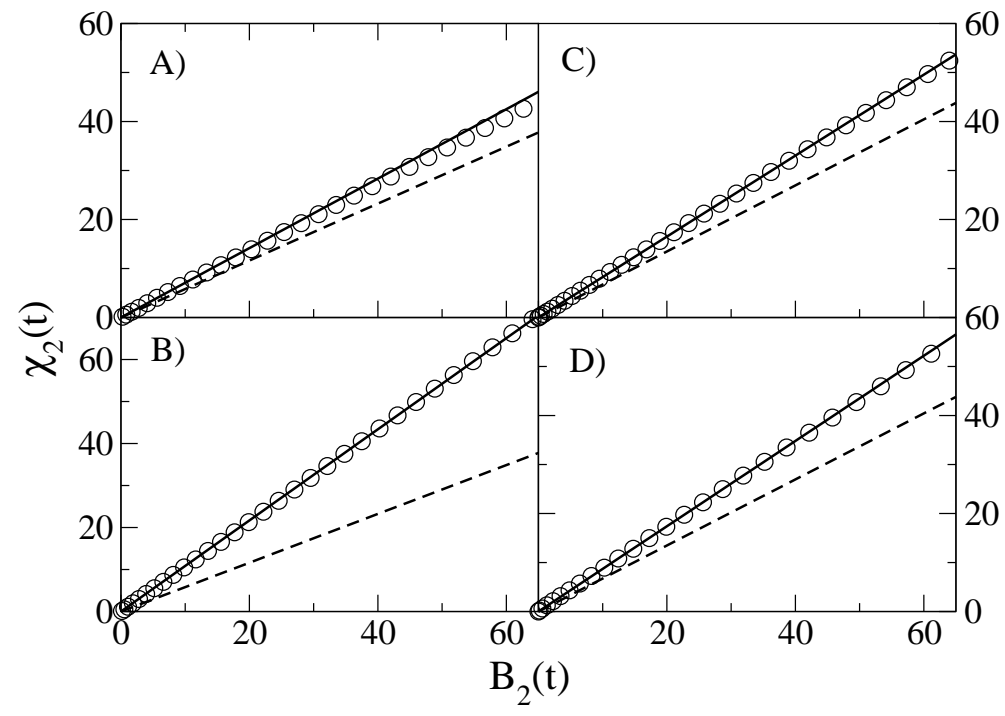

Fig. 11. Binary mixture, homogeneous DSMC: mobility Vs. diffusion of a single particle of mass $m_{\text {tracer }}$ in contact with $N=500$ particles of mass $m$, immersed in a heat bath (i.e. random kicks plus viscosity). We use the following conventions: $\alpha_{\text {tracer }}=\alpha_{12}$ and $\alpha=\alpha_{11}$. Only in case $\mathbf{A}$ ) the tracer is also in contact with the external driving heat bath. A) $m_{\text {tracer }}=m, \alpha=0.9, \alpha_{\text {tracer }}=0.4, T_{g}=0.86$, $\left.T_{g}^{\text {tracer }}=0.70 ; \mathbf{B}\right) m_{\text {tracer }}=m, \alpha=0.9, \alpha_{\text {tracer }}=0.4, T_{g}=0.86, T_{g}^{\text {tracer }}=0.46$; C) $\left.m_{\text {tracer }}=7 \mathrm{~m}, \alpha=\alpha_{\text {tracer }}=0.7, T_{g}=0.74, T_{g}^{\text {tracer }}=0.60 ; \mathbf{D}\right) m_{\text {tracer }}=4 m$, $\alpha=\alpha_{\text {tracer }}=0.7, T_{g}=0.74, T_{g}^{\text {tracer }}=0.57$. The solid line has slope $T_{g}^{\text {tracer }}$, the dashed line has slope $T_{g}$.

each other. These correlations indicate a breakdown of the factorization of the invariant measure, in particular the velocity and the position distributions of a given particle do not factorize. In general, even when relation (4.60) does not hold, one can define (and compute) the marginal probability density function of the component $x$ of the velocity of one particle $i$, as the marginalized of 

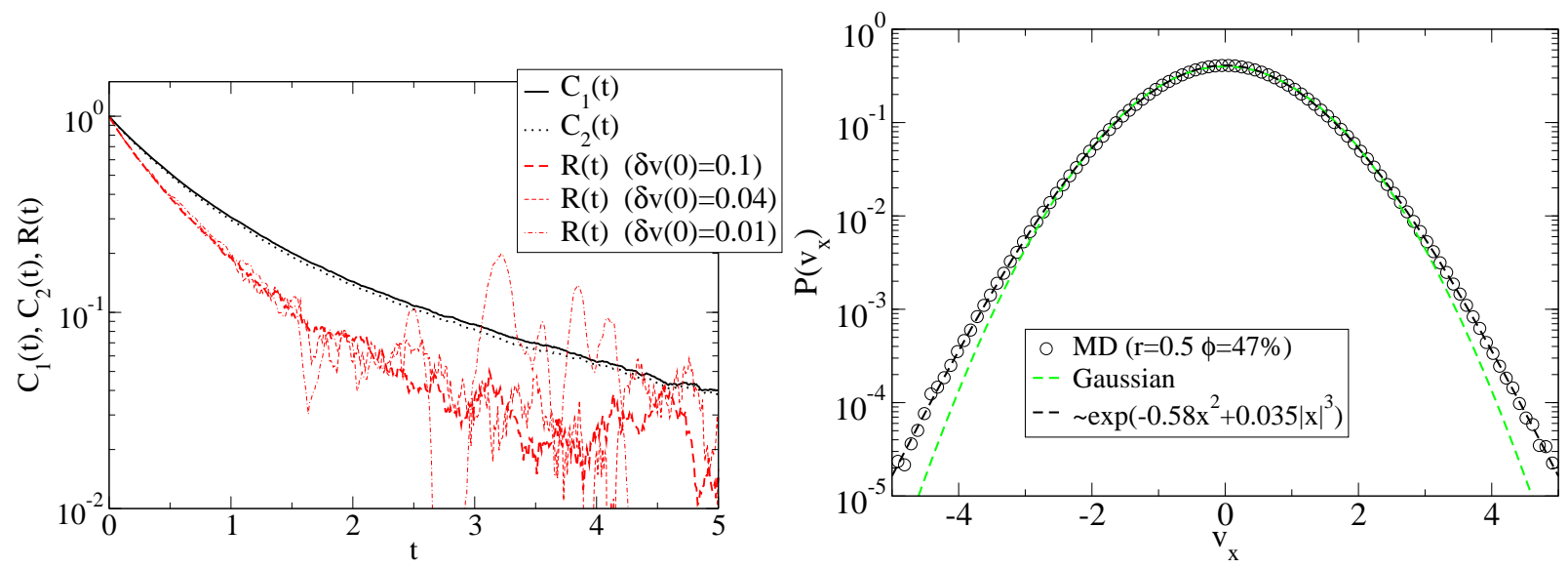

Fig. 12. Left: correlation functions and response. Right: velocity pdf. Both plots refer to the case of a dense granular gas

$\rho\left(\left\{\mathbf{v}_{i}, \mathbf{x}_{i}\right\}\right)$

$$
p_{v}^{(i)}\left(v_{x}\right)=\int \prod_{k=1}^{N} d \mathbf{x}_{i} \prod_{k=1, k \neq i}^{N} d \mathbf{v}_{k} d v_{i}^{y} \rho(\{\mathbf{v}, \mathbf{x}\}) .
$$

However not always does this function play a direct role in the response function. For example, perturbing the $x$ component of the velocity of the $i$-th particle and measuring the response of the same component, one obtains

$$
R(t)=-\left\langle\left. v_{i}^{x}(t) \frac{\partial \ln \rho(\{\mathbf{v}, \mathbf{x}\})}{\partial v_{i}^{x}}\right|_{t=0}\right\rangle \neq-\left\langle\left. v_{i}^{x}(t) \frac{\partial \ln p_{v}^{(i)}\left(v_{i}^{x}\right)}{\partial v_{i}^{x}}\right|_{t=0}\right\rangle .
$$

This is exactly what happens in model (i) (here we discuss only the monodisperse case [118]) when density is increased. In Figure 12, left frame, the correlation functions $C_{1}(t)$ and $C_{2}(t)$ are shown, together with the response function measured with different values of the perturbation $\delta v(0)$. The very good agreement between different response functions guarantees that the system is indeed linearly perturbed. At the same time, the different correlation functions $C_{k}(t)$ are very close, reproducing the phenomenology already observed in the previous dilute cases, with the difference that the time dependence is not exponential but slower, closer to a stretched exponential $\sim \exp \left(-(t / \tau)^{\alpha}\right)$ with $\alpha<1$. Finally, looking at the velocity pdf of the gas, the previously proposed exponential of a cubic expression, Eq. (4.62) with a negligible $c_{3}$ coefficient, is found to perfectly fit the numerical results. Therefore, if the correlations among the different d.o.f. are neglected, using equation (4.63) and the proportionality of the functions $C_{n}(t)$, a verification of the Einstein formula $R(t) \equiv C_{1}(t)$ is still expected. The results displayed in Figure 12, left frame, demonstrate that this is not the case: the hypothesis of weak correlations among different d.o.f. is not appropriate and the correct response function is given by Eq. (4.72). 


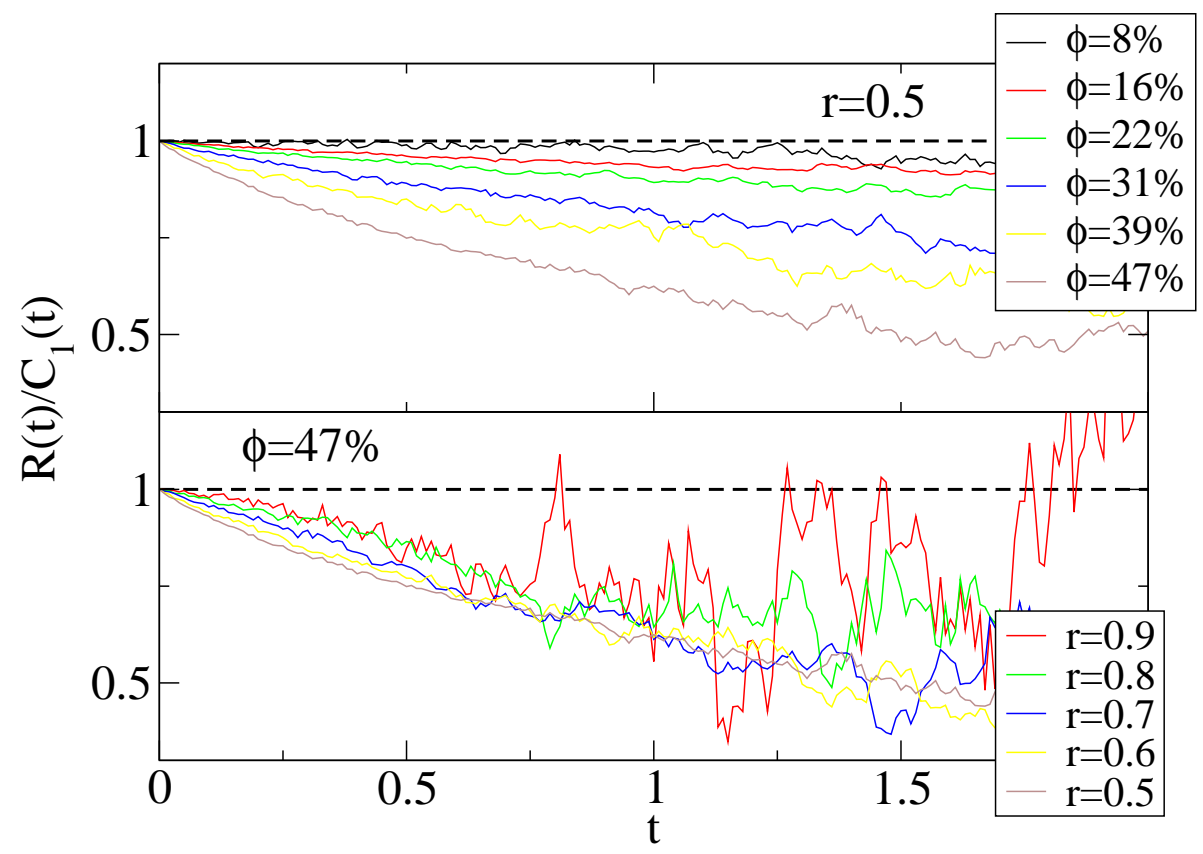

Fig. 13. Ratio between response and auto-correlation for different densities and restitution coefficients

The degree of violation of the Einstein formula increases with the volume fraction $\phi$ and the inelasticity $1-r$, as shown in Figure 13, where the ratio $R(t) / C_{1}(t)$ is reported as a function of time. This observation is consistent with the above argument: correlations among different d.o.f. increase when the probability of repeated contacts (the so-called "ring collisions") is enhanced, and this happens when the excluded volume is reduced as well as when the post-collisional relative velocity is reduced.

\section{A linear model with two correlated variables}

It is interesting to discuss a simple model with only two d.o.f. with Gaussian marginalized pdfs. The two variables, $x, y$, obey the following system of coupled Langevin equations:

$$
\begin{aligned}
& \frac{d x(t)}{d t}=m_{11} x(t)+m_{12} v(t)+\sigma_{11} \eta_{1}(t)+\sigma_{12} \eta_{2}(t) \\
& \frac{d v(t)}{d t}=m_{21} x(t)+m_{22} v(t)+\sigma_{21} \eta_{1}(t)+\sigma_{22} \eta_{2}(t)
\end{aligned}
$$

When the matrices $\hat{m}$ and $\hat{\sigma}$ are diagonal, the two variables are independent. Otherwise, they are correlated. Provided that the matrix $\hat{m}$ is negative definite and $\operatorname{det} \hat{\sigma} \neq 0$, the pdf of the two variables relaxes toward an invariant joint pdf, given by a bivariate Gaussian function. Instead of discussing the general 


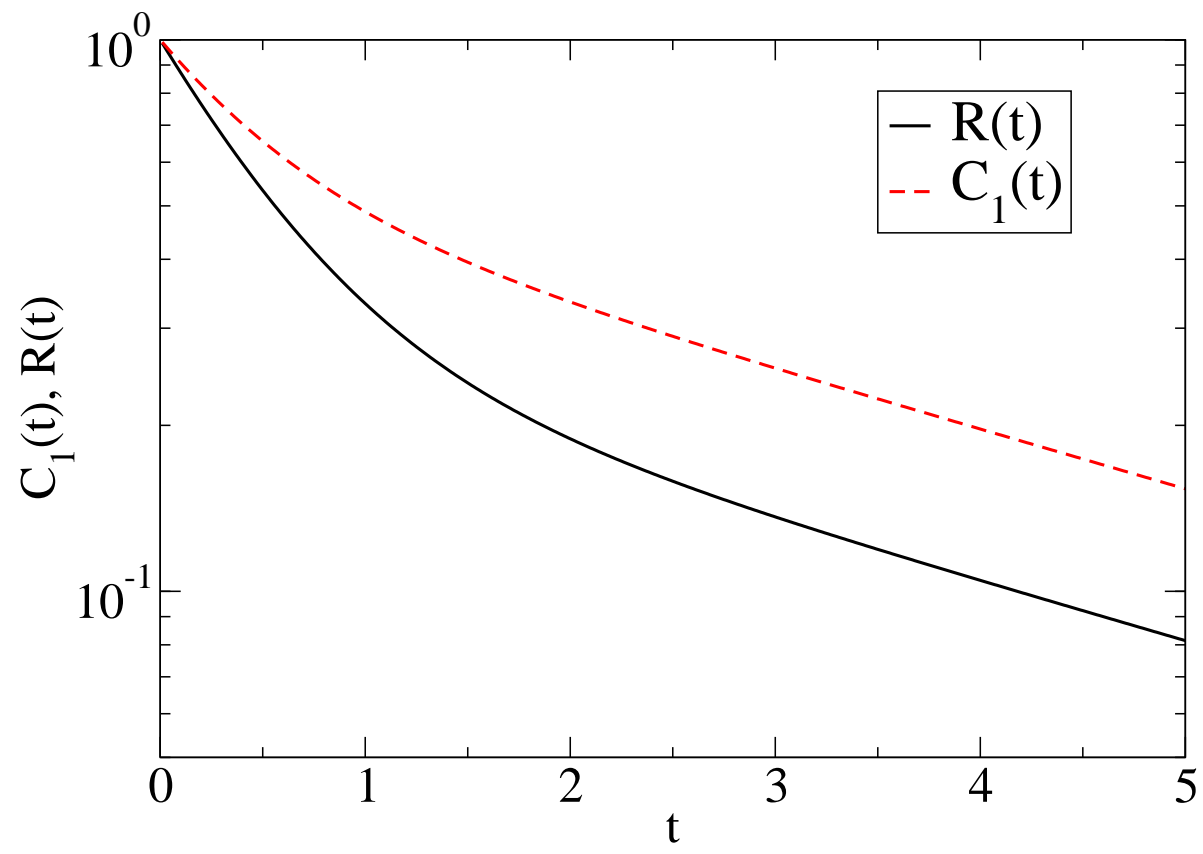

Fig. 14. Response $R(t)$ and velocity correlation $C_{1}(t)$ in the simple Langevin model with correlated variables discussed in Eq. (4.73), with parameters $m_{11}=-1.1$, $m_{12}=m_{21}=0.8, m_{22}=-1$.

case, we suppose that the invariant joint pdf is expressed by

$$
\rho(x, v) \propto \exp \left(-\frac{x^{2}}{2}-\frac{v^{2}}{2}+\frac{x v}{2}\right)
$$

The marginal pdf of each variable is then a Gaussian. Neglecting the correlation among $x$ and $v$, the response of $v$ to a perturbation, would again be expected to be equal to $C_{1}(t)=\langle v(t) v(0)\rangle /\left\langle v^{2}\right\rangle$. On the contrary, the correct response is given through the full formula (3.9), applied to the joint pdf (4.75). The result is

$$
R(t)=\langle v(t) v(0)\rangle-\frac{1}{2}\langle v(t) x(0)\rangle
$$

The difference between the Einstein formula and the correct response is shown in Figure 14 for one choice of the matrix $\hat{m}$.

The role of spatial inhomogeneity

Inspired by a work about violations of the Einstein relation in a non equilibrium model [140], it is natural to conjecture an effective spatial dependence of the pdf of the velocity component for a particle at position $\mathbf{x}$, at time $t$ of 


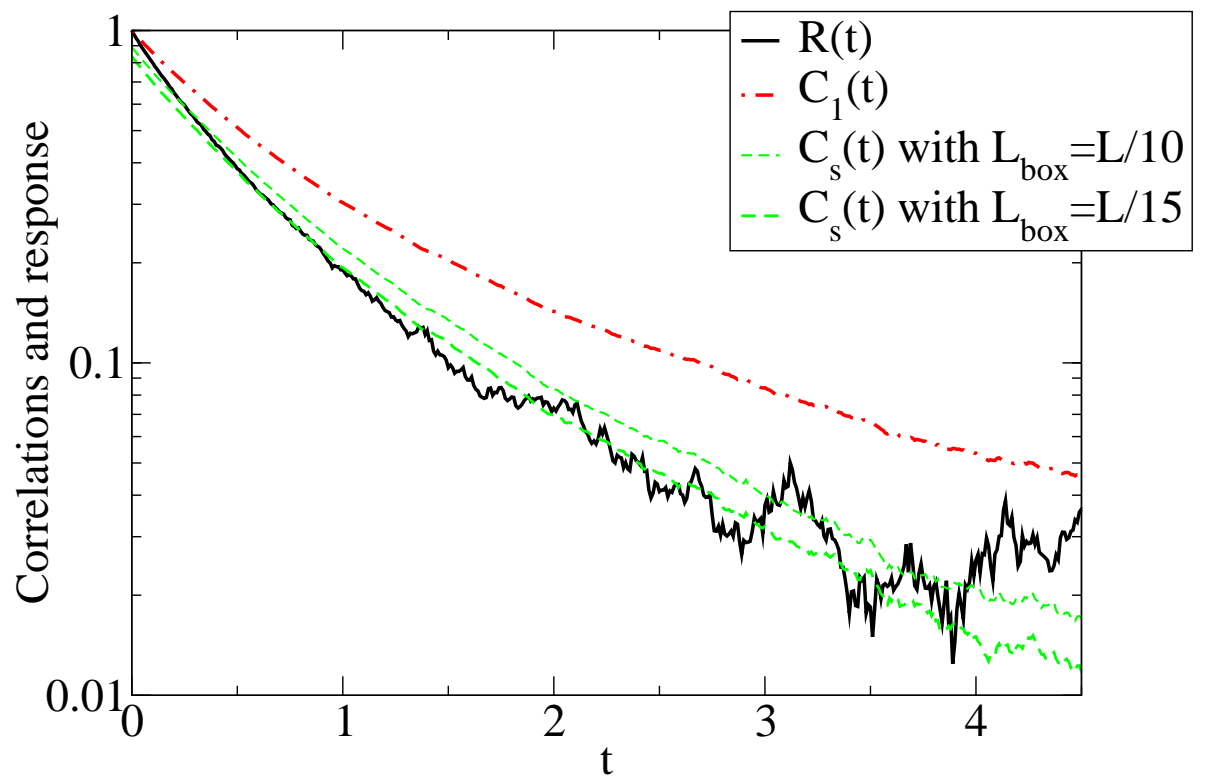

Fig. 15. Response $R(t)$ and different correlation functions for the same MD simulation discussed in Figure 12. The normalized velocity self-correlations $C_{1}(t)$, as well as the correlation $C_{s}(t)$ defined in Eq. (4.78), for different values of the coarse graining radius $L_{b o x}$ are reported.

the form

$$
p_{v}(v, \mathbf{x}, t) \sim \exp \left\{-\frac{[v-u(\mathbf{x}, t)]^{2}}{2 T_{g}}\right\}
$$

where $u(\mathbf{x}, t)$ is a local velocity average, defined on a small cell of diameter $L_{b o x}$ centered in the particle. Such a hypothesis is motivated by the fact that, at high density or inelasticities, spatially structured velocity fluctuations appear in the system for some time, even in the presence of external noise [138,139]. Following relation (3.9), with the ansatz (4.77), a formula for the response function follows:

$$
R(t)=C_{s}=\frac{1}{T_{g}}\langle v(t)\{v(0)-u[\mathbf{x}(0)]\}\rangle
$$

Figure 15 shows that relation (4.78) is fairly verified. Note however that the proposed form (4.77) cannot be completely exact, a spatial dependence of $T_{g}$ should also be included. 


\subsection{Further applications}

\subsubsection{Nano-systems}

Since the early 60's, technology follows a trend towards miniaturization, which has led to the construction of devices of the size of a few tens of nanometers, or smaller. In some circumstances, these devices are built by assembling single molecule after single molecule. While technology at the $\mu m$ scale is known to be more sophisticated, but not substantially different from the macroscopic world technology, it is universally believed that nanotechnology has to face physical features which are quite different from those of the macroscopic world, especially when it comes to devices whose parts are made of an order $O(1)$ up to $O\left(10^{3}\right)$ atoms or molecules. In itself, this does not look particularly surprising. Indeed, macroscopic bodies owe their properties to the very large number of their microscopic constituents, and to the fact that these constituents interact with each other, in very precise fashions. This allows the onset of the state of local thermodynamic equilibrium (when the driving forces are not too large) and of its consequences, like the validity of the hydrodynamic laws. The fact that the microscopic constituents of small systems, like nano-devices, obey the same dynamical laws as those of the constituents of a macroscopic body is not sufficient for the small systems to behave as macroscopic objects. Landau and Lifshitz express this concept as follows [141]:

"At first sight, one may conclude that the growth of the number of particles makes infinitely complicated and intricate the properties of the mechanical system at hand, so much that it becomes impossible to discover any guiding law in the behaviour of the resulting macroscopic body. But this is not the case and, as we will see below, new and very special laws emerge for a very large number of particles.

These are the so-called statistical laws which are due precisely to the exceedingly large number of particles of which the body is made. These laws are in no way reducible to mechanical laws, their specific characteristic is that they make no sense for mechanical systems of a small number of degrees of freedom. Therefore, the presence of many degrees of freedom implies substantially different laws, even though the motion of a system with many degrees of freedom follows the same mechanical laws of a system of a few degrees of freedom."

In particular, the hydrodynamic laws exclude that macroscopic observables may spontaneously fluctuate: this is considered possible, but practically irrelevant, in many-particles systems. Differently, fluctuations are not negligible in

small systems or in small parts of macroscopic systems, where they might be directly observable $[142,143]$.

Nano-technological devices are then expected to behave differently from ther- 
modynamic systems. Among them, we find, for instance, quantum dots, carbon nano-tubes and molecular machines, whose sizes range from a few to $O(100)$ nanometers. The question is: which are the laws that describe the behaviour of such small systems? This question is particularly important not only for its nano-technological implications, see e.g. Ref.[144], but also for understanding from a fundamental point of view the behaviour of microscopic systems in general and, in particular, biological systems. Indeed, bacteria and cells are quite larger than the few tens of $\mathrm{nm}$ which qualify nanosystems, but their behaviour is determined by events that occur at the nanoscale, like the transport of matter through the cell's membrane.

At the moment there is no clear answer to the above question. However, a widely shared view is that the mechanical laws, which are overshadowed by the statistical laws in macroscopic systems, should play an important role in nanosystems, hence it appears promising to explore the nano-world from a dynamical systems point of view. In particular, the fluctuation relations which are the object of Section 5 are expected to be applicable to nano-phenomena. Indeed they have been tested and verified to various extents [142,143], as discussed in Section 5. The study of granular systems, in turn, may provide insights on the above questions, since they would be mesoscopic objects, were their grains of the size of atoms or molecules.

\subsubsection{Fluctuations and response in biological systems}

Some applications of linear response theory to biological systems have been recently discussed by Kaneko and co-workers $[145,146]$. The idea is that fluctuations, always present in living organisms, are relevant for the response of average properties to changes in the environment. Environmental conditions can be seen as parameters determining the distribution of fluctuations of measurable quantities: for example, the concentration of a protein in a cell, as well as its fluctuations, are influenced by many parameters, such as temperature, $\mathrm{pH}$ value and so on. When an external perturbation is switched on, the parameters change and the distributions of biological variables respond in some way. A general relation for this response can be derived: in the following, for simplicity but without loss of generality, we assume that the distribution of fluctuations can be approximated by a Gaussian, and we discuss the case of a scalar external parameter $a$ and a unique scalar observable $x$. Upon a change $\Delta a$ of the parameter, the distribution of $x$ changes from $\rho_{a}(x)$ to $\rho_{a+\Delta a}(x)$, which can be written as

$$
\rho_{a+\Delta a}(x)=\rho_{a}(x) \mathrm{e}^{\ln \rho_{a+\Delta a}(x)-\ln \rho_{a}(x)}=\rho_{a}(x) \mathrm{e}^{\epsilon(a, \Delta a, x)} .
$$


The Gaussian assumption allows us to neglect the powers of $x$ higher than $x^{2}$ in the expansion of $\epsilon(a, \Delta a, x)=\ln \rho_{a+\Delta a}(x)-\ln \rho_{a}(x)$, obtaining

$$
\rho_{a+\Delta a}(x)=\rho_{a}(x) N \mathrm{e}^{\epsilon^{(1)}(a, \Delta a)\left(x-\langle x\rangle_{a}\right)+\frac{1}{2} \epsilon^{(2)}(a, \Delta a)\left(x-\langle x\rangle_{a}\right)^{2}},
$$

where $\epsilon^{(n)}=\left.\left(\partial^{n} \epsilon(a, \Delta a, x) / \partial x^{n}\right)\right|_{x=\langle x\rangle_{a}}$ and $N=e^{\epsilon^{(0)}}$. Using this formula to calculate $\langle x\rangle_{a+\Delta a}$, the average of $x$ in the perturbed system, one obtains:

$$
\langle x\rangle_{a+\Delta a}-\langle x\rangle_{a}=\epsilon^{(1)}(a, \Delta a) \sigma_{a}^{2} \simeq b(a) \sigma_{a}^{2} \Delta a
$$

where the first order in the parameter change has been considered, introducing $b(a)=\partial \epsilon^{(1)}(a, \Delta a) /\left.\partial \Delta a\right|_{\Delta a=0}$, and $\sigma_{a}^{2}=\left\langle\left(x-\langle x\rangle_{a}\right)^{2}\right\rangle$. Equation (4.81) is a particular case of FDR, and in fact can be derived from the generalized relation (3.9): it does not require thermal equilibrium, but only the quasi-Gaussian assumption on $\rho(x)$. It must be noted, however, that in relation (4.81) the coefficient $b(a)$ can in principle be related to $\sigma_{a}^{2}$.

An experimental proof of relation (4.81) has been obtained studying the phenotype response to the exertion of an artificial selection pressure over the genotype of a population of E. Coli bacteria. The fluorescence intensity of the Green Fluorescent Protein (GFP) has been used as a marker of the phenotype state $x$. The external force has been identified as the selection pressure, providing a constant rate of variation of the genotype, taken as the parameter $a$. The distribution of fluctuations of the fluorescence intensity $\rho_{a}(x)$ has been measured in successive generations during the application of the external force. To apply the above relation, the DNA sequence of genes in the cell is regarded as a parameter influencing the variables of the cell system, i.e. the phenotype. The measured distribution $\rho_{a}(x)$ provides the average $\langle x\rangle_{a}$ as well as its variance $\sigma_{a}^{2}$. The proposed relation (4.81), with a coefficient $b$ not dependent on $a$, has been validated by these experimental data.

The above experiment probes the relevance of phenotype fluctuations among clones, i.e. organisms with identical genes: these fluctuations are always possible because living cells are of finite size and at finite temperature. In nature, however, genetic diversity is the norm: the parameter $a$ is not single valued in a population, but is distributed around a mean. The variance of phenotype fluctuations is then also induced by genotype variability. It is this variance that was considered by Sir Ronald Fisher in his celebrated fundamental theorem of natural selection [147], which asserts that evolution speed and genetic variance are proportional. Even if the phenotipic variance of the clones, $V_{i p}$, is not equivalent to that of phenotypes in a genetically diverse population, $V_{g}$, they have been shown to be related in [146]. There, an inequality has been derived, stating that

$$
V_{g} \leq V_{i p}
$$

only assuming that the phenotype distribution is single-peaked and not too broad, which is equivalent to require a robustness of the mean phenotype to 
genotype fluctuations. The equality sign in Eq. (4.82) holds in the limit of high evolution speed, just before the appearance of the error catastrophe, where the phenotype distribution becomes too broad and the single-peak assumption fails.

Concluding, we can say that Fisher's theorem is somehow a biological version of a general form of FDR. Let us stress the formal analogy with statistical mechanics, whose small fluctuations of macroscopic variables can actually be detected from the response functions. Analogously, in biology the fluctuations of the genotype variables, which are the corresponding "microscopic" quantities, can be understood from the phenotype features.

\section{Fluctuation relations}

While the theory of fluctuations around equilibrium states can be considered quite satisfactory and complete, the same cannot be said of the nonequilibrium theory. In this context, the 1993 paper by Evans et al. [148], on the fluctuations of the entropy production rate, has been a pioneering attempt towards a unified theory of a wide range of nonequilibrium phenomena [149]. The relation proposed and tested in [148] is nowadays merely called Fluctuation Relation (FR). It constitutes one of the few general exact results, obtained on purely dynamical grounds, for systems almost arbitrarily far from equilibrium, and close to equilibrium it is consistent with the Green-Kubo and Onsager relations. A considerable number of works have been devoted to it, and various authors have derived it in different ways (e.g. $[150,151,152,153])$.

The FR of Ref.[148] reads

$$
\frac{\operatorname{Prob}_{\tau}(A)}{\operatorname{Prob}_{\tau}(-A)}=e^{\tau A}
$$

where $A$ and $-A$ are averages of the normalized power dissipated in a driven system, in a long time $\tau$, and $\operatorname{Prob}_{\tau}( \pm A)$ is the steady state probability of observing values close to $\pm A$. The relation was derived for the following isoenergetic model of a 2-dimensional shearing fluid:

$$
\left\{\begin{array}{l}
\frac{d}{d t} \mathbf{q}_{i}=\frac{\mathbf{p}_{i}}{m}+\gamma y_{i} \hat{\mathbf{x}} \\
\frac{d}{d t} \mathbf{p}_{i}=\mathbf{F}_{i}(\mathbf{q})+\gamma p_{i}^{(y)} \hat{\mathbf{x}}-\alpha_{t h} \mathbf{p}_{i}
\end{array}\right.
$$

where $\Gamma=\left(\mathbf{q}_{1}, \mathbf{p}_{1}, \ldots, \mathbf{q}_{N}, \mathbf{p}_{N}\right)$ represents one point in phase space, $\gamma$ is the

shear rate in the $y$ direction, $\hat{\mathbf{x}}$ is the unit vector in the $x$-direction, and the 
friction term $\alpha_{t h}$ (also called "thermostat") takes the form

$$
\alpha_{t h}(\Gamma)=-\frac{\gamma}{\sum_{i=1}^{N} \mathbf{p}_{i}^{2}} \sum_{i=1}^{N} p_{i}^{(x)} p_{i}^{(y)}
$$

determined by Gauss' principle of least constraint (cf. Subsection 5.3). In this popular molecular dynamics model, the phase space contraction rate, defined by

$$
\Lambda=-\sum_{k} \sum_{i} \frac{\partial \dot{\mathbf{q}}_{i}^{(k)}}{\partial \mathbf{q}_{i}^{(k)}}+\frac{\partial \dot{\mathbf{p}}_{i}^{(k)}}{\partial \mathbf{p}_{i}^{(k)}},
$$

is proportional to the "thermostat" $\alpha_{t h}$, hence to a quantity $\Omega$, which is the irreversible entropy production, or the energy dissipation rate, divided by $\sum \mathbf{p}_{i}^{2}$.

Evans and Searles focused on the fluctuation properties of $\Omega[151,154,155,156,157,158]$, which differs from $\Lambda$ in most cases. They obtained "transient" FRs, which hold under the sole condition of reversibility of the microscopic dynamics. The term transient means that such relations concern the statistics of evolving ensembles of trajectories, rather than that of the steady states. These authors argued that, in the long $\tau$ limit, the same relations describe the steady state fluctuations. We call $\Omega$-FR's these relations, which will be described in subsection 5.3 .2 .

Gallavotti and Cohen provided the mathematical setting for the results of Ref.[148], introducing the Chaotic Hypothesis [150,159,160]:

Chaotic Hypothesis: A reversible many-particle system in a stationary state can be regarded as a transitive Anosov system ( $f$. Appendix $C$ ) for the purpose of computing its macroscopic properties.

The result is a steady state FR for the fluctuations of $\Lambda$, which we call $\Lambda$ FR, and we describe in subsection 5.3.1. Such a strong assumption as the Chaotic Hypothesis raised the question of which models of physical interest are "Anosov-like", since almost none of them is actually Anosov (lack of uniform hyperbolicity being usual). The idea was that the Anosov property, analogously to the Ergodic property, could hold "in practice". This view faces some difficulties which are evidenced and tackled, for example, in Refs.[161,162].

Other relations which concern the statistics of nonequilibrium systems, and have generated much interest, are the Jarzynski and Crooks relations; they belong to the class of transient FRs, and relate free energy differences between equilibrium states to non-equilibrium processes [163], as described in subsection 5.3.3.

The derivation of the FR's for deterministic systems motivated stochastic approaches, beginning with Kurchan's work [164]. Among these studies, Ref.[165] 
has to be singled out since it solved, in a special case, a difficulty of systems with infinite state spaces, which are quite common. The works by Bodineau and Derrida [166], and by Jona-Lasinio and coworkers [167] also treat fluctuations in stochastic systems, but develop different theories, hence will be discussed separately in Section 7.

We do not attempt an exhaustive review of this subject: we focus mainly on the papers which initiated the various research lines. Because entropy production is one of the quantities which are most investigated in this context, we begin recalling basic facts of Irreversible Thermodynamics. After that, we describe the models in which the FRs were first considered.

\subsection{Irreversible entropy production}

The main object of the FR's is the irreversible entropy production, for systems in Local Thermodynamic Equilibrium (LTE), or the energy dissipation rate, more in general.

Irreversible Thermodynamics can be applied to systems that satisfy LTE [26]. Physically, LTE is due to the local exchange of momentum and energy in many particle collisions, leading to a local Maxwell velocity distribution and local thermodynamics. This allows one to assume that the specific (i.e., per unit mass) local entropy $s$ at a position $\mathbf{r}=\left(r_{1}, r_{2}, r_{3}\right)$ at time $t$ is a function of the local specific energy $u$, of the local mass density $n$, and of the local mass fractions $\mathbf{c}=\left\{c_{i}\right\}_{i=1}^{k}$ of the $k$ constituents out of which the system consists, as it is in equilibrium. In other words, LTE implies

$$
s(\mathbf{r}, t)=s[u(\mathbf{r}, t), n(\mathbf{r}, t), \mathbf{c}(\mathbf{r}, t)]
$$

and

$$
T d s=d u+p d v-\sum_{i=1}^{k} \mu_{i} d c_{i}
$$

where $p$ is the pressure, $v=1 / n$ is the specific volum 11 , and $\mu_{i}$ the chemical potential of component $i$. In particular, LTE assumes that Eq.(5.6) remains valid for a mass element followed along its center of mass motion, even if the total system is not in equilibrium, so that one can write:

$$
T D_{t} s=D_{t} u+p D_{t} v-\sum_{i=1}^{k} \mu_{i} D_{t} c_{i}
$$

where, denoting with $\mathbf{v}(\mathbf{x}, t)$ the macroscopic velocity,

$$
D_{t}=\partial_{t}+\mathbf{v} \cdot \nabla_{\mathbf{r}}
$$

\footnotetext{
${ }^{11}$ In other words, $v$ is the volume per unit mass.
} 
is the (barycentric) substantial time derivative [26]. It turns out that LTE holds for a wide variety of transport phenomena.

The hydrodynamical equations are based on LTE, as well as on the local conservation laws for $u, v$ and the $c_{i}$ 's. Assuming local linear laws for their fluxes, one obtains, for instance, Fick's law for tracer diffusion, with local flux $\mathbf{J}:$

$$
\mathbf{J}(\mathbf{r}, t)=-D \nabla_{\mathbf{r}} n(\mathbf{r}, t)
$$

where $\nabla_{\mathbf{r}} n$ is the local density gradient, and $D$ the diffusion coefficient 12 For viscosity, the local flux is given by Newton's law, and is expressed by

$$
J_{i j}(\mathbf{r}, t)=-\eta \frac{\partial u_{j}}{\partial r_{i}}(\mathbf{r}, t)
$$

where $J_{i j}$ is the $i j$-component of the local pressure tensor, giving the flow of the $j$-component of the local momentum of a fluid in the $r_{i}$-direction, sheared by a linear local velocity gradient $\partial u_{j} / \partial r_{i}$, and $\eta$ is the viscosity coefficient. Similarly, one obtains all the other linear laws of Irreversible Thermodynamics. It is worth pointing out that states described by nonlinear laws, for the local quantities mentioned here, may be necessary in the presence of strong external forces and gradients, but this does not mean that LTE is broken: as long as one can properly speak of local thermodynamic quantities, LTE must hold.

Equation (5.7) and the hydrodynamic equations lead then to the entropy balance equation for the local variation of the entropy density:

$$
n(\mathbf{r}, t) D_{t} s(\mathbf{r}, t)=-\operatorname{div} \mathbf{J}_{s}(\mathbf{r}, t)+\sigma_{s}(\mathbf{r}, t)
$$

where $\mathbf{J}_{s}$ is the local entropy flux, and $\sigma_{s} \geq 0$ is the local irreversible entropy production. This is a quantity related to the dissipated energy, hence should not be confused for the mere entropy variation, which takes place even in dissipationless phenomena, and does not need LTE. Indeed, in the linear regime, $\sigma_{s}$ is usually written as $\sum_{i} X_{i} J_{i}$, where $X_{i}$ and $J_{i}$ are conjugate thermodynamic forces and fluxes, respectively.

\subsection{Ergodic hypothesis: the Khinchin's approach}

As in the study of equilibrium phenomena, it is desirable to develop a microscopic interpretation of the macroscopic theory outlined above, in order to extend our understanding beyond the realm of the previous subsection. To connect the theory of irreversible thermodynamics with a microscopic description, in analogy with equilibrium statistical mechanics, models are needed to

\footnotetext{
$\overline{12}$ More in general, $D$ may be a tensor.
} 
assess the various hypothesis and to derive relations of practical interest. Indeed, while the equilibrium theory is solidly based on the Gibbs ensembles and on the Ergodic Hypothesis, the nonequilibrium counterparts of these ensembles and hypotheses are not identified yet, although various proposals have been made in the literature. Before we continue, it is therefore useful to recall how the microscopic description of equilibrium phenomena is based on the Ergodic Hypothesis, following Khinchin's approach [168]. This will suggest possible avenues of investigation, for the case of nonequilibrium phenomena.

Khinchin looked at the ergodic problem from a physical perspective, avoiding the physically scarcely relevant concept of metrical transitivity, implied by the Birkhoff theorem. His general idea stems from the following facts:

a) statistical mechanics concerns systems with a large number of degrees of freedom;

b) the physical observables are not generic (in mathematical sense) functions;

c) it is physically acceptable that ensemble averages do not coincide with time averages, for sets of initial conditions $\Gamma$ of measure tending to zero when $N \rightarrow \infty$.

Khinchin considers systems with a separable Hamiltonian, i.e. systems whose Hamiltonian can be written as

$$
H=\sum_{n=1}^{N} H_{n}\left(\mathbf{q}_{n}, \mathbf{p}_{n}\right)
$$

and a special class of observables (called sum functions) of the form

$$
f(\Gamma)=\sum_{n=1}^{N} f_{n}\left(\mathbf{q}_{n}, \mathbf{p}_{n}\right)
$$

where $f_{n}=O(1)$. The pressure, the kinetic energy and the single-particle distribution function are examples of sum functions.

Denote by \langle\rangle the microcanonical ensemble average, and let $\bar{f}(\Gamma)$ be the time average of the observable $f$, along a trajectory starting at $\Gamma$. Under quite general hypotheses, and without invoking any metrical transitivity, Khinchin showed that the following holds:

$$
\operatorname{Prob}\left(\frac{|\bar{f}-\langle f\rangle|}{|\langle f\rangle|} \geq K_{1} N^{-1 / 4}\right) \leq K_{2} N^{-1 / 4}
$$

where $K_{1}$ and $K_{2}$ are $O(1)$. This means that ensemble averages of sum functions differ more than a given tolerance from time averages, only along trajectories whose initial conditions have vanishing measure in the $N \rightarrow \infty$ limit. Mazur and van der Linden [169] extended this result to systems of particles interacting through short range potentials. 
This shows that the details of the phase space dynamics (i.e. of the microscopic dynamics) are practically irrelevant for a notion of ergodicity that describes physical systems and, indeed, they must be irrelevant for the thermodynamic behaviour to be as common as it is. In Khinchin's theory, the good statistical properties that are required are mainly explained as consequences of the fact that $N$ is very large.

\subsection{Molecular dynamics models}

The models developed in nonequilibrium molecular dynamics (NEMD) have been adopted, in the development of microscopic theories of irreversible thermodynamics, because they are sufficiently simple to be analyzed in detail, and because of their success in describing various properties of nonequilibrium fluids and solids $[17,170]$. It is known that results produced by simulations of these systems are not reliable if quantum mechanical effects are important, if the inter-atomic forces are too complicated or insufficiently known, if the number of particles needs to be too large, or the simulations time too long. But NEMD models are otherwise appropriate in computing quantities such as transport coefficients, and are a valid alternative to experiments. Furthermore, the FRs were first conceived and investigated in one such model. The range of properties of a given physical system which they can describe, however, is not clearly delineated, and care should be used if the property under investigation is directly affected by the fictitious forces which characterize NEMD. The simplest NEMD models are derived from Gauss' principle of least constraint $[171,17]$ :

Gauss Principle (1829): Consider $N$ point particles of mass $m_{1}, \ldots, m_{N}$, subjected to frictionless bilateral constraints $\mathbf{C}_{i}$ and to external forces $\mathbf{F}_{i}$. Among all motions allowed by the constraints, the natural one minimizes the "curvature", defined by

$$
\mathcal{C}=\sum_{i=1}^{N} m_{i}\left(\ddot{\mathbf{q}}_{i}-\frac{\mathbf{F}_{i}}{m_{i}}\right)^{2}=\sum_{i=1}^{N} \frac{1}{m_{i}} \mathbf{C}_{i}^{2} .
$$

In the case of holonomic constraints, the equations of motion are Hamiltonian while non-holonomic constraints lead to non-Hamiltonian equations. The isokinetic $(I K)$ constraint, which fixes the kinetic energy $K=\sum_{i} \mathbf{p}_{i}^{2} / 2 m$, and the isoenergetic $(I E)$ constraint, which fixes the internal energy $H_{0}=K+\Phi^{i n t}$, 
$\Phi^{\text {int }}$ being the interaction potential, yield:

$$
\left\{\begin{array}{l}
\frac{d}{d t} \mathbf{q}_{i}=\frac{\mathbf{p}_{i}}{m} \\
\frac{d}{d t} \mathbf{p}_{i}=\mathbf{F}_{i}^{i n t}(\mathbf{q})+\mathbf{F}_{i}^{e x t}(\mathbf{q})-\alpha_{t h}(\Gamma) \mathbf{p}_{i}
\end{array} i=1, \ldots, N\right.
$$

where $\mathbf{F}_{i}^{\text {int }}(\mathbf{q})$ and $\mathbf{F}_{i}^{\text {ext }}(\mathbf{q})$ respectively denote the interactions among the particles and the external force driving the system. In particular, for $\mathbf{F}_{i}^{e x t}(\mathbf{q})=$ $c_{i} \mathbf{F}_{e}$, where $c_{i}$ is a charge coupling particle $i$ to the external field $\mathbf{F}_{e}$, and $\mathbf{J}=\sum_{i=1}^{N} c_{i}\left(d \mathbf{q}_{i} / d t\right)$,

$$
\begin{array}{ll}
\alpha_{t h}(\Gamma)=\alpha_{I K}(\Gamma)=\frac{1}{2 K}\left(\mathbf{J} \cdot \mathbf{F}_{e}+\sum_{i=1}^{N} \frac{\mathbf{p}_{\mathbf{i}}}{m} \cdot \mathbf{F}_{i}^{\text {int }}\right) & \text { preserves } K \\
\alpha_{t h}(\Gamma)=\alpha_{I E}(\Gamma)=\frac{1}{2 K} \mathbf{J} \cdot \mathbf{F}_{e} & \text { preserves } H_{0} .
\end{array}
$$

In the case of the SLLOD model, defined by Eqs.(5.2), one has:

$$
\alpha_{I K}(\Gamma)=\frac{1}{2 K} \sum_{i=1}^{N}\left(\mathbf{F}_{i}^{i n t} \cdot \mathbf{p}_{i}-\gamma p_{i}^{(x)} p_{i}^{(y)}\right), \quad \alpha_{I E}(\Gamma)=-\frac{\gamma}{2 K} \sum_{i=1}^{N} p_{i}^{(x)} p_{i}^{(y)}
$$

In the above examples, $\alpha_{I E}$ is the energy dissipation rate divided by the kinetic energy which, in local equilibrium, becomes the entropy production rate. Because $\Lambda=-\operatorname{div}(d \Gamma / d t)$ is in turn proportional to $\alpha_{I E}, \Lambda$ can be identified with the entropy production rate. However, real systems in nonequilibrium steady states can hardly be isoenergetic, and this identification appears to be accidental and of too limited validity.

Depending on the physical property to be described, other constraints can be used; e.g. isobaric, isochoric, isoenthalpic, constant stress constraints. The deterministic term $-\alpha_{t h} \mathbf{p}$ is meant to add or remove energy from the system, to balance the action of the driving forces, so that a steady state can be reached [17] 13 . This constraint has no immediate physical meaning; it merely serves the purpose of replacing the many (practically impossible to treat) degrees of freedom of a real thermostat, without appreciably perturbing the properties of interest of the system under investigation. For quantities not affected by how energy is removed from the system, its form is irrelevant and various mechanical constraints will do $[17,170,172]$. The introduction of one or another such constraint in the equations of motion is analogous to the choice of

$\overline{13}$ This form of deterministic "thermostat" is computationally efficient, and makes the equations of motion time reversal invariant [17]. 
one numerical algorithm or another, for the solution of a given mathematical problem: one must choose the constraint or the algorithm which alters as little as possible the quantity to be computed. The introduction of hyperviscosities in the modellization of turbulence is another analogous procedure.

Constant internal energy $H_{0}$ or constant kinetic energy $K$, which prevent the system from indefinitely "heating up", are popular constraints. The resulting systems are not Hamiltonian, but a Hamiltonian structure is not to be expected in systems in nonequilibrium steady states, if the thermostat degrees of freedom are not included [173]. Indeed, a complete $N$-particle model of a system and of its thermostat consists of Hamiltonian equations, which may be written as:

$$
\frac{d}{d t} \Gamma=\frac{d}{d t}\left(\begin{array}{c}
\Gamma_{s} \\
\Gamma_{r}
\end{array}\right)=G(\Gamma)=\left(\begin{array}{c}
G_{s}\left(\Gamma_{s}, \Gamma_{r}\right) \\
G_{r}\left(\Gamma_{s}, \Gamma_{r}\right)
\end{array}\right)
$$

where $\Gamma_{s}=\left\{\mathbf{q}_{i}, \mathbf{p}_{i}\right\}, i=1, \ldots, N_{s}, \Gamma_{r}=\left\{\mathbf{q}_{i}, \mathbf{p}_{i}\right\}, i=N_{s}+1, \ldots, N$, and the subscript $s$ refers to the $N_{s}$ particles of the thermostatted system, while the subscript $r$ refers to the $N_{r}=N-N_{s}$ particles of the reservoir. Moreover, if the time reversed evolution is allowed in phase space, it is also allowed in the projected space. As the reservoirs remove energy from a driven system, on average, the projected dynamics must be dissipative. 14 Something similar happens in NEMD models, hence their non-Hamiltonian nature is not a hindrance, by itself. However, NEMD models are not obtained through the ideal projection procedure, and have been developed for specific practical purposes. Therefore, they must be used with care $[174,63]$.

Another popular deterministic model is the Nosé-Hoover thermostat [17], defined by:

$$
\frac{d \mathbf{q}_{i}}{d t}=\frac{\mathbf{p}_{i}}{m} ; \quad \frac{d \mathbf{p}_{i}}{d t}=\mathbf{F}_{i}^{i n t}+\mathbf{F}_{i}^{e x t}-\xi \mathbf{p}_{i} ; \quad \frac{d \xi}{d t}=\frac{1}{\theta}\left(\frac{K(\mathbf{p})}{K_{0}}-1\right) ;
$$

where $K_{0}$ is the chosen average of the kinetic energy $K(\mathbf{p})$, and $\theta$ is a relaxation time. Normally, particles interact, the dissipation is not too high and the dynamics are sufficiently ergodic that the initial value of $\xi$ does not matter. In the small $\theta$ limit, Nosé-Hoover approximates Gaussian $I K$ dynamics, but is more realistic and, in equilibrium, it generates canonical distributions as appropriate for macroscopic isothermal systems.

The models illustrated above, like many others developed in NEMD, contain artificial forces, which are not present in Nature. The fact that they accurately describe certain properties of real systems, nonetheless, suggests that a form

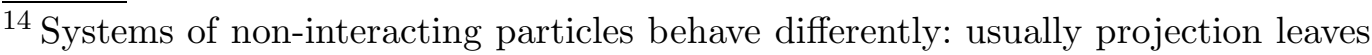
the dynamics Hamiltonian.
} 
of equivalence of ensembles may be envisaged in nonequilibrium statistical mechanics. Several works have been devoted to clarify this issue. The first of them, as far as we know, are due to Evans and Morriss [175,17]. For the equivalence of various thermostatted responses, see Refs.[176,177,172,178,179], while $[178,179]$ show that the phase space dimensionality loss, due to dissipation, is a bulk phenomenon even when the thermostat acts only on the boundaries [180]. Other aspects of the equivalence of deterministic thermostats are discussed in $[181,132]$. In practice, one finds that response to external drivings is not too sensitive to the choice of the thermostatting mechanisms and that, in some cases, the equivalence of behaviors reaches rather subtle dynamical properties $[182,183,174]$.

\subsection{Deterministic systems: transient and steady state relations}

In this Section, we discuss three sets of fluctuation relations, part of which concerns steady states, while the others describe properties of transient states arbitrarily far form equilibrium. The Gallavotti-Cohen and the steady state Evans-Searles approaches lead to steady state relations. The transient EvansSearles, the Jarzynski and the Crooks approaches lead to transient relations.

\subsubsection{The Gallavotti-Cohen approach}

The idea proposed by Gallavotti and Cohen [150], is that dissipative, reversible, transitive Anosov maps (cf. Appendix C) are idealizations of nonequilibrium particle systems, hence that properties like the $\Lambda$-FR are enjoyed by physical systems as well. That the system evolves with discrete or continuous time, was thought to be a side issue in [150], as apparently confirmed by Gentile's derivation for continuous time [184]. The proof of the $\Lambda$-FR for such maps followed these steps. First of all, it is known [185] that one Anosov map $S 15$ admits a special kind of partition of its phase space $\mathcal{M}$, which is called Markovian. This is a subdivision of $\mathcal{M}$ in cells whose interiors are disjoint from each other, and whose boundaries are invariant sets constructed using the stable and unstable manifolds. Consequently, the interior of a cell is mapped by $S$ in the interior of other cells, and not across two cells, which would include a piece of their boundary. Furthermore, arbitrarily fine partitions can be constructed, exploiting the time reversibility of the dynamics. Gallavotti and Cohen further assume that the dynamics is transitive, i.e. that a typical trajectory explores all regions of $\mathcal{M}$, as finely as one wishes. It is this structure that guarantees that probability weights of the kind conjectured in

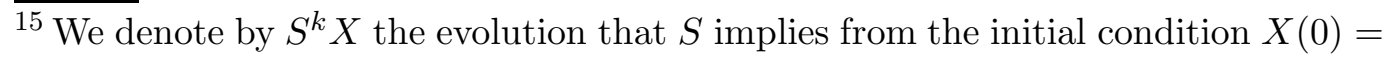
$X$, i.e. $X(1)=S X, X(2)=S^{2} X$, etc. 
[148], cf. Eq.(C.3), from which the $\Lambda$-FR follows, can be assigned to the cells of a finite Markov partition.

More precisely, let $\Lambda(X)=-\log J(X)$, where $J$ is the Jacobian determinant of $S, 16$ and consider the probability (or frequency of observation along one steady state trajectory) of the dimensionless phase space contraction rate, obtained along a trajectory segment $w_{X, \tau}$, of origin $X \in \mathcal{M}$ and duration $\tau$. This quantity is defined by

$$
e_{\tau}(X)=\frac{1}{\tau\langle\Lambda\rangle} \sum_{k=-\tau / 2}^{\tau / 2-1} \Lambda\left(S^{k} X\right)
$$

where $\langle$.$\rangle is the phase space average, with respect to the steady state proba-$ bility distribution. Let $J^{u}$ be the Jacobian determinant of $S$ restricted to the unstable manifold $V^{+}$, i.e. the product of the asymptotic factors of separation of nearby points, along the directions in which distances asymptotically grow at an exponential rate. If the system is Anosov, the probability that $e_{\tau}(X)$ falls in $B_{p, \epsilon}=(p-\epsilon, p+\epsilon)$ coincides, in the limit of fine Markov partitions and long $\tau$ 's, with the sum of the weights

$$
w_{X, \tau}=\prod_{k=-\tau / 2}^{\tau / 2-1} \frac{1}{J^{u}\left(S^{k} X\right)}
$$

of the cells containing the points $X$ such that $e_{\tau}(X) \in B_{p, \epsilon}$. Then, if $\pi_{\tau}\left(B_{p, \epsilon}\right)$ is the corresponding probability, one can write

$$
\pi_{\tau}\left(e_{\tau}(X) \in B_{p, \epsilon}\right) \approx \frac{1}{M} \sum_{X, e_{\tau}(X) \in B_{p, \epsilon}} w_{X, \tau}
$$

where $M$ is a normalization constant. If the support of the physical measure is $\mathcal{M}$, which is the case if the dissipation is not exceedingly high [186], time reversibility guarantees that the support of $\pi_{\tau}$ is an interval $\left[-p^{*}, p^{*}\right], p^{*}>0$, and one can consider the ratio

$$
\frac{\pi_{\tau}\left(B_{p, \epsilon}\right)}{\pi_{\tau}\left(B_{-p, \epsilon}\right)} \approx \frac{\sum_{X, e_{\tau}(X) \in B_{p, \epsilon}} w_{X, \tau}}{\sum_{X, e_{\tau}(X) \in B_{-p, \epsilon}} w_{X, \tau}}
$$

where each $X$ in the numerator has a counterpart in the denominator. Denoting by $I$ the involution which replaces the initial condition of one trajectory

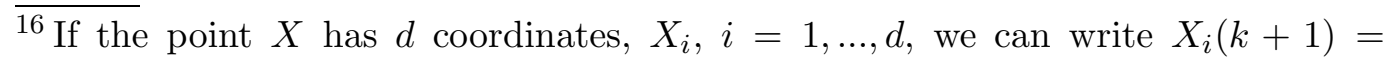
$f_{i}(X(k))$, where $f_{i}$ is a suitable function determined by $S$. Then $J(X)$ is the absolute value of the determinant of the matrix $\left(\partial f_{i} / \partial X_{j}\right)_{X}$. 
with the initial condition of the reversed trajectory, time reversibility 17 yields:

$$
\Lambda(X)=-\Lambda(I X), \quad w_{I X, \tau}=w_{X, \tau}^{-1} \quad \text { and } \quad \frac{w_{X, \tau}}{w_{I X, \tau}}=\exp (\tau\langle\Lambda\rangle p)
$$

if $e_{\tau}(X)=p$. Taking small $\epsilon$ in $B_{p, \epsilon}$, the division of each term in the numerator of (5.20) by its counterpart in the denominator approximately equals $e^{\tau\langle\Lambda\rangle p}$, which then equals the ratio in (5.20). In the limit of small $\epsilon$, infinitely fine Markov partition and large $\tau$ one obtains:

Theorem (Gallavotti-Cohen). Let $(\mathcal{M}, S)$ be dissipative (i.e. $\langle\Lambda\rangle>0)$, reversible and assume that the Chaotic Hypothesis holds. Then,

$$
\frac{\pi_{\tau}\left(B_{p, \epsilon}\right)}{\pi_{\tau}\left(B_{-p, \epsilon}\right)}=e^{\tau\langle\Lambda\rangle p}
$$

with an error in the argument of the exponential which can be estimated to be $p, \tau$ independent.

If the $\Lambda$-FR (hence the Chaotic Hypothesis on which it is based) holds, the function $C(p ; \tau, \epsilon)=(1 / \tau\langle\Lambda\rangle) \log \left[\pi_{\tau}\left(B_{p, \epsilon}\right) / \pi_{\tau}\left(B_{-p, \epsilon}\right)\right]$, tends to a straight line of slope 1 for growing $\tau$, apart from small errors. If $\Lambda$ can be identified with a physical observable, the $\Lambda$-FR is a statement on the physics of nonequilibrium systems.

Under the assumption that $\Lambda$ coincides with the entropy production rate, the $\Lambda$-FR can be used to obtain the Green-Kubo relations and the Onsager reciprocal relations, in the limit of small external drivings [187]. This way, the $\Lambda$-FR appears to be an extension of such relations to nonequilibrium systems. Gallavotti assumes that the (Gaussian isokinetic, continuous time) system is driven by the $\ell$ fields $F=\left(F_{1}, F_{2}, \ldots, F_{\ell}\right)$, that the phase space contraction rate vanishes when all drivings vanish, and that

$$
\Lambda(X)=\sum_{i=1}^{\ell} F_{i} J_{i}^{0}(X)+O\left(F^{2}\right) .
$$

This relation defines the linear "currents" $J_{i}^{0}$, which are proportional to the forces $F_{i}$. Then, the fast decay of the $\Lambda$-autocorrelation function, leads to this expansion for the large deviation functional

$$
\zeta(p)=-\lim _{\tau \rightarrow \infty} \frac{1}{\tau} \log \pi_{\tau}(p)=\frac{\langle\Lambda\rangle^{2}}{2 C_{2}}(p-1)^{2}+O\left((p-1)^{3} F^{3}\right)
$$

$\overline{17}$ Time reversibility means: $I S^{n}=S^{-n} I$, where $I^{2}=$ Identity. For instance, $I$ is the reversal of momenta for Eqs.(5.11), but is more complicated for the shearing system of Ref.[148]. 
where

$$
C_{2}=\int_{-\infty}^{\infty}\left\langle\Lambda\left(S^{t} X\right) \Lambda(X)\right\rangle_{\mathrm{c}} d t
$$

and $\langle.\rangle_{\mathrm{c}}$ denotes the cumulant. Thus, the $\Lambda$-FR, Eq.(5.22), leads to $\langle\Lambda\rangle=$ $C_{2} / 2+O\left(F^{3}\right)$. Now, let the full (nonlinear) "currents" be defined by $J_{i}(X)=$ $\partial_{F_{i}} \Lambda(X)$, and the transport coefficients be $L_{i j}=\left.\partial_{F_{j}}\left\langle J_{i}\right\rangle\right|_{F=0}$. The derivatives with respect to the parameters $F$ require a property of differentiability of SRB measures, which has been proven by Ruelle [188]. Assuming this property, the validity of the $\Lambda$-FR and time reversibility, one can write

$$
\langle\Lambda\rangle=\left.\frac{1}{2} \sum_{i, j=1}^{\ell}\left(\partial_{F_{j}}\left\langle J_{i}\right\rangle+\partial_{F_{i}}\left\langle J_{j}\right\rangle\right)\right|_{F=0} F_{i} F_{j}=\frac{1}{2} \sum_{i, j=1}^{\ell}\left(L_{i j}+L_{j i}\right) F_{i} F_{j}
$$

to second order in the forces. Then, equating this with $C_{2} / 2$ and considering $\left(L_{i j}+L_{j i}\right) / 2$ with $i=j$, one recovers the Green-Kubo relations. To obtain the symmetry $L_{i j}=L_{j i}$, Gallavotti extends the $\Lambda$-FR to consider the joint distribution of $\Lambda$ and its derivatives. He introduces the dimensionless current $q$, averaged over a long time $\tau$, through the relation

$$
\frac{1}{\tau} \int_{-\tau / 2}^{\tau / 2} F_{j} \partial_{F_{j}} \Lambda\left(S^{t} X\right) d t=F_{j}\left\langle\partial_{F_{j}} \Lambda\right\rangle q(X)
$$

and considers the joint distribution $\pi_{\tau}(p, q)$, with corresponding large deviation functional $\zeta(p, q)=-\lim _{\tau \rightarrow \infty} \frac{1}{\tau} \log \pi_{\tau}(p, q)$. The result is a relation similar to the $\Lambda$-FR:

$$
\lim _{\tau \rightarrow \infty} \frac{1}{\tau\langle\Lambda\rangle p} \log \frac{\pi_{\tau}(p, q)}{\pi_{\tau}(-p,-q)}=1 .
$$

This makes the difference $(\zeta(p, q)-\zeta(-p,-q))$ independent of $q$, which leads to the desired result, $L_{i j}=L_{j i}$, in the limit of small $F$. This work was refined in [189]; for related results, based on orbital measures, see Refs.[190,191].

Assuming that the currents and transport coefficients, here defined in terms of the phase space contraction rate, do represent physical quantities, these results show that the FRs are consistent with Irreversible Thermodynamics, close to equilibrium. Hence they may be considered in the construction of a comprehensive nonequilibrium theory. However, some difficulties affect the present approach; the first being that $\Lambda$, which is directly related to the thermostatting term $\alpha_{t h}$, only in very special cases results proportional to the energy dissipation rate divided by the kinetic energy, $\Omega$.

Because global fluctuations are not observable in macroscopic systems, local fluctuation relations have been devised, see e.g. Refs.[192,193,194], and in [183] also a local version the $\Lambda$-FR was tested numerically. In particular, the local $\Lambda$ FR of Ref.[194] concerns an infinite chain of weakly interacting chaotic maps. Let $V_{0}$ be a finite region of the chain centered at the origin, $T_{0}>0$ be a time 
interval, and define

$$
\langle\Lambda\rangle=\lim _{V_{0}, T_{0} \rightarrow \infty} \frac{1}{\left|V_{0}\right| T_{0}} \sum_{j=0}^{T_{0}-1} \Lambda_{V_{0}}\left(S^{j} X\right), \quad p=\frac{1}{\langle\Lambda\rangle|V|} \sum_{j=-T_{0} / 2}^{T_{0} / 2} \Lambda_{V_{0}}\left(S^{j} X\right)
$$

where $V=V_{0} \times T_{0}, \Lambda_{V_{0}}(X)$ is the contribution to $\Lambda$ given by $V_{0}$, and $|E|$ denotes the volume of the set $E$. Then, one obtains:

$$
\pi_{V}(p)=e^{\zeta(p)|V|+O(|\partial V|)}, \quad \text { with } \frac{\zeta(p)-\zeta(-p)}{p\langle\Lambda\rangle}=1 \quad \text { and }|p|<p^{*}
$$

where $|\partial V|$ is the size of the boundary of $V, p^{*} \geq 1$ and $\zeta$ is analytic in $p$. The contribution of the boundary term $|\partial V|$ should decrease with growing $V$, leading to the $\Lambda$-FR in the limit of large (compared to microscopic scales) volume $V_{0}$ and long times $T$.

The problem of local fluctuations, naturally leads to the possibility of extending Onsager-Machlup theory to nonequilibrium systems. This has been done by Gallavotti $[195,196]$, under the assumption that the entropy production rate is proportional to $\Lambda$.

The fact that $\Lambda \neq \Omega$ in general, and the identification of the systems of physical interest which verify the Chaotic Hypothesis, hence the $\Lambda$-FR, pose an interesting question. For instance, Ref.[150] assumed that the $\Lambda$-FR could apply to systems which have singular $\Lambda$, since the $\Lambda$-FR had been proposed and verified in one such system [148]. Later, however, the steady state $\Lambda$-FR was found to be hard, if not impossible, to verify in non-isoenergetic systems with singular $\Lambda$, close to equilibrium $[156,197,198]$. In [161] various scenarios are proposed to explain these facts. One of them concerns Gaussian isokinetic systems, whose $\Lambda$ is the sum of a dissipative term and a conservative term, and may be singular because of the interaction potentials (cf. Eqs. $(5.12,5.14)$ ). In that case, the dissipative term $\Omega$ obeys the FR, while the conservative term does not, but its averages over long time intervals should become negligible with respect to the averages of $\Omega$ as the length of the intervals grow $[198,161]$. Thus, in the long time limit, the $\Lambda$-FR should hold as a consequence of the validity of the $\Omega$-FR, while the convergence times of the $\Lambda$-FR would diverge when equilibrium is approached, because $\Omega$ vanishes as the square of the driving forces. Moreover, for reasons of symmetry, the range $\left[-p^{*}, p^{*}\right]$ of validity of the $\Lambda$-FR must shrink to $\{0\}$ when the driving fields vanish, so that the $\Lambda$-FR may hold but only for trivial fluctuations.

Trying to understand these facts, Refs.[161,199,162] concluded that in some cases $\Lambda$ describes heat fluxes, not entropy productions, hence that in those cases the $\Lambda$-FR has to be modified, to mimic the heat FR of Van Zon and Cohen for stochastic systems [200]. In particular, Ref.[162] considers systems 
of the following form

$$
\frac{d}{d t} \mathbf{q}_{i}=\mathbf{p}_{i} \quad \frac{d}{d t} \mathbf{p}_{i}=E-\partial_{\mathbf{q}_{i}} \Phi-\alpha \mathbf{p}_{i} \quad \Lambda=\Lambda^{(0)}-\beta \frac{d}{d t} V
$$

where $V$ is related to the interaction potential, 18 assuming that they have an equilibrium $(E=0)$ distribution with exponentially decaying tails, while $\Lambda^{(0)}$ has Gaussian tails. It is then assumed that the tails have the same properties when $E \neq 0$. Then, the average of $\Lambda$ in a time $\tau$ takes the form

$$
\bar{\Lambda}_{0, \tau}(X)=\frac{1}{\tau} \int_{0}^{\tau} \Lambda\left(S^{t} X\right) d t=\bar{\Lambda}_{0, \tau}^{(0)}(X)+\frac{\beta}{\tau}\left[V\left(S^{\tau} X\right)-V(X)\right] .
$$

For chaotic systems, and large $\tau$, it is now reasonable to assume that $\Lambda^{(0)}$, $V_{f}(X)=V\left(S^{\tau} X\right)$ and $V_{i}(X)=V(X)$ are independently distributed. This, together with the exponential tails of $V$, leads to

$$
\lim _{\tau \rightarrow \infty} \frac{1}{\tau} \log M\left(p^{*}\right)=\lim _{\tau \rightarrow \infty} \frac{1}{\tau} \log \int_{-p^{*}\langle\Lambda\rangle}^{p^{*}\langle\Lambda\rangle} d \Lambda^{(0)} e^{\tau \tilde{\zeta}_{0}\left(\Lambda^{(0)}\right)-\tau\left|\Lambda-\Lambda^{(0)}\right|}
$$

where

$M\left(p^{*}\right)=\int_{-p^{*}\langle\Lambda\rangle}^{p^{*}\langle\Lambda\rangle} d \Lambda^{(0)} \int_{0}^{\infty} d V_{i} \int_{0}^{\infty} d V_{f} e^{\tau \tilde{\zeta}_{0}\left(\Lambda^{(0)}\right)-\beta\left(V_{i}+V_{f}\right)} \delta\left[\tau\left(\Lambda-\Lambda^{(0)}\right)+\beta\left(V_{i}-V_{f}\right)\right]$

and $\tilde{\zeta}_{0}\left(\Lambda^{(0)}\right)$ is the rate (Cramer) function of $\Lambda^{(0)}$. Then, one obtains

$$
\begin{aligned}
\tilde{\zeta}(\Lambda)=\max _{\Lambda^{(0)} \in\left[-p^{*}\langle\Lambda\rangle, p^{*}\langle\Lambda\rangle\right]}\left[\tilde{\zeta}_{0}\left(\Lambda^{(0)}\right)-\left|\Lambda-\Lambda^{(0)}\right|\right]= \\
= \begin{cases}\tilde{\zeta}_{0}\left(\Lambda_{-}\right)-\Lambda_{-}+\Lambda, & \Lambda<\Lambda_{-} \\
\tilde{\zeta}_{0}(\Lambda), & \Lambda_{-} \leq \Lambda \leq \Lambda_{+} \\
\tilde{\zeta}_{0}\left(\Lambda_{+}\right)+\Lambda_{+}-\Lambda, & \Lambda>\Lambda_{+}\end{cases}
\end{aligned}
$$

where $\tilde{\zeta}_{0}^{\prime}\left(\Lambda_{ \pm}\right)=\mp 1$. If the FR holds for $\Lambda^{(0)}$, with $\left|\Lambda^{(0)}\right| \leq p^{*}\langle\Lambda\rangle$, the fact that $\tilde{\zeta}_{0}(\langle\Lambda\rangle)=0$ leads to

$$
\tilde{\zeta}(\Lambda)-\tilde{\zeta}(-\Lambda)= \begin{cases}\Lambda, & |\Lambda|<\langle\Lambda\rangle \\ \tilde{\zeta}_{0}(\Lambda)+\Lambda, & \langle\Lambda\rangle \leq|\Lambda| \leq \Lambda_{+} \\ \tilde{\zeta}_{0}\left(\Lambda_{+}\right)+\Lambda_{+}, & |\Lambda|>\Lambda_{+}\end{cases}
$$

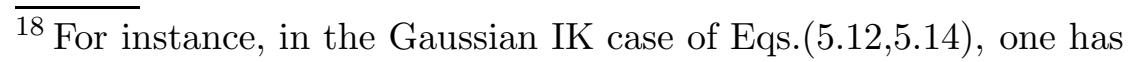

$$
\frac{d}{d t} V=\frac{1}{2 K} \sum_{i=1}^{N} \frac{\mathbf{p}_{\mathbf{i}}}{m} \cdot \mathbf{F}_{i}^{i n t}
$$


A relation similar to the heat FR of Van Zon and Cohen of [200] is thus obtained for $\Lambda$. The authors of [162] conclude that the $\Lambda^{(0)}$-FR holds with $|p| \leq p^{*}$, if $\Lambda^{(0)}$ is bounded or decays faster than exponential. They adopt Gentile's approach for Anosov flows, which reduces the flow to a Poincaré map [184], and assume that the Chaotic Hypothesis apply to a Poincaré map which avoids the singularities of $\Lambda$. This may be done taking a level section $V=\bar{V}$, for a certain value $\bar{V}$. Then, the volume contraction rate of the map, $\Lambda^{(0)}$, is bounded, the terms $\left(V_{f}-V_{i}\right)$ vanish, because $V_{i}=V_{f}=\bar{V}$, and the Chaotic Hypothesis yields the $\Lambda^{(0)}$-FR. Here, the connection with the Van Zon

- Cohen theory is made replacing the white noise with chaos due to uniform hyperbolicity.

\subsubsection{The Evans-Searles approach}

Because $\Lambda$ has no obvious physical meaning and the $\Lambda$-FR is based on strong assumptions which are not strictly enjoyed by systems of physical interest, one natural question comes to the fore: can one derive the steady state relation for the dissipated power divided by the kinetic energy, the $\Omega$-FR, under more physical assumptions? In [201], the Evans-Searles approach, first proposed in 1994 [202], has been polished to give an affirmative answer to this question.

Let us begin with the transient $\Omega$-FR. Let $\mathcal{M}$ be the phase space, $S^{\tau}: \mathcal{M} \rightarrow$ $\mathcal{M}$, a reversible evolution with time reversal involution operation $I$. Take a probability measure $d \mu(\Gamma)=f(\Gamma) d \Gamma$ on $\mathcal{M}$, and let the observable $\phi: \mathcal{M} \rightarrow$ $I R$ be odd with respect to time reversal (i.e. $\phi(I \Gamma)=-\phi(\Gamma)$ ). Introduce

$$
\bar{\phi}_{t_{0}, t_{0}+\tau}(\Gamma)=\frac{1}{\tau} \int_{t_{0}}^{t_{0}+\tau} \phi\left(S^{s} \Gamma\right) d s=\frac{1}{\tau} \phi_{t_{0}, t_{0}+\tau}(\Gamma)
$$

and the Dissipation Function

$$
\bar{\Omega}_{t_{0}, t_{0}+\tau}(\Gamma)=\frac{1}{\tau} \ln \frac{f\left(S^{t_{0}} \Gamma\right)}{f\left(S^{t_{0}+\tau} \Gamma\right)}+\bar{\Lambda}_{t_{0}, t_{0}+\tau}(\Gamma)
$$

for time even probability density $f$ (i.e. $f(I \Gamma)=f(\Gamma)$ ). The derivation that follows holds in full generality, but $\Omega$ equals the dissipated power only if $f$ is the equilibrium probability density for the given system, or is properly related to that. The existence of the logarithmic term of Eq.(5.37) has been called ergodic consistency [151]. For $\delta>0$, let $A_{\delta}^{+}=(A-\delta, A+\delta)$ and $A_{\delta}^{-}=(-A-\delta,-A+\delta)$, and let $E(\phi \in(a, b))$ be the set of points $\Gamma$ such that $\phi(\Gamma) \in(a, b)$. Then, $E\left(\bar{\Omega}_{0, \tau} \in A_{\delta}^{-}\right)=I S^{\tau} E\left(\bar{\Omega}_{0, \tau} \in A_{\delta}^{+}\right)$, and the transformation $\Gamma=I S^{\tau} X$ has Jacobian

$$
\left|\frac{d \Gamma}{d X}\right|=\exp \left(-\int_{0}^{\tau} \Lambda\left(S^{s} X\right) d s\right)=e^{-\Lambda_{0, \tau}(X)}
$$


which leads to

$$
\frac{\int_{E\left(\bar{\Omega}_{0, \tau} \in A_{\delta}^{+}\right)} f(\Gamma) d \Gamma}{\int_{E\left(\bar{\Omega}_{0, \tau} \in A_{\delta}^{-}\right)} f(X) d X}=\frac{\int_{E\left(\bar{\Omega}_{0, \tau} \in A_{\delta}^{+}\right)} f(\Gamma) d \Gamma}{\int_{E\left(\bar{\Omega}_{0, \tau} \in A_{\delta}^{+}\right)} \exp \left[-\Omega_{0, \tau}(X)\right] f(X) d X}
$$

i.e. to the transient $\Omega-F R$ :

$$
\frac{\mu\left(E\left(\bar{\Omega}_{0, \tau} \in A_{\delta}^{+}\right)\right)}{\mu\left(E\left(\bar{\Omega}_{0, \tau} \in A_{\delta}^{-}\right)\right)}=e^{[A+\epsilon(\delta, A, \tau)] \tau}, \quad|\epsilon| \leq \delta
$$

which concerns the non-invariant probability measure $\mu$ of density $f$. Time reversibility is essentially the only ingredient of the above derivation.

To obtain the steady state $\Omega-F R$, consider the ratio

$$
\frac{\mu\left(E\left(\bar{\phi}_{t_{0}, t_{0}+\tau} \in A_{\delta}^{+}\right)\right)}{\mu\left(E\left(\bar{\phi}_{t_{0}, t_{0}+\tau} \in A_{\delta}^{-}\right)\right)}=\frac{\int_{E\left(\bar{\phi}_{t_{0}, t_{0}+\tau} \in A_{\delta}^{+}\right)} f(\Gamma) d \Gamma}{\int_{E\left(\bar{\phi}_{t_{0}, t_{0}+\tau} \in A_{\delta}^{-}\right)} f(\Gamma) d \Gamma}
$$

and take $t=2 t_{0}+\tau$. Then

$$
E\left(\bar{\phi}_{t_{0}, t_{0}+\tau} \in A_{\delta}^{-}\right)=I S^{t} E\left(\bar{\phi}_{t_{0}, t_{0}+\tau} \in A_{\delta}^{+}\right)
$$

so that the transformation $\Gamma=I S^{t} W$, and the same algebra as above, yield

$$
\frac{\mu\left(E\left(\bar{\phi}_{t_{0}, t_{0}+\tau} \in A_{\delta}^{+}\right)\right)}{\mu\left(E\left(\bar{\phi}_{t_{0}, t_{0}+\tau} \in A_{\delta}^{-}\right)\right)}=\left\langle\exp \left(-\bar{\Omega}_{0, t_{0}} t_{0}\right)\right\rangle_{\bar{\phi}_{t_{0}, t_{0}+\tau} \in A_{\delta}^{+}}^{-1} .
$$

Move now the evolution from sets to measures, using

$$
\mu(E)=\mu_{t_{0}}\left(S^{t_{0}} E\right)=\int_{S^{t_{0}} E} f_{t_{0}}(W) d W
$$

where $E$ is a subset of $\mathcal{M}, \mu_{t_{0}}$ is the evolved measure up to time $t_{0}$, and $f_{t_{0}}$ its density. Some algebra yields

$$
\begin{aligned}
\frac{\mu_{t_{0}}\left(E\left(\bar{\phi}_{0, \tau} \in A_{\delta}^{+}\right)\right)}{\mu_{t_{0}}\left(E\left(\bar{\phi}_{0, \tau} \in A_{\delta}^{-}\right)\right)} & =\frac{\mu_{t_{0}}\left(S^{t_{0}} E\left(\bar{\phi}_{t_{0}, t_{0}+\tau} \in A_{\delta}^{+}\right)\right)}{\mu_{t_{0}}\left(S^{t_{0}} E\left(\bar{\phi}_{t_{0}, t_{0}+\tau} \in A_{\delta}^{-}\right)\right)} \\
& =\frac{\mu\left(E\left(\bar{\phi}_{t_{0}, t_{0}+\tau} \in A_{\delta}^{+}\right)\right)}{\mu\left(E\left(\bar{\phi}_{t_{0}, t_{0}+\tau} \in A_{\delta}^{-}\right)\right)}=\frac{1}{\left\langle\exp \left(-\bar{\Omega}_{0, t} t\right)\right\rangle_{\bar{\phi}_{t_{0}, t_{0}+\tau} \in A_{\delta}^{+}}}
\end{aligned}
$$

We call $\phi$-FR this relation. For $\bar{\phi}_{t_{0}, t_{0}+\tau}=\bar{\Omega}_{t_{0}, t_{0}+\tau}$, taking the logarithm and dividing by $\tau$, it produces:

$$
\begin{aligned}
& \frac{1}{\tau} \ln \frac{\mu_{t_{0}}\left(E\left(\bar{\Omega}_{0, \tau} \in A_{\delta}^{+}\right)\right)}{\mu_{t_{0}}\left(E\left(\bar{\Omega}_{0, \tau} \in A_{\delta}^{-}\right)\right)}= \\
& \quad A+\epsilon\left(\delta, t_{0}, A, \tau\right)-\frac{1}{\tau} \ln \left\langle e^{-t_{0}\left(\bar{\Omega}_{0, t_{0}}+\bar{\Omega}_{t_{0}+\tau, 2 t_{0}+\tau}\right)}\right\rangle_{\bar{\Omega}_{t_{0}, t_{0}+\tau} \in A_{\delta}^{+}}(5.4
\end{aligned}
$$


If $\mu_{t_{0}}$ tends to a steady state $\mu_{\infty}$ when $t_{0} \rightarrow \infty$, the above should change from a statement on the ensemble $f_{t_{0}}$, to a statement concerning also the statistics generated by a single typical trajectory. A result like this would be the steady state $\Omega$-FR. This requires further assumptions, because $t_{0}$ tends to infinity before $\tau$ does and, in principle, the growth of $t_{0}$ could make the conditional average in (5.47) diverge. Nevertheless, the decay of the auto-correlation of $\Omega$ suffices. Indeed, the conservation of probability yields

$$
\left\langle e^{-s \bar{\Omega}_{0, s}}\right\rangle=1, \quad \text { for every } s \in \mathbb{R}
$$

and if the $\Omega$-autocorrelation time vanishes, one can write:

$$
\begin{aligned}
\left\langle e^{-t_{0} \bar{\Omega}_{0, t_{0}}} e^{-t_{0} \bar{\Omega}_{t_{0}+\tau, 2 t_{0}+\tau}}\right\rangle_{\bar{\Omega}_{t_{0}, t_{0}+\tau} \in A_{\delta}^{+}}= & \\
\left\langle e^{-t_{0}\left(\bar{\Omega}_{0, t_{0}}+\bar{\Omega}_{t_{0}+\tau, 2 t_{0}+\tau}\right)}\right\rangle & =\left\langle e^{-t_{0} \bar{\Omega}_{0, t_{0}}}\right\rangle\left\langle e^{-t_{0} \bar{\Omega}_{t_{0}+\tau, 2 t_{0}+\tau}}\right\rangle
\end{aligned}
$$

and

$$
1=\left\langle e^{-s \bar{\Omega}_{0, s}-(t-s) \bar{\Omega}_{s, t}}\right\rangle=\left\langle e^{-\Omega_{0, s}}\right\rangle\left\langle e^{-\Omega_{s, t}}\right\rangle \Longrightarrow\left\langle e^{-(t-s) \bar{\Omega}_{s, t}}\right\rangle=1 \quad \text { for all } s, t .
$$

Hence, the logarithmic correction term in (5.47) identically vanishes for all $t_{0}, \tau$, and the $\Omega$-FR is verified even at short $\tau$ 's. Of course, this idealized situation does not need to be realized. Nevertheless, tests performed on molecular dynamics systems indicate that there exists a constant $K$, such that $[201,203]$

$$
0<\frac{1}{K} \leq\left\langle e^{-t_{0}\left(\bar{\Omega}_{0, t_{0}}+\bar{\Omega}_{t_{0}+\tau, 2 t_{0}+\tau}\right)}\right\rangle_{\bar{\Omega}_{t_{0}, t_{0}+\tau} \in A_{\delta}^{+}} \leq K
$$

As a matter of fact, the decorrelation (or Maxwell time), $t_{M}$, expresses a physical property of the system, thus it does not depend on $t_{0}$ or $\tau$, and depends only mildly on the external field [usually, $t_{M}\left(F_{e}\right)=t_{M}(0)+O\left(F_{e}^{2}\right)$ ]. Its order of magnitude is that of the mean free times. If these scenarios are realized, Eq.(5.51) follows and the logarithmic correction term of Eq.(5.47) vanishes as $1 / \tau$, with a characteristic scale of order $O\left(t_{M}\right)$.

It results that the steady state $\Omega$-FR can be obtained basically only from time reversibility and from the decay of the $\Omega$-autocorrelation, and one can write

$$
\frac{1}{\tau} \ln \frac{\mu_{\infty}\left(E\left(\bar{\Omega}_{0, \tau} \in A_{\delta}^{+}\right)\right)}{\mu_{\infty}\left(E\left(\bar{\Omega}_{0, \tau} \in A_{\delta}^{-}\right)\right)}=A+\text { small correction }
$$

where the small correction can be made arbitrarily small taking sufficiently large $\tau$ and sufficiently small $\delta$. This decay of correlations could be relaxed or replaced by other assumptions, but is needed for the convergence to a steady state, and is verified in most systems of physical interest. Furthermore, this approach explains why the relevant convergence times are functions of material 
properties of the systems, and do not diverge in the equilibrium limit. The decorrelation times of $\Omega$ are indeed material properties.

Various other relations can now be obtained. For instance, for any odd $\phi$, any $\delta>0$, any $t_{0}$ and any $\tau$, one has

$$
\left\langle\exp \left(-t \bar{\Omega}_{0, t}\right)\right\rangle_{\bar{\phi}_{t_{0}, t_{0}+\tau} \in(-\delta, \delta)}=1
$$

which, in the $\delta \rightarrow \infty$ limit, leads to the well known normalization property the so-called Nonequilibrium Partition Identity (5.48). Given a second density $\tilde{f}$ and the corresponding $\tilde{\Omega}$, one has

$$
\left\langle\exp \left(-t \bar{\Omega}_{0, t}\right)\right\rangle_{\bar{\phi}_{t_{0}, t_{0}+\tau} \in A_{\delta}^{+}} \approx \frac{\mu_{t_{0}}\left(E\left(\bar{\phi}_{0, \tau} \in A_{\delta}^{-}\right)\right)}{\mu_{t_{0}}\left(E\left(\bar{\phi}_{0, \tau} \in A_{\delta}^{+}\right)\right)} \approx\left\langle\exp \left(-t \bar{\Omega}_{0, t}\right)\right\rangle_{\bar{\phi}_{t_{0}, t_{0}+\tau} \in A_{\delta}^{+}}
$$

for large $t_{0}$, all $\tau$ and all allowed pairs $A$ and $-A$, if $d \mu=f d \Gamma$ and $\mathrm{d} \tilde{\mu}=\tilde{f} d \Gamma$ converge to the same steady state. Then, as far as the FRs are concerned, the different dissipation functions are equivalent. The Dissipation Relation

$$
\langle\phi\rangle_{t}=\int_{0}^{t} d s\langle\Omega(0) \phi(s)\rangle
$$

where $\langle.\rangle_{t}$ the average with respect to $\mu_{t}$, is another direct consequence of the approach followed in this section [204].

The theory outlined above is consistent with Irreversible Thermodynamics, since it leads to the Green-Kubo relations, close to equilibrium [161]; hence it can be considered in the construction of a more general theory, which encompasses far from equilibrium phenomena. Differently from Ref.[187], which deals with $\Lambda$ and with asymptotic times, Ref.[161] deals with $\Omega$ and stresses the role of the physical time scales. This is illustrated on a Nosé-Hoover thermostatted system [Eqs.(5.16)], whose equilibrium state is given by the extended canonical density

$$
f_{c}(\Gamma, \xi)=\frac{e^{-\beta\left(H_{0}+Q \xi^{2} / 2\right)}}{\int d \xi d \Gamma e^{-\beta\left(H_{0}+Q \xi^{2} / 2\right)}} \quad \text { with } \quad Q=2 K_{0} \theta=\frac{g \theta}{\beta}
$$

where $H_{0}$ is the internal energy, $K_{0}$ si the reference kinetic energy corresponding to the inverse temperature $\beta$, and $g$ depends on the number of degrees of freedom [17]. In the most common case, in which $\mathbf{F}_{i}^{\text {ext }}=c_{i} \mathbf{F}_{e}$ and $\mathbf{J}=\sum_{i=1}^{N} c_{i}\left(d \mathbf{q}_{i} / d t\right)$, one has

$$
\xi=-\frac{1}{2 K}\left[\frac{d H_{0}}{d t}+\mathbf{J} \cdot \mathbf{F}_{e} V\right]
$$

and

$$
\Lambda=-\operatorname{div}\left(\frac{d \Gamma}{d t}\right)-\partial_{\xi}\left(\frac{d \xi}{d t}\right)=d N \xi
$$


where $d$ is the spatial dimension, which shows that $\Lambda$ and its fluctuations are not directly related to the dissipation rate $\Omega=\beta \mathbf{J} \cdot \mathbf{F}_{e} V$, while its average is, because $\left\langle H_{0}\right\rangle=0$. Integrating $f_{c}(x, \xi)$ yields

$$
f_{c}(\xi)=\int d \Gamma f_{c}(\Gamma, \xi)=\sqrt{\frac{\beta Q}{2 \pi}} \exp \left[-\beta Q \xi^{2} / 2\right]
$$

Therefore, the distribution of $\bar{\xi}_{0, t}$ is also Gaussian in equilibrium, with variance proportional to that of $\bar{h}_{0, t}=(1 / t) \int_{0}^{t} \dot{H}_{0} d \tau$, and near equilibrium it can be assumed to remain Gaussian, around its mean, for large $t$, as a consequence of the Central Limit Theorem (CLT). To use the $\Lambda$-FR together with the CLT, the values $A$ and $-A$ of the random variable $\bar{\xi}_{0, t}$ must be a small number of standard deviations away from $\langle\Omega\rangle$. However, as explained in Refs. $[198,161,162]$ and briefly recalled in Section 5.4.1, the distribution of $d N \bar{\xi}_{0, t}$ verifies the $\Lambda$-FR only for times $t$ sufficiently large that the standard deviation of the random variable $\bar{h}_{0, t}$ is negligible with respect to that of $\bar{\Omega}_{0, t}$, $\sigma_{\bar{\Omega}_{0, t}}$ say.

In [156] it was proven that the variance of the average current obeys

$$
t \sigma_{\bar{J}_{0, t}}^{2}\left(F_{e}\right)=\frac{2 L\left(F_{e}\right) k_{B} T}{V}+O\left(\frac{F_{e}^{2}}{t N}\right)
$$

hence that of $\bar{\Omega}_{0, t}$ obeys

$$
t \sigma_{\Omega_{0, t}}^{2}\left(F_{e}\right)=\left(\frac{F_{e} V}{2 K_{0}}\right)^{2}\left[\frac{2 L\left(F_{e}\right) k_{B} T}{V}+O\left(\frac{F_{e}^{2}}{t N}\right)\right],
$$

where

$$
L\left(F_{e}\right)=\beta V \int_{0}^{\infty} d t\langle(J(t)-\langle J\rangle)(J(0)-\langle J\rangle)\rangle
$$

and $L(0)=\lim _{F_{e} \rightarrow 0} L\left(F_{e}\right)$ is the corresponding linear transport coefficient. Equation (5.58) shows that the standard deviation of the average current decreases when $t$ grows at fixed $F_{e}$, while it tends to a positive constant, when $F_{e}$ decreases at fixed $t$. Consequently, the standard deviation of $\bar{\Omega}_{0, t}$ tends to zero when $F_{e} \rightarrow 0$ at fixed $t$. Differently, the variance of $\bar{h}_{0, t}$ tends to a constant when $F_{e}$ tends to zero at fixed $t$. Therefore, the smaller $F_{e}$ the longer the time $t$ needed for $\sigma_{\bar{\Omega}_{0, t}}$ to dominate over the variance of $\bar{h}_{0, t}$, and this time grows without bounds when $F_{e}$ tends to zero. Let us assume for simplicity, that the variations of these standard deviations are monotonic when either $F_{e}$ or $t$ varies and the other variable is fixed. Then, given $F_{e}$ and $A$, there is $t_{\sigma}\left(F_{e}, A\right)$ such that the standard deviation of $\bar{\Lambda}_{0, t}$ is sufficiently large that $A$ and $-A$ are within a few standard deviations from the mean only if $t<t_{\sigma}\left(F_{e}, A\right)$. At the same time, let $t_{\delta}\left(F_{e}, A\right)$ be sufficiently large that the steady state $\Lambda$-FR applies to the values $A$ and $-A$, with accuracy $\delta$. To derive the Green-Kubo 
relations from the $\Lambda$-FR, one would need

$$
t_{\delta}\left(F_{e}, A\right)<t<t_{\sigma}\left(F_{e}, A\right)
$$

which is not satisfied if $t_{\delta}\left(F_{e}, A\right)$ grows too fast with decreasing $F_{e}$. This suggests that the derivation of the Green-Kubo relation from the $\Lambda$-FR is problematic, for systems like the Nosé-Hoover systems or the Gaussian isokinetic systems with singular interaction potentials. In Ref.[161] it is further shown that the validity of Eq.(5.60) is necessary but not sufficient to obtain the result.

Differently, there is no problem in obtaining the Green-Kubo relation from the $\Omega$-FR, because the corresponding convergence times do not diverge when $F_{e}$ tends to zero (as seen above, they become of the order of the Maxwell times), and because $\Omega$ is the observable of interest (the energy dissipation rate divided by the kinetic energy). For times $t$ of the order of the Maxwell time, one may then write [161]:

$$
\langle\Omega\rangle=\frac{t}{2} \sigma_{\bar{\Omega}_{0, t}}^{2} \quad \text { or } \quad L(0)=\lim _{F_{e} \rightarrow 0} \frac{\langle J\rangle}{F_{e}}=\beta V \int_{0}^{\infty} d s\langle J(0) J(s)\rangle
$$

where the first equality is due to the validity of the $\Omega$-FR and the second to $(5.59) 19$

\subsubsection{The Jarzynski and the Crooks relations}

A transient relation for the fluctuations of non-dissipative systems has been obtained by Jarzynski [163] 20 To introduce this relation, the author considers a Hamiltonian finite particles system, in equilibrium with a heat bath at temperature $T$, which is driven away from equilibrium, by performing some work $W$ on it. If $\lambda$ is a parameter that controls the Hamiltonian $H_{\lambda}$ say, the work is performed varying $\lambda$. Let $\lambda=\lambda(t)$ be a given protocol of duration $\tau$ for a variation of $\lambda$ from a value $\lambda(0)=A$ to another value $\lambda(\tau)=B$. Suppose that the same protocol is repeated many times, always in the same manner, and always from the equilibrium corresponding to $\lambda=A$, to build the statistics of the work done when $\lambda$ evolves from $A$ to $B$. Let $\rho$ be the PDF of the externally performed work and $\left\langle e^{-\beta W}\right\rangle_{A \rightarrow B}$ the average over all works done in varying $\lambda$ from $A$ to $B$. These works are not the thermodynamic work done on the system, if the process is not performed quasi-statically, in the presence of local equilibrium [205], but is nevertheless a measurable quantity.

$\overline{19}$ Equating Eqs.(33,34) in Ref.[161], one obtains $\beta V F_{e}=\langle J\rangle / t \sigma \bar{J}_{0, t}^{2}$. Then, the fact that $\Omega=\beta V F_{e} J$ implies $\beta V F_{e}=2\langle\Omega\rangle / t \sigma_{\bar{J}_{0, t}} \beta V F_{e}$, from which Eq.(5.61) follows.

20 The non-invariant ensemble, which characterizes the "transient" nature of the Jarzynski equality, is the initial canonical ensemble. 
The Jarzynski equality states that [163]:

$$
\left\langle e^{-\beta W}\right\rangle_{A \rightarrow B}=\int d W \rho(W) e^{-\beta W}=e^{-\beta[F(B)-F(A)]}
$$

where $\beta=1 / k_{B} T$, and $[F(B)-F(A)]$ is the free energy difference between the initial equilibrium, with $\lambda=A$ and the equilibrium state corresponding to $\lambda=B$. This state exists and is unique, hence the value of the state variable $F$ at $\lambda=B$ is well defined, but it does not need to be the actual state of the system at time $\tau$. Indeed, the work $W$ is performed on the system in the time interval $[0, \tau]$, while relaxation to the equilibrium corresponding to $\lambda=B$ may require longer times, depending on the explicit form of the protocol $\lambda(t)$. Note that the process always begins in the same equilibrium state corresponding to $\lambda=A$, but in different microscopic states. In general, at time $\tau$, the system will not even be in local equilibrium: Eq.(5.62) is supposed to hold for all possible protocols, hence even arbitrarily far from equilibrium (large $\frac{d}{d t} \lambda$ ). Therefore, the presence of the equilibrium quantities $F(A)$ and $F(B)$ may look puzzling. The puzzle is solved observing that the externally measured work does not need to coincide with the thermodynamic work (which would not differ from experiment to experiment, if performed quasi-statically, cf. Section 5.4.4). From an operational point of view, it does not matter whether the system is in local equilibrium or not: the externally applied forces can be measured, the resulting motions can be recorded, hence the externally performed works are defined and can be quantified. The difference of this process from a standard thermodynamic one is that the external work cannot be equated to the internal thermodynamic work, if the process is not performed quasi-statically.

The limitation of Jarzynski's derivation, that the dynamics be Hamiltonian, has been overcome by Evans in terms of NEMD models [206]. Therefore, the Jarzynsky equality, similarly to the transient $\Omega$-FR, results of quite wide applicability, since it does not rest on particular conditions on the microscopic dynamics. It is also consistent with the second law of thermodynamics, since it yields

$$
\langle\beta W\rangle_{A \rightarrow B} \geq \beta[F(B)-F(A)]
$$

because $\ln \langle\Phi\rangle \geq\langle\ln \Phi\rangle$ for positive observables $\Phi$, although $\langle W\rangle_{A \rightarrow B}$ is not a thermodynamic quantity in general.

Similarly, computing the ratio of the probability that the work done in the forward transformation is $W$, to the probability that it is $-W$ in the $B$ to $A$ transformation, with reversed protocol $-\frac{d}{d t} \lambda$, produces the Crooks relation [207]:

$$
\frac{\operatorname{Prob}_{A \rightarrow B}(W=a)}{\operatorname{Prob}_{B \rightarrow A}(W=-a)}=e^{-\beta[F(B)-F(A)]} e^{a}
$$

which leads to the Jarzynsky equality, by a simple integration: 


$$
\begin{aligned}
& \left\langle e^{-\beta W}\right\rangle_{A \rightarrow B}= \\
& \int \operatorname{Prob}_{A \rightarrow B}(W=a) e^{-a} d a=e^{-\beta[F(B)-F(A)]} \int \operatorname{Prob}_{B \rightarrow A}(W=-a) d a \\
& =e^{-\beta[F(B)-F(A)]} .
\end{aligned}
$$

A relation exists between these results and the $\Omega$-FR. In the first place, the transient $\Omega$-FR may be applied to the protocols of the Jarzynski equality and of the Crooks relation, [199]. Then, let $f_{A}$ and $f_{B}$ be the canonical distributions at same inverse temperature $\beta$, for the Hamiltonians $H_{A}$ and $H_{B}$. The corresponding Helmholtz free energies are $F_{i}=-k_{B} T \ln \int d \mathbf{q} d \mathbf{p} \exp \left[-H_{i} / k_{B} T\right]$ for $i=A, B$. For simplicity, let $\lambda$ go from $A$ to $B$ in a time $\tau$, with rate $\frac{d}{d t} \lambda=1 / \tau$, and from $B$ to $A$ with rate $\frac{d}{d t} \lambda=-1 / \tau$. Correspondingly, the microscopic thermostatted evolution equations are

$$
\begin{aligned}
& \frac{d}{d t} \mathbf{q}=\frac{\partial H_{\lambda}(\mathbf{q}, \mathbf{p})}{\partial \mathbf{p}}, \quad \frac{d}{d t} \mathbf{p}=-\frac{\partial H_{\lambda}(\mathbf{q}, \mathbf{p})}{\partial \mathbf{q}}-\epsilon_{i} \alpha(x) \mathbf{p}, \\
& \frac{d}{d t} \lambda= \pm \frac{1}{\tau}, \quad \text { with }+ \text { for } A \rightarrow B \text { and }- \text { for } B \rightarrow A
\end{aligned}
$$

where $\epsilon_{i}=1$ for $i=1, \ldots N_{w}$ and $\epsilon_{i}=0$ for $i>N_{w}$, so that the deterministic thermostat acts only on the $N_{w}$ particles of the walls of the system, to fix their kinetic temperature and mimic a heat bath at temperature $T$. Then, the work performed by the external forces is given by $\beta W=\beta\left[H_{B}-H_{A}\right]-\Lambda_{0, \tau}$.

If $A=B$, the $\Omega$-FR applies directly, and the Jarzynski equality is an immediate consequence of the $\Omega$-FR because the $\Omega$-FR implies

$$
\left\langle e^{-\beta W}\right\rangle=\int P(W) e^{-\beta W} d W=\int d W P(-W)=1 .
$$

The transient $\Omega$-FR, the Jarzynski equality and the Crooks relation do not have the same range of applicability, the Crooks relation being the most general. It is remarkable how all of them connect equilibrium to nonequilibrium properties of physical systems: the transient $\Omega$-FR deals with the energy dissipation associated with the application of a dissipative field to an ensemble of systems initially in equilibrium; the Jarzynski and Crooks relations relate the work done to the free energy differences. The point of view of NEMD is important in this context, because finite Hamiltonian systems do not afford nonequilibrium steady states and also because, in general, external drivings do not need to preserve the Hamiltonian nature of the dynamics. Therefore, the agreement, where appropriate, with the NEMD approach strengthens the results. The interest of all these relations is bound to grow with our understanding of microscopic systems, particularly in biophysics and nanotechnology. 
The connection between equilibrium and nonequilibrium properties made by the $\Omega$-FR, the Jarzynski equality and the Crooks relation is, in a sense, opposite to that made by the Fluctuation Response formula: while the relations treated in this subsection obtain information about equilibrium states from nonequilibrium dynamics, the Fluctuation Response formula uses equilibrium fluctuations to gain information on nonequilibrium states.

\subsubsection{Ensemble vs thermodynamic relations}

There is a substantial difference between the transient relations considered in this Section (see also, e.g. [208]), and the Fluctuation Response formula. The Fluctuation Response formula concerns thermodynamic properties of macroscopic objects, hence it holds for the behaviour of a single system. Differently, as previously observed, the transient relations concern the statistics of ensembles of objects. Nevertheless, the transient relations are sometimes confused for thermodynamic statements. This is due to the fact that the transient relations either have forms similar to the second law of thermodynamics, or can be used to obtain relations formally like the second law. Also, the statistical nature of the results concerning a collection of particle systems, is often confused for the statistics concerning the many particles of a single system. Obviously, this is not appropriate, and the importance of the transient relations must be found precisely in their unavoidable ensemble nature, not in their thermodynamic content. Indeed, the differences between the two kinds of relations have practical consequences, which can be understood as follows:

1. Thermodynamic statements do not refer to ensembles of systems, they concern single systems. If one has a collection of systems in the same thermodynamic state, they all behave in the same way, hence their average behaviour is, a fortiori, bound to be that of a single member of the collection. For this reason, one may resort to a microscopic statistical description, in which each single system is in a different microscopic state, in order to recover the unique common thermodynamic behaviour. This macroscopic behaviour emerges when the time and space scales are well separated from the microscopic scales, and sufficiently mixing processes take place. In that case, the differences among the ensemble members are not observable: they cannot be expressed in terms of thermodynamic quantities, such as the thermodynamic work $W$ done on a system. Equivalently, one may say that the differences vanish typically as $1 / \sqrt{N}$, where $N$ is the number of particles of each system in the collection. Thus, sufficiently large $N$ implies practically no differences in the behaviours of the collection members.

2. If work is done in a reversible fashion on a macroscopic system, one can draw the corresponding path of thermodynamic states in the, e.g. pressurevolume plane. Integrating along this path, one obtains a given value for the work done on the system, which is operationally accessible, by measuring 
the work done by the external equipment. In this case, the system and the working apparatus are globally in close contact all the time, and the energy given to the system, under a fixed protocol, is always the same. Therefore, the ensemble average and the single works are equal, and equal the internal and the external works.

3. If work is not done in a reversible fashion, one obtains different values for $W$, even if the same protocol is repeated. The energy that a single system may acquire from the experimental apparatus cannot be predicted, and only averages over many repetitions of the protocol may be inferred. The transient relations serve to this purpose. It is interesting to observe that this is the case even for large $N$. Indeed, given a large collection of systems, the different values of the works performed on them (not to be confused with fluctuations in the evolution of a single system) are not due to the size of $N$, but to the fact that the process is not quasi-static, and corresponds to no path in the space of thermodynamic states. The reason is that the energy exchanged between system and driving apparatus depends on the microscopic initial state, not just on the driving protocol. Every time the protocol is repeated, the microstate is different, hence a different value for $W$ is obtained. This value is unpredictable, because the initial microstate is unknown, moreover different initial ensembles lead to different statistics.

Therefore, the content of the ensemble relations in far from equilibrium processes is not, e.g. the impossible quantification of the dissipative works, but the statistical description of large collections of systems. Indeed, they describe intriguing properties of the chosen initial ensembles. The fact that only in certain limits are the transient relations equivalent to thermodynamic statements is not a difficulty, because they have the advantage of being valid even when the thermodynamic description fails (e.g. in the absence of local equilibrium, or for systems of a small number of particles).

\section{Fluctuation relations in stochastic systems and applications}

\subsection{Stochastic systems: bounded and unbounded state spaces}

The derivations of the FR for stochastic systems are much easier than those of the $\Lambda$-FR, because, as noted in [209], the difficulties connected with singular invariant phase space distributions are avoided, and one can deal with smooth probability distributions. 21 The first of the stochastic FRs was pro-

$\overline{{ }^{21} \text { Note }}$ that for the same reason the derivation of the $\Omega$-FR given in Section 5 , which deals with the evolution of probability densities, is technically much easier, and of wider applicability than that of the $\Lambda$-FR. 
duced by Kurchan, who obtained a modified detailed balance property for Langevin processes of finite systems, and a FR for the entropy production, under a few assumptions, like e.g. the boundedness of the potential [164]. Lebowitz and Spohn [210] extended this result to generic Markov processes with a finite number of states. Additionally, under the assumption that local detailed balance is attained, they showed that the Gibbs entropy production is related to the action functional that satisfies the FR. This means that for Markov processes the Gibbs entropy production plays the role of the phase space contraction rate in the $\Lambda$-FR. Maes obtained a large deviation principle for discrete space-time Gibbs measures [211]. He showed that using the Gibbs formalism to describe the steady state of time discrete lattice systems leads to a FR for a kind of Gibbs entropy production. These results can be seen as a generalization of the $\Lambda$-FR and of its stochastic versions, as stochastic dynamics and thermostatted systems satisfying the chaotic hypothesis are particular cases of space-time Gibbs measures.

Farago pointed out that singularities may cause difficulties in the conventional use of the FR, for stochastic systems [212]. Wang, et al., reported the experiment of a colloidal particle that is dragged through water by means a moving optical trap [213], confirming an integrated version of the transient FR. This experiment may be modeled through a stochastic Langevin evolution describing a Brownian particle, dragged in a liquid by a moving harmonic potential, a problem studied by various authors $[212,214,215,216]$. In particular, analyzing the results of [213], Van Zon and Cohen [200] observed that, in the presence of a potential term, the work done on the system in a time $\tau, W_{\tau}$, consists of two parts: the heat $Q_{\tau}$ corresponding to the energy dissipated in the liquid, and the potential energy difference of the Brownian particle $\Delta U_{\tau}$ :

$$
W_{\tau}=Q_{\tau}+\Delta U_{\tau}
$$

A Brownian particle in a fluid, subjected to a harmonic potential that moves with a constant velocity $v^{*}$, is described by the overdamped Langevin process

$$
\frac{d x(t)}{d t}=-\left[x(t)-x^{*}(t)\right]+\zeta(t)
$$

where $x(t)$ is the position of the particle at time $t, x^{*}(t)=v^{*} t$ is the prescribed position of the minimum of the potential and $\zeta(t)$ is a white noise term representing the fluctuating force the fluid exerts on the particle. The units used are such that $k_{B} T=1$. The total work is given by

$$
W_{\tau}=-v^{*} \int_{0}^{\tau}\left[x(t)-x^{*}(t)\right] d t .
$$

In a comoving frame Eq. (6.2) reduces to a standard Ornstein-Uhlenbeck process and thus, the stationary probability distribution and Green's function are Gaussian in the particle's position. Since the total work is linear in the 
particle's position, $W_{\tau}$ is Gaussian as well. Because of this and of Eq. (6.3), in the case of transient fluctuations, the variance of $W_{\tau}$ equals $2\left\langle W_{\tau}\right\rangle$. Since $W_{\tau}$ is Gaussian, the total work satisfies the transient FR. In the $\tau \rightarrow \infty$ limit, the variance of $W_{\tau}$ remains twice its mean, hence the total work satisfies the steady state FR.

Van Zon and Cohen pointed out that the quantity measured in the experiment of [213] was precisely the total work and thus, in agreement with their Langevin treatment. However, they also clarified that, while the PDF of the total work is Gaussian, at equilibrium the PDF of the potential energy is exponential: $P(\Delta U) \sim \exp (-$ const. $\Delta U)$, and is expected to remain such in the steady states. Therefore, while the small fluctuations of heat are expected to coincide with those of the total work, since the contribution of the potential energy is only $\mathcal{O}(1)$, large heat fluctuations are more likely to be due to a large fluctuation of the potential energy.

Consider the harmonic potential $U(t) \equiv \frac{1}{2}\left|x(t)-x^{*}(t)\right|^{2}$ in Eq. (6.1). The heat $Q_{\tau}$ is nonlinear in the particle's position, hence its PDF needs not be Gaussian. Compute the Fourier transform of this PDF:

$$
\hat{P}_{\tau}(q) \equiv \int_{-\infty}^{\infty} d Q_{\tau} e^{i q Q_{\tau}} \operatorname{Prob}_{\tau}\left(Q_{\tau}\right)
$$

Writing $\operatorname{Prob}_{\tau}\left(Q_{\tau}\right)$ in terms of the joint distribution of the work $W_{\tau}$ and of the positions $x(0), x(\tau)$, one obtains

$$
\hat{P}_{\tau}(q)=\frac{\exp \left\{w(i-q)\left(\tau-\frac{2 q^{2}\left(1-e^{-\tau}\right)^{2}}{1+\left(1-e^{-2 \tau}\right) q^{2}}\right)\right\}}{\left[1+\left(1-e^{-2 \tau}\right) q^{2}\right]^{3 / 2}}
$$

where $w=\left\langle W_{\tau}\right\rangle / \tau$ is the rate of work done in the system, related to the stationary state average. Anti-transforming $\hat{P}_{\tau}(q)$, one considers the heat fluctuation function

$$
f_{\tau}(p)=\frac{1}{w \tau} \ln \left[\frac{\operatorname{Prob}_{\tau}(p w \tau)}{\operatorname{Prob}_{\tau}(-p w \tau)}\right],
$$

where $p=Q_{\tau} /\left\langle Q_{\tau}\right\rangle$ and $\left\langle Q_{\tau}\right\rangle=\left\langle W_{\tau}\right\rangle-\left\langle\Delta U_{\tau}\right\rangle=w \tau$, since $\left\langle\Delta U_{\tau}\right\rangle=0$, in the steady state. To obtain an asymptotic analytical expression of Eq. (6.6), consider the quantity

$$
e(\lambda) \equiv \lim _{\tau \rightarrow \infty}-\frac{1}{w \tau}\left\langle e^{-\lambda Q_{\tau}}\right\rangle
$$

for large $\tau$, and

$$
\operatorname{Prob}_{\tau}\left(Q_{\tau}\right) \sim e^{-w \tau \hat{e}\left(Q_{\tau} / w \tau\right)}
$$

where $\hat{e}(p)=\max _{\{\lambda\}}[e(\lambda)-\lambda p]$ is the Legendre transform of $e(\lambda)$. Analytically 
continuing $\hat{P}_{\tau}$ to imaginary arguments, one can write $\left\langle e^{-\lambda Q_{\tau}}\right\rangle=\hat{P}_{\tau}(i \lambda)$, i.e.

$$
\left\langle e^{-\lambda Q_{\tau}}\right\rangle=\frac{\exp \left[-w \lambda(1-\lambda)\left\{\tau+\frac{2 \lambda^{2}\left(1-e^{-\tau}\right)^{2}}{1-\left(1-e^{-2 \tau}\right) \lambda^{2}}\right\}\right]}{\left[1-\left(1-e^{-2 \tau}\right) \lambda^{2}\right]^{3 / 2}}
$$

which is singular for $\lambda= \pm\left(1-e^{-2 \tau}\right)^{-1 / 2}$. Using Eqs. (6.7) and (6.9) and taking the $\tau \rightarrow \infty$ limit, the singularities move to \pm 1 , yielding

$$
e(\lambda)=\lambda(1-\lambda) \quad \text { for }|\lambda|<1
$$

This implies that the conventional steady state $\mathrm{FR}, \lim _{\tau \rightarrow \infty} f_{\tau}(p)=p$, holds as in Ref.[210]. Outside the interval $(-1,1)$, the integral in Eq. (6.8) diverges due to the exponential tails of the PDF of $Q_{\tau}: e(\lambda)=-\infty$ for $|\lambda|>1$. Finally, taking these values in the Legendre transform $\hat{e}(p)$, and substituting in Eq. (6.8) one obtains

$$
\lim _{\tau \rightarrow \infty} f_{\tau}(p)=\left\{\begin{array}{lll}
p & \text { for } & 0 \leq p<1 \\
p-(p-1)^{2} / 4 & \text { for } & 1 \leq p<3 \\
2 & \text { for } & p \geq 3
\end{array}\right.
$$

and the values for negative $p$ are obtained from $f_{\tau}(-p)=-f_{\tau}(p)$. The fluctuations of heat that are smaller than $\left\langle Q_{\tau}\right\rangle$ satisfy the conventional $\mathrm{FR}$, as those of $W_{\tau}$; larger heat fluctuations behave differently, in particular, the probability ratio $f_{\tau}$ is much larger than that predicted by the conventional FR. A similar phenomenon can occur in deterministic systems [161,162], and the logarithmic term in the definition of $\Omega$, Eq.(5.37), represents the corresponding boundary terms.

The validity of the extended FR of Van Zon and Cohen has been addressed in other stochastic systems. For instance, Ref.[217], where a granular system is also considered, studies the extended FR, and shows that the boundary terms may be important in many common situations. One interesting feature of the models considered in [217], is that the granular system behaves very similarly to a simple Markov chain with unbounded state space, as discussed in Subsection 5.4.1. Somewhat different results are reported in Ref.[218], which shows that the extended FR does not hold in the partially asymmetric zerorange process with open boundaries. Hence the range of validity of the Van Zon - Cohen scenario is still to be fully understood.

Various other studies have dealt with the problem of a Brownian particle and Langevin process in general, like those of Refs.[219,220,221,222,209,223,224,225]. In particular, Ref.[223] points out that exponential distributions of the boundary terms may not always have the same effect on FRs, hence the problem of their relevance in FR remains open. 


\begin{tabular}{|c|c|c|c|}
\hline Relation & State & Dynamics & Observable \\
\hline$(5.1)$ & NESS & Gaussian thermostatted & $\mathrm{DP}$ \\
\hline$(5.22)$ & NESS & Reversible transitive Anosov & $\Lambda$ \\
\hline$(5.29)$ & NESS & Reversible transitive Anosov & Local- $\Lambda$ \\
\hline$(5.35)$ & NESS & $\begin{array}{c}\text { Reversible flow with transitive } \\
\text { Anosov Poincaré map }\end{array}$ & Local- $\Lambda$ \\
\hline$(5.39)$ & SMe-NE & Reversible and dissipative & $\Omega$ \\
\hline$(5.43)$ & SMe-NE & Reversible and dissipative & $\phi$ \\
\hline$(5.48)$ & SMe-NE & Reversible and dissipative & $\Omega$ \\
\hline$(5.55)$ & SMe-NE & Reversible and dissipative & $\Omega$ \\
\hline$(5.52)$ & NESS & $\begin{array}{l}\text { Reversible and dissipative } \\
\Omega \text {-correlation decay with } \\
\text { respect to initial measure }\end{array}$ & $\Omega$ \\
\hline$(5.62)$ & $\mathrm{C}-\mathrm{NE}$ & Hamiltonian dynamics & $F$ \\
\hline$(5.64)$ & $\mathrm{C}-\mathrm{NE}$ & $\begin{array}{l}\text { Hamiltonian dynamics } \\
\text { or Markov process }\end{array}$ & $F$ \\
\hline$(6.11)$ & NESS & $\begin{array}{l}\text { Markovian model of } \\
\text { colloidal particle in optical trap }\end{array}$ & $H F$ \\
\hline
\end{tabular}

Table 1. Synoptic table of fluctuation relations. The first column gives the equation number. The second column gives the details of the state for which the relation holds: NESS means nonequilibrium steady state, C-NE means experiments starting in canonical equilibrium and continuing under nonequilibrium dynamics, SMe-NE means initial smooth ensemble followed by nonequilibrium dynamics. The third column specifies the dynamics for which the relation has been obtained. In the fourth column, the observables for which the relation holds are given: DP means dissipated power, $\Lambda$ means phase space contraction rate, $\Omega$ means dissipation function, $\phi$ is any odd observable, $F$ is the Helmholtz free energy. Local means integrated over a given region of the relevant space, HF means heat flux.

\subsubsection{Markov chains with an infinite number of states}

The problem of singularities in unbounded spaces can be understood in detail using a simple model [226,217]. Consider a Markov chain with $N$ (possibly 
infinite) states with invariant probability measure $\boldsymbol{\mu}=\left\{\mu_{\sigma}\right\}$ and transition rates $K_{a b}$ which denote the conditional probability of going from state $a$ to state $b$. We are interested in the fluctuations of the following two "action functionals":

$$
\begin{aligned}
W(\tau) & =\sum_{i=0}^{\tau} \log \frac{K_{\sigma_{i} \sigma_{i+1}}}{K_{\sigma_{i+1} \sigma_{i}}} \\
W^{\prime}(\tau) & =\sum_{i=0}^{\tau} \log \frac{K_{\sigma_{i} \sigma_{i+1}}}{K_{\sigma_{i+1} \sigma_{i}}}+\log \frac{\mu_{\sigma_{0}}}{\mu_{\sigma_{\tau+1}}}
\end{aligned}
$$

where $\sigma_{i} \in\{1,2, \ldots N\}$ is the state of the system at time $i$. Both functionals have been defined by Lebowitz and Spohn, in [227], who focused on the properties of $W$, neglecting the importance of the difference $B=W^{\prime}-W$. Both $W$ and $W^{\prime}$ associate a real number to any finite trajectory (any realization of the Markov chain). In a stationary state which verifies the detailed balance condition $\mu_{a} K_{a b}=\mu_{b} K_{b a}$, one has $W^{\prime}(t) \equiv 0$, while $W(t) \neq 0$ (except when $K_{a b}=K_{b a}$ ). For $t$ large enough, almost all the trajectories yield $\lim _{s \rightarrow \infty} W(s) / s=\lim _{s \rightarrow \infty} W^{\prime}(s) / s=\langle W(t) / t\rangle=\left\langle W^{\prime}(t) / t\right\rangle$, where (assuming an ergodic and stationary system) \langle\rangle indicates an average over many independent segments from a single very long trajectory. Let $S=-\sum_{i=1}^{N} \nu_{i} \log \nu_{i}$ be the Gibbs entropy of the system at time $t$, where $\nu_{i}(t)$ is the probability to be in the state $i$ at time $t$; then

$$
S(t+1)-S(t)=\Sigma(t)-A(t)
$$

where $\Sigma(t)$ is always non-negative, $A(t)$ is a linear function with respect to $\boldsymbol{\nu}(t)=\left\{\nu_{i}(t)\right\}$, and $\langle W(t)\rangle=\left\langle W^{\prime}(t)\right\rangle \equiv \int_{0}^{t} \mathrm{~d} t^{\prime} A\left(t^{\prime}\right)$. In [227] this has been shown for continuous time Markov processes, but the proof is valid also in the discrete time case (see for example [228]). This leads to consider $W(t)$ and $W^{\prime}(t)$ equivalent for the contribution of a single trajectory to the total entropy flux. In a stationary state $A(t)=\Sigma(t)$ and therefore the flux is equivalent to the production. For large enough $t$ one has:

(1) at equilibrium (i.e. when there is detailed balance) $\langle W(t)\rangle=\left\langle W^{\prime}(t)\right\rangle=0$;

(2) out of equilibrium $\langle W(t)\rangle>0$ and $\left\langle W^{\prime}(t)\right\rangle>0$.

Consider the fluctuations of the functional $W$. We first define an extended probability vector $\mathbf{p}(t, W)$, whose component $p_{i}(t, W)$ is the probability of finding the system at time $t$ in the state $i$, with value $W$ for the Lebowitz and Spohn functional. This means that $\sum_{i} p_{i}(t, W)=f(t, W)$ is the probability density function at time $t$ for the functional $W$, while $\int \mathrm{d} W p_{i}(t, W)=\nu_{i}(t)$ is the probability of finding the system in state $i$ at time $\mathrm{t}$ and $\int \mathrm{d} W \sum_{i} p_{i}(t, W)=$ 
1. The evolution of $\mathbf{p}(t, W)$ is given by the following equation:

$$
p_{i}(t+1, W)=\sum_{j} K_{j i} p_{j}\left(t, W-\Delta w_{j i}\right)
$$

where $K$ is the previously defined transition matrix and $\Delta w_{i j}$ is the variation of $W$ due to a jump from the state $i$ to the state $j$. This reads $\Delta w_{i j}=\ln \frac{K_{i j}}{K_{j i}}$. Then define the function $\tilde{\mathbf{p}}(t, \lambda)=\int \mathrm{d} W \exp (-\lambda W) \mathbf{p}(t, W)$ and obtain, for its evolution

$$
\tilde{\mathbf{p}}(t+1, \lambda)=A(\lambda) \tilde{\mathbf{p}}(t, \lambda)
$$

$A(\lambda)$ being the matrix defined by

$$
A_{i j}(\lambda)=K_{j i}^{1-\lambda} K_{i j}^{\lambda}
$$

and, therefore,

$$
\tilde{\mathbf{p}}(t, \lambda)=A(\lambda)^{t} \tilde{\mathbf{p}}(0, \lambda)
$$

with $\tilde{p}_{i}(0, \lambda)=\int \mathrm{d} W \exp (-\lambda W) \nu_{i}(0) \delta(W)=\nu_{i}(0)$.

The characteristic function of the distribution of $W$ is obtained summing over all the states:

$$
\tilde{f}(t, \lambda)=\sum_{i} \tilde{p}_{i}(t, \lambda)=\sum_{i} \sum_{j}\left[A(\lambda)^{t}\right]_{i j} \nu_{j}(0)=\sum_{j} \nu_{j}(0) \sum_{i}\left[A(\lambda)^{t}\right]_{i j}
$$

The distribution of $W$ at time $t$ is recovered by inverting the Laplace transform:

$$
f(t, W)=\int_{-i \infty}^{+i \infty} \mathrm{d} \lambda \exp (\lambda W) \tilde{f}(t, \lambda)
$$

Furthermore, the knowledge of the characteristic function suffices to compute the moments or the cumulants of $W$ :

$$
\begin{aligned}
\left\langle W_{t}^{n}\right\rangle & =\left.(-1)^{n} \frac{d^{n}}{d \lambda^{n}} \tilde{f}(t, \lambda)\right|_{\lambda=0} \\
\left\langle W_{t}^{n}\right\rangle_{c} & =\left.(-1)^{n} \frac{d^{n}}{d \lambda^{n}} \log \tilde{f}(t, \lambda)\right|_{\lambda=0} .
\end{aligned}
$$

At large times, the evolution operator $A(\lambda)^{t}$ is dominated by the largest eigenvalue $y_{1}(\lambda)$ of $A(\lambda)$. Defining $y_{1}(\lambda)=\exp (\zeta(\lambda))$ it follows that

$$
\begin{aligned}
\tilde{\mathbf{p}}(t, \lambda) & \sim \exp (\zeta(\lambda) t)\left[\sum_{j} x_{j}^{(1)}(\lambda) \nu_{j}(0)\right] \mathbf{x}^{(1)}(\lambda) \\
\tilde{f}(t, \lambda)=\sum_{i} \tilde{p}_{i}(t, \lambda) & \sim \exp (\zeta(\lambda) t)\left[\sum_{j} x_{j}^{(1)}(\lambda) \nu_{j}(0)\right] \sum_{i} x_{i}^{(1)}(\lambda),
\end{aligned}
$$


where $\mathbf{x}^{(1)}(\lambda)$ is the eigenvector of $A(\lambda)$ associated to the largest eigenvalue $y_{1}(\lambda)$. One expects, from the above large times behavior, an analogous large time behavior for the density distribution of $W$, i.e.

$$
\begin{aligned}
f(t, W)=\int_{-i \infty}^{+i \infty} \mathrm{d} \lambda \exp (\lambda W) \sum_{j} \nu_{j}(0) \sum_{i}\left[A(\lambda)^{t}\right]_{i j} \\
\sim \exp \left[t \max _{\lambda}\left(\lambda \frac{W}{t}+\zeta(\lambda)\right)\right]=\exp [t \pi(W / t)]
\end{aligned}
$$

where we have introduced $\pi(w)=\lim _{t \rightarrow \infty} \frac{1}{t} \log f(t, w t)$, the large deviation function associated with $f(t, W)$, which is obtained as a Legendre transform of $\zeta(\lambda)$, i.e. $\pi(w)=\lambda^{*} w+\zeta\left(\lambda^{*}\right)$ with $\left.\frac{d}{d \lambda} \zeta(\lambda)\right|_{\lambda=\lambda^{*}}=-w$, under the validity of the last chain of equalities.

The previously defined Fluctuation Relation appears at this stage. In fact, it is evident that $A(\lambda)=A^{T}(1-\lambda)$, being $A^{T}$ the transpose of $A$. This implies that $\zeta(\lambda)=\zeta(1-\lambda)$ which suffices to get $\pi(w)=\pi(-w)+w$, i.e. $f(t, W)=f(t,-W) \exp (W)$ at large times. The validity of the expansion in (6.22) is crucial for the validity of this relation, but it is not guaranteed when the integrand presents non-analyticities in the $\lambda$ complex plane. Such a catastrophe can happen, for example, when the number of states $N$ becomes infinite and the initial (final) probability $\mu_{j(i)}(0)$ has some unbounded form (see below). The physical meaning of such a catastrophe is that the large fluctuations in the initial and final state cannot be neglected, because they contribute to the tails (i.e. the large deviations) of $f(t, W)$ at all times. Note that the breaking of analyticity of $\tilde{f}(t, \lambda)$ is associated with large fluctuations of invariant probabilities. Many studies, including numerical simulations [226], analytical calculations [229,230,223] and heuristic arguments [231], show that the initial and final configurations modify the standard picture when the distribution of the boundary term $B=\ln \mu_{\sigma(0)}-\ln \mu_{\sigma(t)}$ has exponential, or higher, tails in the stationary state. This may occur even if $\mu_{i}$ has Gaussian tails.

Let us see what happens to the functional $W^{\prime}$ which differs from $W$ only for the non time-extensive term $B$, letting the prime denote quantities which involve this functional. For example, we denote the extended measure vector by $\mathbf{p}^{\prime}\left(t, W^{\prime}\right)$ and the probability density function at time $t$ by $f^{\prime}\left(t, W^{\prime}\right)$, the characteristic function by $\tilde{f}^{\prime}(t, \lambda)$, etc. The increment of the functional at each jump reads $\Delta w_{i j}^{\prime}=\Delta w_{i j} \log \left(\frac{\mu_{i}}{\mu_{j}}\right)$, recalling that $\mu_{i}$ is the invariant measure for the state $i$. The evolution matrix therefore reads

$$
A_{i j}^{\prime}(\lambda)=K_{j i}^{1-\lambda} K_{i j}^{\lambda} \mu_{j}^{-\lambda} \mu_{i}^{\lambda},
$$

and the evolution equation reads

$$
\tilde{\mathbf{p}}^{\prime}(t+1, \lambda)=A^{\prime}(\lambda) \tilde{\mathbf{p}}^{\prime}(t, \lambda) .
$$


The fundamental difference between $W^{\prime}$ and the original $W$ appears now. Since $\tilde{\mathbf{p}}^{\prime}(0, \lambda)=\boldsymbol{\nu}(0)$, if one takes $\boldsymbol{\nu}(0) \equiv \boldsymbol{\mu}$ (the system is in the stationary regime from the beginning), it happens that

$$
\tilde{f}^{\prime}(1, \lambda)=\sum_{i} \tilde{p}_{i}^{\prime}(1, \lambda)=\sum_{i} \sum_{j} A_{i j}^{\prime}(\lambda) \mu_{j}=\sum_{i} \sum_{j} K_{j i}^{1-\lambda} K_{i j}^{\lambda} \mu_{j}^{1-\lambda} \mu_{i}^{\lambda}
$$

i.e. $\tilde{f}^{\prime}(1, \lambda)=\tilde{f}^{\prime}(1,1-\lambda)$. By recursion, one realizes that this is the case for all times $t$, i.e.

$$
\tilde{f}^{\prime}(t, \lambda)=\tilde{f}^{\prime}(t, 1-\lambda)
$$

in general, which leads immediately to a finite-time symmetry relation

$$
f^{\prime}\left(t, W^{\prime}\right)=f^{\prime}\left(t,-W^{\prime}\right) \exp \left(W^{\prime}\right)
$$

valid for any $t$.

The functional $W^{\prime}$ contains a term that cancels the effects of the fluctuations of the steady state measure, leading to a conservation of the symmetry $\lambda \rightarrow 1-\lambda$ all along the evolution. Such a conservation prevents surprises at large times also in presence of large fluctuations of the initial measure.

As an example of the above discussion [217], consider a Markov chain with $N+2$ states, labeled $A, B$ and $C_{i}$ with $i \in\{1,2, \ldots N\}$ and with a transition matrix $K$ defined by

$$
\left(\begin{array}{cccccc}
p_{A A} & p_{A B} & \left(1-p_{A A}-p_{A B}\right) k_{1} & \left(1-p_{A A}-p_{A B}\right) k_{2} & \cdots & \left(1-p_{A A}-p_{A B}\right) k_{N} \\
p_{B A} & 0 & \left(1-p_{B A}\right) k_{1} & \left(1-p_{B A}\right) k_{2} & \cdots & \left(1-p_{B A}\right) k_{N} \\
p_{C A} 1-p_{C A} & 0 & 0 & \cdots & 0 \\
p_{C A} & 1-p_{C A} & 0 & 0 & \cdots & 0 \\
\vdots & \vdots & & & &
\end{array}\right)
$$

with $\sum_{i=1}^{N} k_{i}=1$, under the constraint that, for every jump of positive transition probability, the reversed jump is also possible. The invariant probability $\boldsymbol{\mu}$ for $N=1$ is given by

$$
\begin{aligned}
\mu_{A} & =\frac{p_{B A}+p_{C A}-p_{B A} p_{C A}}{\mathcal{N}} \\
\mu_{B} & =\frac{1-p_{A A}+p_{C A}\left(p_{A B}+p_{A A}-1\right)}{\mathcal{N}} \\
\mu_{C} & =\frac{1-p_{A A}-p_{A B} p_{B A}}{\mathcal{N}}
\end{aligned}
$$

with $\mathcal{N}=2+p_{A A}\left(-2+p_{C A}\right)+p_{A B} p_{C A}-p_{B A}\left(-1+p_{A B}+p_{C A}\right)$. 
It can be easily seen that the invariant measure for $N>1$ is closely related to the case $N=1$ with a decomposition of the measure of state $C_{1}$ into the measures of $C_{i}$ proportional to the values $k_{i}$ :

$$
\mu_{C_{i}}=\mu_{C} k_{i}
$$

In numerical simulations of this model [217], the functionals $W(\tau)$ and $W^{\prime}(\tau)$ can be measured along independent non-overlapping segments of duration $\tau$, extracted from a unique trajectory after the stationary regime has been achieved. Then, the following relation can be verified:

$$
G_{\tau}(X)=\log F(\tau, X)-\log F(\tau,-X)=X
$$

where $F(t, X)$ is the probability density function of finding one of the two functionals $W$ or $W^{\prime}$ after a time $t$ equal to $X$ (i.e. $F(t, X)$ corresponds to $f(t, W)$ or to $f^{\prime}\left(t, W^{\prime}\right)$ depending on the cases). Some typical results are shown in figures 16, which show the graph of $G_{\tau}$ vs $x$ and the pdfs of $W$ and $W^{\prime}$. The phenomenology can be divided in 3 main cases, depending on the choice of the transition rates. Whenever detailed balance is satisfied (here when $A$ is disconnected from all $C_{i}$ 's, i.e. when $p_{A B}=1-p_{A A}$ and $\left.p_{C A}=0\right), W^{\prime}(\tau)$ is identically zero and does not fluctuate (its pdf is a delta in zero), as expected, and therefore $W(\tau)$ coincides with the opposite of the boundary term $B=\log \frac{\mu_{\sigma_{1}}}{\mu_{\sigma_{\tau}}}$ : they both have symmetric fluctuations around zero with exponential tails, and $G_{\tau} \equiv 0$. When detailed balance is violated (i.e. when $A$ is connected to all $C_{i}$ 's) both $W(\tau)$ and $W^{\prime}(\tau)$ fluctuate around a nonzero (positive) value which, for $\tau$ large enough, is the same for the two functions. In general the fluctuations of the boundary term $B=\log \frac{\mu_{\sigma_{1}}}{\mu_{\sigma_{\tau}}}$ have a pdf with exponential tails and the pdf of $W^{\prime}$ is almost perfectly Gaussian. This is not true for the pdf of $W$. At small times $(\tau=100)$ the pdf of $W$ resembles the pdf of $B$, evidencing that at this time $W$ is completely dominated by $B$. At larger times the pdf of $W$ and $B$ start to deviate, in particular that of $W$ tends to that of $W^{\prime}$ in the bulk, with evidently different tails of exponential form. The tails of the pdf of $W$ are always dominated by the fluctuations of $B$. The symmetry relation for the functional $W$ is not verified in any of these simulations, both at small and large times. The asymmetry $G_{\tau}(W)$ has a slope near 1 only for small values of $W \ll\langle W\rangle$, then deviates and saturates to a constant value in good agreement with the value $2\langle W\rangle$ predicted by van Zon and Cohen [231]. Note that the observations at large times are perfectly compatible with those at small times, i.e. if both ordinates and abscissas are divided by $\tau$, the curves are similar (in the first two cases they overlap very well). Therefore the "reduction" of the violation at large times is only apparent. On the other hand the symmetry relation for $W^{\prime}$ is always satisfied, at all times and for all the choices of the parameters. Finally, when the choice of the rates $k_{i}$ is such that the invariant measure on states $C_{i}$ is an exponential with a very high slope, the numerical results show that the fluctuations of $W$ are much closer 
to those of $W^{\prime}$ already at small times. This is reflected on the good agreement with the FR of both functionals $W$ and $W^{\prime}$. One can still be doubtful about this verification, because of the limited range of values of $W$ available, which can possibly hide a failure at larger values. The distribution of the boundary term, in fact, still has exponential tails (invisible at our resolution) and it can be argued that these tails (being Gaussian those of $W$ ) will dominate at very large values.
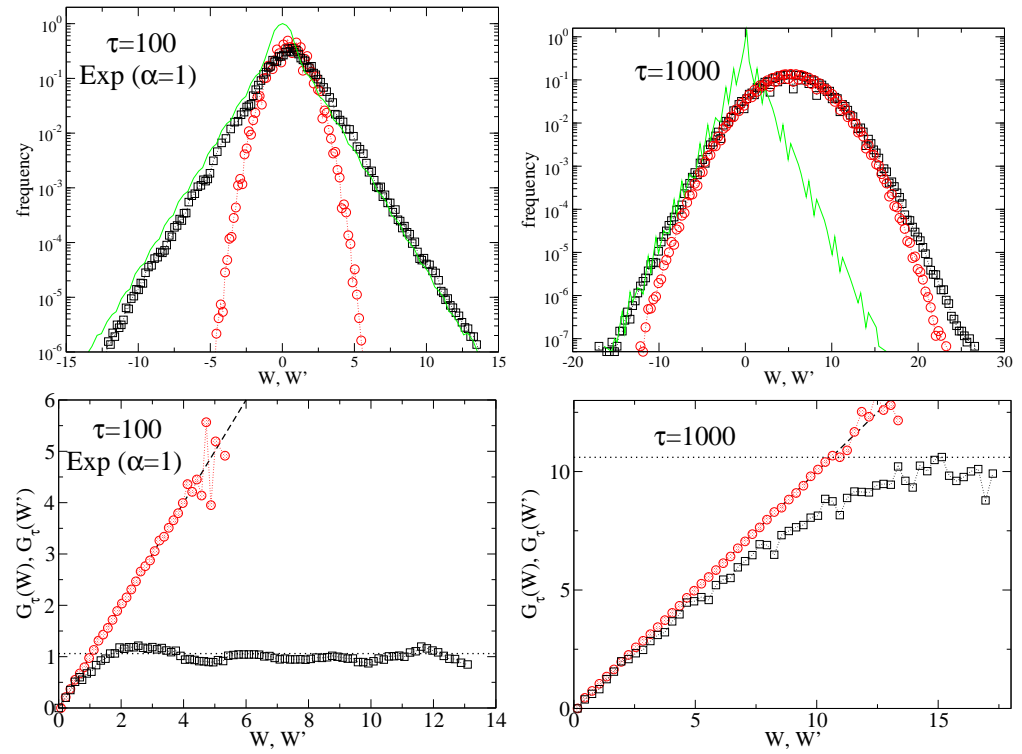

Fig. 16. Study of the boundary terms for the Fluctuation Relations in the Markov chain discussed in the text. Top graphs: probabilities of observing $W$ (black squares), $W^{\prime}$ (red circles) and $B=W^{\prime}-W$ (green lines). Bottom graphs: $G_{\tau}(W)$ vs. $W$ (black squares) and $G_{\tau}\left(W^{\prime}\right)$ vs. $W^{\prime}$ (red circles). The dashed line has slope 1. The dotted horizontal line marks the van Zon and Cohen prediction for the FR violation, $G_{\tau}(W)=2\langle W\rangle$ for large $W[231]$. The left column is at time $\tau=100$ and the right column at time $\tau=1000$. In the simulation we have used $N=50, p_{A A}=0.2$, $p_{A B}=0.3, p_{B A}=0.3$ and $p_{C A}=0.5$ and $k_{i} \propto \exp (-\alpha i)$ with the value of $\alpha$ given in the plot. In all cases $\langle W(\tau)\rangle=\left\langle W^{\prime}(\tau)\right\rangle=0.0053 \tau$, i.e. 0.53 for $\tau=100$ and 5.3 for $\tau=1000$.

\subsection{Numerical works}

An exhaustive account of the numerical works devoted to the study of FRs is practically impossible here, because of space limitations. Therefore, we briefly mention a selected group of works, and we concentrate only on two of them.

The steady state FR for nonequilibrium systems was introduced and numerically verified by Evans et al. [148], in a model in which $\Lambda=\Omega$. After that, some numerical tests have been devoted to the $\Lambda$-FR, in order to understand the ex- 
tent to which the Chaotic Hypothesis and the $\Lambda$-FR describe physical systems, see e.g. $[232,183,233,234,156,235,198,197,236,237,158]$. The result is that the $\Lambda$-FR is easily verified when $\Lambda=\Omega$, while difficulties emerge when this is not the case. The largest group of tests concerns quantities related in various fashions to the entropy production or dissipated energy rates, both in transient and steady states, see e.g. Refs.[232,238,239,183,233,234,154,155,156,240,241]. It has been found that FRs for these physical observables are ubiquitously verified, in models of nonequilibrium fluids.

\subsubsection{The nonequilibrium FPU chain}

Lepri et al. [242] modified the Fermi-Pasta-Ulam model, imposing nonequilibrium boundary conditions by means of two Nosé-Hoover thermostats at different temperatures [238]. This work constitutes a numerical investigation of a steady state FR, meant to test the physical applicability of the relation in a model substantially different from the original one, i.e. in a solid rather than in a fluid. The test of [238] concerned the fluctuations of the heat flux, which verified the FR, supporting the idea that such relations do have a quite wide range of applicability.

The model studied in Ref.[238] consists of $N$ anharmonic oscillators of mass $m$ and positions $x_{j}, j=1, \ldots, N$, whose first and last oscillators $(j=1, N)$ interact with the left $(\ell)$ and the right $(r)$ thermostats, respectively, and with still walls, while all oscillators interact with their nearest neighbours through the potential:

$$
V(q)=\frac{q^{2}}{2}+\beta \frac{q^{4}}{4}
$$

where $q_{j}=x_{j}-j a$ is the displacement of the $j$-th oscillator from its equilibrium position, and $a$ is the equilibrium distance between two nearest neighbours. The equations of motion take the form

$$
\begin{aligned}
& m \frac{d^{2}}{d t^{2}} q_{1}=F\left(q_{1}-q_{0}\right)-F\left(q_{2}-q_{1}\right)-\zeta_{\ell} \frac{d q_{1}}{d t}, \\
& m \frac{d^{2}}{d t^{2}} q_{i}=F\left(q_{i}-q_{i-1}\right)-F\left(q_{i+1}-q_{i}\right), \quad \text { for } i=2, \ldots, N-1 \\
& m \frac{d^{2}}{d t^{2}} q_{N}=F\left(q_{N}-q_{N-1}\right)-F\left(q_{N+1}-q_{N}\right)-\zeta_{r} \frac{d q_{N}}{d t},
\end{aligned}
$$

where $q_{0}=q_{N+1}=0, F=-d V / d z$,

$$
\frac{d}{d t} \zeta_{r}=\frac{1}{\theta_{r}^{2}}\left(\frac{\dot{q}_{N}^{2}}{T_{r}}-1\right), \quad \frac{d}{d t} \zeta_{\ell}=\frac{1}{\theta_{\ell}^{2}}\left(\frac{\dot{q}_{1}^{2}}{T_{\ell}}-1\right),
$$

and $\theta_{r}, \theta_{l}$ are relaxation times which determine the efficiency of the thermostats. In the case that $T_{r} \neq T_{\ell}$, Eqs.(6.33,6.34) define the nonequilibrium 
FPU- $\beta$ model, which is time reversal invariant, with time reversal operation defined by $I(q, p, \zeta)=(q,-p,-\zeta)$. The system is also dissipative, and the time average of the phase space contraction rate, $\left(\bar{\zeta}_{r}+\bar{\zeta}_{\ell}\right)$, is positive.

The authors argue that the heat flux $J$ can be identified, in this case, with the flux of potential energy. Then, they find that the average fluxes at the left, $J_{L}$, and right, $J_{R}$, ends of the chain are given by

$$
J_{L, R}=-\left\langle\zeta_{L, R}\right\rangle T_{L, R}
$$

where $\left\langle\zeta_{L, R}\right\rangle$ is the mean value of the corresponding effective momentum of the thermostat. By subtracting from one another the fluxes in Eq. (6.35) and using the fact $J_{L}=-J_{R}=J$, on average, in the steady state, one obtains

$$
\langle\Lambda\rangle=\left\langle\zeta_{L}\right\rangle+\left\langle\zeta_{R}\right\rangle=\langle J\rangle\left(\frac{1}{T_{R}}-\frac{1}{T_{L}}\right)
$$

This relation is analogous to the one for the global entropy production as obtained from linear response, but while it holds for the averages, the instantaneous values and the fluctuations of $\Lambda$ are not simply proportional to those of $J$. In Ref.[238], the steady state FR for $J$ is tested and found to hold, even though the model does not enjoy normal energy transport, in the large $\mathrm{N}$ limit. This is not a contradiction in itself, although the validity of the FR, close to equilibrium, implies the validity of the Green-Kubo relations (5.61). The reason is that the nonequilibrium FPU chain at finite $N$, enjoys normal transport. The anomalous behaviour is reflected only in the divergence of the heat conductivity with growing $N$.

\subsubsection{Granular materials}

Some difficulties are encountered in experimental or numerical studies of the FR. An example from heated granular gases is discussed here. The theoretical analysis correctly predicts the asymptotic (large deviations, $t \rightarrow \infty$ ) behavior of the fluctuations of the work done on the gas, but measurements done at finite time lead to contrasting conclusions [243,244].

As already discussed in Sect. 4.3 and detailed in the Appendix A, a standard way of modeling a granular gas is to consider a set of $N$ hard-spheres undergoing inelastic collisions, in which a fraction $\left(1-r^{2}\right)$ of the relative kinetic energy is dissipated. The restitution coefficient $r$ lies between 0 and 1, where 1 corresponds to elastic collisions. The inelastic collision rule (A.1) breaks microscopic reversibility, i.e. for any given trajectory in phase space, the time-reversed one is not always physically realizable. One can write an energy balance equation for the total kinetic energy $E(t)=\sum_{i} \frac{1}{2} \mathbf{v}_{i}^{2}$, which 
varies according to

$$
\Delta E=E(t)-E(0)=W(t)-D(t)
$$

where $W(t)$ is the energy injected by an external driving mechanism, while $D(t) \geq 0$ is the energy irreversibly dissipated by the inelastic collisions. The average variation rate of $D$ can be estimated as the collision rate times the energy dissipated through a collision,

$$
\frac{\mathrm{d}}{\mathrm{d} t}\langle D\rangle=-\frac{1-r^{2}}{4 \ell}\left\langle\left|\mathbf{v}_{12} \cdot \boldsymbol{\sigma}\right|^{3}\right\rangle
$$

where $\ell$ is the mean free path, $\boldsymbol{\sigma}$ is the unit vector joining the centers of the colliding particles and $\mathbf{v}_{12}$ is their relative velocity. The mean kinetic energy per particle provides a typical energy scale, also termed granular temperature, and it is defined as

$$
T_{g}=\left\langle\mathbf{v}_{i}^{2}\right\rangle / d=\beta_{g}^{-1},
$$

where $d$ is the spatial dimension. A typical heating mechanism is provided by Eq. (A.2). We discuss here the monodisperse case which achieves a statistically stationary state, thanks to the injection of energy by means of independent random forces acting on each individual particle. This heating mechanism is easier to be handled mathematically and leads to a uniform stationary state. The work provided by the external source reads

$$
W(t)=\sum_{i} \int_{0}^{t} \mathrm{~d} \tau \boldsymbol{\eta}_{i}(\tau) \cdot \mathbf{v}_{i}(\tau)
$$

Given that $\langle W\rangle / t=2 d \Gamma$ (where $\Gamma$ is a parameter of the model, see Appendix A), the typical energy scale is set by Eq. (6.47). Following a procedure similar to that of Section 6.1.1, we compute the large deviation function for the injected work $W$.

We begin introducing a phase space density $\rho(\mathbf{P}, W, t)$ that counts the number of systems in state $\mathbf{P}$ (the $N$-velocities phase space point) which, in the time window $[0, t]$, accumulated a total work $W(t)=W$. A generalized Liouville equation can be written for $\rho$, in which the Liouville operator $\mathcal{L}_{W}$ can be split into a $W$ conserving part, $\mathcal{L}_{\text {coll }}$, and a part accounting for changes in $W$ under the effect of the external injection mechanism $\mathcal{L}_{\text {inj }}$ :

$$
\partial_{t} \rho=\mathcal{L}_{W}(\mathbf{P}, W) \rho=\mathcal{L}_{\text {inj }}(\mathbf{P}, W) \rho+\mathcal{L}_{\text {coll }}(\mathbf{P}) \rho
$$

It is convenient to introduce the Laplace transform of $\rho$,

$$
\hat{\rho}(\mathbf{P}, \lambda, t)=\int \mathrm{d} W \mathrm{e}^{-\lambda W} \rho(\mathbf{P}, W, t),
$$

and rewrite the Liouville equation in terms of $\hat{\rho}$,

$$
\partial_{t} \hat{\rho}=\mathcal{L}_{W}(\mathbf{P}, \lambda) \hat{\rho}=\mathcal{L}_{\text {inj }}(\mathbf{P}, \lambda) \hat{\rho}+\mathcal{L}_{\text {coll }}(\mathbf{P}) \hat{\rho}
$$


The largest eigenvalue $\mu(\lambda)$ of $\mathcal{L}_{W}(\lambda)$ governs the asymptotic behavior of $\hat{\rho}$,

$$
\hat{\rho}(\mathbf{P}, \lambda, t) \simeq C(\lambda) \mathrm{e}^{\mu(\lambda) t} \tilde{\rho}(\mathbf{P}, \lambda)
$$

where $\tilde{\rho}(\mathbf{P}, \lambda)$ is the (right) eigenvector of $\mathcal{L}_{W}(\mathbf{P}, \lambda)$, associated with $\mu(\lambda)$, and $C(\lambda)$ is the projection of the initial state on this eigenvector. It is then possible to project on the single-particle distribution $f^{(1)}(\mathbf{v}, \lambda, t)=\int d \mathbf{P}_{N-1} \hat{\rho}(\mathbf{P}, \lambda, t)$. We arrive at

$$
\partial_{t} f^{(1)}(\mathbf{v}, \lambda, t)=\Gamma \Delta_{\mathbf{v}} f^{(1)}+2 \lambda \Gamma \mathbf{v} \cdot \mathbf{v} f^{(1)}+\Gamma\left(\lambda^{2} v^{2}-d \lambda\right) f^{(1)}+\text { Coll. }
$$

where in the rhs of (6.45) "Coll" is a shorthand for the collision operator which, resorting to the molecular chaos hypothesis, reads

$$
\text { Coll. }=\frac{1}{\ell} \int_{\mathbf{v}_{12} \cdot \boldsymbol{\sigma}>0} \mathrm{~d} \mathbf{v}_{2} \mathrm{~d} \boldsymbol{\sigma}\left(\mathbf{v}_{12} \cdot \boldsymbol{\sigma}\right)\left(\frac{1}{r^{2}} f^{(1)}\left(\mathbf{v}_{1}^{*}, \lambda\right) f^{(1)}\left(\mathbf{v}_{2}^{*}, \lambda\right)-f^{(1)}\left(\mathbf{v}_{1}, \lambda\right) f^{(1)}\left(\mathbf{v}_{2}, \lambda\right)\right),
$$

where $\mathbf{v}_{1}^{*}$ and $\mathbf{v}_{2}^{*}$ are the pre-collisional velocities, defined in the Appendix A. The steady velocity pdf equation (Boltzmann equation) is recovered at $\lambda=0$. The process encoded in (6.45) can be read off as the original granular gas dynamics in which additional particles are created when $\lambda>0$ or annihilated when $\lambda<0$, at a velocity dependent rate $\lambda v^{2}$. The largest eigenvalue of $\mathcal{L}_{W}(\lambda)$, $\mu(\lambda)$ is then interpreted as the population growth rate. This remark was numerically exploited, for somewhat different systems in [245]. The splitting of $f^{(2)}$ as a product of independent one particle distributions is indeed a kind of molecular chaos hypothesis, for the system with particle non-conserving fictitious dynamics. The Boltzmann equation toolbox offers many ways to deduce an expression for $\mu(\lambda)$. The simplest approximation is to project $\tilde{f}^{(1)}(\mathbf{v}, \lambda)$ onto a Gaussian with a $\lambda$-dependent variance, denoted as $T(\lambda)$, as explained in [244].

Working in terms of rescaled quantities, with the granular temperature precisely given by

$$
T_{g}=\left(\frac{2 d \ell \Gamma \sqrt{\pi}}{\left(1-r^{2}\right) \Omega_{d}}\right)^{2},
$$

with $\Omega_{d}=2 \pi^{d / 2} / \Gamma(d / 2)$, and performing the following substitutions

$$
\mu \rightarrow \mu \frac{T_{g}}{d \Gamma N}, \quad \lambda \rightarrow \lambda T_{g}
$$

one has:

$$
\mu(\lambda)=-\lambda+\frac{1}{2} \frac{T(\lambda)}{T_{g}} \lambda^{2}
$$

for the dimensionless quantities, where $\sqrt{T(\lambda)}$ sets the typical velocity scale for trajectories characterized by $\lambda$. At large values of $\lambda$, corresponding to values of $W$ small with respect to $\langle W\rangle$, we expect that $T(\lambda) \ll T_{g}$ and indeed $\frac{T(\lambda)}{T_{g}} \simeq \frac{2}{\lambda}$ 


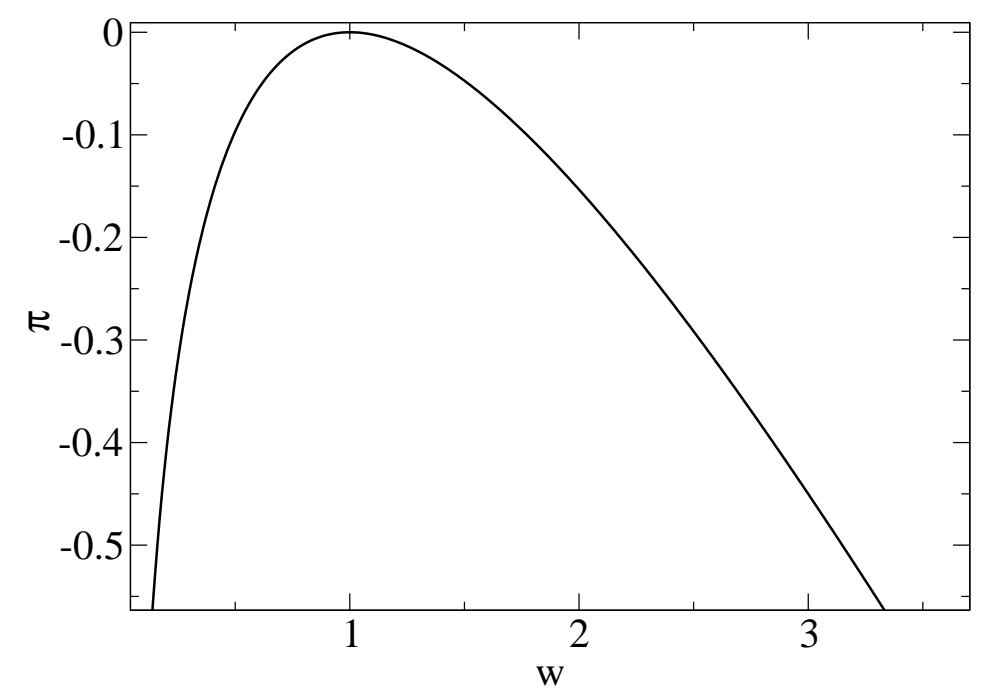

Fig. 17. Plot of the Cramer function for the injected power $\pi(w)=\lim _{t \rightarrow \infty} \frac{1}{t} \log f(t, w t)$, where $f(t, W)$ is the pdf of $W$ at time $t$.

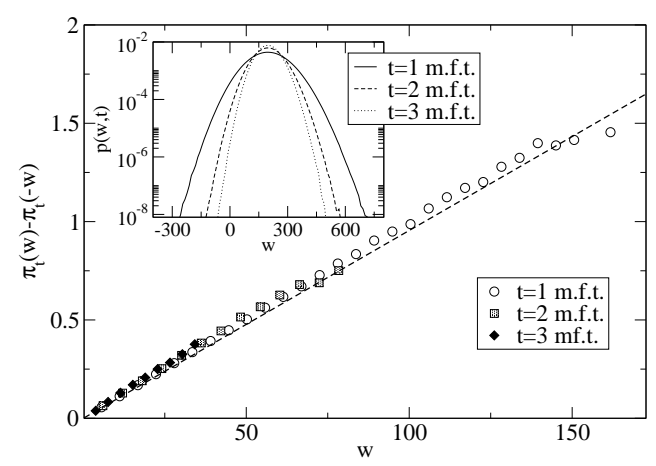

Fig. 18. Plot of $\pi(w, t)-\pi(-w, t)$ for $t=1,2$ and 3 mean free times (mft). The inset shows the probability density function of $w(t)$ for the same times.

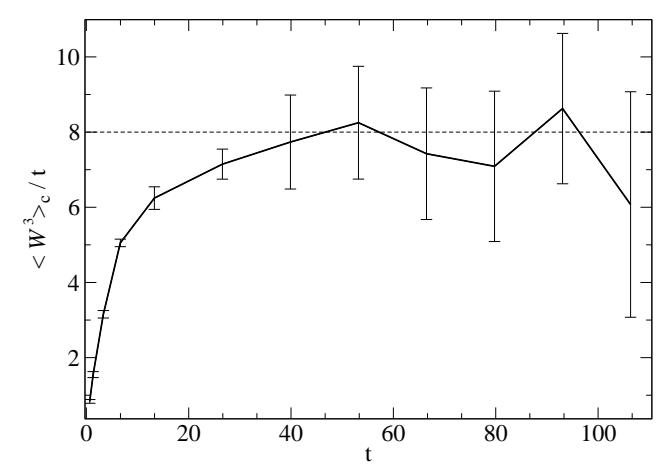

Fig. 19. Plot of the third cumulant of $W$ divided by time. The asymptotic regime is reached after fifty mft.

as $\lambda \rightarrow+\infty[243,244]$. This can be further refined to obtain the behavior of $\mu(\lambda)$ at $\lambda \rightarrow+\infty$,

$$
\mu(\lambda) \simeq-\lambda^{1 / 4}
$$

Besides, $\mu(\lambda)$ possesses a cut in the $\lambda$-plane at $\lambda_{c}=-3 / 2^{8 / 3}$, such that, as $\lambda \rightarrow \lambda_{c}^{+}$,

$$
\mu(\lambda)=3 / 2^{2 / 3}-3^{3 / 2} 2^{1 / 6} \sqrt{\lambda-\lambda_{c}}+\mathcal{O}\left(\lambda-\lambda_{c}\right)
$$

The presence of this cut is responsible for the exponential decay of the pdf of $W$ at large values of $W$. However, the non-analytic behavior at $\lambda \rightarrow+\infty$ leads to a non-analytic behavior as $w=W / t \rightarrow 0^{+}$,

$$
\pi\left(w \rightarrow 0^{+}\right) \sim-w^{-1 / 3}, \quad \pi(w \rightarrow\langle w\rangle) \simeq-(w-1)^{2} / 2, \quad \pi(w \rightarrow \infty) \sim-w .
$$


Simulations of the granular gas heated with the stochastic thermostat $[243,244]$ confirm the above results and thus the validity of these approximations. The total work $W(t)$ provided by the random forces over $[0, t]$ is of course, on average, a positive quantity, but at finite times there exist phase space trajectories which yield a negative $W$. However, one of the key hypotheses underlying the Fluctuation Relation is that the dynamics features at least a weak form of time reversibility, for which trajectories (or ensembles of trajectories) possess time reversed counterparts (however unlikely). In our granular gas, dissipative collisions prevent time reversed trajectories to be physically acceptable trajectories at all. It should therefore be no surprise that no specific fluctuation relation holds in our case. This is confirmed by the explicit calculations presented above, that show no specific symmetry property of the injected power large deviation function. In spite of this, it may be instructive to plot $w$ vs. $\frac{1}{t} \ln \frac{P(w t, t)}{P(-w t, t)}$ at possibly large times, since this is exactly $\pi(w)-\pi(-w)$, being $\pi(w, t)=\frac{1}{t} \ln P(w t, t)$, in the infinite time limit. This is shown in figure 18: one actually observes a straight line with slope $\beta_{g}$, while the analytic results suggest the absence of such a straight line, even for values of $w$ not too far from its average. In the first place, a numerical simulation is always carried out for finite times, and thus one measures $\pi(w, t)$ rather than its $t \rightarrow \infty$ limit, $\pi(w)$. Though $\pi(w, t)$ deviates from a quadratic form, which would correspond to Gaussian $P(W, t)$, this is no proof that the asymptotic regime has been reached. For that matter it is instructive to investigate the behavior of the third cumulant $\left\langle W^{3}\right\rangle / t$ as a function of time, presented in figure 19. The conclusion is that the third cumulant reaches its asymptotic value, consistent with the analytic expression, at times a few tens as large as those for which negative values of $w=W / t$ can be measured, as shown in figure 18. A similar plot of the fourth cumulant would signal that the asymptotic regime has not been reached over the chosen time window. What one actually observes is simply the remnant of a short time quadratic behavior for $\pi(w)$. This is also consistent with the quadratic approximation for $\mu(\lambda)$, which would indeed imply $\pi(w)-\pi(-w)=\beta_{g} w$, after restoring the appropriate physical scales as in (6.48).

\subsection{Experimental works}

Experimental tests of FRs are usually rather delicate, because large fluctuations are not directly observable in macroscopic systems. Therefore, one must either rely on the validity of local versions of the FRs, or consider small systems, like micro-biological systems or nano-technological devices. Some experimental works on the FRs, and on similar relations are the following:

- Ciliberto and Laroche's experiment [246], for the temperature fluctuations in a fluid undergoing Rayleigh-Bénard convection. A linear law was observed, 
but with a different (perhaps not asymptotic) slope.

- Wang et al. experiments, for the transient and steady state fluctuations of work done on a colloidal particle, dragged through water by means of an optical trap, [165,247]. These experiments concerned nanoscale systems and motivated Ref.[165]

- Feitosa and Menon's experiment on a mechanically driven inelastic granular gas in a fluidized steady state [248]. They claimed a verification of a local version of FR, but their analysis has been criticized (cf. Section 6.3.1).

- Garnier and Ciliberto's experiment on the fluctuations of the dissipated power of an electric dipole [249]. They considered the work and heat fluctuations, both related with the fluctuations of the power dissipated by the resistor, and partly verified the Van Zon - Cohen scenario.

- Shang et al. experiment on the fluctuations of a local entropy production in turbulent thermal convection [250]. They considered a cylindrical cell filled with water of aspect ratio one, and verified the steady stat FR.

- Ciliberto et al. experiment on turbulent flows, verified the local version of the FR, proposed in $[182,183]$

- Douarche et al. experiment on the steady state and transient work fluctuations of a damped harmonic oscillator, kept out of equilibrium by an external force [251]. The transient FR was confirmed for any averaging time of the work. The steady-state version was observed to converge to the asymptotic behaviour.

- Tietz et al. experiment on the entropy production in a single two-level system, a defect center in natural IIa-type diamond [252].

It must be noted that almost all of these experiments afford verifications of the FRs only to a certain degree, because various parameters of the relevant theories are not accessible or controllable in them. Some authors even argue that a direct verification of the (steady state) FRs may be practically impossible [253]. This opinion is likely too extreme, but the difficulty, so far, remains. Therefore, the observation of any approximately linear arrangement of data, with any slope, is often taken as sufficient evidence for the validity of the steady state relation, even if obtained for relatively short averaging times. Easier is the case of the transient relations. Because these relations are algebraic "identities", which hold under almost no conditions, one may find odd that they are tested all, but there are at least two reasons for doing it. The first is to verify the relevance of the models for which these relations have been derived, to the physical reality. Indeed, those models have been devised and proved to be successful for certain purposes which, in general, are not those of the FRs, as recalled in Subsection 5.2. The second reason is that one can still rely on a few quantitative theoretical results, for the interpretation of certain nonequilibrium phenomena (protein stretching experiments, etc.).

Despite the difficulty in gathering the necessary statistics, the transient relations offer some of the few parameter-free quantitative predictions, which may 
be used to infer equilibrium properties of certain systems, from nonequilibrium experiments. The Jarzynski and Crooks relations, in particular, have been tested in a good number of experiments including molecular and biophysical experiments. The reason is that this equality can be used to estimate the equilibrium free energy out of measurements of dissipated work in nonequilibrium processes. This is particularly useful in systems for which no other method to estimate the free energy exists. Douarche et al. experimentally confirmed both in [254]. An experiment for the colloidal particle in a time-dependent non-harmonic potential was reported by Blickle et al. [255]. The Jarzynski equality was first confirmed in 2002 by Liphardt et al. in measurements of the dissipated work in the folding-unfolding process of a single molecule of RNA [256]. Collin et al. experimentally confirmed the Crooks fluctuation relation by pulling an RNA hairpin [257]. As discussed in detail in 6.3.2, using optical tweezers they measured the fluctuations of the dissipative work during the unfolding and refolding of a small RNA molecule. Their measurements confirmed the Crooks relation near and far from equilibrium. They also estimated the free energy from the nonequilibrium measurements. The nonequilibrium steady-state equality of Hatano and Sasa [220] has been verified by Trepagnier et al. [258]. A detailed discussion of these experiments can be found in the review papers [259] and [142].

\subsubsection{Experiments with granular gases}

Recently an experiment has been performed by Menon and Feitosa [248,260] using a granular gas shaken in a container at high frequency. The setup consisted of a $2 D$ box containing $N$ identical glass beads, vibrated at frequency $f$ and amplitude $A$. The authors observed the kinetic energy variations $\Delta E_{\tau}$, over time windows of duration $\tau$, in a central sub-region of the system characterized by an almost homogeneous temperature and density. They subdivided this variation into two contributions:

$$
\Delta E_{\tau}=W_{\tau}-D_{\tau}
$$

where $D_{\tau}$ is the energy dissipated in inelastic collisions and $W_{\tau}$ is the energy flux through the boundaries, due to the kinetic energy transported by incoming and outgoing particles. The authors of the experiment have conjectured that $W_{\tau}$, being a measure of injected power in the sub-system, can be related to the entropy flow or the entropy produced by the thermostat constituted by the rest of the gas (which is equal to the internal entropy production in the steady state). They have measured its pdf $f\left(W_{\tau}\right)$ and found that

$$
\ln \frac{f\left(W_{\tau}\right)}{f\left(-W_{\tau}\right)}=\beta W_{\tau}
$$


with $\beta \neq 1 / T_{g}$. By lack of a reasonable explanation for the value of $\beta$, the authors have concluded to have experimentally verified the FR with an "effective temperature" $T_{\text {eff }}=1 / \beta$, suggesting its use as a possible non-equilibrium generalization of the usual granular temperature. The same results have been found in MD simulations of inelastic hard disks with a similar setup, see Figure 20. There is a major objection to this reasoning: in the limit of zero inelasticity (i.e. in the case of ideal elastic grains) the pdf $f\left(W_{\tau}\right)$ becomes symmetric with respect to the average injected power through the borders of the central region, which is 0 , so that $\beta \rightarrow 0$ and the effective temperature would diverge instead of coinciding with the equilibrium temperature. There is also a subtler problem in the interpretation of this experiment: from Equation (6.53), it can be argued that the large deviation function of $f\left(W_{\tau}\right)$ equals the large deviation function of $f\left(D_{\tau}\right)$. Recall that $f\left(W_{\tau}\right) \sim \exp \left(\tau \pi\left(W_{\tau} / \tau\right)\right)$ when $\tau \rightarrow \infty$, and that $\pi(q)$ is the large deviation function of $f\left(W_{\tau}\right)$, with $q=W_{\tau} / \tau$. Since $D_{\tau}$ is always positive, it follows that the relation (6.54) cannot hold for $\tau \rightarrow \infty$.
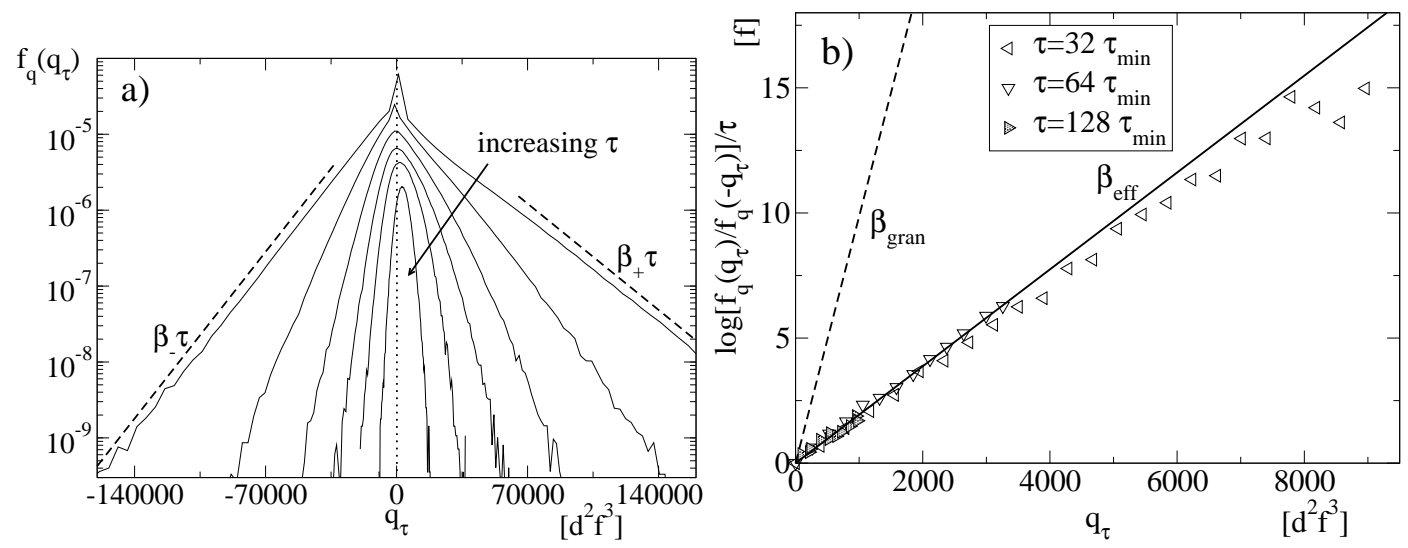

Fig. 20. a) pdfs of injected power $f_{q}\left(q_{\tau}\right)$ from MD simulations for different values of $\tau=(1,2,4,8,16,32) \times \tau_{\text {min }}$ with $\tau_{\text {min }}=0.015 \tau_{\text {box }}$. Here $N=270$ and $\alpha=0.9$. The distributions are shifted vertically for clarity. The dashed lines put in evidence the exponential tails of the pdf at $\tau=\tau_{\min }$. b) plot of $(1 / \tau) \log \left(f_{q}\left(q_{\tau}\right) / f_{q}\left(-q_{\tau}\right)\right)$ vs. $q_{\tau}$ from MD simulations (same parameters as above) at large values of $\tau$. The solid curve is a linear fit (with slope $\beta_{\text {eff }}$ ) of the data at $\tau=128 \tau_{\min }$. The dashed line has a slope $\beta_{\text {gran }}=1 / T_{\text {gran }}$

A different explanation has been proposed in [260], which does not involve the FR but rather imputes the observed results to effects of finite time and poor statistics due to the limits of the measurement procedure. It appears that the injected power measured in the experiment can be written as

$$
W_{\tau}=\frac{1}{2}\left(\sum_{i=1}^{n_{+}} v_{i+}^{2}-\sum_{i=1}^{n_{-}} v_{i-}^{2}\right),
$$

where $n_{-}$(resp. $n_{+}$) is the number of particles leaving (resp. entering) the subregion during the time $\tau$ (with frequency $\omega$ ), and $v_{i-}^{2}\left(v_{i+}^{2}\right)$ are the squared moduli of their velocities. In order to analyze the statistics of $W_{\tau}$ we take 
$n_{-}$and $n_{+}$as Poisson-distributed random variables with average $\omega \tau$, neglecting correlations among particles entering or leaving successively the central region. As supported by the direct observation of the simulation [260], the velocities $\mathbf{v}_{i+}$ and $\mathbf{v}_{i-}$ are assumed to originate from two distinct populations with different temperatures $T_{+}$and $T_{-}$respectively. Indeed, compared with the population entering the central region, the particles that leave it have suffered on average more inelastic collisions, so that $T_{-}<T_{+}$. Finally Gaussian velocity pdfs 22 are assumed. This allows one to calculate the generating function of $f\left(W_{\tau}\right)$, obtaining $g(z)=\exp (\tau \mu(z))$ for any $\tau$, with

$$
\mu(z)=\omega\left(-2+\left(1-T_{+} z\right)^{-D / 2}+\left(1+T_{-} z\right)^{-D / 2}\right),
$$

which also automatically coincides with the large deviation function of $g(z)$. We recall that the two large deviation functions $\mu(z)$ and $\pi(q)$ are related by a Legendre transform. A FR with a slope $\beta$ implies that $\mu(z)=\mu(\beta-z)$ and this relation is not fulfilled by Eq. (6.56). It has, however, been observed [261] that $\pi(q)-\pi(-q) \approx 2 \pi^{\prime}(0) q+o\left(q^{3}\right)$ at small values of $q$. This means that the linear relation (6.54) with $\beta_{\text {eff }}=2 \pi^{\prime}(0)$ can be observed if the range of $W_{\tau}$ is small, which is usually the case, since in experiments and simulations the statistics of negative events is very poor. From equation (6.56) a formula for the slope $\beta$ follows:

$$
\beta_{\text {eff }}=2 \frac{\gamma^{\delta}-1}{\gamma+\gamma^{\delta}} \frac{1}{T_{-}}
$$

with $\gamma=T_{+} / T_{-}$and $\delta=2 /(2+d)$. When $\gamma=1$ (i.e. when the gas is elastic) $\beta_{\text {eff }}=0$. This formula is in good agreement with the slope observed in the simulations and in the experiment.

The fact that expression (6.57) is in good quantitative agreement with the experiment is consistent with the observation of negative events, i.e. not too large $\tau$. We can thus argue that the experiment and the simulations have been performed over times large enough to test the validity of formula (6.57), but not so large that correlations may be disregarded.

\subsubsection{Applications of the Jarzynski relation to biophysical systems}

The second principle of thermodynamics implies that the free energy difference $F(B)-F(A)$ between two equilibrium states $A$ and $B$ is smaller or equal than the work $W_{\tau}$ done by the external force over the system during the $A \rightarrow B$ transformation taking a time $\tau$ :

$$
W_{\tau} \geq \Delta F=F(B)-F(A) .
$$

$\overline{22}$ while non-Gaussian tails are quite common in granular gases, it has been checked that they play a negligible role here 
The equal sign applies only to infinitely slow transformations, i.e. $\tau \rightarrow \infty$. For any finite $\tau$, even averaging over many realizations, say $M$, of the same experiment $A \rightarrow B$, the measured $\left\langle W_{\tau}\right\rangle=\frac{1}{M} \sum_{i=1}^{M} W_{\tau, i}$ is bound by the second principle to be larger than $\Delta F$.

The Jarzynski equality (5.62) allows to measure $\Delta F$ by means of non-adiabatic transformations $A \rightarrow B$, provided that the average $\left\langle W_{\tau}\right\rangle$ is replaced by a weighted average $\left\langle W_{\tau}\right\rangle_{J}$, i.e. the equal sign is always true in $\Delta F=\left\langle W_{\tau}\right\rangle_{J}$ with

$$
\left\langle W_{\tau}\right\rangle_{J}=-\beta^{-1} \ln \left\langle\exp \left(-\beta W_{\tau}\right)\right\rangle \approx-\beta^{-1} \ln \left[\frac{1}{M} \sum_{i=1}^{M} \exp \left(-\beta W_{\tau, i}\right)\right] .
$$

As noticed in Section 5.4.3, this argument reverses the link between equilibrium and non-equilibrium phenomena implied by the classical FDR. For instance, Green-Kubo formulas give a practical recipe to calculate transport coefficients from correlation functions in the unperturbed system, i.e. they extract non-equilibrium properties from equilibrium ones. On the contrary, in the case of Eq. (6.59), an equilibrium property, $\Delta F$, is obtained by means of non-equilibrium measurements. This is particularly useful in biology, where the reconstruction of the free-energy landscape of (large) complex molecules, such as proteins is crucial for determining folding pathways, the topology of the folded state, and the biological utility of the protein. The speed of a fair realization of reversibility along a reaction coordinate is determined by the relaxation time of the protein, which can be very long: therefore a naive estimate of $\left\langle W_{\tau}\right\rangle$ would require an exceedingly large $\tau$. Before the Jarzynski relation, the only known correction to the rough estimate $\left\langle W_{\tau}\right\rangle$ was $[262]$

$$
\Delta F \approx \Delta F_{F D R}=\left\langle W_{\tau}\right\rangle-\beta \sigma^{2} / 2
$$

with $\sigma^{2}$ the variance of $W_{\tau}$, which is valid only near the reversible path, i.e. still for large $\tau$. This correction can be obtained from the FDR for Hamiltonian systems. A posteriori, from the Jarzynski relation, Eq. (6.60) is easily obtained, assuming that the distribution of $W_{\tau}$ is Gaussian.

The alternative provided by Eq. (6.59), suggested in [263] and experimentally tested in [256] for the first time, consists in performing $M$ realizations of a pulling experiment on a single bio-molecule: the external force, provided by the cantilever of an Atomic Force Microscopy or by moving the laser trap of an optical tweezer (this was done in the real experiment), is applied to a terminal of the molecule, through a bead that plays the role of a handle, guiding the transformation from a folded $A$ to an unfolded $B$ state in a time $\tau$. Measures in numerical and real experiments have shown that the weighted average (6.59) can be effectively used to have a better estimate of $\Delta F$ with respect to $\Delta F_{F D R} \mathrm{Eq}$. (6.60), for almost the whole range of displacements 
between $A$ and $B$. Actually the applicability of Eq. (6.59) is not trivial, since a careful evaluation of all possible errors is required: reducing $\tau$ allows to increase the number of experiments $M$, but at the same time makes wider and wider the distribution of $W_{\tau}$ and, therefore, more and more uncertain the estimate of the exponential average $\left\langle\exp \left(-\beta W_{\tau}\right)\right\rangle$ through a finite sum. However it has been shown $[264,265]$ that a range of $\tau$ exists where the estimate is reliable. Moreover, this procedure has the advantage of providing a direct estimate of the statistical error of the measure, which is not possible with a small number of measurements and large $\tau$. On the other side, when applying a similar procedure to large systems, the difficulty arises that sampling negative $W_{\tau}$ requires the number of independent experiments $M$ to grow exponentially with the system size [266].

\section{Large deviations and FR}

In Sections 2, 3 and 4 we discussed the properties of the mean responses, and their link with correlation functions. Sections 5 and 6 have been devoted to a more detailed statistical description of the probability distribution of time averages on certain intervals. Now, we want to treat at a more advanced level the statistical description of the time history of the system, as described by its observables.

\subsection{Onsager-Machlup approach to fluctuations of thermodynamic variables}

As far as we know the first attempt in this direction was made by Onsager and Machlup, about half a century ago [267,268]. In two influential papers they studied the probability of a given succession of nonequilibrium states, of a spontaneously fluctuating thermodynamic system, under the assumption that the $N$ variables $\{\alpha\}$ determining the state are Gaussian stochastic processes. Basically, Onsager and Machlup assume that the evolution of $\{\alpha\}$ is determined by a set of linear Langevin equations:

$$
\sum_{j=1}^{N} R_{i, j} \frac{d \alpha_{j}}{d t}=-\sum_{j=1}^{N} s_{i, j} \alpha_{j}+\sqrt{2 c} \eta_{i}
$$

where $\{\eta\}$ is a white noise vector, i.e. a Gaussian process with $<\eta_{k}(t)>=0$ and $<\eta_{k}(t) \eta_{j}\left(t^{\prime}\right)>=\delta\left(t-t^{\prime}\right) \delta_{k, j}$. The symmetric matrices $\mathbf{R}=\left\{R_{i, j}\right\}$ and $\mathbf{s}=\left\{s_{i, j}\right\}$ determine the thermodynamic forces $\{X\}$ and the entropy $S(\{\alpha\})$ :

$$
X_{i}=\frac{\partial S}{\partial \alpha_{i}}=-\sum_{j=1}^{N} s_{i, j} \alpha_{j}
$$


In this approximation, one can express the entropy as

$$
S(\{\alpha\})=S_{0}-\frac{1}{2} \sum_{i, j} s_{i, j} \alpha_{j} \alpha_{i}
$$

whereas the dissipation function is

$$
\phi=\frac{1}{2} \sum_{i, j} R_{i, j} \frac{d \alpha_{i}}{d t} \frac{d \alpha_{j}}{d t}
$$

and the corresponding entropy functional is

$$
\psi=\frac{1}{2} \sum_{i, j} L_{i, j} X_{i} X_{j}
$$

where the matrix $\mathbf{L}$ is defined by $\mathbf{L X}=d \boldsymbol{\alpha} / d t$ i.e. $\mathbf{L}=\mathbf{R}^{-1} 23$ Because of the Onsager reciprocal relations, the matrices $\mathbf{L}, \mathbf{R}$ and $\mathbf{s}$ are symmetric (see section 2.4.3), and the Boltzmann principle implies that the invariant probability distribution is given by

$$
P_{i n v}(\{\alpha\}) \propto \exp [S(\{\alpha\})] \propto \exp \left[-\frac{1}{2} \sum_{i, j} s_{i, j} \alpha_{j} \alpha_{i}\right]
$$

where we took $k_{B}=1$. The value of $c$ in (7.1) is determined in such a way that Eq. (7.6) holds. Above we have used the original notation of the paper by Onsager and Machlup. In modern notation one would have

$$
\frac{d \alpha_{k}}{d t}=-\sum_{j=1}^{N} B_{j, k} \alpha_{j}+\sqrt{2 c} \tilde{\eta}_{k} \quad k=1, \ldots, N
$$

where now $\{\tilde{\eta}\}$ is a Gaussian process with $<\tilde{\eta}_{k}(t)>=0$ and $<\tilde{\eta}_{k}(t) \tilde{\eta}_{j}\left(t^{\prime}\right)>=$ $C_{k, j} \delta\left(t-t^{\prime}\right)$. The link with the original notation, is given by $\mathbf{B}=\mathbf{R}^{-1} \mathbf{s}$ and $\mathbf{C}=\mathbf{R}^{-2}$.

Let, for the sake of simplicity, $N=1$ :

$$
\frac{d \alpha}{d t}=-\gamma \alpha+\sqrt{2 c} \eta
$$

with $\gamma=s / R$. The conditional probability density for $\alpha\left(t_{1}\right)=\alpha_{1}$, given $\alpha\left(t_{0}\right)=\alpha_{0}$, is

$$
P_{1}\left(\alpha_{1}, t_{1} \mid \alpha_{0}, t_{0}\right)=\frac{1}{\sqrt{2 \pi C\left(1-a(\tau)^{2}\right)}} \times \exp \left(-\frac{\left(\alpha_{1}-a(\tau) \alpha_{0}\right)^{2}}{2 C\left[1-a(\tau)^{2}\right]}\right)
$$

$\overline{23}$ As stressed by many authors, e.g. ([267]), a thermodynamic description of a system requires an answer to the general question "how do you know you have taken enough variables, for it to be Markovian?" For instance a noisy $R C$ circuit is Markovian in the variables $(q, \dot{q})$ but not for $q$ (or $\dot{q}$ ) alone. 
where $t_{1}=t_{0}+\tau$ and

$$
a(\tau)=e^{-\gamma \tau}, \quad C=\frac{c}{\gamma}=\frac{1}{s} .
$$

Using the Markovian nature of the process, it is easy to determine the probability density of a discrete trajectory, $\alpha\left(t_{1}\right)=\alpha_{1}, \alpha\left(t_{2}\right)=\alpha_{2}, \ldots \alpha\left(t_{n}\right)=$ $\alpha_{n} \ldots \alpha\left(t_{M}\right)=\alpha_{M}$, where $t_{n}=t_{0}+n \tau$ and $\alpha\left(t_{0}\right)=\alpha_{0}$. One gets

$$
P_{M}\left(\alpha\left(t_{1}\right)=\alpha_{1}, \ldots, \alpha\left(t_{M}\right)=\alpha_{M} \mid \alpha_{0}, t_{0}\right)=\prod_{n=1}^{M} P_{1}\left(\alpha_{n}, t_{n} \mid \alpha_{n-1}, t_{n-1}\right) .
$$

In the limit of small $\tau$, one obtains the rather transparent expression:

$$
P_{1}\left(\alpha_{n}, t_{n}=t_{n-1}+\tau \mid \alpha_{n-1}, t_{n-1}\right)=\frac{1}{\sqrt{4 \pi D \tau}} \times \exp \left[-\ell\left(\alpha_{n}, \alpha_{n-1}\right) \tau\right]
$$

where

$$
\ell\left(\alpha_{n}, \alpha_{n-1}\right)=\frac{1}{4 D}\left[\frac{\left(\alpha_{n}-\alpha_{n-1}\right)}{\tau}+\gamma \alpha_{n}\right]^{2}, \text { with } D=C \gamma=\frac{1}{R},
$$

which leads to

$P_{M}\left(\alpha\left(t_{1}\right)=\alpha_{1}, \ldots, \alpha\left(t_{M}\right)=\alpha_{M} \mid \alpha_{0}, t_{0}\right)=\left(\frac{1}{4 \pi D \tau}\right)^{M / 2} \times \exp \left[-\sum_{n=1}^{M} \ell\left(\alpha_{n}, \alpha_{n-1}\right) \tau\right]$.

The conditional probability distribution $P_{1}\left(\alpha_{M}, t_{M} \mid \alpha_{0}, t_{0}\right)$ can be obtained from (7.13), integrating over $\alpha_{1}, \alpha_{2}, \ldots, \alpha_{M-1}$. Performing the limit $\tau \rightarrow 0$ and $M \rightarrow \infty$ in such a way that $\tau M=$ constant $=t-t_{0}$, one obtains

$$
P_{1}\left(\alpha(t), t \mid \alpha\left(t_{0}\right), t_{0}\right)=\int_{\alpha\left(t_{0}\right)}^{\alpha(t)} \mathcal{D} \alpha\left(t^{\prime}\right) \exp \left\{-\int_{t_{0}}^{t} d t^{\prime} \mathcal{L}\left[\alpha\left(t^{\prime}\right), \dot{\alpha}\left(t^{\prime}\right)\right]\right\}
$$

where the Lagrangian function is

$$
\mathcal{L}[\alpha, \dot{\alpha}]=\frac{1}{4 D}(\dot{\alpha}+\gamma \alpha)^{2}
$$

and the functional integral is defined in the usual way, i.e.

$$
\int_{\alpha\left(t_{0}\right)}^{\alpha(t)} \mathcal{D} \alpha\left(t^{\prime}\right) X_{t}\left[\left\{\alpha\left(t^{\prime}\right)\right\}\right]=\lim _{M \rightarrow \infty}\left(\frac{1}{4 \pi D \tau}\right)^{M / 2} \int d \alpha_{1} \int, \ldots, \int d \alpha_{M} X_{t}\left[\left\{\alpha\left(t_{1}\right), \ldots,\left\{\alpha\left(t_{M}\right)\right]\right.\right.
$$

for any functional $X_{t}\left[\left\{\alpha\left(t^{\prime}\right)\right\}\right]$.

The probability density of a path $\{\alpha(t)\}$ is

$$
P\left(\left\{\alpha\left(t^{\prime}\right)\right\}, t_{0}<t^{\prime}<t\right) \sim \exp \left\{-\int_{t_{0}}^{t} d t^{\prime} \mathcal{L}\left[\alpha\left(t^{\prime}\right), \dot{\alpha}\left(t^{\prime}\right)\right]\right\} .
$$


Noting that

$$
\mathcal{L}[\alpha, \dot{\alpha}]=\frac{R}{4}(\dot{\alpha}+\gamma \alpha)^{2}=\frac{1}{2}\left(\phi+\psi-\frac{d S}{d t}\right)
$$

one can recast (7.17) into

$$
P\left(\left\{\alpha\left(t^{\prime}\right)\right\}, t_{0}<t^{\prime}<t\right) \sim \exp \left\{-\frac{1}{2} \int_{t_{0}}^{t} d t^{\prime}(\phi+\psi)+\frac{1}{2}\left[S\left(\alpha_{t}\right)-S\left(\alpha_{0}\right)\right]\right\} .
$$

This result plays a role analogous to the Boltzmann principle in nonequilibrium statistical mechanics because it expresses the probability of a state in terms of its entropy. Here the probability of a sequence of states is expressed in terms of the entropy and of the dissipation function.

The most probable value of $\alpha(t)$ is obtained minimizing $\int_{t_{0}}^{t} d t^{\prime} \mathcal{L}\left[\alpha\left(t^{\prime}\right), \dot{\alpha}\left(t^{\prime}\right)\right]$ :

$$
\frac{d \alpha}{d t}=-\gamma \alpha \text { with } \alpha\left(t_{0}\right)=\alpha_{0}
$$

i.e. $\alpha(t)=\alpha(0) e^{-\gamma\left(t-t_{0}\right)}$. In other words, the most probable value of $\alpha(t)$ coincides with the conditional average $\langle\alpha(t)| \alpha\left(t_{0}\right)>$.

Analogously, the most probable path starting from $\alpha_{0}$ and ending in $\alpha_{t}$ is given by the minimum of $\int_{t_{0}}^{t} d t^{\prime} \mathcal{L}\left[\alpha\left(t^{\prime}\right), \dot{\alpha}\left(t^{\prime}\right)\right]$ with the constraints $\alpha\left(t_{0}\right)=\alpha_{0}$ and $\alpha(t)=\alpha_{t}$. The minimum condition corresponds to

$$
\left[\int_{t_{0}}^{t}(\phi+\psi) d t^{\prime}\right]_{\min }=2\left(S\left(\alpha_{t}\right)-S\left(\alpha_{0}\right)\right)
$$

The extension to $N \geq 2$, does not present particular difficulties. For the case described by (7.7), the symmetry of the matrix $\mathbf{C}$ allows us to work in the diagonal representation of the white noise $\tilde{\eta}_{k}$, i.e. to deal with $N$ independent Markov processes. Therefore it suffices to replace the $\mathcal{L}$ in (7.15) for $N=1$ with

$$
\mathcal{L}(\boldsymbol{\alpha}, \dot{\boldsymbol{\alpha}})=\frac{1}{4 c} \sum_{k, k^{\prime}}\left(\dot{\alpha}_{k}+\sum_{j} B_{k, j} \alpha_{j}\right) A_{k, k^{\prime}}\left(\dot{\alpha}_{k^{\prime}}+\sum_{j} B_{k^{\prime}, j} \alpha_{j}\right)
$$

where $\mathbf{A}=\mathbf{C}^{-1}$. In addition one has

$$
\mathcal{L}(\boldsymbol{\alpha}, \dot{\boldsymbol{\alpha}})=\frac{1}{2}\left(\phi(\boldsymbol{\alpha}, \dot{\boldsymbol{\alpha}})+\psi(\mathbf{X})-\frac{d S(\boldsymbol{\alpha})}{d t}\right)
$$

and all the previous results hold for $N>1$. 


\subsubsection{The non Gaussian case}

The generalization of the above results to the nonlinear case, i.e.

$$
\frac{d \alpha_{k}}{d t}=f_{k}(\boldsymbol{\alpha})+\sqrt{2 c} \eta_{k} \quad k=1, \ldots, N
$$

where $\{\eta\}$ is a Gaussian process with $\left\langle\eta_{k}\right\rangle=0$ and $\left\langle\eta_{k}(t) \eta_{k^{\prime}}\left(t^{\prime}\right)\right\rangle=\delta_{k, k^{\prime}} \delta\left(t-t^{\prime}\right)$, is rather simple, at least at a formal level. Instead of (7.22), one obtains

$$
\mathcal{L}(\boldsymbol{\alpha}, \dot{\boldsymbol{\alpha}})=\frac{1}{4 c} \sum_{k, k^{\prime}}\left(\dot{\alpha}_{k}-f_{k}\right) A_{k, k^{\prime}}\left(\dot{\alpha}_{k^{\prime}}-f_{k^{\prime}}\right)
$$

A simple way to derive this result is the following: repeat the argument to obtain (7.13) for small $\tau$, and perform the path integral. Let us sketch the basic idea, for the one variable case:

$$
\frac{d \alpha}{d t}=f(\alpha)+\sqrt{2 c} \eta
$$

Denote $\alpha_{n}=\alpha\left(t_{0}+n \tau\right)$ and write the above Langevin equation in its discrete time approximation:

$$
\alpha_{n}-\alpha_{n-1}=f\left(\alpha_{n}\right) \tau+\sqrt{2 c \tau} w_{n}
$$

where $\left\{w_{n}\right\}$ are Gaussian random variables with $\left\langle w_{n}\right\rangle=0$ and $\left\langle w_{n} w_{n^{\prime}}\right\rangle=\delta_{n, n^{\prime}}$. Since the pdf of $w_{n}$ is Gaussian, the generalization of (7.13) merely requires that $\ell$ in (7.13) be replaced by

$$
\ell\left(\alpha_{n}, \alpha_{n-1}\right)=\frac{1}{4 c}\left[\frac{\left(\alpha_{n}-\alpha_{n-1}\right)}{\tau}-f\left(\alpha_{n}\right)\right]^{2} .
$$

The above procedure is heuristic and the result is not completely exact. Nevertheless it yields the leading term for small values of $c$. We do not discuss here this delicate point, the interested reader can see $[269,270]$.

The theory here outlined, for homogeneous systems, can be reformulated for spatially extended systems, as illustrated in the next subsections.

\subsection{Large deviations in extended nonequilibrium systems}

Various attempts have been made to extend Onsager-Machlup theory [267] to the large fluctuations of physical systems in nonequilibrium steady states [271]. Among them, the work by Jona-Lasinio and collaborators [272,167], is particularly relevant to our discussion. Such approach generalizes those of Derrida et al. [273], and in addition it leads to an independent derivation of the FR. The 
theory of $[272,167]$ begins from the assumption that a hydrodynamic-like description of the system at hand is possible hence, physically, it can be applied as far from equilibrium as the validity of the local thermodynamic equilibrium allows 24

The theory leads to the conclusion that the nonequilibrium entropy functional, which generalizes the Onsager-Machlup entropy to extended systems, is a nonlocal functional of the thermodynamic variables, hence that correlations are present over macroscopic scales. To illustrate these facts, consider stochastic models of interacting particles, whose number is locally conserved, in contact with particles reservoirs. Assume that these systems admit the hydrodynamic description

$$
\partial_{t} \varrho=\nabla \cdot\left[\frac{1}{2} D(\varrho) \nabla \varrho\right] \equiv \mathcal{D}(\varrho), \quad \varrho=\varrho(\mathbf{x}, t)
$$

where $\varrho$ is the vector of macroscopic observables, $\mathbf{x}$ is the macroscopic space variable, $t$ is the macroscopic time, $D$ is the diffusion matrix. Equation (7.26) is analogous, in the spatially extended case, to Eq.(7.7) without noise.

The mathematical theory is necessarily developed for very idealized models, such as the simple exclusion or the zero range stochastic processes 25 Nevertheless, the assumptions under which the theory holds are thought to be valid much more generally than in these cases, and are the following:

Assumptions: 1) The mesoscopic evolution is given by a Markov process $X_{t}$, which represents the configuration of the system at time $t$. The nonequilibrium steady state is described by a probability measure Prob over the trajectories of $X_{t}$;

2) the macroscopic description is given in terms of fields @ which constitute the local thermodynamic variables, whose evolution is described by (7.26), which has a unique stationary solution $\varrho$, under the given nonequilibrium boundary conditions;

3) Denoting by $I$ the time inversion operator defined by $I X_{t}=X_{-t}$, the probability measure Prob*, describing the evolution of the time reversed process $X_{t}^{*}$, and Prob are related by

$$
\operatorname{Prob}^{*}\left(X_{t}^{*}=\phi_{t}, t \in\left[t_{1}, t_{2}\right]\right)=\operatorname{Prob}\left(X_{t}=\phi_{-t}, t \in\left[-t_{2},-t_{1}\right]\right) .
$$

\footnotetext{
$\overline{24}$ This, of course, includes a very wide range of phenomena, well beyond the linear regime, which has quite wide applicability by itself.

25 These models are usually one-dimensional. Their hydrodynamic limit consists of a scaling of the microscopic space and time variables, $\tau$ and $\mathbf{r}$, with the macroscopic space and time variables given by $t=\tau / N^{2}$ and $\mathbf{x}=\mathbf{r} / N$, and the number of particles per unit length $N$ tending to infinity.
} 
Moreover, if $L$ is the generator of $X_{t}$, the adjoint dynamics is generated by the adjoint (with respect to the invariant measure $\mu$ ) operator $L^{*}$, which admits the adjoint hydrodynamic description

$$
\partial_{t} \varrho=\mathcal{D}^{*}(\varrho),
$$

4) The measure Prob admits a large deviation principle describing the fluctuations of $\varrho$, i.e. the probability for a large number $N$ of particles that the evolution of the random variable $\varrho_{N}$ deviates from the solution of (7.26), to follow a given path $\hat{\varrho}(t)$, in the interval $\left[t_{i}, t_{f}\right]$, goes like

$$
\operatorname{Prob}\left(\varrho_{N}\left(X_{N^{2} t}\right) \sim \hat{\varrho}(t),\left[t_{i}, t_{f}\right]\right) \approx e^{-N^{d}\left[\mathcal{S}\left(\hat{\varrho}\left(t_{i}\right)+J_{\left[t_{i}, t_{f}\right]}(\hat{\varrho})\right]\right.}
$$

where $d$ is the spatial dimension, $\mathcal{S}\left(\hat{\varrho}\left(t_{i}\right)\right)$ is the entropy cost to produce $\hat{\varrho}\left(t_{i}\right)$, and $J$ is the extra cost required to follow the given path (taking $\mathcal{S}(\tilde{\varrho})=0, J$ vanishes at $\varrho=\tilde{\varrho}$ ).

This machinery leads to the following results:

- The Onsager-Machlup theory is generalized by the introduction of the "adjoint hydrodynamic" equation (7.28), for the spontaneous fluctuations around nonequilibrium steady states. Then, assuming that $\mathcal{D}$ can be decomposed as

$$
\mathcal{D}(\varrho)=\frac{1}{2} \nabla \cdot\left(\chi(\varrho) \nabla \frac{\delta \mathcal{S}}{\delta \varrho}\right)+\mathcal{A}
$$

where $\mathcal{A}$ is a vector field orthogonal to the thermodynamic force $\delta \mathcal{S} / \delta \varrho$ (the functional derivative of the entropy with respect to the state), a temporal asymmetry arises in the fluctuation-relaxation paths. Indeed, a spontaneous fluctuation out of a nonequilibrium steady state follows a trajectory which is the time reversal of the relaxation path, according to the adjoint hydrodynamics, i.e. it solves

$$
\partial_{t} \varrho=-\mathcal{D}^{*}(\varrho)=-\mathcal{D}(\varrho)+2 \mathcal{A},
$$

which is not merely the time reversal of the hydrodynamic equation. Being orthogonal to the thermodynamic force, the term that breaks the time symmetry, $\mathcal{A}$, is not the one that contributes to the entropy production. Note that Eq. (7.28) describes the adjoint hydrodynamics, while Eq. (7.31) refers to the evolution of spontaneous fluctuations.

- A Hamilton-Jacobi type equation is given for the macroscopic entropy $\mathcal{S}(\rho)$. Expressing $J$ as

$$
J_{\left[t_{i}, t_{f}\right]}(\hat{\varrho})=\int_{t_{i}}^{t_{f}} d t \mathcal{L}\left(\varrho(t), \partial_{t} \hat{\varrho}(t)\right)
$$


and introducing the Hamiltonian as the Legendre transform of $\mathcal{L}\left(\varrho, \partial_{t} \varrho\right)$,

$$
\mathcal{H}(\varrho, Y)=\sup _{\xi}\left\{\int \xi Y d \mathbf{x}-\mathcal{L}(\varrho, \xi)\right\}
$$

the Hamilton-Jacobi type equation associated with the relation between $\mathcal{S}$ and $J$ is

$$
\mathcal{H}\left(\varrho, \frac{\delta \mathcal{S}}{\delta \varrho}\right)=0
$$

where $\mathcal{S}(\varrho)=\inf _{\hat{\varrho}} J_{[-\infty, 0]}(\hat{\varrho})$ is computed over all trajectories $\hat{\varrho}(t)$ connecting $\tilde{\varrho}$ to $\varrho$. Then, the steady state satisfies $\mathcal{H}(\tilde{\varrho}, 0)=0$.

- An H-theorem is given for $\mathcal{S}$ :

$$
\frac{d}{d t} \mathcal{S} \leq 0
$$

- A nonequilibrium fluctuation dissipation relation holds:

$$
\mathcal{D}(\varrho)+\mathcal{D}^{*}(\varrho)=\nabla \cdot\left(\chi(\varrho) \nabla \frac{\delta \mathcal{S}}{\delta \varrho}\right)
$$

These results have been later generalized to the case of systems subjected to external bulk forces, like electric fields [167].

\subsubsection{Temporal asymmetries in deterministic systems}

The question arises as to which aspects of the large deviation theory described above may be verified experimentally, or numerically tested in models other than the lattice gases for which it has been rigorously established. For instance, one usually assumes that the stochastic description is a reduced representation of some microscopic reversible dynamics, and should be recovered from that in some limit. However, various difficulties are to be faced, in order to answer that question. For instance, the dynamics of the Lorentz gas are proven equivalent to Markov processes, obtained by coarse graining with Markov partitions the relevant phase space [274]. On the other hand, the kind of stochastic process that one obtains depends on the particular form of graining, and concerns the phase space instead of the real space. Furthermore, it is not always possible to point out without ambiguities which quantities of the stochastic systems correspond to given quantities of the deterministic systems. Therefore, the identification of the deterministic dynamics which are consistent with a given stochastic process or, conversely, the identification of the stochastic processes which preserve the observable properties of given microscopic dynamics, is a rather difficult task, in general.

This, on the one hand, indicates that a direct comparison between the predictions of Refs. $[272,167,166]$ and the fluctuations of deterministic particle systems may be rather problematic, if not impossible, although desired [166]. At 
the same time, the behaviour of a macroscopic system cannot depend on such subtle and subjective issues like the form of the partitioning of the phase space; thus a test on deterministic systems of some aspect of the stochastic theory should be possible, in principle at least. Therefore, in Refs. [275,276,277] the prediction of the temporal asymmetries of fluctuations is tested, because the differences between hydrodynamics and adjoint hydrodynamics are largely responsible for the other theoretical predictions, and because such asymmetries look amenable to direct test. It would be interesting to test, on deterministic systems, other features of the stochastic theory.

The origin of the temporal asymmetry can be easily understood as follows. Consider the macroscopic deterministic dynamics described by

$$
\frac{d}{d t} \varrho=\mathcal{D}(\varrho)
$$

on $\mathcal{M} \subset \mathbb{R}^{n}$, where $\mathcal{D}$ is a vector field with a globally attracting fixed point $\varrho \in \mathcal{M}$. The $n$ components of $\varrho$ may represent the values taken by a scalar thermodynamic observable on the $n$ different sites of a spatially discrete system. Let the local mesoscopic dynamics be a perturbation of Eq.(7.36), with a Gaussian noise of covariance $\left\langle\xi_{i}(t) \xi_{j}\left(t^{\prime}\right)\right\rangle=K_{i j} \delta\left(t-t^{\prime}\right)$ and mean $\langle\xi(t)\rangle=0$, where $\mathbf{K}$ is a symmetric, positive definite matrix 26

$$
\frac{d}{d t} \varrho=\mathcal{D}(\varrho)+\xi
$$

This allows different evolutions between one initial state $\varrho_{i}=\varrho\left(t_{i}\right)$ and one later state $\varrho_{f}=\varrho\left(t_{f}\right)$. The different paths connecting $\varrho_{i}$ to $\varrho_{f}$ occur with different probabilities and Eq.(7.36) can be obtained from the maximization of probabilities of the form $P \propto \exp \left(-F_{\left[t_{i}, t_{f}\right]}(\varrho)\right)$, which depend on $\varrho_{i}, \varrho_{f}$ and on the path connecting them:

$$
F_{\left[t_{i}, t_{f}\right]}(\varrho)=\frac{1}{2} \int_{t_{i}}^{t_{f}}\left\langle\frac{d}{d t} \varrho-\mathcal{D}, \frac{d}{d t} \varrho-\mathcal{D}\right\rangle \mathrm{d} t
$$

where the scalar product is defined by $\langle x, y\rangle=x^{T} \mathbf{K}^{-1} y$, and the superscript $T$ indicates transposition. Therefore, Eq.(7.37) is analogous to Eq.(7.7), and Eq.(7.38) corresponds to Eq.(7.22), with $\mathbf{K}^{-1}$ in place of $\mathbf{A} / 2 c$. Now, decompose the vector field $\mathcal{D}$ as

$$
\mathcal{D}(\varrho)=-\frac{1}{2} \mathbf{K} \nabla_{\varrho} V(\varrho)+\mathcal{A}(\varrho), \quad \text { with } \quad\left\langle\mathbf{K} \nabla_{\varrho} V, \mathcal{A}\right\rangle=0
$$

where $\nabla_{\varrho}$ indicates differentiation with respect to the components of $\varrho$, and let $\tilde{\varrho}$ be a minimum of $V$, with $V(\tilde{\varrho})=0$, separating dissipative contributions

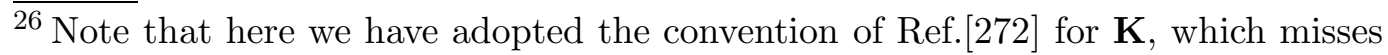
a factor 2 with respect to more standard notation used in Section 7.1. 
to $\mathcal{D}$ from non-dissipative ones, as common in diffusion processes described by finite dimensional Langevin equations [270]. Substituting (7.39) in (7.38), one obtains

$$
2 F_{\left[t_{i}, t_{f}\right]}(\varrho)=\int_{t_{i}}^{t_{f}}\left\langle\frac{d}{d t} \varrho+\frac{1}{2} \mathbf{K} \nabla_{\varrho} V-\mathcal{A}, \frac{d}{d t} \varrho+\frac{1}{2} \mathbf{K} \nabla_{\varrho} V-\mathcal{A}\right\rangle \mathrm{d} t
$$

which is minimized by the relaxational path converging to $\tilde{\varrho}$ and obeying

$$
\frac{d}{d t} \varrho=-\frac{1}{2} \mathbf{K} \nabla_{\varrho} V(\varrho)+\mathcal{A}(\varrho)
$$

i.e. obeying (7.36). However, the integrand of (7.40) is a quadratic form and has a second minimizing path, which is revealed by an integration by parts. This yields:

$$
\begin{aligned}
2 F_{\left[t_{i}, t_{f}\right]}(\varrho)= & \int_{t_{i}}^{t_{f}}\left\langle\frac{d}{d t} \varrho-\frac{1}{2} \mathbf{K} \nabla_{\varrho} V-\mathcal{A}, \frac{d}{d t} \varrho-\frac{1}{2} \mathbf{K} \nabla_{\varrho} V-\mathcal{A}\right\rangle \mathrm{d} t+ \\
& +2\left[V\left(\varrho_{f}\right)-V\left(\varrho_{i}\right)\right]
\end{aligned}
$$

whose second term has no variation, once the initial and final states are fixed. Therefore, the second possible kind of evolution for $\varrho$ corresponds to the fluctuations away from $\tilde{\varrho}$, which obey

$$
\frac{d}{d t} \varrho=-\mathcal{D}^{*}(\varrho)=\frac{1}{2} \mathbf{K} \nabla_{\varrho} V+\mathcal{A}(\varrho)=-\mathcal{D}+2 \mathcal{A}
$$

The qualitative properties of the deterministic dynamics do not depend on $\mathcal{A}$, as the time derivative of the Lyapunov function $V$ does not depend on $\mathcal{A}$. Curiously, the asymmetry of the fluctuation paths depends only on this non dissipative term.

In the hydrodynamic limit, $\mathcal{D}$ becomes the elliptic differential operator of Eqs.(7.26,7.30), where $\mathbf{K}$ turns into $\nabla \cdot \chi(\varrho) \nabla, \nabla_{\varrho}$ into the functional derivative $\delta / \delta \varrho$ and $V(\varrho)$ into $\mathcal{S}(\varrho)$. In the theory developed by Onsager and Machlup, $\mathcal{A}=0$ because only the small fluctuations were considered, and the linear response regime was assumed.

The above suggests that sufficiently chaotic systems of interacting particles should typically have asymmetric fluctuation paths. This has been indeed confirmed in $[276,277]$ for the nonequilibrium FPU model, as well as for the shearing system of Eq.(5.2). In Ref.[276] it was then explained that the current fluctuations of the many particles nonequilibrium Lorentz gas, studied in [275], had to be symmetric, as observed, because of the lack of interactions among those particles. It remains an open question to see which kinds of interactions lead to symmetric and which to asymmetric nonequilibrium fluctuation paths. 
To properly deal with deterministic systems of finitely many particles, Refs.[275,276,277] introduced various notions of fluctuation-relaxation paths, together with various criteria to assess their asymmetry. In the following subsection, we consider only one of these notions, for the local heat flux of the nonequilibrium FPU model.

\subsubsection{Heat flux fluctuation-relaxation paths in the FPU model}

An observable $X: \mathcal{M} \rightarrow I R$, for a system made of a finite number of particles, looks very noisy, in general. Therefore, given an initial condition $\Gamma$ in the steady state, we search for the most likely path that starts at $X_{t_{i}}=\bar{X}$, reaches a certain fluctuation value $\mathcal{T}(X)$ at time $\hat{t}$, and later returns to $\bar{X}=X_{t_{f}}$. By path we mean the trajectory segment $\left\{X_{\hat{t}+\tau}, \tau \in\left[-\tau_{0}, \tau_{0}\right]\right\}$ where, for simplicity, $X_{t}$ denotes $X\left(S^{t} \Gamma\right)$, and $\bar{X}$ is the corresponding time average of $X$. The time $\tau_{0}$ is a positive constant, that needs to be identified case by case, and represents the typical fluctuation time. This notion may look odd, since a part of a given fluctuation-relaxation path is sampled more than once (typically twice), but is appropriate to study the symmetry properties of paths, because it does not alter their symmetry, and does not neglect any of them. Furthermore, the results obtained so far are robust against variations of this definition $[277,276]$.

Given $n$ paths, they can be arranged to produce a two dimensional histogram, by partitioning the rectangle $\left[-\tau_{0}, \tau_{0}\right] \times\left[\min _{\tau, s} X_{\tau}^{(s)}, \max _{\tau, s} X_{\tau}^{(s)}\right]$ with rectangular bins, and evaluating the frequency of visitation of each bin. The "crest" of the histogram represents the most likely path in the sample, the one which should become the unique (deterministic) path in the large system limit. In [276], a number $M$ of tests is performed, each producing a different histogram and a different crest, so that the maximum, minimum and average crests can be defined as follows. Let $t \in\left[-\tau_{0}, \tau_{0}\right]$ and $j=1, \ldots, M$ be the index of the crest, then, denoting by $\mathcal{C}^{j}$ the crest produced in the $j$-th test,

$$
\mathcal{C}_{a v}(t)=\frac{1}{M} \sum_{j=1}^{M} \mathcal{C}^{j}(t)
$$

is the average crest;

$$
\underline{\mathcal{C}}(t)=\min _{j=1, \ldots, M} \mathcal{C}^{j}(t)
$$

is the minimum crest, and

$$
\overline{\mathcal{C}}(t)=\max _{j=1, \ldots, M} \mathcal{C}^{j}(t)
$$

is the maximum crest.

To assess the symmetry properties of a crest $\mathcal{C}$, discretize the time interval 


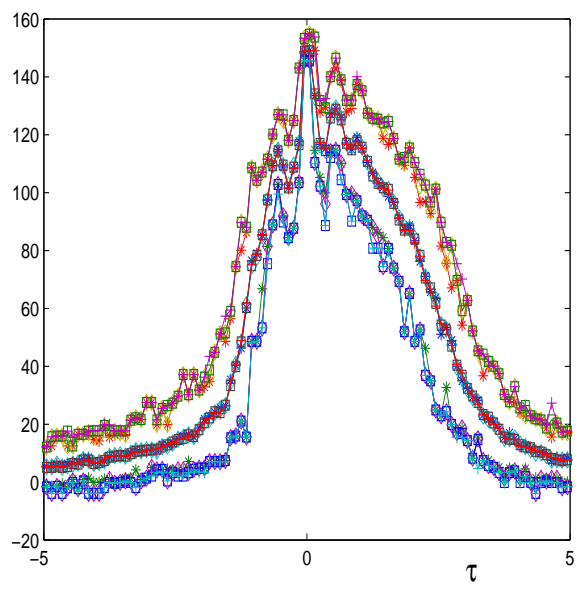

Fig. 21. Crest of the heat flux $f$ for an FPU chain with $N=66, T_{\ell}=70, T_{r}=20$, $\bar{f} \simeq 16.2$, and $\mathcal{T} \simeq 148$. The highest line represents $\overline{\mathcal{C}}$, the intermediate line represents $\mathcal{C}_{a v}$, and the lowest line represents $\underline{\mathcal{C}}$. Each line is the overlap of the four crests obtained with $M=39,59,79,99$.

$\left[0, \tau_{0}\right]$ in $b_{\tau}$ sub-intervals, and introduce the asymmetry coefficient as

$$
\alpha_{c}(\mathcal{C})=\frac{2}{b_{\tau}(\mathcal{T}-\bar{X})}\left(\sum_{p=\frac{b_{\tau}}{2}+1}^{b_{\tau}} \mathcal{C}(p)-\sum_{p=1}^{\frac{b_{\tau}}{2}} \mathcal{C}(p)\right)
$$

where, the normalizing factors are introduced only for convenience. A crest is called symmetric if its asymmetry coefficient vanishes. The asymmetry coefficients can take positive as well as negative values, and random oscillations of a fluctuation path around its symmetric relaxation image result in a vanishing asymmetry coefficient. With these definitions, and $M=20$, the heat flux in a central portion of the chain, of the nonequilibrium FPU model, with $N=150$ oscillators, produces results like

$$
\alpha_{c}(\underline{\mathcal{C}})=0.170, \quad \alpha_{c}\left(\mathcal{C}_{a v}\right)=0.194, \quad \alpha_{c}(\overline{\mathcal{C}})=0.211
$$

for a large temperature difference between the ends of the chain $\left(T_{\ell}=200\right.$ and $T_{r}=20$ ), and for a fluctuation value $\mathcal{T}$ three standard deviations higher than the mean. Here, the gap between the maximum and minimum crests asymmetries can be taken as the numerical uncertainty on the asymmetry of the crest. The result is that temporally asymmetric fluctuation-relaxation paths are found in time reversal invariant dynamics. Figure 21 illustrates these facts for a chain with fewer oscillators, for which a relatively large number of tests can be performed. In this case, it is interesting to see that the three kinds of crests do not appreciably change when the number of tests grows over $M=20$, which is an indication of the accuracy achieved in the construction of the crests. In Ref.[278], a justification of the ubiquity of the temporally asymmetric fluctuation-relaxation paths, in nonequilibrium particle systems, 
is given in terms of correlation functions.

\subsection{The additivity principle}

The theory developed in Refs.[272,167] has a non-local nature, as easily understood in the works of Derrida and collaborators [273,166], who independently worked out the explicit solutions of what can now be seen as specific examples of the theory of $[272,167]$. Actually, explicitly computing the large deviation functional of the current in systems like the symmetric and asymmetric simple exclusion processes, Bodineau and Derrida realized that a certain additivity principle describes the corresponding steady states.

The idea is the following: let a one dimensional system have length $L+L^{\prime}$ and be in contact with two reservoirs of particles at densities $\varrho_{a}$ and $\varrho_{b}$. Express the probability of the current integrated in a time $t, Q_{t}=j t$ say, as

$$
\operatorname{Prob}_{L+L^{\prime}}\left(j, \varrho_{a}, \varrho_{b}\right) \sim e^{-t F_{L+L^{\prime}}\left(j, \varrho_{a}, \varrho_{b}\right)} .
$$

Then, the additivity principle relates $F_{L+L^{\prime}}$ to the large deviation functionals of the subsystems of sizes $L$ and $L^{\prime}$, by taking

$$
\operatorname{Prob}_{L+L^{\prime}}\left(j, \varrho_{a}, \varrho_{b}\right) \sim \max _{\varrho}\left\{\operatorname{Prob}_{L}\left(j, \varrho_{a}, \varrho\right) \operatorname{Prob}_{L^{\prime}}\left(j, \varrho, \varrho_{b}\right)\right\}
$$

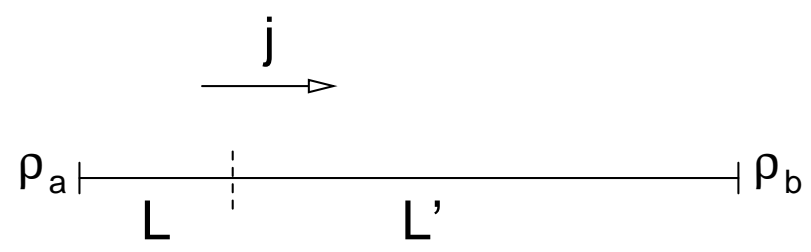

Fig. 22. Sketch of a one-dimensional system between two reservoirs $\rho_{a}$ and $\rho_{b}$, which is virtually divided into two sub-systems of length $L$ and $L^{\prime}$ respectively. An average current $j$ flows through the system (assuming for instance that $\rho_{a}>\rho_{b}$ ).

In other words, one assumes that the probability of transporting $j$ between the reservoirs at densities $\varrho_{a}$ and $\varrho_{b}$, is the maximum over $\varrho$ of the probabilities of transporting the same currents between two reservoirs at densities $\varrho_{a}$ and $\varrho$, times the probabilities of transporting it between two reservoirs at densities $\varrho$ and $\varrho_{b}$. Then, in the large system limit, in which boundary contributions become negligible, the relevant large deviation functional takes the form

$$
F_{L+L^{\prime}}\left(j, \varrho_{a}, \varrho_{b}\right)=\max _{\varrho}\left\{F_{L}\left(j, \varrho_{a}, \varrho\right)+F_{L^{\prime}}\left(j, \varrho, \varrho_{b}\right)\right\}
$$

In the case of nonequilibrium boundary conditions (i.e. $\varrho_{a} \neq \varrho_{b}$ ), this results in a non-local relation for the steady state density profile, as shown in the next 
subsection for an exactly solvable model. Introducing the scaling hypothesis

$$
F_{L}\left(j, \varrho_{a}, \varrho_{b}\right) \simeq \frac{1}{L} G\left(L j, \varrho_{a}, \varrho_{b}\right)
$$

and observing that the optimal profile connecting $\varrho_{a}$ to $\varrho_{b}$ is the same for both $j$ and $-j$, in the steady state, Bodineau and Derrida obtain also the validity of the FR for the current $j$, in the form

$$
G\left(-j, \varrho_{a}, \varrho_{b}\right)=G\left(j, \varrho_{a}, \varrho_{b}\right)-2 j \int_{\varrho_{b}}^{\varrho_{a}} \frac{D(\varrho)}{\sigma(\varrho)} d \varrho
$$

where $D(\varrho)$ and $\sigma(\varrho)$, in the large $L$ limit, are defined as follows: let $\varrho_{a}=\varrho+\Delta \varrho$ and $\varrho_{b}=\varrho$, with small $\Delta \varrho$, then $\left\langle Q_{t}\right\rangle / t=D(\varrho) \Delta \varrho / L$ and $\left\langle Q_{t}^{2}\right\rangle / t=\sigma(\varrho) / L$.

\subsubsection{The symmetric simple exclusion process}

The symmetric simple exclusion process affords one of the few nonequilibrium systems for which the large deviation function of the density can be computed in an explicit way. This model consists of a 1-dimensional lattice with $L$ sites, which can be either empty or occupied by one particle. At each time step, of length $\Delta t$ every particle can jump with equal probability $\Delta t$ to the right, if the site on the right is empty, or to the left, if the site on the left is empty. At the left boundary of the lattice (site 1), particles are injected with probability $\alpha \Delta t$, if 1 is empty, and are removed with probability $\gamma \Delta t$, if 1 is occupied. Similarly, at the right boundary (site L), particles are injected with probability $\delta \Delta t$ and removed with probability $\beta \Delta t$. This is equivalent to have a reservoir of particles at density $\varrho_{a}=\alpha /(\alpha+\gamma)$ on the left, and one of density $\varrho_{b}=\delta /(\delta+\beta)$ on the right of the lattice. Therefore, if $\tau_{i}=0,1$ is the random variable equal to 0 when site $i$ is empty, and equal to 1 when it is occupied, one obtains

$$
\begin{aligned}
& \frac{d\left\langle\tau_{1}\right\rangle}{d t}=\alpha-(\alpha+\gamma+1)\left\langle\tau_{1}\right\rangle+\left\langle\tau_{2}\right\rangle \\
& \frac{d\left\langle\tau_{i}\right\rangle}{d t}=\left\langle\tau_{i-1}\right\rangle-2\left\langle\tau_{i}\right\rangle+\left\langle\tau_{i+1}\right\rangle, \quad 2 \leq i \leq L-1 \\
& \frac{d\left\langle\tau_{L}\right\rangle}{d t}=\left\langle\tau_{L-1}\right\rangle-(\delta+\beta+1)\left\langle\tau_{L}\right\rangle+\delta .
\end{aligned}
$$

The steady state, which corresponds to vanishing derivatives, takes the form

$$
\left\langle\tau_{i}\right\rangle=\frac{\varrho_{a}\left(L+\frac{1}{\beta+\delta}-i\right)+\varrho_{a}\left(\frac{1}{\alpha+\gamma}+i-1\right)}{L+\frac{1}{\alpha+\gamma}+\frac{1}{\beta+\delta}-1}
$$

and the current takes the form

$$
J=\left\langle\tau_{i}-\tau_{i+1}\right\rangle=\frac{\varrho_{a}-\varrho_{b}}{L+\frac{\varrho_{a}}{\alpha}+\frac{\varrho_{b}}{\delta}-1}
$$


which is proportional to the gradient $\left(\varrho_{a}-\varrho_{b}\right) / L$, when $L \gg 1, \varrho_{a} / \alpha, \varrho_{b} / \delta$. At equilibrium, with $\varrho_{a}=\varrho_{b}=\hat{\varrho}$, and in the large $L$ limit, if the lattice has unit length, the large deviation functional takes the form

$$
F(\varrho)=\int_{0}^{1} B(\varrho(x), \varrho) \mathrm{d} x, \quad \text { with } \quad B(\varrho, \varrho)=(1-\varrho) \log \frac{1-\varrho}{1-\varrho}+\varrho \log \frac{\varrho}{\varrho} .
$$

The functional is thus a local function of $\varrho(x)$, and is convex. Let us note that $B(\varrho, \hat{\varrho})$ is nothing but the Creamer's function corresponding to the binomial distribution, see Appendix B. Differently, in the nonequilibrium case, $\varrho_{a} \neq \varrho_{b}$, one has

$$
F(\varrho)=\int_{0}^{1}\left[B(\varrho(x), f(x))+\log \frac{f^{\prime}(x)}{\varrho_{b}-\varrho_{a}}\right]
$$

where $f$ is the monotone solution of

$$
\varrho=f+\frac{f(1-f) f^{\prime \prime}}{f^{\prime 2}}, \quad f(0)=\varrho_{a}, \quad f(1)=\varrho_{b} .
$$

This result, which was first obtained in [279] and, later, as a special case of the theory of [272], shows that the value of $f$ at position $x$ depends on the profile $\varrho(y)$ at all points $y$, hence that $F$ is non-local.

\section{Conclusions}

In this report we have summarized the history and the state of the art of the studies concerning the response of physical systems to external actions. This has evidenced how the statistical mechanics community has gradually shifted its focus from the investigation of equilibrium and near equilibrium phenomena, to strongly nonequilibrium phenomena, which cannot be understood in terms of thermodynamic quantities. Similarly, the interest has moved from the construction of a microscopic interpretation of the macroscopic phenomena, for which the thermodynamic limit of finite systems is necessary, to the study of the properties of mesoscopic and microscopic objects, which can be understood as systems of finitely many microscopic components. These objects are presently under intense investigation, because they appear to be extremely interesting from both fundamental and applied viewpoints. From the applied viewpoint, it is important to understand their behaviour, because of the emerging nanotechnology, which impacts also on our ability to manipulate biological systems. From the fundamental point of view, it is now obvious that they must be described by phenomenological equations which differ from those of the macroscopic world. But the classification of the phenomena which are possible at the mesoscopic and microscopic level is still to be done, and a consistent theory of such phenomena is still lacking. 
Nevertheless, the body of knowledge acquired in the past century, in the construction of the microscopic theory of the response of macroscopic systems, appears to be an ideal springboard to decisively leap into the largely unexplored realm of strongly nonequilibrium systems, and of microscopic objects. Indeed, this knowledge has been based on the characterization of the fluctuations which, although macroscopically not observable, are evident if the thermodynamic limit is not taken. This is why we believe that a review of the response theory stretching up to present times, with emphasis on nonstandard issues (granular media, fluids, nano-systems and biological systems), was needed and should be useful in advancing our understanding of the physical world.

\section{Acknowledgments}

We thank our colleagues with whom we collaborated on the issues here discussed: A. Baldassarri, A. Barrat, G. Benettin, L. Biferale, G. Boffetta, F. Cecconi, M. Cencini, E.G.D. Cohen, I. Daumont, D.J. Evans, M. Falcioni, G. Gallavotti, A. Gamba, C. Giberti, S. Isola, O.G. Jepps, G. Lacorata, S. Lepri, V. Loreto, J. Lloyd, C. Mejia-Monasterio, G. Morriss, S. Musacchio, M. Niemeyer, C. Paneni, D.J. Searles, E. Segre, T. Tél, E. Trizac, F. van Wijland, C. Vernia, P. Visco and J. Vollmer.

In addition we are grateful to K. Kaneko, M.H. Lee and F. Ritort for correspondence and useful remarks.

\section{A Appendix: Models of granular gases}

The most common model of fluidized granular matter is the gas of inelastic hard spheres in $d$ dimensions (reviews of recent results can be found in [280] and [281]), interacting through binary inelastic collisions. Initially the $N$ particles are placed in a box of volume $V=L^{d}$, at random positions and with random velocities, typically taken from a Gaussian distribution. Since we discuss monodisperse as well as bidisperse systems, we denote by $s_{i}, \sigma_{i}$ and $m_{i}$ respectively the species, the diameter and the mass of particle $i$. The use of $\sigma$ and $m$ without indexes will denote the monodisperse case. When particles $i$ and $j$ collide, the instantaneous change of their velocities is given by

$$
\mathbf{v}_{i}^{\prime}=\mathbf{v}_{i}-\left(1+r_{i j}\right) \frac{m_{j}}{m_{i}+m_{j}}\left[\left(\mathbf{v}_{i}-\mathbf{v}_{j}\right) \cdot \hat{\sigma}_{i j}\right] \hat{\sigma}_{i j}
$$

where $r_{i j} \in[0,1]$ is the restitution coefficient, $m_{i}$ and $m_{j}$ are the masses of the particles and $\hat{\sigma}_{i j}$ is the unit vector joining the centers of the particles. When 
$r_{i j}=1$ the gas is elastic and no energy is dissipated in the collisions. In all other cases the collisions are dissipative. Different kinds of experimental setups can be reproduced by this model, provided that a suitable driving mechanism is added. A widely accepted model for the investigation of strongly fluidized granular gases, reduces the effect of the interaction between the grains and the fluidizing agent (e.g. an air flow or random collisions with the vibrating plate) to that of a "thermal" bath coupled to every particle. Between collisions, the particles are subjected to a random force given by white noise, with the possible addition of a viscous term. The equation of motion for a particle is then

$$
m_{i} \frac{d \mathbf{v}_{i}}{d t}=\mathbf{F}_{i}^{c o l}+\boldsymbol{\eta}_{i}-\gamma_{s_{i}} \mathbf{v}_{i}
$$

where $\mathbf{F}_{i}^{c o l}$ is the force due to inelastic collisions, $\gamma_{s_{i}}$ is the viscosity coefficient of species $s_{i}$ and $\boldsymbol{\eta}_{i}$ is the Gaussian stochastic force, with $\left\langle\eta_{i \alpha}(t) \eta_{j \beta}\left(t^{\prime}\right)\right\rangle=$ $\Gamma_{s_{i}}^{2} \delta_{i j} \delta_{\alpha \beta} \delta\left(t-t^{\prime}\right)$, where the Greek indices refer to Cartesian coordinates. The granular temperature of species $s$ is given by its mean kinetic energy $T_{g}^{s}=$ $m_{s}\left\langle v^{2}\right\rangle_{s} / d$ where \langle\rangle$_{s}$ is an average restricted only to particles of species $s$. This definition is important in view of the lack of energy equipartition observed in granular mixtures.

From the generic equation of motion (A.2), many different models can be obtained. When $\gamma_{1}=\gamma_{2}=\gamma, \Gamma_{1}=\Gamma_{2}=\Gamma, r_{11}=r_{12}=r_{22}=r$, and all the particles are identical, the gas is monodisperse. The "cooling granular gas" is recovered when $\gamma=\Gamma=0$ [113]. In such a case the particles follow ballistic trajectories until they collide and lose energy. The kinetic energy of the particles in their center of mass frame decreases with time, justifying the term "cooling". The homogeneous cooling is an idealized situation obtained when the positions of the particles are disregarded [113]. This situation is considered the analogous of the perfect rarefied gas in the realm of granular matter. For this model an equation for the evolution of the single particle velocity pdf $f(\mathbf{v}, t)$ can be written, in the form [282]

$$
\left(\partial_{t}+\mathbf{v} \cdot \nabla\right) f(\mathbf{v}, t)=J[f, f](\mathbf{v}, t),
$$

where, in the Boltzmann-Grad limit, the collision operator $J$, for hard spheres, takes the form:

$J[f, f]\left(\mathbf{v}_{1}, t\right)=\frac{1}{\ell} \int_{\mathbf{v}_{12} \cdot \boldsymbol{\sigma}>0} \mathrm{~d} \mathbf{v}_{2} \mathrm{~d} \boldsymbol{\sigma}\left(\mathbf{v}_{12} \cdot \boldsymbol{\sigma}\right)\left(\frac{1}{r^{2}} f\left(\mathbf{v}_{1}^{*}, t\right) f\left(\mathbf{v}_{2}^{*}, t\right)-f\left(\mathbf{v}_{1}, t\right) f\left(\mathbf{v}_{2}, t\right)\right)$.

In this last expression, the relative velocity $\mathbf{v}_{12}=\mathbf{v}_{1}-\mathbf{v}_{2}$ has been used, as well as the pre-collisional velocities $\mathbf{v}_{1}^{*}, \mathbf{v}_{2}^{*}$ which can be obtained from $\mathbf{v}_{1}, \mathbf{v}_{2}$ inverting the collision rule (A.1) for particles 1 and 2 .

When $\Gamma \neq 0$ the effect of external random forces balances the energy dissipated in collisions, and a statistically stationary state is reached. Two possibilities have been considered in the literature: 
- the heat bath with viscosity $[133,134,135,136]$, i.e. $\gamma \neq 0$ : in this case a "bath temperature" can be defined as $T_{b}=\Gamma^{2} / 2 \gamma$. This corresponds to the temperature of a gas obeying equation (A.2) with elastic collisions or without collisions. The same temperature can be observed if the viscosity is very high, i.e. when $\gamma \gg 1 / \tau_{c}$ where $\tau_{c}$ is the mean free time between collisions; when $\gamma \ll 1 / \tau_{c}$ and $r<1$ the gas still reaches a stationary regime, but its granular temperature is in general smaller than $T_{b}$ and, therefore, the system is out of equilibrium [283];

- the heat bath without viscosity $[137,138,139]$, i.e. $\gamma=0$; in this case to obtain the correct elastic limit one must reduce the driving intensity $\Gamma \sim$ $1-r^{2}$ as $r \rightarrow 1$, so that the average kinetic energy per particle and per degree of freedom $T_{g}$ does not diverge.

These two models show a rich range of phenomena, that are usually observed in driven granular gases. The statistical properties of the stationary state obtained by those models are different from those of an elastic gas in contact with a thermal bath: the most important difference is that the velocity distribution is non-Gaussian with enhanced high-energy tails [282]. The other typical feature is that the inelastic collisions reduce the relative velocity of particles and make re-collisions more likely: this is a source of correlations which emerges in the form of density clusters and velocity parallelization (vortices and other structures $[134,138,139])$. The hydrodynamic transport coefficients for these models have been calculated from the Chapman-Enskog approximated solution of the Boltzmann equation in [284], while an analytical study of the Einstein relation can be found in [119].

Other models can be derived from the previous ones. A useful approximation is the so-called Direct Simulation Monte Carlo, or DSMC [285,286,287], which consists in disregarding the relative position of particles in order to drastically reduce the probability of a re-collision: this is usually considered to be equivalent to the Molecular Chaos assumption in the $N \rightarrow \infty$ limit . Here, we consider the uniform gas. This model is nonetheless useful to test also spatial effects, see for example $[134,286]$. In this case the collisions occur with a probability which is proportional to the relative velocity of the particles, in order to be consistent with the kernel of the collision integral of the Boltzmann equation (A.4). A further simplification is operated in the so-called Inelastic Maxwell Models (see [124,125] and references therein). These models are gases of inelastic particles without positions, where collisions occur with a constant probability, i.e. not dependent upon the relative velocity. They are inspired by the so-called Maxwell Molecules, an elastic kinetic model having the same property due to the particular inter-particle (soft core) potential. Inelastic Maxwell Models have a very simple Boltzmann equation that can be analytically solved in the one dimensional case [124,125]. Other properties can be obtained in an analytical form, for example it is possible to demonstrate a general relation between the response $R(t)$ of a particle to the sudden pertur- 
bation of its velocity at time 0 , with a very large set of correlation functions, including the self-correlation $[117,118]$ :

$$
R(t)=C_{f}(t)=\frac{\langle v(t) f[v(0)]\rangle}{\langle v(0) f[v(0)]\rangle}=\exp \left(-\frac{r(r+1)}{4} t\right)
$$

with any generic function $f$ of the initial velocity value.

\section{B Appendix: Large Deviations in a nutshell}

For the sake of self-consistency, we briefly discuss the basic aspects of the Large Deviations theory. Roughly speaking, one can say that the Large Deviations theory is a generalization of the two most important limit theorems of probability theory, i.e. the law of large numbers, and the central limit theorem [288,289].

Consider a simple example: a sequence of independent tosses of an unfair coin. The possible outcomes are head $(+1)$ or tail $(-1)$. Denote the possible result of the $n$-th toss by $x_{n}$, where head has probability $\alpha$, and tail has probability $1-\alpha$. Let $Y_{N}$ be the mean value after $N$ tosses,

$$
Y_{N}=\frac{1}{N} \sum_{n=1}^{N} x_{n}
$$

If $N \gg 1$, a straightforward application of the central limit theorem gives

$$
\operatorname{Prob}\left(Y<Y_{N}<Y+d Y\right) \simeq P_{N}(Y) d Y \sim e^{-\frac{N(Y-\langle Y\rangle)^{2}}{2 \sigma^{2}}} d Y,
$$

where $\langle Y\rangle=2 \alpha-1, \sigma^{2}=4 \alpha(1-\alpha), P_{N}(Y)$ is the pdf of the random variable (B.1). Since $Y_{N}$ lies between -1 and 1, it is easy to realize that (B.2) is accurate only for small deviations of $Y_{N}$ from the mean (namely $\left|Y_{N}-\langle Y\rangle\right|<$ $O(1 / \sqrt{N}))$

A natural way to introduce the large deviations and show their deep relation with the concept of entropy is to perform a combinatorial computation. The number of ways in which $K$ heads occur in $N$ tosses is $N ! /[K !(N-K) !]$, therefore, the exact binomial distribution yields

$$
\operatorname{Prob}\left(Y_{N}=\frac{2 K}{N}-1\right)=\frac{N !}{K !(N-K) !} \alpha^{K}(1-\alpha)^{N-K} .
$$

Using Stirling's approximation and writing $K=p N$ and $N-K=(1-p) N$ one obtains

$$
P_{N}(Y=2 p-1) \sim e^{-N I(\alpha, p)},
$$

where

$$
I(\alpha, p)=p \ln \frac{p}{\alpha}+(1-p) \ln \frac{1-p}{1-\alpha} .
$$


Note that $I(\alpha, p)$ is called "relative entropy" (or Kullback-Leibler divergence), and $I(\alpha, p)=0$ for $\alpha=p$, while $I(\alpha, p)>0$ for $\alpha \neq p$. It is easy to repeat the argument for the multinomial case, where $x_{1}, \ldots, x_{N}$ are independent variables that take $m$ possible different values $a_{1}, a_{2}, \ldots, a_{m}$ with probabilities $\pi_{1}, \pi_{2}, \ldots, \pi_{m}$. In the limit $N \gg 1$, the probability of observing the frequencies $f_{1}, f_{2}, \ldots, f_{m}$ is

$$
\operatorname{Prob}_{N}\left(\left\{f_{j}\right\} \simeq\left\{p_{j}\right\}\right) \sim e^{-N I(\{p\},\{\pi\})}
$$

where

$$
I(\{p\},\{\pi\})=\sum_{j=1}^{m} p_{j} \ln \frac{p_{j}}{\pi_{j}},
$$

is called "relative entropy" of the probability $\{p\}$, with respect to the probability $\{\pi\}$. Such a quantity measures the discrepancy between $\{p\}$ and $\{\pi\}$ in the sense that $I(\{p\},\{\pi\})=0$ if and only if $\{p\}=\{\pi\}$, and $I(\{p\},\{\pi\})>0$ if $\{p\} \neq\{\pi\}$.

From the above computation one understands that it is possible to go beyond the central limit theory, and to estimate the statistical features of extreme (or tail) events, as the number of observations grows without bounds. Writing $I(p, \alpha)$ in terms of $Y=2 p-1$, Eq. (B.4) becomes

$$
P_{N}(Y) \sim e^{-N S(Y)},
$$

with

$$
S(Y)=\frac{1+Y}{2} \ln \frac{1+Y}{2 \alpha}+\frac{1-Y}{2} \ln \frac{1-Y}{2(1-\alpha)} .
$$

The function $S(Y)$ is called Cramer's function. Of course, for $p$ close to $\alpha$, i.e. $Y \simeq\langle Y\rangle$, a Taylor expansion reproduces the central limit theorem (B.2).

However Eq. (B.6) has a general validity, and can be derived in different ways, which show how the shape of $S(Y)$ is related to the behaviour of the moments of the variable $x$. In particular it is possible to see that $S(Y)$ can be expressed in terms of a Legendre transform:

$$
S(Y)=\sup _{q}[q Y-L(q)],
$$

where $L(q)$ is the "Cumulants Generating Function" given by

$$
L(q)=\ln \left\langle e^{q x}\right\rangle .
$$

Let us sketch the argument. Consider the quantities $\left\langle e^{q N Y_{N}}\right\rangle$ which can be written in two ways:

$$
\begin{aligned}
& \left\langle e^{q N Y_{N}}\right\rangle=\left\langle e^{q x}\right\rangle^{N}=e^{N L(q)} \\
& \left\langle e^{q N Y_{N}}\right\rangle=\int e^{q N Y_{N}} P_{N}\left(Y_{N}\right) d Y_{N} \sim \int e^{[q Y-S(Y)] N} d Y
\end{aligned}
$$


whose identification leads to

$$
\int e^{[q Y-S(Y)] N} d Y \sim e^{N L(q)}
$$

and, in the limit of large $N$, using the steepest descent method, one has

$$
L(q)=\sup _{Y}[q Y-S(Y)]
$$

which is the inverse of $((\mathrm{B} .7))$. Since it is possible to show that $S(Y)$ is convex, Equations (B.7) and (B.12) are fully equivalent.

For the more general and interesting case of dependent variables, $L(q)$ is defined as

$$
L(q)=\lim _{N \rightarrow \infty} \frac{1}{N} \ln \left\langle e^{q \sum_{n=1}^{N} x_{n}}\right\rangle,
$$

and (B.7) is exact if $S(Y)$ is convex, otherwise Eq. (B.7) gives the convex envelop of the correct $S(Y)$.

Let us note that the Cramer function must obey some constraints:

a) $S(Y)>0$ for $Y \neq\langle Y\rangle$;

b) $S(Y)=0$ for $Y=\langle Y\rangle$;

c) $S(Y) \simeq(Y-\langle Y\rangle)^{2} /\left(2 \sigma^{2}\right)$, where $\sigma^{2}=\left\langle(x-\langle x\rangle)^{2}\right\rangle$, if $Y$ is close to $\langle Y\rangle$.

Of course a) and b) are consequences of the law of large numbers, and c) is nothing but the central limit theorem. A mathematical introduction to the Large Deviation theory is given in [288].

\section{Appendix: Anosov systems}

Anosov systems play an important pedagogical role, in the theory of dynamical systems: their behaviour is highly chaotic and rich in structure. The celebrated Arnold cat map, defined by

$$
\left(\begin{array}{l}
x_{n+1} \\
y_{n+1}
\end{array}\right)=\left(\begin{array}{ll}
1 & 1 \\
1 & 2
\end{array}\right)\left(\begin{array}{l}
x_{n} \\
y_{n}
\end{array}\right) \bmod 1
$$

on the unit square $\mathcal{M}=[0,1] \times[0,1]$, belong to this class 27

Although they can be understood in rather simple terms, they are quite effective in expressing the concept of deterministic chaos. Anosov dynamics $S^{t}$ can be discrete (Anosov diffeomorphisms) or continuous (Anosov flows), and are defined on smooth manifolds $\mathcal{M}$ (the phase space). Anosov diffeomorphisms and flows are not completely equivalent, although from a flow one may always

\footnotetext{
${ }^{27}$ Because of the mod operation, the Arnold cat map is not a linear map.
} 
extract a map, observing the continuous time dynamics at discrete instants of time. Here, we limit our attention to properties which are similar in the two cases, presenting the general theory for continuous time systems, since physical systems mostly have continuous time, and illustrating it on the Arnold cat map, which can be explicitly worked out in detail.

For simplicity, assume that the dynamics explores densely $\mathcal{M}$, i.e. that it is transitive. Then, the main feature of an Anosov system is that $\mathcal{M}$ is hyperbolic, i.e. that for all $X \in \mathcal{M}$, the tangent space $T_{X} \mathcal{M}$ continuously splits in a stable, an unstable and a neutral linear space [290], $E_{X}^{s}, E_{X}^{u}$ and $E_{X}^{0}$ respectively. The tangent space of a flow at a point $X \in \mathcal{M}$ can be interpreted as the space of all possible velocities at that point. Indeed, the evolution remains in $\mathcal{M}$, hence these velocities can only be tangent to $\mathcal{M}$ in that point, and constitute a linear space of dimension equal to the dimension of $\mathcal{M}$. The points in $T_{X} \mathcal{M}$ are then also called tangent vectors. More precisely, let $D S^{t}(X)$ be the Jacobian map of the transformation $S^{t}$ (which evolves any initial condition $X$ into $X_{t}=S^{t} X$ ) evaluated at the point $X$. Clearly, $D S^{t}(X)$ depends on both $t$ and $X$. Then, for a flow, transitivity and hyperbolicity mean that:

a) $T_{X} \mathcal{M}=E_{X}^{u} \oplus E_{X}^{s} \oplus E_{X}^{0}$ is continuous (or smooth) in $X$, i.e. in any coordinate chart, the components of a constant vector field are continuous (or smooth) functions of the coordinates of the point $X . E_{X}^{0}$ is a one-dimensional linear subspace in case of flows, while it reduces to $\{0\}$ in case $S^{t}$ is a map.

b) The linear subspaces $E_{X}^{s}$ and $E_{X}^{u}$ are invariant with respect to the tangent (or Jacobian) map $D S^{t}$, i.e. for all $X \in \mathcal{M} D S^{t}(X) E_{X}^{s}=E_{S^{t}(X)}^{s}$ and $D S^{t}(X) E_{X}^{u}=E_{S^{t}(X)}^{u}$.

c) There exist $C>0$ and $1>\theta>0$, such that for all $t \in \mathbb{R}$, we have $\left\|D S^{t}(X) \xi\right\| \leq C \theta^{t}\|\xi\|$, for all $\xi \in E_{X}^{s}$ and $\left\|D S^{-t}(X) \eta\right\| \leq C \theta^{t}\|\eta\|$, for all $\forall \eta \in E_{X}^{u}$.

The tangent space at $X$ may also be seen as the space which contains the infinitesimal perturbations $\delta X=X^{\prime}-X$ of the initial point of a given trajectory, that produce nearby trajectories. Because $X$ and $X^{\prime}$ lie in $\mathcal{M}$, because $\mathcal{M}$ is assumed to be smooth, hence to have a tangent in $X$, and because $\delta X$ is infinitesimal, $\delta X$ lies in this tangent space, of dimension equal to that of $\mathcal{M}$. The tangent vectors evolve according to the rule $D S^{t}(X) \delta X$, hence condition c) implies that they all grow or decrease at exponential rates, which are everywhere bounded away from zero, except for the tangent vectors directed along the neutral space.

Furthermore, transitive Anosov systems enjoy the following properties [290,291]:

(1) the stable and unstable manifolds

$$
W_{X}^{s}=\left\{X^{\prime} \in \mathcal{M}: \lim _{t \rightarrow \infty}\left\|S^{t} X^{\prime}-S^{t} X\right\|=0\right\}
$$




$$
W_{X}^{u}=\left\{X^{\prime} \in \mathcal{M}: \lim _{t \rightarrow \infty}\left\|S^{-t} X^{\prime}-S^{-t} X\right\|=0\right\}
$$

are globally defined and dense in $\mathcal{M}$, for every $X \in \mathcal{M}$ (transitivity);

(2) periodic orbits are dense in $\mathcal{M}$;

(3) there is an invariant measure $\mu$ which represents the statistics of the forward time evolution, and which has a density along the unstable directions. Such a measure is usually called Sinai-Ruelle-Bowen (SRB) measure and satisfies

$$
\lim _{T \rightarrow \infty} \frac{1}{T} \int_{0}^{T} \Phi\left(S^{t} X\right)=\int \Phi(y) d \mu(y)=\langle\Phi\rangle
$$

for (Lebesgue) almost all $X \in \mathcal{M}$. For maps, the same holds, if the time integral is replaced by a sum over time steps.

Thanks to property (2), $\mu$ can be represented in terms of orbital measures. If $P(T, T+\epsilon]$ is the set of the unstable periodic orbits of the system, with period $\tau \in(T, T+\epsilon]$, for a fixed $\epsilon>0$ and for any $T>0$, the following holds $[292] 28$

Theorem:Let $\left(\mathcal{M}, S^{t}\right)$ be an Anosov flow (with $S^{t} \in C^{2}$ ). Let $\Phi \in C^{1}$, and let $j$ be an unstable periodic orbit of period $\tau_{j}$. For any $X_{j} \in j$, denote by $J_{j}^{u}$ the Jacobian of the dynamics restricted to the unstable manifold, with initial condition $X_{j}$. Then, for all $\epsilon>0$,

$$
\langle\Phi\rangle=\lim _{T \rightarrow \infty} \frac{\sum_{j \in P_{(T, T+\epsilon]}}\left(J_{j}^{u}\right)^{-1} \int_{0}^{\tau_{j}} \Phi\left(S^{t} X_{j}\right) d t}{\sum_{j \in P_{(T, T+\epsilon]}}\left(J_{j}^{u}\right)^{-1} \tau_{j}}
$$

where $X_{j}$ is any point in the orbit $j$.

A version of Eq.(C.2) is available for maps, and is formally identical to (C.2): it suffices to replace the integrals by sums. It is interesting to note that $\langle\Phi\rangle$ is obtained as a weighted average of orbital averages, with weights

$$
\left(J_{j}^{u}\right)^{-1} \cdot \tau_{j}
$$

which have the suggestive form "inverse of instability $\times$ period": i.e. one orbit contributes more than another to $\langle\Phi\rangle$ if it has larger period, but contributes less if it is more unstable. Furthermore, it has been observed that the neighbourhoods of the periodic orbits of a given interval of periods afford, at least in some systems, a hierarchical tessellation of the phase space, which is finer where $\mu$ is higher [293]. This should yield a rather quick convergence of the limit in (C.2), because the low period orbits cover the parts of phase space which have higher probability, leaving to the long period ones the task to

$\overline{28}$ This theorem holds more generally for Axiom-A systems, which may have a finite number of attracting sets. 
cover even the less probable parts. However, Eq.(C.2) has proved highly nontrivial to use for practical computations, and it has been tested and used only in low-dimensional systems, such as the Lorentz gas [294,295]. This does not prevent the use of that formula for theoretical calculations, hence various results of statistical mechanics interest have been obtained through it (see, e.g. $[190,296,191])$. Furthermore, it was the use of such weights that led the authors of [148] to propose the $\Omega$-FR (which happened to coincide with the $\Lambda$-FR in their case). This is why the mathematical formulation of the $\Lambda$-FR given by Gallavotti and Cohen was based on the Chaotic Hypothesis, i.e. on the hypothesis that systems of physical interest behave as if they were transitive Anosov systems.

All the above is easily illustrated by means of the Arnold cat map. Being a diffeomorphism, it has no neutral direction, and its tangent space at any point $X \in \mathcal{M}$ is $T_{X} \mathcal{M}=\mathbb{R}^{2}$. The eigenvalues and the eigenvectors of the matrix defining the map are $\lambda^{u, s}=(3 \pm \sqrt{5}) / 2$, and

$$
v^{u}=\left(\begin{array}{c}
1 \\
\frac{1}{2}(1+\sqrt{5})
\end{array}\right)=\left(\begin{array}{c}
1 \\
\phi
\end{array}\right), \quad v^{s}=\left(\begin{array}{c}
1 \\
\frac{1}{2}(1-\sqrt{5})
\end{array}\right)=\left(\begin{array}{c}
1 \\
-\phi^{-1}
\end{array}\right)
$$

where $\phi$ is the golden ratio. Therefore, points that lie close to $(0,0)$, in the straight line of slope $\phi$ passing through the point $(0,0)$ remain in that line and their distance grows by a factor $\phi$ at each iteration. When these points cross the boundary of the unit square, they re-enter the square from the opposite side, and continue to move on a line of same slope, while their distance continues to grow by the same factor, except for the effect produced by the mod operation. Therefore, the infinite line of slope $\phi$, passing through $(0,0)$, cut and moved by the mod 1 operation, constitutes the unstable manifold of the point $(0,0)$. Similarly, the line of slope $-\phi^{-1}$, passing through $(0,0)$, cut and moved by the mod 1 operation, constitutes the stable manifold of $(0,0)$, because its points are moved along that direction, while their distances are reduced by the factor $\phi^{-1}$ at every iteration of the map. Similarly, one finds that each point $X \in \mathcal{M}$ has stable and unstable manifolds which are the straight lines of slopes $\phi$ and $-\phi^{-1}$, passing through $X$, mod 1. The exponential growth and decrease of the sizes of the tangent vectors are precisely the factors $\phi$ and $\phi^{-1}$, because the tangent vectors are linear combinations of $v^{u}$ and $v^{s}$. The density of the unstable manifold of the point $(0,0)$, in the Arnold cat map, is easily understood observing that its slope is irrational. Therefore, if one thinks of it as the trajectory of a point particle, which moves at constant velocity, exits the square at given instants of times, and re-enters the square form the opposite side, one realizes that it can never re-enter at a point which has been previously visited. In other words, this trajectory, i.e. the unstable manifold, wraps around densely exploring all of $\mathcal{M}$. The invariant SRB measure is the Lebesgue measure $d \mu=d x d y$, and the density of the periodic orbits follows 
from the fact that the pieces of stable and unstable manifolds of a point intersect forming a grid (called Markov partition), whose cells have invariant borders, inside each of which there are periodic points. Because the manifolds are dense, hence can form arbitrarily fine grids, the density of the periodic points follows. To find the periodic points of period $n$, one must solve the equation

$$
\left(\begin{array}{l}
x \\
y
\end{array}\right)=\left(\begin{array}{ll}
1 & 1 \\
1 & 2
\end{array}\right)^{n}\left(\begin{array}{l}
x \\
y
\end{array}\right) \bmod 1
$$

For instance, $(0,0)$ is a fixed point, hence it is periodic of all periods. All the periodic orbits are unstable, because of the ubiquitous hyperbolic structure.

Normally, the Anosov systems that are considered to illustrate the idea of chaos in physics are low dimensional, like the Arnold cat, because their dimensionality is not particularly important for their statistical properties. These properties are due to the uniform hyperbolicity, i.e. to the violent and ubiquitous trend of the trajectories to separate at an exponential rate, which produces an extremely high degree of mixing in the phase space. This makes possible to formally obtain in low dimensional Anosov dynamics certain properties that are expected to hold in thermodynamic systems, like the symmetry of Onsager relations [189]. Obviously, the physical content of that symmetry in a system of one particle is totally different from the content of that symmetry in a thermodynamic system, and is obtained for completely different reasons. Adding that hardly any system of physical interest is of the Anosov kind, that the phase space dynamics is not the real space dynamics, and recalling the meaning of the statistical laws, as expressed e.g. in [141], cf. Sect.4.4.1, leads to the conclusion that some care must be used in describing physical phenomena by means of Anosov systems.

\section{Appendix: A pedagogical example}

It is very instructive, also from a pedagogical point of view, to work out, for a system simple enough, the connection between some of the results discussed in Sections 5, 6 and 7. We closely follow a recent interesting paper by Astumian [297], which considers the overdamped dynamics of a colloidal particle immersed in a fluid with viscosity $\gamma$, subject to a conservative force $F_{c}=-U^{\prime}(x)$ and pulled by a non-conservative force $F_{n c}(t)=g(t) F_{\text {ext }}$. The position $x$ of the particle obeys the Langevin equation

$$
\gamma \frac{d x}{d t}=F(t)+\sqrt{2 \gamma T} \eta(t)
$$

where $F(t)=F_{c}+F_{n c}(t), \eta(t)$ is a Gaussian white noise. This is also the equation that governs the process of pulling a terminal of a macromolecule 
anchored to a surface and surrounded by water; this system has been studied in recent experiments [256,265].

Following the discussion of Onsager-Machlup theory in Sec. 7.1, in particular the non-linear generalization of Par. 7.1.1, one can obtain the probability density of a path starting at time $t_{0}$ and ending at time $t$. Again it is sufficient to consider discrete times $t_{0}+k \tau$ with $\tau$ arbitrarily small and $k \in[0, n]$ with $n$ being the integer part of $\left(t-t_{0}\right) / \tau$. Since the noise is Gaussian and delta-correlated, the sequence of variables $\eta_{k}=\eta\left(t_{0}+k \tau\right)$ has the probability density

$$
P\left[\left(\eta_{n}, t|\ldots| \eta_{0}, 0\right)\right] \propto \exp \left(-\frac{1}{2} \sum_{k=0}^{n} \eta_{k}^{2} \tau\right)
$$

which, in the limit $\tau \rightarrow 0$, becomes

$$
P\left[\left(\eta_{n}, t|\ldots| \eta_{0}, 0\right)\right] \propto \exp \left(-\frac{1}{2} \int_{0}^{t} d s \eta^{2}(s)\right) .
$$

Equation (D.1) tells us that $\eta(t)=\left(\frac{d x}{d t}-F / \gamma\right) / \sqrt{2 D}$, with $D=T / \gamma$, which finally gives us

$$
P[\{\eta(t)\}] \propto \exp (-L)
$$

where

$$
L=\frac{1}{4 D} \int_{0}^{t} d s\left(\frac{d x}{d s}-\frac{F}{\gamma}\right)^{2}=\frac{\Delta U-w_{e x t}[\{\eta(t)\}]}{2 \gamma D}+\frac{1}{4 D} \int_{0}^{t} d s\left[\left(\frac{d x}{d s}\right)^{2}+\frac{F^{2}}{\gamma^{2}}\right]
$$

is the thermodynamic action,

$$
w_{\text {ext }}[\{\eta(t)\}]=\int_{0}^{t} d s\left(F_{n c}(s) \frac{d x}{d s}\right)
$$

is the work done by the external forcing $F_{n c}(t)$ and $\Delta U=-\int_{x_{0}}^{x_{t}} F_{c}(x) d x$ is the difference of potential between the final and the initial position. To find the most probable path from $\left(x_{0}, 0\right)$ to $\left(x_{t}, t\right)$, it is sufficient to minimize the action (D.5) while keeping fixed the endpoints. Note that to obtain, from Eq. (D.4), the correct probability density for the trajectories $x(t)$, one has to calculate the Jacobian of the transformation $\eta(t) \rightarrow x(t)$. Anyway, even disregarding this problem, many interesting results discussed in this review can already be reproduced.

- A difference (asymmetry) between the probability of a path $\{\eta(t)\}$ and its time-reversed $\{I \eta(t)\}$, follows from the fact that the last integral in (D.5) does not change sign upon applying the time-reversal operator $I$. This corresponds, in this example, to the asymmetry of paths discussed in 7.2.1. Of course the richness of the recent results in Sections 7.2 and 7.3 (such as the non-locality of the entropy functional), which concerns spatially extended systems (fields) cannot be reproduced by this example. 
- The ratio between the probability density of a path and that of its timereversed reads:

$$
\frac{P[\eta(t)]}{P[\operatorname{I\eta }(t)]}=\exp \left(\frac{w_{e x t}[\eta(t)]-\Delta U}{k_{B} T}\right),
$$

which, for large times, allows one to identify the work $w_{\text {ext }}$ done by the external non-conservative force (divided by $k_{B} T$ ) as the entropy produced during the time $t$. This is an example of the result by Kurchan [164] and by Lebowitz and Spohn [227] on the FR for stochastic systems. Even without referring to the FR, Eq. (D.7) is interesting because the r.h.s. does not depend explicitly on time.

- By a simple calculation it is immediate to see that

$$
\frac{P\left(w_{e x t}[\eta(t)]=W\right)}{P\left(w_{e x t}[\eta(t)]=-W\right)}=\exp \left(\frac{W}{k_{b} T}\right)
$$

which is the FR for stochastic systems.

- Finally, one has

$$
\left\langle\exp \left(\frac{-W}{k_{B} T}\right)\right\rangle=\int_{-\infty}^{\infty} d W \exp \left(\frac{-W}{k_{B} T}\right) P(W)=\int_{-\infty}^{\infty} d W P(-W)=1
$$

which is nothing but the Jarzynski relation, or the partition identity, in this steady state case.

\section{References}

[1] E. Marinari, G. Parisi, F. Ricci-Tersenghi, J. Ruiz-Lorenzo, Violation of the fluctuation-dissipation theorem in finite-dimensional spin glasses, J. Phys. A. 31 (1998) 2611.

[2] A. Crisanti, F. Ritort, Violation of the fluctuation-dissipation theorem in glassy systems: basic notions and the numerical evidence, J. Phys. A 36 (2003) R181.

[3] L. Leuzzi, T. M. Nieuwenhuizen, Thermodynamics of the Glassy State, Taylor \& Francis, 2007.

[4] E. Mach, The Science of Mechanics, Open Court, Chicago, 1883.

[5] L. Boltzmann, Lectures on Gas Theory, Dover, 1995 (1896 first german edition).

[6] J. W. Gibbs, Elementary Principles in Statistical Mechanic, Yale University Press, 1902.

[7] A. Einstein, Zur allgemeinen molekularen theorie der wärme, Ann. der Phys. 14 (1904) 354. 
[8] J. Mehra, The Golden Age of Theoretical Physics, World Scientific, Singapore, 2001.

[9] A. Einstein, On the movement of small particles suspended in a stationary liquid demanded by the molecular-kinetic theory of heat, Ann. d. Phys. 17 (1905) 549.

[10] A. Einstein, On the theory of the brownian movement, Ann. d. Phys. 19 (1906) 371.

[11] M. Smoluchowski, Zur kinetischen theorie der brownschen molekularbewegung und der suspensionen, Ann. d. Phys. 21 (1906) 756.

[12] P. Langevin, Sur la theorie du mouvement brownien, C. R. Acad. Sci. (Paris) 146 (1908) 530, translated in Am. J. Phys. 65, 1079 (1997).

[13] C. Gardiner, Handbook of Stochastic Methods for Physics, Chemistry and the Natural Sciences, Springer- Verlag, Berlin, 1990.

[14] J. Perrin, Les atomes, Alcan, Paris, 1913.

[15] A. Munster, Classical Thermodynamics, John Wiley and Sons, 1970.

[16] M. Tuckerman, Lecture notes on statistical mechanics, http://www.nyu.edu/classes/tuckerman/stat.mech/lectures.html.

[17] D. J. Evans, G. P. Morriss, Statistical Mechanics of Nonequilibrium Liquids, Academic Press, 1990.

[18] D. A. McQuarrie, Statistical Mechanics, University Science Books, 2000.

[19] J. Keizer, Statistical Thermodynamics of Nonequilibrium Processes, SpringerVerlag, Berlin, 1987.

[20] E. A. Novikov, Functionals and the random-force method in turbulence theory, Soviet Physcis-JETP 20 (1965) 1290.

[21] J. Johnson, Thermal agitation of electricity in conductors, Phys. Rev. 32 (1928) 97.

[22] H. Nyquist, Thermal agitation of electric charge in conductors, Phys. Rev. 32 (1928) 110-113.

[23] D. Topaj, W. Kye, A. Pikovsky, Transition to coherence in populations of coupled chaotic oscillators: a linear response approach, Phys. Rev. Lett. 87 (2001) 074101.

[24] L. Onsager, Reciprocal relations in irreversible processes. I, Phys. Rev. 37 (1931) 405-426.

[25] L. Onsager, Reciprocal relations in irreversible processes. II, Phys. Rev. 38 (1931) 2265-2279.

[26] S. R. de Groot, P. Mazur, Non-equilibrium thermodynamics, Dover Publications, New York, 1984. 
[27] R. Kubo, The fluctuation-dissipation theorem, Rep. Prog. Phys. 29 (1966) 255.

[28] R. Kubo, Brownian motion and nonequilibrium statistical mechanics, Science 32 (1986) 2022.

[29] U. Deker, F. Haake, Fluctuation-dissipation theorems for classical processes, Phys. Rev. A 11 (1975) 2043.

[30] P. Hänggi, H. Thomas, Time evolution, correlations and linear response of non-markov processes, Z. Physik B 26 (1977) 85.

[31] P. Hänggi, H. Thomas, Stochastic processes: Time-evolution, symmetries and linear response, Phys. Rep. 88 (1982) 207.

[32] M. Falcioni, S. Isola, A. Vulpiani, Correlation functions and relaxation properties in chaotic dynamics and statistical mechanics, Physics Letters A 144 (1990) 341.

[33] R. H. Kraichnan, Classical fluctuation-relaxation theorem, Phys. Rev. 113 (1959) 118.

[34] R. H. Kraichnan, Deviations from fluctuation-relaxation relations, Physica A 279 (2000) 30.

[35] G. Boffetta, G. Lacorata, S. Musacchio, A. Vulpiani, Relaxation of finite perturbations: Beyond the fluctuation-response relation, Chaos 13 (2003) 806.

[36] N. van Kampen, The case against linear response theory, Phys. Norv. 5 (1971) 279 .

[37] G.Paladin, A.Vulpiani, Anomalous scaling laws in multifractal objects, Phys. Rep. 156 (1987) 147.

[38] D. Ruelle, General linear response formula in statistical mechanics, and the fluctuation-dissipation theorem far from equilibrium, Phys. Letters A 245 (1998) 220.

[39] B. Cessac, J.-A. Sepulchre, Linear response, susceptibility and resonance in chaotic toy models, Physica D 225 (2007) 13.

[40] B. Alder, D. Gass, T. Wainwright, Studies in molecular dynamics. VIII. Transport coefficients for a hard- sphere fluid, J. Chem. Phys. 53 (1970) 3813.

[41] D. Levesque, L. Verlet, J. Kürkijarvi, Computer "experiments" on classical fluids. IV. Transport properties and time-correlation functions on the LennardJones liquid near its triple point, Phys. Rev. A 7 (1973) 1690.

[42] G. Ciccotti, G. Jacucci, Direct computation of dynamical response by molecular dynamics: The mobility of charged Lennard- Jones particles, Phys. Rev. Lett. 35 (1975) 789.

[43] G. Jacucci, Linear and nonlinear response in computer simulation experiments, Physica A 118 (1983) 157. 
[44] G. Ciccotti, G. Jacucci, I. McDonald, "Though- Experiments" by molecular dynamics, J. Stat. Phys. 21 (1979) 1.

[45] G. Morris, D. Evans, E. Cohen, H. van Beijeren, Linear response in phase-space trajectories to shearing, Phys. Rev. Lett. 62 (1989) 1579.

[46] G. Boffetta, M. Cencini, M. Falcioni, A. Vulpiani, Predictability: a way to characterize complexity, Phys. Rep. 356 (2002) 367.

[47] G. Carnevale, M. Falcioni, S. Isola, R. Purini, A. Vulpiani, Fluctuationresponse relation in systems with chaotic behaviour, Phys. Fluids A 3 (1991) 2247.

[48] E. Ott, Chaos in Dinamical Systems, Cambridge University Press, Cambridge U.K., 1993.

[49] M. Falcioni, L. Palatella, A. Vulpiani, Production rate of the coarse-grained Gibbs entropy and the Kolmogorov-Sinai entropy: A real connection?, Phys. Rev. E 71 (2005) 016118.

[50] A. I. Khinchin, Mathematical Foundations of Statistical Mechanics, Dover Publications Inc., New York, 1949.

[51] P. Mazur, J. van der Linden, Asymptotic form of the structure function for real systems, J. Math. Phys. 4 (1963) 271.

[52] R. Kubo, Statistical-mechanical theory of irreversible processes. I. General theory and simple applications to magnetic and conduction problems, J. Phys. Soc. Japan 12 (1957) 570.

[53] M. H. Lee, Ergodic theory, infinite products, and long time behavior in hermitian models, Phys. Rev. Lett. 87 (2001) 250601.

[54] M. H. Lee, Ergodicity in simple and not so simple systems and Kubo's condition, Physica A 314 (2002) 583.

[55] M. H. Lee, Why does Boltzmann's ergodic hypothesis work and when does it fail, Physica A 365 (2006) 150.

[56] M. H. Lee, Why irreversibility is not a sufficient condition for ergodicity, Phys. Rev. Lett 98 (2007) 190601.

[57] M. H. Lee, Birkhoff's theorem, many-body response functions and the ergodic condition, Phys. Rev. Lett. 98 (2007) 110403.

[58] G. M. Zaslavsky, D. Stevens, H. Weitzner, Self-similar transport in incomplete chaos, Phys. Rev. E 48 (1993) 1683.

[59] P. Castiglione, A. Mazzino, P. Muratore-Ginanneschi, A. Vulpiani, On strong anomalous diffusion, Physica D 134 (1999) 75.

[60] R. Metzler, E. Barkai, J. Klafter, Anomalous diffusion and relaxation close to thermal equilibrium: A fractional Fokker-Planck equation approach, Phys. Rev. Lett 82 (1999) 3563. 
[61] Q. Gu, E. A. Schiff, S. Grebner, F. Wang, R. Schwarz, Non-gaussian transport measurements and the Einstein relation in amorphous silicon, Phys. Rev. Lett. 76 (1996) 3196.

[62] P. Leboeuf, Normal and anomalous diffusion in a deterministic area-preserving map, Physica D 116 (1998) 8.

[63] O. G. Jepps, L. Rondoni, Thermodynamics and complexity of simple transport phenomena, J. Phys. A 39 (2006) 1311.

[64] L. Biferale, A. Crisanti, M. Vergassola, A. Vulpiani, Eddy diffusivities in scalar transport, Phys. Fluids 7 (1995) 2725.

[65] G. Trefan, E. Floriani, B. J. West, P. Grigolini, Dynamical approach to anomalous diffusion: Response of Levy processes to a perturbation, Phys. Rev. E 50 (1994) 2564.

[66] R. Rose, P. Sulem, Fully developed turbulence and statistical mechanics, J. Phys. (Paris) 39 (1978) 441.

[67] R. H. Kraichnan, Dynamics of nonlinear stochastic systems, J. Math. Phys. 2 (1961) 124.

[68] W. McComb, K. Kiyani, Eulerian spectral closures for isotropic turbulence using a time-ordered fluctuation-dissipation relation, Physical Review E 72 (2005) 016309.

[69] U. Frisch, Turbulence, Cambridge University Press, Cambridge U.K., 1995.

[70] C. E. Leith, Climate response and fluctuation dissipation, J. Atmos. Sci. 32 (1975) 2022.

[71] C. E. Leith, Predictability of climate, Nature 276 (1978) 352.

[72] R. Bell, Climate sensitivity from fluctuation dissipation: Some simple model tests, J. Atmos. Sci. 37 (1980) 1700.

[73] T. Bohr, M. Jensen, G. Paladin, A. Vulpiani, Dynamical Systems Approach to Turbulence, (Cambridge University Press, Cambridge U.K., 1998.

[74] S. A. Orszag, G. S. Patterson, Numerical simulation of three-dimensional homogeneous isotropic turbulence, Phys. Rev. Lett. 28 (1972) 76.

[75] C. E. Seyler, Y. Salu, D. Montgomery, G. Knorr, Two-dimensional turbulence in inviscid fluids or guiding center plasmas, Phys. Fluids 18 (1975) 803.

[76] R. Robert, Statistical equilibrium states for 2-dimensional flows, J. of Fluid Mech. 229 (1991) 291.

[77] R. A. Pasmanter, On long-lived vortices in 2-D viscous flows, most probable states of inviscid 2-D flows and a soliton equation, Physics of Fluids 6 (1994) 1236 .

[78] R. Balescu, Equilibrium and Nonequilibrium Statistical Mechanics, J. Wiley and Sons, 1975. 
[79] M. Lesieur, Turbulence in Fluids, Kluwer Academic Publishers, Dordrecht, 1990.

[80] R. H. Kraichnan, Isotropic turbulence and inertial-range structure, Physics of Fluids 9 (1966) 1728.

[81] G. Eyink, Large-N limit of the "spherical model" of turbulece, Phys. Rev. E 49 (1994) 3990.

[82] A. Koniges, C. Leith, Statistical closure approximation and the fluctuationdissipation theorem for drift-wave interaction, Physics of Fluids 30 (1987) 3065.

[83] K. Kiyani, W. McComb, Time-ordered fluctuation-dissipation relation for incompressible isotropic turbulence, Physical Review E 70 (2004) 066303.

[84] S. Orszag, J. McLaughlin, Evidence that random behavior is generic for nonlinear differential equations, Physica D 1 (1980) 68.

[85] L. Biferale, I. Daumont, G. Lacorata, A. Vulpiani, Fluctuation-response relation in turbulent systems, Phys. Rev. E 65 (2002) 016302.

[86] L. Biferale, Shell models of energy cascade in turbulence, Ann. Rev. of Fluid Mech. 35 (2003) 441.

[87] V. L'vov, E. Podivilov, A. Pomyalov, A. Procaccia, D. Vandembroucq, Improved shell model of turbulence, Phys. Rev. E 58 (1998) 1811.

[88] E. Lorenz, Deterministic non-periodic flow, J. Atmos. Sci. 20 (1963) 130.

[89] P. Ditlevsen, Observation of $\alpha$-stable noise induced millennial climate changes from an ice-core record, Geophys. Res. Lett. 26 (1999) 1441.

[90] G. North, R. Bell, J. Hardin, Fluctuation dissipation in a general circulation model, Climate Dynamics 8 (1993) 259.

[91] I. Cionni, G. Visconti, F. Sassi, Fluctuation dissipation theorem in a general circulation model, Geophys. Res. Lett. 31 (2004) L09206.

[92] R. Kaskins, R. Goody, L. Chen, Radiance covariance and climate models, J. of Climate 12 (1999) 1408.

[93] G. Lacorata, A. Vulpiani, Fluctuation-response relation and modeling in systems with fast and slow dynamics, Nonlin. Processes Geophys. 14 (2007) 681.

[94] H. M. Jaeger, S. R. Nagel, Physics of the granular state, Science 255 (1992) 1523.

[95] H. M. Jaeger, S. R. Nagel, R. P. Behringer, The physics of granular materials, Physics Today 49 (April) (1996) 32.

[96] H. M. Jaeger, S. R. Nagel, R. P. Behringer, Granular solids, liquids, and gases, Reviews of Modern Physics 68 (1996) 1259. 
[97] G. D'Anna, P. Mayor, G. Gremaud, A. Barrat, V. Loreto, F. Nori, Observing brownian motion in vibration-fluidized granular matter, Nature 424 (2003) 909.

[98] M. Nicodemi, A. Coniglio, Aging in out-of-equilibrium dynamics of models for granular media, Phys. Rev. Lett. 82 (1999) 916.

[99] A. Barrat, V. Loreto, Response properties in a model for granular matter, J. Phys. A 33 (2000) 4401.

[100] C. Joserand, A. V. Tkachenko, D. M. Mueth, H. M. Jaeger, Memory effects in granular materials, Phys. Rev. Lett 85 (2000) 3632.

[101] A. Barrat, V. Loreto, Memory in aged granular media, Europhys. Lett. 53 (2001) 297.

[102] A. Barrat, V. Colizza, V. Loreto, Fluctuation-dissipation ratio for compacting granular media, Phys. Rev. E 66 (2002) 011310.

[103] J. W. Dufty, Statistical mechanics, kinetic theory, and hydrodynamics for rapid granular flow, Journal of Physics: Condensed Matter 12 (2000) A47.

[104] J. J. Brey, J. W. Dufty, C. S. Kim, A. Santos, Hydrodynamics for granular flow at low density, Phys. Rev. E 58 (4) (1998) 4638.

[105] N. V. Brilliantov, T. Poschel, Self-diffusion in granular gases: Green-Kubo versus Chapman-Enskog, Chaos 15 (2005) 026108.

[106] L. P. Kadanoff, Built upon sand: Theoretical ideas inspired by granular flows, Rev. Mod. Phys. 71 (1999) 435.

[107] I. Goldhirsch, Scales and kinetics of granular flows, Chaos 9 (1999) 659.

[108] J. W. Dufty, V. Garzó, Mobility and diffusion in granular fluids, J. Stat. Phys. 105 (5) (2001) 723.

[109] V. Garzó, J. M. Montanero, Diffusion of impurities in a granular gas, Physical Review E 69 (2004) 021301.

[110] J. J. Brey, M. J. Ruiz-Montero, Simulation study of the Green-Kubo relations for dilute granular gases, Physical Review E 70 (2004) 051301.

[111] J. W. Dufty, J. J. Brey, Green-Kubo expressions for a granular gas, J. Stat. Phys. 109 (2002) 433.

[112] J. Dufty, A. Baskaran, J. J. Brey, Linear response for granular fluids, Journal of Statistical Mechanics: Theory and Experiment (2006) L08002.

[113] J. J. Brey, M. J. Ruiz-Montero, D. Cubero, Homogeneous cooling state of a low-density granular flow, Phys. Rev. E 54 (1996) 3664.

[114] P. K. Haff, Grain flow as a fluid-mechanical phenomenon, J. Fluid. Mech. 134 (1983) 401. 
[115] A. Puglisi, A. Baldassarri, V. Loreto, Fluctuation-dissipation relations in driven granular gases, Physical Review E 66 (2002) 061305.

[116] A. Barrat, V. Loreto, A. Puglisi, Temperature probes in binary granular gases, Physica A 334 (2004) 513.

[117] A. Baldassarri, A. Barrat, G. D'Anna, V. Loreto, P. Mayor, A. Puglisi, What is the temperature of a granular medium?, Journal of Physics: Condensed Matter 17 (2005) S2405.

[118] A. Puglisi, A. Baldassarri, A. Vulpiani, Violations of the Einstein relation in granular fluids: the role of correlations, J. Stat. Mech. (2007) P08016.

[119] V. Garzó, On the Einstein relation in a heated granular gas, Physica A 343 (2004) 105.

[120] P. Melby, A. Prevost, D. A. Egolf, J. S. Urbach, The depletion force in a bi-disperse granular layer, Phys. Rev. E 76 (2007) 051307.

[121] Y. Srebro, D. Levine, Exactly solvable model for driven dissipative systems, Phys. Rev. Lett. 93 (2004) 240601.

[122] Y. Shokef, G. Bunin, D. Levine, Fluctuation-dissipation relations in driven dissipative systems, Phys. Rev. E 73 (2006) 046132.

[123] D. Levanony, D. Levine, Correlation and response in a driven dissipative model, Phys. Rev. E 73 (2006) 055102(R).

[124] A. Baldassarri, U. M. B. Marconi, A. Puglisi, Influence of correlations on the velocity statistics of scalar granular gases, Europhys. Lett. 58 (2002) 14.

[125] A. Baldassarri, U. M. B. Marconi, A. Puglisi, Cooling of a lattice granular fluid as an ordering process, Phys. Rev. E 65 (2002) 051301.

[126] M. H. Ernst, R. Brito, High-energy tails for inelastic maxwell models, Europhys. Lett. 58 (2002) 182.

[127] R. Pagnani, U. M. B. Marconi, A. Puglisi, Driven low density granular mixtures, Phys. Rev. E 66 (2002) 051304.

[128] U. M. B. Marconi, A. Puglisi, Steady-state properties of a mean-field model of driven inelastic mixtures, Phys. Rev. E 66 (2002) 011301.

[129] U. M. B. Marconi, A. Puglisi, Mean-field model of free-cooling inelastic mixtures, Phys. Rev. E 65 (2002) 051305.

[130] A. Barrat, E. Trizac, Lack of energy equipartition in homogeneous heated binary granular mixtures, Granular Matter 4 (2002) 57.

[131] K. Feitosa, N. Menon, Breakdown of energy equipartition in a 2d binary vibrated granular gas, Phys. Rev. Lett. 88 (2002) 198301.

[132] P. A. Martin, J. Piasecki, Thermalization of a particle by dissipative collisions, Europhys. Lett. 46 (1999) 613. 
[133] A. Puglisi, V. Loreto, U. M. B. Marconi, A. Petri, A. Vulpiani, Clustering and non-gaussian behavior in granular matter, Phys. Rev. Lett. 81 (1998) 3848.

[134] A. Puglisi, V. Loreto, U. M. B. Marconi, A. Vulpiani, A kinetic approach to granular gases, Phys. Rev. E 59 (1999) 5582.

[135] F. Cecconi, F. Diotallevi, U. M. B. Marconi, A. Puglisi, Fluid-like behavior of a one-dimensional granular gas, J. Chem. Phys. 120 (2004) 35.

[136] U. M. B. Marconi, P. Tarazona, F. Cecconi, Theory of thermostatted inhomogeneous granular fluids: a self-consistent density functional description, J. Chem. Phys. 126 (2007) 164904.

[137] D. R. M. Williams, F. C. MacKintosh, Driven granular media in one dimension: Correlations and equation of state, Phys. Rev. E 54 (1996) R9.

[138] T. P. C. van Noije, M. H. Ernst, E. Trizac, I. Pagonabarraga, Randomly driven granular fluids: Large-scale structure, Phys. Rev. E 59 (1999) 4326.

[139] E. Trizac, I. Pagonabarraga, T. P. C. van Noije, M. H. Ernst, Randomly driven granular fluids: Collisional statistics and short scale structure, Phys. Rev. E 65 (2001) 011303.

[140] T. Speck, U. Seifert, Restoring a fluctuation-dissipation theorem in a nonequilibrium steady state, Europhys. Lett. 74 (2006) 391.

[141] L. D. Landau, E. M. Lifchitz, Physique Statistique, Éditions MIR, 1967.

[142] C. Bustamante, J. Liphardt, F. Ritort, The nonequilibrium thermodynamics of small systems, Physics Today 58 (2005) 43.

[143] F. Ritort, Nonequilibrium fluctuations in small systems: from physics to biologyArXiv:0705.0455.

[144] T. A. Desai, D. Hansford, M. Ferrari, Characterization of micromachined silicon membranes for immunoisolation and bioseparation applications, J. Membrane Sci. 159 (1999) 221.

[145] K. Sato, Y. Ito, T. Yomo, K. Kaneko, On the relation between fluctuation and response in biological systems, Proc. Nat. Acad. Sci. 100 (2003) 14086.

[146] K. Kaneko, C. Furusawa, An evolutionary relationship between genetic variation and phenotypic fluctuation, J. Theor. Biol. 240 (2006) 78.

[147] R. A. Fisher, The genetical theory of natural selection, Oxford University Press, Oxford, 1999.

[148] D. J. Evans, E. G. D. Cohen, G. P. Morriss, Probability of second law violations in shearing steady flows, Phys. Rev. Lett. 71 (1993) 2401.

[149] G. Gallavotti, Ergodicity, ensembles, irreversibility in Boltzmann and beyond, J. Stat. Phys 78 (1995) 1571.

[150] G. Gallavotti, E. G. D. Cohen, Dynamical ensembles in stationary states, J. Stat. Phys. 80 (1995) 931. 
[151] D. J. Evans, D. J. Searles, The fluctuation theorem, Adv. Phys. 52 (2002) 1529.

[152] G. Gallavotti, Heat and fluctuations from order to chaos, arXiv:0711.2755 (2007).

[153] R. Chetrite, K. Gawedzki, Fluctuation relations for diffusion processes, arXiv:0707.2725 (2007).

[154] D. J. Evans, D. J. Searles, Steady states, invariant measures, response theory, Phys. Rev. E 52 (1995) 5839.

[155] D. J. Searles, G. Ayton, D. J. Evans, Generalised fluctuation formula, AIP Conference Series 519 (2000) 271.

[156] D. J. Searles, D. J. Evans, Ensemble dependence of the transient fluctuation theorem, J. Chem. Phys. 113 (2000) 3503.

[157] S. R. Williams, D. J. Searles, D. J. Evans, Thermostat invariance of the transient fluctuation theorem, Phys. Rev. E 70 (2004) 066113.

[158] S. R. Williams, D. J. Searles, D. J. Evans, Numerical study of the steady state fluctuation relations far from equilibrium, J. Chem. Phys. 124 (2006) 194102.

[159] G. Gallavotti, Reversible Anosov diffeomorphisms, large deviations, Math. Phys. Electronic J. 1 (1995) 1.

[160] G. Gallavotti, Fluctuation theorem revisited, cond-mat/0404699 (2004).

[161] D. Evans, D. Searles, L. Rondoni, Application of the Gallavotti-Cohen fluctuation relation to thermostatted steady states near equilibrium, Phys. Rev. E 71 (2005) 056120.

[162] F. Bonetto, G. Gallavotti, A. Giuliani, F. Zamponi, Chaotic hypothesis, fluctuation theorem, singularities, J. Stat. Phys. 123 (2006) 39.

[163] C. Jarzynski, Nonequilibrium equality for free energy differences, Phys. Rev. Lett. 78 (1997) 2690.

[164] J. Kurchan, Fluctuation theorem for stochastic dynamics, J. Phys. A 31 (1998) 3719 .

[165] R. van Zon, E. G. D. Cohen, Extension of the fluctuation theorem, Phys. Rev. Lett. 91 (2003) 110601.

[166] T. Bodineau, B. Derrida, Current fluctuations in nonequilibrium diffusive systems: an additivity principle, Phys. Rev. Lett. 92 (2004) 180601.

[167] L. Bertini, A. D. Sole, D. Gabrielli, G. Jona-Lasinio, C. Landim, Current fluctuations in stochastic lattice gases, Phys. Rev. Lett. 94 (2005) 030601.

[168] A. Khinchin, Mathematical Foundations of Statistical Mechanics, Dover Publications, New York, 1949. 
[169] P. Mazur, J. van der Linden, Asymptotic form of the structure function for real systems, J. Math. Phys. 4 (1963) 271.

[170] W. G. Hoover, Computational Statistical Mechanics, Elsevier, 1991.

[171] C. Lanczos, The Variational Principles of Mechanics, Dover, New York, 1979.

[172] S. Sarman, D. J. Evans, P. T. Cummings, Recent developments in nonNewtonian molecular dynamics, Physics Reports 305 (1998) 1.

[173] D. Ruelle, Conversations on nonequilibrium physics with an extraterrestrial, Physics Today 57 (2004) 48.

[174] E. G. D. Cohen, L. Rondoni, Note on phase space contraction and entropy production in thermostatted hamiltonian systems, Chaos 8 (1998) 357.

[175] D. J. Evans, G. P. Morriss, Equilibrium time correlation-functions under Gaussian isothermal dynamics, Chem. Phys. 87 (1984) 451.

[176] S. Y. Liem, D. Brown, J. H. R. Clarke, Investigation of the homogeneous-shear nonequilibrium-molecular-dynamics method, Phys. Rev. A 45 (1992) 3706.

[177] D. J. Evans, S. Sarman, Equivalence of thermostatted nonlinear responses, Phys. Rev. E 48 (1993) 65.

[178] W. G. Hoover, K. Aoki, C. G. Hoover, S. V. D. Groot, Time-reversible deterministic thermostats, Physica D 187 (2004) 253.

[179] H. A. Posch, W. G. Hoover, Large-system phase-space dimensionality loss in stationary heat flows, Physica D 187 (2004) 281.

[180] K. Aoki, D. Kusnezov, Lyapunov exponents, the extensivity of dimensional loss for systems in thermal gradients, Phys. Rev. E 68 (2003) 056204.

[181] T. Tel, J. Vollmer, W. Breymann, Transient chaos: The origin of transport in driven systems, Europhys. Lett. 35 (1996) 659.

[182] G. Gallavotti, Dynamical ensemble equivalence in fluid mechanics, Physica D 105 (1997) 163.

[183] G. Gallavotti, L. Rondoni, E. Segre, Lyapunov spectra, nonequilibrium ensembles equivalence in 2D fluid mechanics, Physica D 187 (2004) 338.

[184] G. Gentile, Large deviations for Anosov flows, Forum Math. 10 (1998) 89.

[185] Y. G. Sinai, Lectures in ergodic theory, Princeton Univ. Press, Princeton, 1977.

[186] D. J. Evans, E. G. D. Cohen, D. J. Searles, F. Bonetto, Note on the KaplanYorke dimension and linear transport coefficients, J. Stat. Phys. 101 (2000) 17 .

[187] G. Gallavotti, Extension of Onsager's reciprocity to large fields, the chaotic hypothesis, Phys. Rev. Lett. 77 (1996) 4334. 
[188] D. Ruelle, Differentiation of srb states: correction and complements, Comm. Math. Phys. 234 (2003) 185-190.

[189] G. Gallavotti, D. Ruelle, SRB states and nonequilibrium statistical mechanics close to equilibrium, Comm. Math. Phys. 190 (1997) 279.

[190] L. Rondoni, E. G. D. Cohen, Orbital measures in non-equilibrium statistical mechanics: the Onsager relations, Nonlinearity 11 (1998) 1395.

[191] L. Rondoni, Deterministic thermostats, fluctuation relations, in: P. Garbaczewski, R. Olkiewicz (Eds.), Dynamics of dissipation, Lecture Notes in Physics 597, Springer Verlag, 2002.

[192] L. Rondoni, T. Tél, J. Vollmer, Fluctuation theorems for entropy production in open systems, Phys. Rev. E 61 (2000) R4679.

[193] C. Maes, F. Redig, M. Verschuere, From global to local fluctuation theorems, Moscow Math. J. 1 (2001) 421.

[194] G. Gallavotti, A local fluctuation theorem, J. Phys. A 263 (1999) 39.

[195] G. Gallavotti, Fluctuation patterns and conditional reversibility in nonequilibrium system, Ann. Inst. H. Poincaré 70 (1999) 429.

[196] G. Gallavotti, Large deviations, fluctuation theorem, Onsager-Machlup theory in nonequilibrium statistical mechanics, http://ipparco.roma1.infn.it/.

[197] M. Dolowschiak, Z. Kovacs, Fluctuation formula in the Nosé-Hoover thermostated Lorentz gas, Phys. Rev. E 71 (2005) 025202.

[198] F. Zamponi, G. Ruocco, L. Angelani, Fluctuations of entropy production in the isokinetic ensemble, J. Stat. Phys. 115 (2004) 1655.

[199] D. J. Evans, A non-equilibrium free energy theorem for deterministic systems, Molecular Phys. 101 (2003) 1551.

[200] R. van Zon, E. G. D. Cohen, Stationary and transient work-fluctuation theorems for a dragged Brownian particle, Phys. Rev. E 67 (2003) 046102.

[201] D. J. Searles, L. Rondoni, D. J. Evans, The steady state fluctuation relation for the dissipation function, J. Stat. Phys. 128 (2007) 1337.

[202] D. J. Evans, D. J. Searles, Equilibrium microstates which generate second law violating steady states, Phys. Rev. E 50 (1994) 1645.

[203] D. J. Searles, L. Rondoni, D. J. Evans, in preparation.

[204] D. J. Evans, D. J. Searles, The dissipation theorem, (submitted) (2007).

[205] E. G. D. Cohen, D. Mauzerall, A note on the Jarzynski equality, J. Stat. Mech. (2004) P07006.

[206] D. J. Evans, A non-equilibrium free energy theorem for deterministic systems, Mol. Phys. 101 (2003) 1551. 
[207] G. E. Crooks, Path ensemble averages in systems driven far from equilibrium, Phys. Rev. E 61 (2000) 2361.

[208] B. Cleuren, C. V. den Broeck, R. Kawai, Work, dissipation and fluctuations in nonequilibrium physics, Comptes Rendus Physique 8 (2007) 567.

[209] J. Kurchan, Nonequilibrium work relations, cond-mat/0511073 (2005).

[210] J. L. Lebowitz, H. Spohn, A Gallavotti-Cohen-type symmetry in the large deviation functional for stochastic dynamics, J. Stat. Phys. 95 (1999) 333.

[211] C. Maes, The fluctuation theorem as a Gibbs property, J. Stat. Phys. 95 (1999) 367.

[212] J. Farago, Injected power fluctuations in Langevin equation, J. Stat. Phys. 107 (2002) 781.

[213] G. M. Wang, E. M. Sevick, E. Mittag, D. J. Searles, D. J. Evans, Experimental demonstration of violations of the second law of thermodynamics for small systems and short time scales, Phys. Rev. Lett. 89 (2002) 050601.

[214] O. Mazonka, C. Jarzynski, Exactly solvable model illustrating far-fromequilibrium predictions, cond-mat/9912121 (1999).

[215] O. Narayan, A. Dhar, Reexamination of experimental tests of the fluctuation theorem, J. Phys. A 37 (2004) 63.

[216] M. Baiesi, T. Jacobs, C. Maes, N. S. Skantzos, Fluctuation symmetries for work, heat, Phys. Rev. E 74 (2006) 021111.

[217] A. Puglisi, L. Rondoni, A. Vulpiani, Relevance of initial and final conditions for the fluctuation relation in markov processes, J. Stat. Mech. (2006) P08010.

[218] R. J. Harris, A. Rákos, M. Schütz, Breakdown of Gallavotti-Cohen symmetry for stochastic dynamics, Europhys. Lett. 75 (2006) 227.

[219] Y. Oono, M. Paniconi, Steady state thermodynamics, Progr. Theor. Phys. Suppl. 130 (1998) 29.

[220] T. Hatano, S. Sasa, Steady-state thermodynamics of Langevin systems, Phys. Rev. Lett. 86 (2001) 3463.

[221] U. Seifert, Entropy production along a stochastic trajectory and an integral fluctuation theorem, Phys. Rev. Lett. 95 (2005) 040602.

[222] T. Schmiedl, T. Speck, U. Seifert, Entropy production for mechanically or chemically driven biomolecules, J. Stat. Phys. 128 (2006) 77.

[223] P. Visco, Work fluctations for a Brownian particle between two thermostats, J. Stat. Mech. (2006) P06006.

[224] A. Imparato, L. Peliti, Work-probability distribution in systems driven out of equilibrium, Phys. Rev. E 72 (2005) 046114. 
[225] A. Imparato, L. Peliti, G. Pesce, G. Rusciano, A. Sasso, Work and heat probability distribution of an optically driven brownian particle: Theory and experiments, arXiv:arXiv:0707.0439 (2007).

[226] A. Puglisi, P. Visco, E. Trizac, F. van Wijland, Dynamics of a tracer granular particle as a nonequilibrium markov process, Phys. Rev. E 73 (2006) 021301.

[227] J. L. Lebowitz, H. Spohn, A Gallavotti-Cohen-type symmetry in the large deviation functional for stochastic dynamics, J. Stat. Phys. 95 (1999) 333.

[228] P. Gaspard, Time-reversed dynamical entropy and irreversibility in markovian random processes, J. Stat. Phys. 117 (2004) 599.

[229] J. Farago, Injected power fluctuations in langevin equation, J. Stat. Phys. 107 (2002) 781.

[230] J. Farago, Power fluctuations in stochastic models of dissipative systems, Physica A 331 (2004) 69.

[231] R. van Zon, E. G. D. Cohen, Extension of the fluctuation theorem, Phys. Rev. Lett. 91 (2003) 110601.

[232] F. Bonetto, G. Gallavotti, P. L. Garrido, Chaotic principle: an experimental test, Physica D 105 (1997) 226.

[233] S. Lepri, L. Rondoni, G. Benettin, The Gallavotti-Cohen fluctuation theorem for a nonchaotic model, J. Stat. Phys. 99 (2000) 857.

[234] G. Benettin, L. Rondoni, A new model for the transport of particles in a thermostatted system, Math. Phys. Electronic J. 7 (2001) 3.

[235] L. Rondoni, G. P. Morriss, Large, fluctuations and axiom-C structures in deterministically thermostatted systems, Open Syst. Information Dynam. 10 (2003) 105.

[236] A. Giuliani, F. Zamponi, G. Gallavotti, Fluctuation relation beyond linear response theory, J. Stat. Phys. 119 (2005) 909.

[237] T. Gilbert, Fluctuation theorem applied to the Nosé-Hoover thermostated Lorentz gas, Phys. Rev. E 73 (2006) 035102.

[238] S. Lepri, R. Livi, A. Politi, Energy transport in anharmonic lattices close to, far from equilibrium, Physica D 119 (1998) 140.

[239] L. Rondoni, E. Segre, Fluctuations in two-dimensional reversibly damped turbulence, Nonlinearity 12 (1999) 1471.

[240] D. J. Searles, D. J. Evans, A fluctuation theorem for heat flow, Int. J. Thermophys. 22 (2001) 123.

[241] D. J. Evans, D. J. Searles, Fluctuation theorem for Hamiltonian systems: Le Chatelier's principle, Phys. Rev. E 63 (2001) 051105. 
[242] E. Fermi, J. Pasta, S. Ulam, Studies of nonlinear problems (Los Alamos Report LA-1940), reprinted in Segre E (ed) 1965 Collected papers of Enrico Fermi (Chicago: University of Chicago Press), 1955.

[243] P. Visco, A. Puglisi, A. Barrat, E. Trizac, F. van Wijland, Injected power and entropy flow in a heated granular gas, Europhys. Lett. 72 (2005) 55.

[244] P. Visco, A. Puglisi, A. Barrat, E. Trizac, F. van Wijland, Fluctuations of power injection in randomly driven granular gases, J. Stat. Phys. 125 (2006) 519 .

[245] C. Giardinà, J. Kurchan, L. Peliti, Direct evaluation of large-deviation functions, Phys. Rev. Lett. 96 (2006) 120603.

[246] S. Ciliberto, C. Laroche, An experimental test of the Gallavotti-Cohen fluctuation theorem, J. Phys. IV 8 (1998) 215.

[247] D. M. Carberry, J. C. Reid, G. M. Wang, E. M. Sevick, D. J. Searles, D. J. Evans, Fluctuations and irreversibility: an experimental demonstration of a second-law-like theorem using a colloidal particle held in an optical trap, Phys. Rev. Lett. 92 (2004) 140601.

[248] K. Feitosa, N. Menon, Fluidized granular medium as an instance of the fluctuation theorem, Phys. Rev. Lett. 92 (2004) 164301.

[249] N. Garnier, S. Ciliberto, Nonequilibrium fluctuations in a resistor, Phys. Rev. E 71 (2005) 060101.

[250] X. D. Shang, P. Tong, K. Q. Xia, Test od steady-state fluctuation theorem in turbulent Rayleigh-Bénard convection, Phys. Rev. E 72 (2005) 015301.

[251] F. Douarche, S. Joubaud, N. B. Garnier, A. Petrosyan, S. Ciliberto, Work fluctuation theorems for harmonic oscillators, Phys. Rev. Lett. 97 (2006) 140603.

[252] T. Tietz, S. Schuler, S. Speck, U. Seifert, J. Wrachtrup, Measurement of stochastic entropy production, Phys. Rev. Lett. 97 (2006) 050602.

[253] F. Zamponi, Is it possible to experimentally verify the fluctuation relation? a review of theoretical motivations and numerical evidence, J. Stat. Mech. (2007) P02008.

[254] F. Douarche, S. Ciliberto, A. Petrosyan, I. Rabbiosi, An experimental test of the Jarzynski equality in a mechanical experiment, Europhys. Lett. 70 (2005) 593.

[255] V. Blickle, T. Speck, L. Helden, U. Seifert, C. Bechinger, Thermodynamics of a colloidal particle in a time-dependent nonharmonic potential, Phys. Rev. Lett. 96 (2006) 070603.

[256] J. Liphardt, S. Dumont, S. B. Smith, I. Tinoco, C. Bustamante, Equilibrium information from nonequilibrium measurements in an experimental test of Jarzynski's equality, Science 296 (2002) 1832. 
[257] D. Collin, F. Ritort, C. Jarzynski, S. B. Smith, I. Tinoco, C. Bustamante, Verification of the Crooks fluctuation theorem, recovery of RNA folding free energies, Nature 437 (2005) 231.

[258] E. H. Trepagnier, C. Jarzynski, F. Ritort, G. E. Crooks, C. Bustamante, J. Liphardt, Experimental test of Hatano, Sasa's nonequilibrium steady-state equality, Proc. Nat. Acad. Sciences 101 (2004) 15038.

[259] F. Ritort, Work fluctuations, transient violations of the second law, free-energy recovery methods: Perspectives in theory, experiments, Poincaré Seminar 2 (2003) 195.

[260] A. Puglisi, P. Visco, A. Barrat, E. Trizac, F. van Wijland, Fluctuations of internal energy flow in a vibrated granular gas, Phys. Rev. Lett. 95 (2005) 110202.

[261] S. Aumaître, S. Fauve, S. McNamara, P. Poggi, Power injected in dissipative systems and the fluctuation theorem, Eur. Phys. J. B 19 (2000) 449.

[262] J. Hermans, Simple analysis of noise and hysteresis in (slow-growth) free energy simulations, J. Phys. Chem. 95 (1991) 9029.

[263] G. Hummer, A. Szabo, Free energy reconstructon from nonequilibrium singlemolecule pulling experiments, Proc. Natl. Acad. Sci. U.S.A. 98 (2001) 3658.

[264] D. A. Hendrix, C. Jarzynski, A "fast growth" method of computing free energy differences, J. Chem. Phys. 114 (2001) 5974.

[265] F. Ritort, Nonequilibrium fluctuations in small systems: From physics to biology, invited contribution to "Advances in Chemical Physics" (to appear) 137 (2007) xx.

[266] R. C. Lua, A. Y. Grosberg, Practical applicability of the Jarzynski relation in statistical mechanics: A pedagogical example, J. Phys. Chem. B 109 (2005) 6805 .

[267] L. Onsager, S. Machlup, Fluctuations and irreversible processes, Phys. Rev. 91 (1953) 1505.

[268] S. Machlup, L. Onsager, Fluctuations and irreversible process. II. Systems with kinetic energy, Phys. Rev. 91 (1953) 1512.

[269] H. Grabert, R. Graham, M. S. Green, Fluctuations and nonlinear irreversible processes. II, Phys. Rev. A 21 (1980) 2136.

[270] M. I. Freidlin, A. D. Wentzell, Random Perturbation of Dynamical Systems, Springer-Verlag, New York, 1998.

[271] G. L. Eyink, J. L. Lebowitz, H. Spohn, Hydrodynamics and fluctuations outside of local equilibrium: Driven diffusive systems, J. Stat. Phys. 83 (1996) 385 . 
[272] L. Bertini, A. D. Sole, D. Gabrielli, G. Jona-Lasinio, C. Landim, Macroscopic fluctuation theory for stationary non-equilibrium states, J. Stat. Phys. 107 (2002) 635 .

[273] B. Derrida, J. L. Lebowitz, E. R. Speer, Large deviation of the density profile in the steady state of the open symmetric simple exclusion process, J. Stat. Phys. 107 (2002) 599.

[274] L. A. Bunimovich, Y. G. Sinai, N. I. Chernov, Statistical properties of twodimensional hyperbolic billiards, Russian Math. Surveys 46 (1991) 47.

[275] A. Gamba, L. Rondoni, Current fluctuations in the nonequilibrium Lorentz gas, Physica A 340 (2004) 274.

[276] C. Giberti, L. Rondoni, C. Vernia, Asymmetric fluctuation-relaxation paths in FPU models, Physica A 365 (2006) 229.

[277] C. Paneni, D. J. Searles, L. Rondoni, Temporal asymmetry of fluctuations in nonequilibrium states, J. Chem. Phys. 124 (2006) 114109.

[278] C. Paneni, D. J. Searles, L. Rondoni, Temporal asymmetry of fluctuations in nonequilibrium steady states: Fluctuation paths, correlation functions and nonlinear response theory, submitted (2007).

[279] B. Derrida, J. L. Lebowitz, E. R. Speer, Exact free energy functional for a driven diffusive open stationary nonequilibrium system, Phys. Rev. Lett. 89 (2001) 030601.

[280] T. Pöschel, S. Luding (Eds.), Granular Gases, Springer, Berlin, 2001, Lecture Notes in Physics 564.

[281] T. Pöschel, N. Brilliantov (Eds.), Granular Gas Dynamics, Springer, Berlin, 2003, Lecture Notes in Physics 624.

[282] T. P. C. van Noije, M. H. Ernst, Velocity distributions in homogeneous granular fluids: the free and the heated case, Granular Matter 1 (1998) 57-64.

[283] A. Puglisi, F. Cecconi, A. Vulpiani, Models of fluidized granular materials: examples of non-equilibrium stationary states, J. Phys.: Condens. Matter 17 (S2715) 2005.

[284] V. Garzó, J. M. Montanero, Transport coefficients of a heated granular gas, Physica A 313 (2002) 336.

[285] G. A. Bird, Molecular Gas Dynamics and the Direct Simulation of Gas Flows, Clarendon, Oxford, 1994.

[286] J. M. Montanero, A. Santos, Computer simulation of uniformly heated granular fluids, Granular Matter 2 (2000) 53.

[287] J. M. Montanero, V. Garzò, Monte Carlo simulation of the homogeneous cooling state for a granular mixture, Granular Matter 4 (2002) 17. 
[288] R. S. Ellis, The theory of large deviations: from Boltzmann's 1877 calculation to equilibrium macrostates in 2d turbulence, Physica D 133 (1999) 106.

[289] S. R. S. Varadhan, Large deviations and entropy, in: A. Greven, G. Keller, D. Warnecke (Eds.), Entropy, Princeton University Press, 2003, p. 199.

[290] J. Guckenheimer, P. Holmes, Nonlinear oscillation, dynamical systems, and bifurcations of vector fields, Spriger-Verlag, Berlin, 1986.

[291] D. Ruelle, Elements of differentiable dynamics and bifurcation theory, Academic Press, London, 1989.

[292] W. Parry, Synchronisation of canonical measures for hyperbolic attractors, Comm. Math. Phys. 106 (1986) 267.

[293] J. Lloyd, M. Niemeyer, L. Rondoni, G. P. Morriss, The nonequilibrium Lorentz gas, Chaos 5 (1995) 536.

[294] P. Gaspard, Chaos, scattering and statistical mechanics, Cambridge University Press, 1998.

[295] G. P. Morriss, L. Rondoni, Periodic orbit expansions for the Lorentz gas, J. Stat. Phys. 75 (1994) 553.

[296] D. Ruelle, Smooth dynamics, new theoretical ideas in nonequilibrium statistical mechanics, J. Stat. Phys. 95 (1999) 393.

[297] R. D. Astumian, The unreasonable effectiveness of equilibrium theory for interpreting nonequilibrium experiments, Am. J. Phys 74 (2006) 683. 